\title{
Mathematical Analysis and Simulation of Field Models in Accelerator Circuits
}

Mathematische Analyse und Simulation von Feldmodellen in Beschleunigerschaltungen Zur Erlangung des akademischen Grades Doktor-Ingenieur (Dr.-Ing.) Genehmigte Dissertation von Idoia Cortes Garcia aus Sant Celoni, Spanien Fachbereich Elektrotechnik und Informationstechnik, Technische Universität Darmstadt Tag der Einreichung: 13.03.2020, Tag der Prüfung: 28.04.2020

1. Gutachten: Prof. Dr. Sebastian Schöps

2. Gutachten: Prof. Dr. Caren Tischendorf Darmstadt, 2020
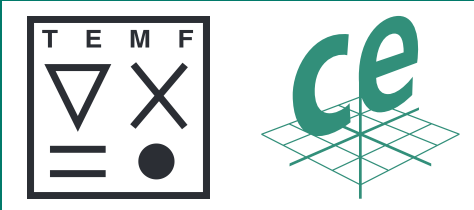
Mathematical Analysis and Simulation of Field Models in Accelerator Circuits Mathematische Analyse und Simulation von Feldmodellen in Beschleunigerschaltungen

Genehmigte Dissertation von Idoia Cortes Garcia aus Sant Celoni, Spanien

Fachbereich Elektrotechnik und Informationstechnik, Technische Universität Darmstadt

1. Gutachten: Prof. Dr. Sebastian Schöps

2. Gutachten: Prof. Dr. Caren Tischendorf

Tag der Einreichung: 13.03.2020

Tag der Prüfung: 28.04.2020

Darmstadt, 2020

Bitte zitieren Sie dieses Dokument als:

URN: urn:nbn:de:tuda-tuprints-117508

URL: http://tuprints.ulb.tu-darmstadt.de/id/eprint/11750

Dieses Dokument wird bereitgestellt von tuprints,

E-Publishing-Service der TU Darmstadt

http://tuprints.ulb.tu-darmstadt.de

tuprints@ulb.tu-darmstadt.de

Nutzungsrechte gemäß UrhG 


\section{Erklärungen laut Promotionsordnung}

\section{$\S 8$ Abs. 1 lit. c Promo}

Ich versichere hiermit, dass die elektronische Version meiner Dissertation mit der schriftlichen Version übereinstimmt.

\section{§8 Abs. 1 lit. d Promo}

Ich versichere hiermit, dass zu einem vorherigen Zeitpunkt noch keine Promotion versucht wurde. In diesem Fall sind nähere Angaben über Zeitpunkt, Hochschule, Dissertationsthema und Ergebnis dieses Versuchs mitzuteilen.

\section{$\S 9$ Abs. 1 Promo}

Ich versichere hiermit, dass die vorliegende Dissertation selbstständig und nur unter Verwendung der angegebenen Quellen verfasst wurde.

\section{§9 Abs. 2 Promo}

Die Arbeit hat bisher noch nicht zu Prüfungszwecken gedient.

Darmstadt, 13.03.2020

I. Cortes Garcia 
"Who would have guessed reading and writing would pay off?"

Homer Simpson 


\section{Zusammenfassung}

Bei der Simulation des physikalischen Verhaltens von Bauelementen und der sie umgebenden Schaltungstechnik wird üblicherweise ein Netzwerkmodellierungsansatz gewählt, bei dem die Geräte durch SpannungsStrom-Beziehungen beschrieben werden. Für einige Anwendungen liefert diese Vereinfachung jedoch nicht die erforderliche Genauigkeit. In diesen Fällen kann eine verfeinerte Modellierung durchgeführt werden, bei der eine räumlich-verteilte partielle Differentialgleichung die erforderlichen physikalischen Größen modelliert und an die klassischen Schaltungsgleichungen gekoppelt wird. Das resultierende Gleichungssystem weist oft ein multiskalen, multiraten und sogar multiphysikalisches Verhalten auf. Um dies effizient zu simulieren, werden involvierte Algorithmen verwendet. Daher ist die Strukturanalyse des Systems fundamental, um es numerisch korrekt zu behandeln und sicherzustellen, dass die Algorithmen wie erwartet konvergieren. Diese Arbeit befasst sich mit der mathematischen Analyse dieser Systeme, sowie deren Simulation.

Typischerweise sind die Gleichungssysteme, die aus Schaltungen mit semidiskreten verfeinerten Modellen entstehen, differential-algebraische Gleichungen. Die potentiellen numerischen und analytischen Schwierigkeiten, die bei ihnen auftreten, können im Kontext ihres Index' untersucht werden. In diesem Rahmen werden drei verallgemeinerte Schaltungselemente definiert, die eine Klassifizierung der verfeinerten Modelle ermöglichen. Somit kann der Index des gesamten gekoppelten Systems ausschließlich durch topologische Eigenschaften des Netwerks ermittelt werden. Verschiedene Näherungen der Maxwell-Gleichungen werden durch die verallgemeinerten Elementdefinitionen klassifiziert, um somit die Index-Eigenschaften des gekoppelten Systems zu bestimmen.

Zwei Algorithmen werden für die Simulationen untersucht. Das Waveform-Relaxationsverfahren wird zunächst für die Kosimulation von Feld un Netzwerk analysiert, die aus magnetoquasistatischen Feldern mit Wirbelstromeffekten auf supraleitenden Spulen entstehen. Die Konvergenz des Waveform-Relaxationsverfahrens wird mittels Optimised-Schwarz-Methoden beschleunigt. Hiermit wird der Informationsaustausch zwischen beiden Subsystemen verbessert. Für die weitere Beschleunigung der Simulationszeit, wird das zeitparallele Verfahren Parareal analysiert. Hierfür wird zuerst der Algorithmus im Kontext der Differential-algebraischen Gleichungen untersucht, wobei seine Anwendbarkeit auf nichtlineare Systeme höheren Index, die z.B. aus den Schaltungsgleichungen entstehen, ermittelt wird. Anschließend werden zwei Ansätze für die Kombination von Parareal und Waveform-Relaxation untersucht. Einer der Ansätze ist speziell für Feld-Netzwerk gekoppelte Systeme entworfen und ergibt einen mikro-makro-ähnlichen Parareal-Algorithmus. Der Ansatz kann jedoch auch auf andere Arten gekoppelter Systeme angewendet werden.

Zuletzt werden numerische Tests an Feld-Netzwerk gekoppelten Systemen durchgeführt, um die theoretischen Resultate sowie die Effizienz der vorgeschlagenen Algorithmen nachzuprüfen. 



\section{Abstract}

Typically in electrical engineering a network modelling approach for the simulation of devices and their surrounding circuitry is taken, where each device is considered by a voltage-to-current relation. For some applications, however, this simplification does not yield the required accuracy. In these cases, refined modelling can be performed, where a spatially distributed partial differential equation modelling the required physical quantity is coupled to the classic network equations. The resulting coupled system of equations often exhibits a multiscale, multirate and even multiphysical behaviour that is tackled with involved algorithms so as to efficiently simulate it. Its structural analysis is therefore important, to numerically treat the system appropriately and to ensure that the algorithms converge properly. This thesis deals with the mathematical analysis of these type of systems as well as their simulation.

The systems of equations obtained from circuits with semidiscrete refined models are typically differential algebraic equations. Their numerical and analytical difficulties is studied in the context of their differential algebraic index. For that, three generalised circuit element definitions are given, that allow the classification of the refined models. Hereby, the index of the entire coupled system can be specified by means of topological properties of the circuit. Several approximations to Maxwell's equations are classified with the generalised element definitions to obtain the index properties of the field-circuit coupled systems.

For the simulation two algorithms are studied. First the co-simulation waveform relaxation method is analysed for field-circuit coupled systems arising from magnetoquasistatic fields with eddy current effects on superconducting coils. The convergence of the algorithm is sped up by means of optimised Schwarz methodologies. Here, the information exchange between both subsystems is improved by a linear combination of the coupling conditions. To further speed up simulation time, the parallel-in-time method Parareal is analysed. The algorithm is investigated in the context of differential algebraic equations by studying its applicability to nonlinear higher index systems arising e.g. from circuit simulation. Finally, two approaches are proposed for the combination of Parareal and waveform relaxation. One of them is specifically designed for field-circuit coupled systems and yields a micro-macro-like Parareal algorithm. However, the idea behind it can be applied to other type of coupled systems.

Numerical tests of field-circuit coupled systems are made to underline the results obtained from the mathematical theory as well as test the efficiency of the proposed algorithms. 



\section{Contents}

List of figures $\quad$ xiii

1 Introduction 1

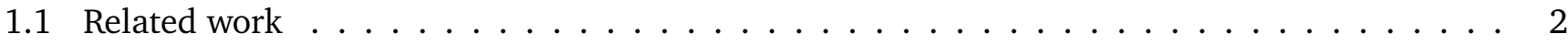

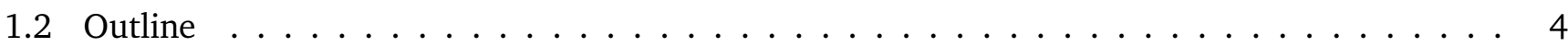

2 Modelling 6

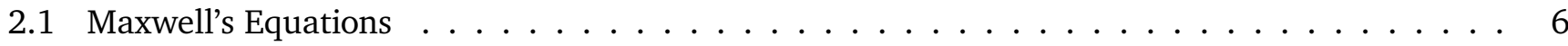

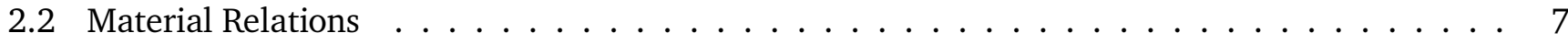

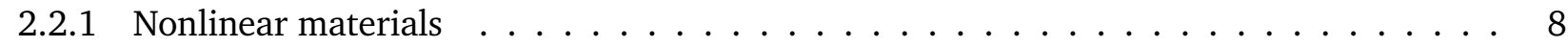

2.3 Boundary and Initial Conditions . . . . . . . . . . . . . . . . . . . 9

2.4 Static and Quasistatic Fields . . . . . . . . . . . . . . . . . . . 10

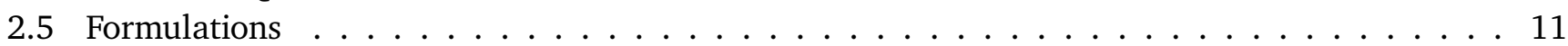

2.5 .1 Full Maxwell . . . . . . . . . . . . . . . . . . . . . 11

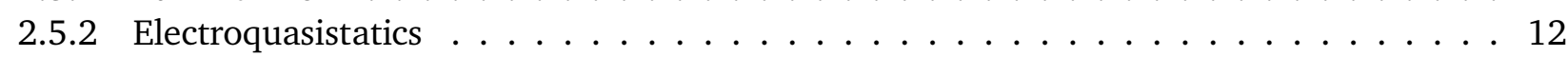

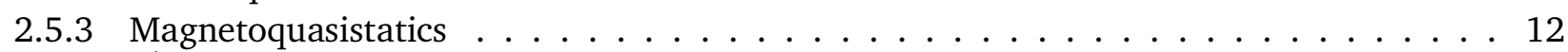

$2.5 .4 \vec{A}-\phi$ formulation $\ldots \ldots \ldots \ldots \ldots \ldots \ldots \ldots$

$2.5 .5 \vec{T}-\Omega$ formulation $\ldots \ldots \ldots \ldots \ldots \ldots \ldots \ldots \ldots$

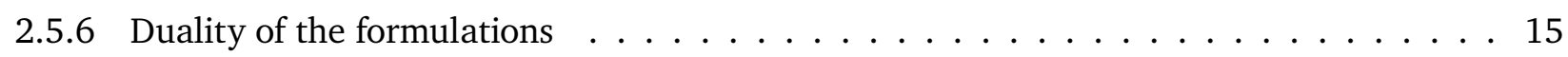

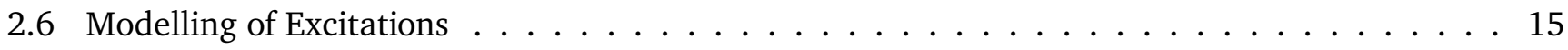

2.6.1 Excitation with winding density functions $\ldots \ldots \ldots \ldots \ldots$

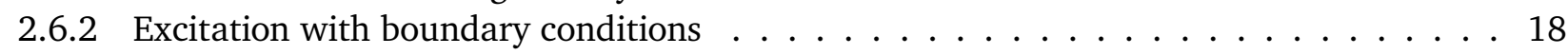

2.7 Modelling of Superconducting Magnets . . . . . . . . . . . . . . . . . . . 20

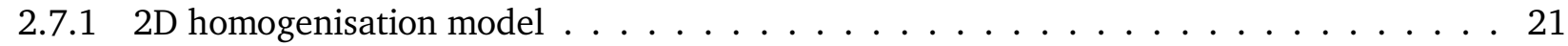

2.7 .2 Heat Equation . . . . . . . . . . . . . . . . . . . . 21

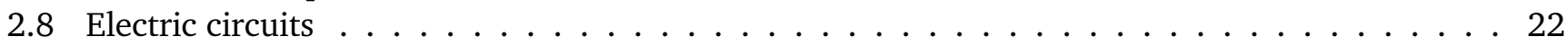

2.8.1 From Maxwell to circuits . . . . . . . . . . . . . . . . . . . . . . . 22

2.8 .2 Lumped element models . . . . . . . . . . . . . . . . . . . . . . . 24

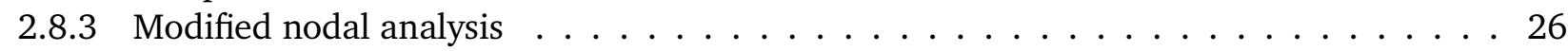

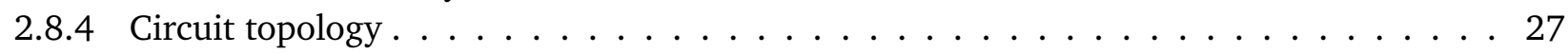

3 Numerical Methods and Model Analysis $\quad 29$

3.1 Space Discretisation . . . . . . . . . . . . . . . . . . . . . . . . 29

3.1 .1 Finite integration technique . . . . . . . . . . . . . . . 29

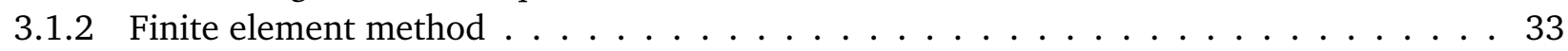

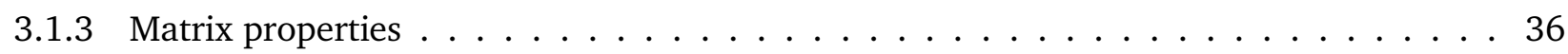

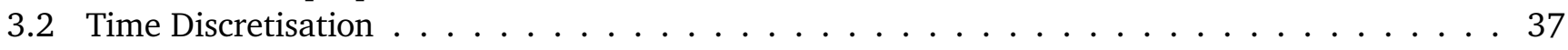

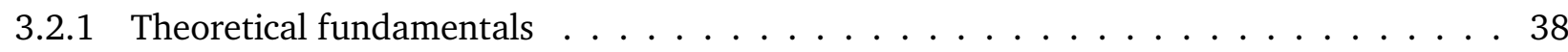




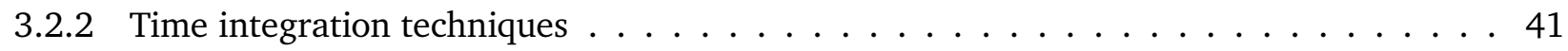

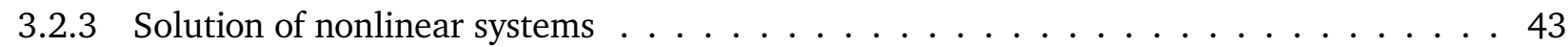

3.3 Differential Algebraic Equations . . . . . . . . . . . . . . . . . . . . 44

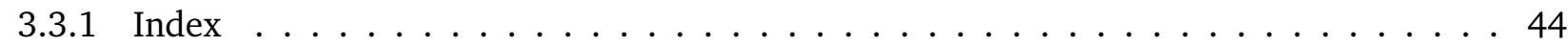

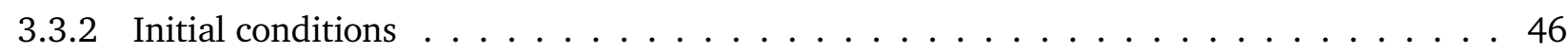

3.3 .3 Numerical methods for DAEs . . . . . . . . . . . . . . . . . 47

4 Structural Analysis of the Coupled Systems 49

4.1 Generalised Circuit Elements _ . . . . . . . . . . . . . . . . . . . . . 50

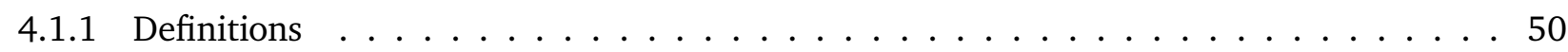

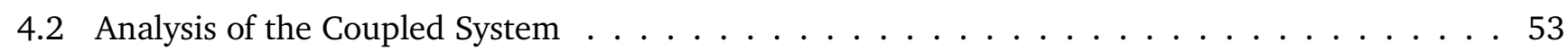

4.2 .1 Generalised elements in MNA . . . . . . . . . . . . . . . . . 54

4.2 .2 DAE index of the circuit $\ldots \ldots \ldots \ldots \ldots \ldots \ldots$

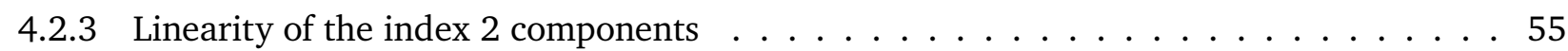

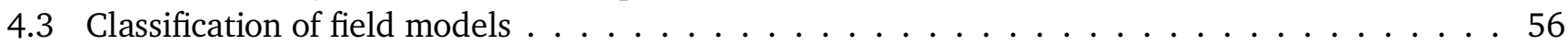

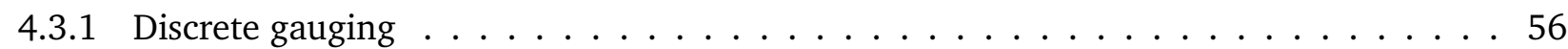

4.3 .2 Inductance-like elements . . . . . . . . . . . . . . . . . . . 57

4.3 .3 Capacitance-like element . . . . . . . . . . . . . . . . . . 64

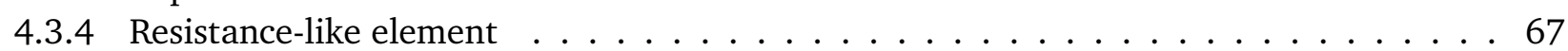

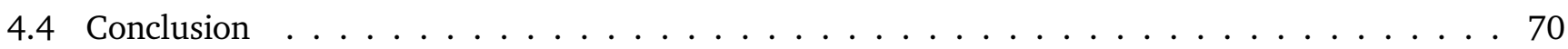

5 Iterative Methods in Time Domain $\quad 71$

5.1 Optimised Waveform Relaxation . . . . . . . . . . . . . . . . . . 71

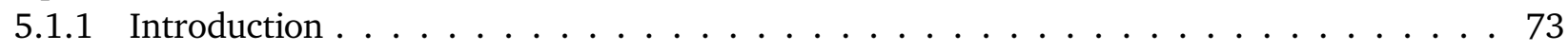

5.1 .2 Optimised Schwarz for accelerator magnets $\ldots \ldots \ldots \ldots$

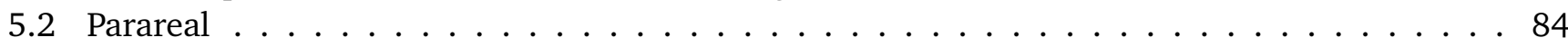

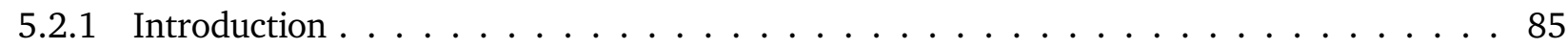

5.2 .2 Parareal for differential algebraic equations $\ldots \ldots \ldots \ldots$

5.3 Parallelised Waveform Relaxation . . . . . . . . . . . . . . . . . . . . . 93

5.3.1 Waveform relaxation and Parareal . . . . . . . . . . . . . . . . . 94

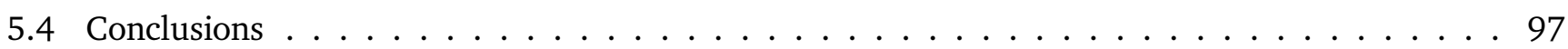

6 Numerical Examples $\quad 99$

6.1 DAE Index of Refined Models . . . . . . . . . . . . . . . . . . . . . . 999

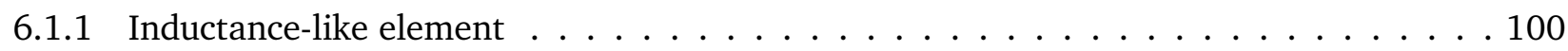

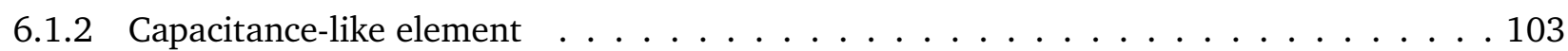

6.1 .3 Conclusions . . . . . . . . . . . . . . . . . . . . . 104

6.2 Waveform relaxation for index 2 circuit $\ldots \ldots \ldots$. . . . . . . . . . . . 105

6.3 Optimised Co-Simulation of Field-Circuit Systems ～. . . . . . . . . . . . . . . . 109

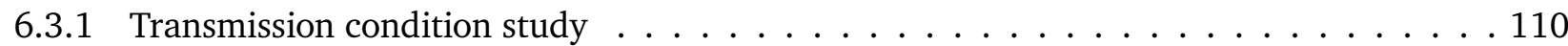

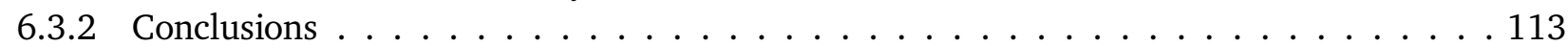

6.4 Parareal for DAEs with Implicit Euler . . . . . . . . . . . . . . . . . . . . . . . 113

6.5 Parallelised Co-Simulation of Field-Circuit Systems ～. . . . . . . . . . . . . 116

6.5 .1 Parallelised waveform relaxation $\ldots \ldots \ldots \ldots \ldots \ldots \ldots \ldots$

6.5 .2 Field-circuit parallelised waveform relaxation $\ldots \ldots \ldots$. . . . . . . . . . . . . . . . .

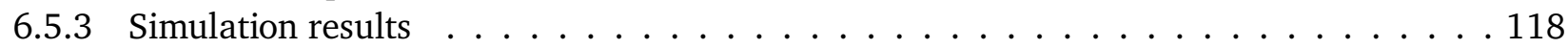

6.5 .4 Conclusion . . . . . . . . . . . . . . . . . . . . . 119 
7 Summary and Outlook

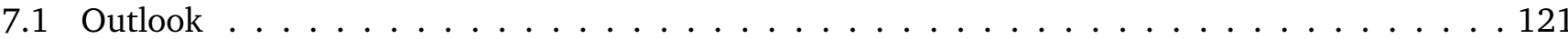

A Appendix 



\section{List of figures}

1.1 Sketch of refined modelling in field-circuit coupling. . . . . . . . . . . . . . . . . 1

1.2 Cross section of superconducting quadrupole magnet. . . . . . . . . . . . . . . 2

2.1 Sketch of the domain $\Omega \ldots \ldots \ldots \ldots \ldots \ldots \ldots \ldots \ldots$

2.2 Sketch of the partition of the boundary $\Gamma=\Gamma_{\text {dir }, 0} \cup \Gamma_{\text {neu, } 0} \ldots \ldots \ldots \ldots \ldots$

2.3 Maxwell house based on [64] . . . . . . . . . . . . . . . . . . . . . . 15

2.4 Sketch of stranded conductor cable's cross-section. . . . . . . . . . . . . . . . . . . 16

2.5 Maxwell house's extension with winding density functions [55] . . . . . . . . . . . . . 18

2.6 Sketch of the partition of the boundary $\Gamma \ldots \ldots \ldots \ldots \ldots$

2.7 Sketch of Rutherford cable's cross-section. . . . . . . . . . . . . . . . . . . . . 20

2.8 Sketch of the volume around circuit node and area inside circuit loop. . . . . . . . . . . 23

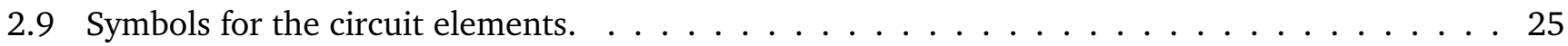

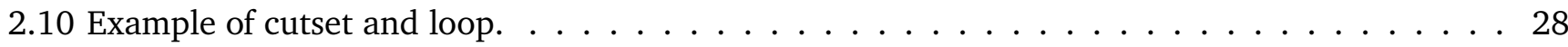

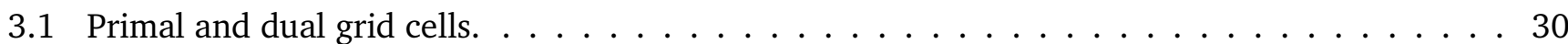

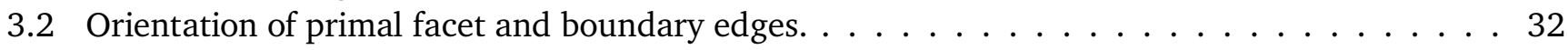

3.3 Sketch of dual facet with its normal vector and surrounding primal volumes. . . . . . . . . . 32

3.4 Sketch of local error propagation in the global error. . . . . . . . . . . . . . . . . 39

3.5 Stability region of explicit Euler and implicit Euler. . . . . . . . . . . . . . . . . . 41

3.6 Stability regions for an A-stable method and an $\mathrm{A}(\alpha)$-stable method. $\ldots \ldots \ldots$. . . . . . 42

5.1 Multiscale and multirate behaviour of LHC's quench simulation problem. . . . . . . . . . . 72

5.2 Schematic of waveform relaxation algorithm with two subsystems and windowing. . . . . . 73

5.3 Sketch of waveform relaxation (WR) scheme as heterogeneous domain decomposition. . . . . 76

5.4 Circuit stencil of superconducting magnet and heat equation. . . . . . . . . . . . . . 77

5.5 Sketch of Parareal jumps across windows. . . . . . . . . . . . . . . . . 85

5.6 Sketch of coupled Parareal and waveform relaxation. . . . . . . . . . . . . . . . 95

5.7 Sketch of fine and coarse models for Parareal applied to a field-circuit coupled problem example. 97

6.1 Inductance-like element example's geometry. . . . . . . . . . . . . . . . . . . . . 100

6.2 The considered index 1 and index 2 circuit examples. . . . . . . . . . . . . . . . . . 101

6.3 Simulation results for index 1 and index 2 perturbed and non perturbed circuits. . . . . . . . 102

6.4 Sketch of cable termination model. . . . . . . . . . . . . . . . . . . . . . . . . . 104

6.5 The considered index 1 and index 2 circuit examples. . . . . . . . . . . . . . . . 105

6.6 Electroquasistatic simulation results for index 1 and index 2 perturbed and non perturbed

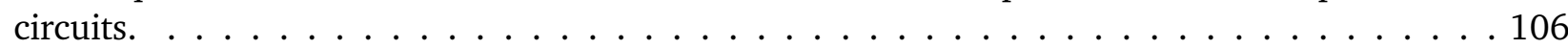

6.7 Index 2 circuits coupled to magnetoquasistatic field model with ground node potential $e_{0}=0.107$

6.8 Single phase isolation transformer in FEMM (see [162]). . . . . . . . . . . . . . . . 108

6.9 Voltage across transformer for monolithic solution "mon" and $k$-th WR iteration. . . . . . . . 109 
6.10 Superconducting magnet and protection circuit. . . . . . . . . . . . . . 110

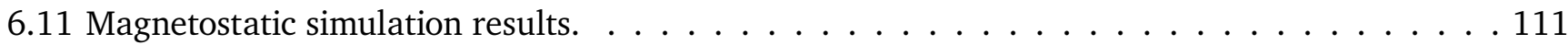

6.12 Correction term $\Delta v_{\mathrm{m}}$ between circuit and field solutions for eddy current simulation. . . . . . 112

6.13 Waveform relaxation iteration numbers in the first three windows of the eddy current simulation.112

6.14 Index 2 circuits with LI-cutsets. . . . . . . . . . . . . . . . . . . . . . 113

6.15 First circuit (Figure 6.14a) simulation results at the $k$-th Parareal iteration. . . . . . . . . 114

6.16 Second circuit (Figure 6.14b) simulation results at the $k$-th Parareal iteration. . . . . . . . . 115

6.17 Single-phase isolation transformer of model example in FEMM and surrounding rectifier circuit.116

6.18 Field-Circuit parallelised waveform relaxation coarse system model. . . . . . . . . . . . . 118

6.19 Effective number of linear system solves for field-circuit coupled systems with lamination model.119 


\section{Introduction}

Electromagnetic fields are present in industrial applications as well as everyday life. For example, electric machines have a broad variety of usage from electric power transmission in form of transformers to electric mobility as motors. Their prediction is of high importance for the design, optimisation and understanding, so as to ensure a proper functioning.

In electrical engineering, especially in electronics, often circuits are used to model the electric behaviour of the devices that are simulated and their interconnections. Here, the circuits are represented as graphs whose branches contain lumped element models that represent the devices. These type of models approximate the spatially distributed electromagnetic behaviour of the elements by algebraic or differential relations between currents through and voltages across the circuit's branches. In some applications, however, this simplification does not deliver the required information, e.g. for geometry optimisation or design. It is not able to capture all the required effects, such as e.g. spatially distributed eddy currents. One way to circumvent this is by means of refined modelling [1] (see Figure 1.1). This strategy involves coupling systems of partial differential equations describing spatially resolved physical phenomena to the network's system of equations. In this way, a detailed spatial information of the physical behaviour of important devices is obtained and the surround-

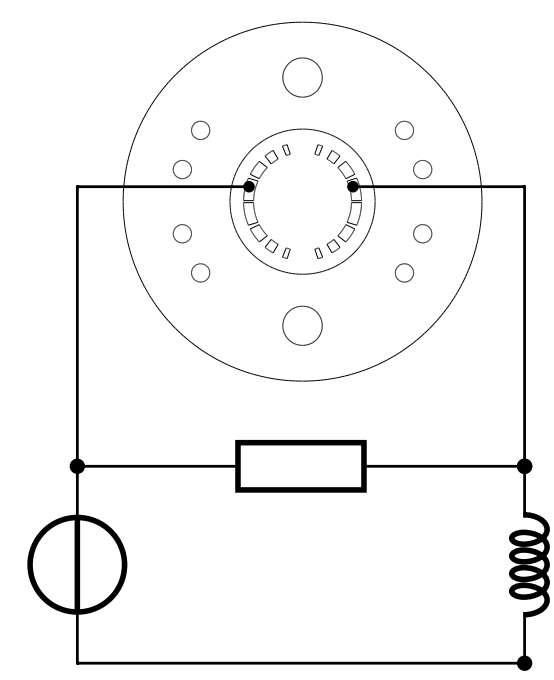

Figure 1.1: Sketch of refined modelling in field-circuit coupling. ing circuitry and interconnections between different elements can still be described by means of simple electric network equations. For the special case of electromagnetic fields described by Maxwell's equations coupled to circuits, the terminology "field-circuit coupling" is used [2], [3].

Even though the theory and methodologies used in this thesis can be employed for a large variety of fieldcircuit application examples, one main setting is motivating large part of the work; the simulation of the quench protection system of the LHC (Large Hadron Collider) at CERN [5]. Here, thousands of superconducting magnets are used to produce high magnetic fields. These apply an electromagnetic force to the beam of particles travelling through the collider, hereby bending its trajectory or focusing it (see Figure 1.2). The so-called critical surface of a superconducting material depends on its temperature, magnetic flux density and current density. Once this critical surface is crossed, the material quenches, that is, it loses its superconductivity. Therefore, to guarantee the material remains in a superconducting state, it has to be kept at very low temperatures $(1.9 \mathrm{~K})$. Nevertheless, a quench can happen e.g. due to beam losses, mechanical movement or cryogenic malfunction. In that case, ohmic losses due to the large magnetic energy stored in the magnet may heat it up, which can highly damage it as well as its surrounding circuitry. Therefore, the magnets are 

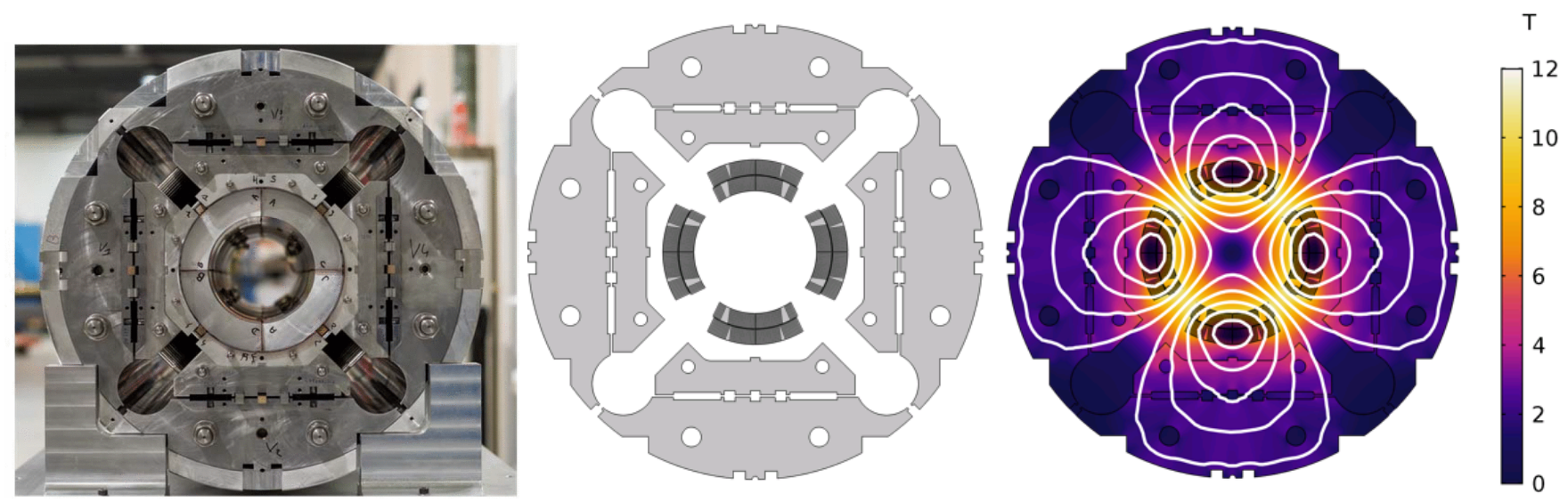

Figure 1.2: Cross section of superconducting quadrupole magnet. Picture of dummy magnet with aluminium coils (left). Figure taken from [4]. (c) [2019] IEEE. Magnetic flux density simulation results (right). Figure taken from [5]. (C) [2017] IEEE.

connected to a surrounding protection system [6], [7]. To evaluate worst case scenarios, the simulation of quench propagation and protection measures is of high importance. The proper description of the mutual influence between the protection systems, such as the quench heaters [6] or coupling-loss induced quench system [7], the magnet and the surrounding circuitry requires refined modelling. Therefore, field-circuit coupling is performed. The highly multiphysical properties of the underlying application involves also coupling other systems of equations that are important for the quench simulation, such as heat propagation [8] or even mechanical models [9].

To this end, the STEAM (simulation of transient effects in accelerator magnets) project has been formed [5]. The aim of the project is to tackle the multiphysical, multirate and multiscale problem arising from quench simulation by means of state-of-the-art techniques. Hereby, a framework and hierarchical co-simulation tools are established, which allow for coupling all the different physical phenomena involved in quench propagation and simulating their mutual influence.

To ensure the proper function of the complex algorithms that are required to simulate such sophisticated coupled systems, a proper mathematical understanding of the systems that are involved as well as the techniques that are used is important. Therefore, this thesis focuses on two main aspects; first, the structural analysis of the systems of equations obtained from refined modelling, and second the study of the iterative time domain methods that are used to simulate these type of systems.

\subsection{Related work}

The strategy of refined modelling inside networks is a well established technique to describe special components in e.g. circuits. Already in 1976, the idea of coupling an external electric network to a finite element field model arose (see [10]). In [11], the magnetoquasistatic finite element model was coupled to a circuit described with loop analysis. Later works, such as [12], [13], introduced the terminology 'mixed-mode' modelling to denote the coupling of partial differential equation models of semiconductors with circuits and started using nodal analysis to describe the surrounding electric network. Finally, in e.g. [14]-[16], the 
modified nodal analysis circuit equations were coupled to partial differential equations such as the transport equations for semiconductor, telegrapher's equations for transmission lines or magnetostatic Maxwell's equations for electric motors. The resulting systems of equations, after spatial discretisation, are typically of differential algebraic type.

The structural analysis of classic electric networks described with modified nodal analysis in terms of their differential algebraic system's properties is well known (see [17], [18]). To study their characteristics, the notion of index is used and the results are given in terms of topological features of the underlying network. Other works, such as [19], [20] performed the same structural analysis for the semidiscrete system of differential algebraic equations obtained from different field models. In refined modelling, the analysis is to be carried out for the entire coupled system of circuit equations and semidiscrete partial differential equations. This has been performed e.g. for models describing transmission lines, semiconductors or magnetic fields within circuits in e.g. [15], [21]-[23]. A more detailed literature review on the structural analysis of differential algebraic equations obtained from circuits coupled to semidiscrete partial differential equations can be found in [24].

To efficiently solve the obtained ordinary differential or differential algebraic equations, iterative time domain methods can be employed. In this work, two methods are studied, the waveform relaxation technique and Parareal.

Waveform relaxation is a co-simulation algorithm presented in 1982 (see [25]), originally applied to the simulation of large networks. Classic convergence theory for these type of methods was carried out for ordinary differential equations. A detailed overview of iterative time domain methods, including the waveform relaxation technique, their mathematical analysis as well as their history for ordinary differential equations can be found in [26]. Later, in [27], [28] the convergence analysis was extended to specific structured systems of differential algebraic equations. In [29], [30] a more detailed convergence order analysis was given in terms of the coupling variables of the subsystems. Co-simulation techniques can often be efficiently applied to heterogeneously coupled systems such as the ones arising from field-circuit coupling. Among other methods [31], [32], waveform relaxation has also been used and studied for these specific type of subsystems [3], [33]. To speed up the convergence ot the waveform relaxation algorithm, optimised Schwarz method techniques can be employed. These type of algorithms were originally used as domain decomposition methods for time dependent partial differential equations [34], [35]. For a detailed overview on optimised Schwarz methods and their history see [36], [37]. Later, the same techniques were also applied to systems arising from electric networks [38], [39] or even to field-circuit coupled systems [40].

To speed up simulation time, parallelisation methods are nowadays in focus due to the high capability of modern computers to execute processes simultaneously. In addition to domain decomposition methods, that perform a spatial parallelisation, parallel-in-time methods can also be considered. Already in 1964, a parallelisation of ordinary differential equations was proposed in [41]. Years later, in 2001, the parallelin-time method Parareal was proposed in [42]. The convergence theory for the algorithm and its connection to multiple shooting methods was given later in [43]-[45]. However, the theory was only investigated in the context of ordinary differential equations, in contrast to multiple shooting methods, where an extension to differential algebraic equations was given e.g. in [46], [47]. Nevertheless, the algorithm was also applied to systems of (simple) differential algebraic equations in [48], [49]. The latter one briefly studied the algorithm in the context of the differential algebraic equations obtained from the simulation of an electric machine. Parareal has recently gained interest in the context of the simulation of electric motors (see e.g. [50], [51]).

Combining both iterative methods (Parareal and waveform relaxation) was already performed e.g. in [48], [52], [53]. A detailed survey on the history of parallel-in-time methods, including (Schwarz) waveform 
relaxation and Parareal can be found in [54].

\subsection{Outline}

The work is structured as follows. Chapter 2 introduces the theoretical fundamentals and systems of equations that model the physical phenomena simulated and analysed in this thesis. Here, the description of electromagnetic fields with Maxwell's equations and their different approximations is presented. For the simulation of quench protection and propagation, homogenisation models are used to describe the eddy current effects on superconducting accelerator magnets. The equations to describe the magnetothermal phenomena for this particular application are given. Finally, the system of equations that is obtained to describe electric networks with modified nodal analysis is derived.

In Chapter 3, the classic numerical methods that are used to simulate systems of partial differential equations (PDEs) and differential algebraic equations (DAEs) are presented. The first part deals with space discretisation methodologies and the second part introduces classic time integration theory and algorithms. Finally, the special characteristics of DAEs are discussed. Here, the numerical and analytical difficulties they can pose are explained and the time integration techniques are extended to these type of systems.

After the first two chapters have introduced the necessary theoretical fundamentals for the thesis, the following parts present original results and thus constitute the core of this work.

Chapter 4 deals with the structural analysis of the system of DAEs that is obtained when coupling refined models in circuits. Three generalised circuit elements are defined for the classification of refined models. These elements are combined with the circuit's system to obtained a large, coupled system of differential algebraic equations. Its index is given in terms of topological properties of the network. In the end, different approximations and formulations of Maxwell's equations are classified. The definitions, index theorem and classification results were presented in the publications [24], [55].

In Chapter 5 two iterative time domain methods are presented. Their study and analysis is motivated and performed for field-circuit coupled systems. However, the ideas and methodologies can also be applied to other types of systems. First, waveform relaxation is analysed as a co-simulation algorithm. Its convergence speed is studied and improved by means of optimised Schwarz methods, where an optimised transmission condition is derived for the information exchange between the field and circuit subsystems. The theoretical results of this section were published in [56]. The second method that is examined is Parareal. The algorithm is analysed and expanded to the context of DAEs. Finally, both waveform relaxation as well as Parareal are combined into one algorithm. Two different approaches are presented, the latter one to be applied to fieldcircuit coupled systems and yielding a micro-macro like Parareal algorithm. These two proposed algorithms were published in [57].

In Chapter 6, numerical examples are demonstrated, to underline the theoretical results of the previous chapters as well as test the efficiency of the proposed algorithms. First, a magnetoquasistatic and an electroquasistatic refined model are coupled to two circuits to test the DAE index results of the previous chapter. The simulations are carried out with Octave, the spatial discretisation with the in-house code NIOBE and the circuits are simulated with OCS (Octave Circuit Simulator). For the test of the optimised waveform relaxation algorithm of Section 5.1, the setting of a dipole accelerator magnet coupled to a surrounding circuit is used. The simulation results were obtained at CERN [56] with the in-house coupling tool STEAM ${ }^{1}$. To benchmark

${ }^{1}$ https://espace.cern.ch/steam 
the application of Parareal to DAEs, the algorithm is applied to two nonlinear circuit models that are simulated with OCS. Finally, the combination of Parareal and Waveform relaxation is used on a transformer model of FEMM (Finite Element Method Magnetics) with a surrounding circuit described by OCS. Two combinations are tested and evaluated.

The thesis ends with a summary and outlook in Chapter 7. 


\section{Modelling}

The different physical phenomena that are relevant through this work are described by either space and time dependent PDEs or only time dependent DAEs. This section presents the different systems of equations that are required later on, as well as the quantities that are involved. It introduces the different PDEs that are relevant for the description of electromagnetic fields, i.e. Maxwell's equations, and the simulation of the heat propagation, both relevant for the simulation of quench propagation and protection. Also, classical types of boundary conditions are presented. In the end, the basic concepts of circuit simulation are exposed and the system of DAEs that is used for their description is shown. The majority of its content and structure is follows [58] and [55].

\subsection{Maxwell's Equations}

Macroscopic electromagnetic fields are described by Maxwell's equations [59]-[61]. Those can be written in integral form for a system at rest and for all piecewise smooth compact oriented areas $A \subset \mathbb{R}^{2}$ and volumes $V \subset \mathbb{R}^{3}[62]$ as

$$
\begin{aligned}
\int_{\partial A} \vec{E} \cdot \mathrm{d} \vec{s} & =-\int_{A} \frac{\partial}{\partial t} \vec{B} \cdot \mathrm{d} \vec{A}, \\
\int_{\partial V} \vec{D} \cdot \mathrm{d} \vec{A} & =\int_{V} \rho \mathrm{d} V, \\
\int_{\partial A} \vec{H} \cdot \mathrm{d} \vec{s} & =\int_{A}\left(\frac{\partial}{\partial t} \vec{D}+\vec{J}\right) \cdot \mathrm{d} \vec{A}, \\
\int_{\partial V} \vec{B} \cdot \mathrm{d} \vec{A} & =0,
\end{aligned}
$$

where the electric field strength $\vec{E}$, the magnetic flux density $\vec{B}$, the magnetic field strength $\vec{H}$, the electric flux density $\vec{D}$ and the electric current density $\vec{J}$ are vector fields $\mathbb{R} \times \mathbb{R}^{3} \rightarrow \mathbb{R}^{3}$ depending on time $t \in \mathbb{R}$ and space $\vec{r} \in \mathbb{R}^{3}$. The electric charge density $\rho: \mathbb{R} \times \mathbb{R}^{3} \rightarrow \mathbb{R}$ is a scalar field. By using Stokes and Gauß's theorems, the following PDEs can be derived from (2.1), see e.g. [63, Chapter 1.1.2] for a mathematical 
discussion on their equivalence,

$$
\begin{aligned}
\operatorname{curl} \vec{E} & =-\frac{\partial}{\partial t} \vec{B}, \\
\operatorname{curl} \vec{H} & =\frac{\partial}{\partial t} \vec{D}+\vec{J}, \\
\operatorname{div} \vec{D} & =\rho, \\
\operatorname{div} \vec{B} & =0 .
\end{aligned}
$$

In the general case, Maxwell's equations are considered in the whole domain $\mathbb{R}^{3}$ and time $\mathbb{R}$. However, in order to focus only on the relevant parts, the solution is reduced to finite domains $\Omega \subset \mathbb{R}^{3}$ and $\mathcal{I} \subset \mathbb{R}$ with appropriate boundary conditions (BCs) and initial conditions (ICs), respectively.

Assumption 1 (Domain). We consider the domain $\Omega \subset \mathbb{R}^{3}$ to be open, bounded, Lipschitz and contractible, that is, simply connected with connected boundary (see e.g., [64]).

The assumption ensures that the so-called de Rham's cohomology [64], which describes the relation between the electromagnetic fields in terms of the images and kernels of the differential operators curl, div and grad, forms an exact sequence. This simplifies the later definition of appropriate potentials, that is, auxiliary fields used to rewrite Maxwell's equations (2.2) into different formulations that simplify their solving.

\subsection{Material Relations}

The fields in Maxwell's equations (2.2) are related to each other through the material relations [60], [61]

$$
\vec{D}=\varepsilon \vec{E}, \quad \overrightarrow{J_{\mathrm{c}}}=\boldsymbol{\sigma} \vec{E}, \quad \vec{H}=\boldsymbol{\nu} \vec{B} .
$$

Here, the permittivity $\varepsilon=\varepsilon\left(\|\vec{E}\|_{2}, \vec{r}\right)$, the conductivity $\sigma=\sigma\left(\|\vec{E}\|_{2}, \vec{r}\right)$ and the reluctivity $\boldsymbol{\nu}=\boldsymbol{\nu}\left(\|\vec{B}\|_{2}, \vec{r}\right)$ are rank-2 tensor fields $\mathbb{R} \times \Omega \rightarrow \mathbb{R}^{3 \times 3}$, with $\|\cdot\|_{2}$ being the Euclidean norm in space, and $\vec{J}_{\mathrm{c}}: \mathcal{I} \times \Omega \rightarrow \mathbb{R}^{3}$ the conduction current density. The inverse of those relations is described with the permeability $\boldsymbol{\mu}=\boldsymbol{\mu}\left(\|\vec{H}\|_{2}, \vec{r}\right)$ and the resistivity $\boldsymbol{\rho}=\boldsymbol{\rho}\left(\left\|\overrightarrow{J_{\mathrm{C}}}\right\|_{2}, \vec{r}\right)$, such that

$$
\vec{E}=\boldsymbol{\rho} \vec{J}_{\mathrm{c}}, \quad \vec{B}=\boldsymbol{\mu} \vec{H} .
$$

Remark 1. The field dependencies in (2.3) and (2.4) may be extended in case of having special material settings such as for example for the modelling of superconductivity [56], [65].

For simplicity of notation, the field and space dependencies of the materials are suppressed through the rest of the work. It is always stated explicitly if linear, i.e., field-independent, material laws are assumed. Otherwise, general non-linear material laws are considered.

The current density $\vec{J}$ in $(2.2 \mathrm{~b})$, can now be split into

$$
\vec{J}=\vec{J}_{\mathrm{c}}+\vec{J}_{\mathrm{s}}
$$

with $\vec{J}_{\mathrm{s}}: \mathcal{I} \times \Omega_{\mathrm{s}} \rightarrow \mathbb{R}^{3}$ being an external source current density, independent of the rest of the fields. It can for example be impressed by a stranded conductor [66] whose current arises from an exterior coupled circuit. 


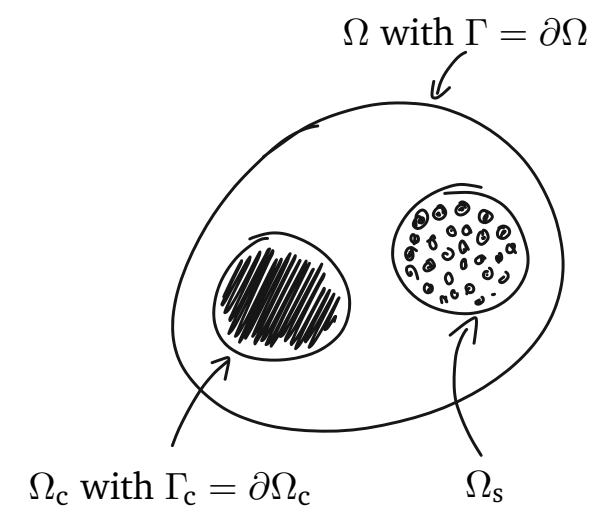

Figure 2.1: Sketch of the domain $\Omega$.

Note that $\partial / \partial t \vec{D}$ is the convection current density $\vec{J}_{\text {conv }}$ that is added to $\vec{J}$ to obtain the total current density

$$
\vec{J}_{\text {tot }}=\vec{J}_{\mathrm{c}}+\vec{J}_{\mathrm{s}}+\vec{J}_{\text {conv }}
$$

Assumption 2 (Subdomains). The domain $\Omega \subset \mathbb{R}^{3}$ may contain three types of subdomains, the conducting domain $\Omega_{\mathrm{c}}$, the (stranded) source domains $\Omega_{\mathrm{s}}^{(k)}, k=1, \ldots, n_{\mathrm{s}}$ and the remainder (excitation free) $\Omega_{0}$.

For a sketch of the domain and its possible subdomains see Figure 2.1.

Assumption 3 (Materials). The material tensors from relations (2.3) and (2.4) fulfil the following properties.

(a) The conductivity $\sigma$ and resistivity $\rho$ are symmetric positive definite in $\Omega_{\mathrm{c}}$ and zero elsewhere, that is,

$$
\operatorname{supp}(\boldsymbol{\sigma})=\Omega_{\mathrm{c}} \quad \operatorname{supp}(\boldsymbol{\rho})=\Omega_{\mathrm{c}} .
$$

(b) The reluctivity $\nu$, permeability $\boldsymbol{\mu}$ and permittivity $\varepsilon$ are symmetric positive definite in the entire domain $\Omega$.

\subsubsection{Nonlinear materials}

For non-linear materials, in addition to the chord reluctivity $\boldsymbol{\nu}$ and permeability $\boldsymbol{\mu}$ [67], also the differential reluctivity $\boldsymbol{\nu}_{\mathrm{d}}$ and permeability $\boldsymbol{\mu}_{\mathrm{d}}$,

$$
\begin{aligned}
& \boldsymbol{\nu}_{\mathrm{d}}(\mathbf{s})=\boldsymbol{\nu}(s) \mathbf{I}+\frac{1}{s} \frac{\partial \boldsymbol{\nu}(s)}{\partial s} \mathbf{s s}^{\top} \text { and } \\
& \boldsymbol{\mu}_{\mathrm{d}}(\mathbf{s})=\boldsymbol{\mu}(s) \mathbf{I}+\frac{1}{s} \frac{\partial \boldsymbol{\mu}(s)}{\partial s} \mathbf{s s}^{\top},
\end{aligned}
$$

can be defined, where $\mathbf{s} \in \mathbb{R}^{3}, s=\|\mathbf{s}\|_{2}$ and $\mathbf{I} \in \mathbb{R}_{3 \times 3}$ is the identity tensor. In order to state some important properties fulfilled by those differential materials, first some natural physical assumptions for the $\mathrm{B}-\mathrm{H}$ curve $B=f_{\mathrm{BH}}(H)$, where $H=\|\vec{H}\|_{2}$ and $B=\|\vec{B}\|_{2}$ are made.

Assumption 4 (BH-curve [68]). The BH-curve $f_{B H}(H)=\|\boldsymbol{\mu}(H) \vec{H}\|_{2}: \mathbb{R}_{0}^{+} \rightarrow \mathbb{R}_{0}^{+}$has the properties 
(a) $f_{\mathrm{BH}}(s)$ is continuously differentiable.

(b) $f_{\mathrm{BH}}(0)=0$.

(c) $f_{\mathrm{BH}}^{\prime}(s) \geq \mu_{0}, \forall s>0$.

(d) $\lim _{s \rightarrow \infty} f_{\mathrm{BH}}^{\prime}(s)=\mu_{0}$, with $\mu_{0}>0$ being the vacuum permeability.

Proposition 1 (Differential reluctivity (permeability)). Under Assumption 4 on the BH-curve and for nonlinearities only in the isotropic components of the reluctivity and permeability, the differential reluctivity and permeability are symmetric positive definite.

Proof. For a proof of this proposition see [69, Chapter 2] and [70, Chapter 3].

Like in the case of the BH-curve, also nonlinearities can arise on the EJ-curve and the ED-curve, which lead to the differential conductivity $\sigma_{\mathrm{d}}$, resistivity $\rho_{\mathrm{d}}$ and permittivity $\varepsilon_{\mathrm{d}}$

$$
\begin{aligned}
& \boldsymbol{\sigma}_{\mathrm{d}}(\mathbf{s})=\boldsymbol{\sigma}(s) \mathbf{I}+\frac{1}{s} \frac{\partial \boldsymbol{\sigma}(s)}{\partial s} \mathbf{s s}^{\top}, \\
& \boldsymbol{\rho}_{\mathrm{d}}(\mathbf{s})=\boldsymbol{\rho}(s) \mathbf{I}+\frac{1}{s} \frac{\partial \boldsymbol{\rho}(s)}{\partial s} \mathbf{s}^{\top} \text { and } \\
& \boldsymbol{\varepsilon}_{\mathrm{d}}(\mathbf{s})=\boldsymbol{\varepsilon}(s) \mathbf{I}+\frac{1}{s} \frac{\partial \boldsymbol{\varepsilon}(s)}{\partial s} \mathbf{s s}^{\top} .
\end{aligned}
$$

Assumption 5 (Differential conductivity (resistivity) and permittivity). We assume non-linearities only in the isotropic components of the material relation and the differential conductivity and resistivity to be symmetric positive definite on $\Omega_{\mathrm{c}}$. The differential permittivity is assumed to be symmetric positive definite on the entire domain $\Omega$.

Despite the lack of formalised theory regarding the EJ-curve and ED-curve, we take analogous assumptions to those of the BH-curve (Assumption 4), which are both mathematically as well as physically plausible and lead to Assumption 5. For an example of possible EJ-curves and ED-curves, see [71].

\subsection{Boundary and Initial Conditions}

The electromagnetic fields described by Maxwell's equations (2.2), exist in infinite domains and for all time $t \in \mathbb{R}$. However, in most simulations, they are solved in the truncated domain $\Omega$ described in Assumption 1 and on a time interval $\mathcal{I}=\left[t_{0}, t_{\text {end }}\right]$. For example, to imitate the behaviour of the fields on the infinite domain or to exploit symmetries on the considered geometry, appropriate BCs on $\Gamma=\partial \Omega$ are imposed.

Even though more elaborated BCs can be set, such as with shell transformations to mimic the infinite domain [72], we only consider homogeneous electric boundary conditions ('ebc'), which assumes an infinite conductivity at the boundary $(\sigma \rightarrow \infty)$ and homogeneous magnetic boundary conditions ('mbc'), which correspond to an infinite permeability $(\mu \rightarrow \infty)$ at the boundary [58], [60]. 


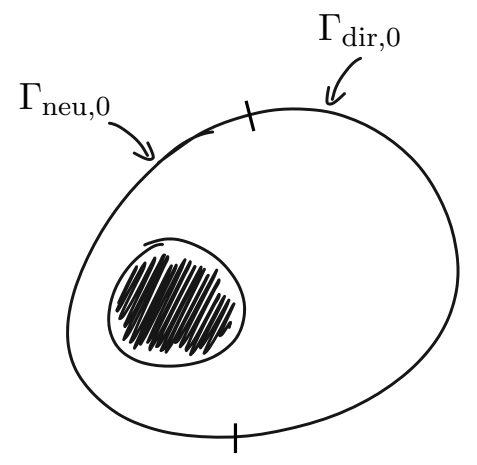

Figure 2.2: Sketch of the partition of the boundary $\Gamma=\Gamma_{\text {dir, } 0} \cup \Gamma_{\text {neu, } 0}$.

For $\vec{n}$ being the outward normal vector to the boundary $\Gamma=\Gamma_{\mathrm{ebc}} \cup \Gamma_{\mathrm{mbc}}$ with $\Gamma_{\mathrm{ebc}} \cap \Gamma_{\mathrm{mbc}}=\emptyset$, the BCs for all $t \in \mathcal{I}$ are

$$
\begin{cases}\vec{n} \times \vec{E}=0 & \text { in } \Gamma_{\mathrm{ebc}}, \\ \vec{n} \times \vec{H}=0 & \text { in } \Gamma_{\mathrm{mbc}} .\end{cases}
$$

Alternatively, for a given field $\vec{\xi}: \mathcal{I} \times \Omega \rightarrow \mathbb{R}^{3}$, the mathematical terminology homogeneous Dirichlet ('dir,0') and Neumann ('neu,0') BCs can be defined. Here, $\Gamma=\Gamma_{\text {dir, } 0} \cup \Gamma_{\text {neu, } 0}$ (see Figure 2.2) with $\Gamma_{\text {dir, } 0} \cap \Gamma_{\text {neu, } 0}=\emptyset$ and then, for all $t \in \mathcal{I}$

$$
\begin{cases}\vec{n} \times \vec{\xi}=0 & \text { in } \Gamma_{\mathrm{dir}, 0}, \\ \vec{n} \times(\operatorname{curl} \vec{\xi})=0 & \text { in } \Gamma_{\text {neu }, 0} .\end{cases}
$$

Electrical engineers prefer the terminology electric or magnetic boundary conditions, which is related to the physical behaviour of the fields and independent of the chosen formulation, that is, the variables that describe the problem. The relation between the two terminologies for the different formulations is explained later if necessary.

In addition to the BCs in space, also ICs in time have to be set at the starting point $t_{0}$. That is, for the given field $\vec{\xi}(t, \vec{r})$, the condition

$$
\vec{\xi}\left(t_{0}, \vec{r}\right)=\vec{\xi}_{0}, \quad \text { for all } \vec{r} \in \Omega
$$

is enforced. For consistency, the initial conditions are chosen to be a possible solution of the partial differential equation.

\subsection{Static and Quasistatic Fields}

Maxwell's equations describe three different physical effects; the electric, magnetic and Ohmic losses. For some problem settings, part of these effects can be neglected with respect to the others. This leads to approximations to Maxwell's equations which can be classified [73] as follows.

Definition 1 (Approximations [58]). The different approximations of Maxwell's equations (2.2) are 
(a) static if the time derivative of the magnetic and electric flux densities are disregarded, i.e.

$$
\frac{\partial}{\partial t} \vec{B}=0 \quad \text { and } \quad \frac{\partial}{\partial t} \vec{D}=0
$$

Here, the conduction current density $\vec{J}_{\mathrm{c}}=0$ and thus the electric and magnetic fields can be entirely decoupled. There are two different settings that can be considered. In the electrostatic (ES) case only electric or capacitive effects are described and in the magnetostatic (MS) approximation only magnetic or inductive effects are studied.

(b) electroquasistatic (EQS), if only the time differentiation of the magnetic flux density is neglected

$$
\frac{\partial}{\partial t} \vec{B}=0
$$

Under this approximation, the inductive effects are disregarded.

(c) magnetoquasistatic (MQS) if only the time differentiation of the electric flux density is neglected

$$
\frac{\partial}{\partial t} \vec{D}=0
$$

In this case, the capacitive effects are not considered.

(d) full wave if all effects are considered and thus no approximations are made.

For some applications, the simplifications above describe the electromagnetic fields with enough accuracy and thus can be used instead of the full Maxwell's equations. This results into easier solvable PDEs, as second timederivatives are avoided and the computation of only one field, electric or magnetic, is needed. The most suitable approximation can be chosen depending on the given problem and might depend on properties such as the materials, the frequencies or the spatial dimensions of the geometry [74]-[76].

One last approximation, which is less common and can be though of as a combination of c) and b), is the Darwin model [77], [78]. Here, electric, magnetic and ohmic loss effects are described, but wave propagation is neglected.

\subsection{Formulations}

Once a suitable approximation (see Definition 1) for Maxwell's equations has been chosen, typically the remaining equations are combined into a formulation by defining potentials. Depending on the used approximation, different possibilities arise.

\subsubsection{Full Maxwell}

Even though, when considering Maxwell's equations without simplifications, that is, Definition 1.d), also potentials can be defined (see [20]), often no potentials are used and the so-called $\vec{E}-\vec{H}$ formulation is performed [79]. 
Assuming that the initial conditions fulfil the divergence relations of Maxwell's equations (2.2d) and (2.2c), only Faraday's (2.2a) and Ampère (2.2b) law need to be considered. After using the material relations (2.3)(2.5), the PDEs are rewritten as

$$
\frac{\partial}{\partial t} \boldsymbol{\mu} \vec{H}+\operatorname{curl} \vec{E}=0 \quad \text { and } \quad-\frac{\partial}{\partial t} \varepsilon \vec{E}-\operatorname{curl} \vec{H}-\boldsymbol{\sigma} \vec{E}=-\vec{J}_{\mathrm{s}},
$$

which yields the system of equations for the $\vec{E}-\vec{H}$ formulation. The BCs are set directly for $\vec{E}$ and $\vec{H}$ on $\Gamma_{\mathrm{ebc}}$ and $\Gamma_{\mathrm{mbc}}$ as described in (2.7).

\subsubsection{Electroquasistatics}

In an electroquasistatic setting, that is, Definition 1.b), the time derivative of the magnetic flux density in Faraday's law (2.2a) is neglected $(\partial \vec{B} / \partial t=0)$. This simplified equation, together with the divergence of Ampère's law (2.2b) yields the two governing PDEs for EQS

$$
\operatorname{curl} \vec{E}=0 \quad \operatorname{div} \frac{\partial}{\partial t} \vec{D}+\operatorname{div} \vec{J}=0 .
$$

Remark 2. We do not refer to equation (2.2c), as we generally consider problems, in which the charge distribution is unknown. Please note that this quantity is linked to our system by means of the continuity equation [58]

$$
\operatorname{div} \vec{J}+\frac{\partial}{\partial t} \rho=0
$$

Using the fact that the electric field is curl-free in (2.11) and for topologically trivial domains (see Assumption 1), $\vec{E}$ can be rewritten as the gradient of an electric scalar potential $\phi: \mathcal{I} \times \Omega \rightarrow \mathbb{R}$

$$
\vec{E}=-\operatorname{grad} \phi .
$$

The equation for electroquasistatics is obtained in terms of the scalar potential by exploiting the material relations (2.3) and reads

$$
\frac{\partial}{\partial t} \operatorname{div} \varepsilon \operatorname{grad} \phi+\operatorname{div} \sigma \operatorname{grad} \phi=0 .
$$

Typically, for EQS applications, homogeneous electric BCs are set, which, for the degrees of freedom $\phi$ yields

$$
\vec{n} \times \operatorname{grad} \phi=0, \text { on } \Gamma_{\mathrm{ebc}} .
$$

\subsubsection{Magnetoquasistatics}

For the magnetoquasistatic approximation, as stated in Definition 1.c), the displacement current is neglected in Ampère's law (2.2b) $(\partial \vec{D} / \partial t=0)$ which, in combination with (2.2a) and (2.2d) form the three PDEs for magnetoquasistatics

$$
\operatorname{curl} \vec{E}=-\frac{\partial \vec{B}}{\partial t} \quad \operatorname{curl} \vec{H}=\vec{J}_{\mathrm{c}}+\vec{J}_{\mathrm{s}} \quad \operatorname{div} \vec{B}=0 .
$$

In this case, different potentials can be defined which lead to distinct formulations. This can be exploited, as they might have different advantages depending on the setting. 


\subsection{4 $\vec{A}-\phi$ formulation}

The first approach which is considered is the $\vec{A}-\phi$ formulation [64], [80], [81]. In this case, a magnetic vector potential $\vec{A}: \mathcal{I} \times \Omega \rightarrow \mathbb{R}^{3}$ and an electric scalar potential $\phi: \mathcal{I} \times \Omega \rightarrow \mathbb{R}$ are defined by exploiting the divergence-freeness of the magnetic flux density $\vec{B}$ in (2.15) and integrating Faraday's law (2.2a) in space, such that

$$
\vec{B}=\operatorname{curl} \vec{A} \quad \text { and } \quad \vec{E}=-\frac{\partial \vec{A}}{\partial t}-\operatorname{grad} \phi .
$$

Here, the magnetic vector potential defines the magnetic flux density $\vec{B}$ only up to a gradient field. Therefore, in regions with zero conductivity $\left(\vec{J}_{\mathrm{c}}=0\right)$, a gauging condition is required so as to ensure uniqueness of solution [81]-[83].

Remark 3. Notice that, in order to define the potentials (2.16) the simplifications made for MQS (2.15) are not necessary. Therefore, this formulation can also be used to describe problems involving the full set of Maxwell's equations (2.2) [20]. However, this formulation, in contrast to the $\vec{E}-\vec{H}$ one, requires a gauging condition as well as an auxiliary variable to avoid second time derivatives of the magnetic vector potential. In practice it is mainly used in the context of semiconductors (cf. [84]).

The different boundary conditions introduced in Section 2.3 can now be translated into expressions involving only the potentials.

$$
\begin{cases}\vec{n} \times \vec{A}=0, \phi=0 & \text { on } \Gamma_{\mathrm{ebc}} \\ \vec{n} \times(\boldsymbol{\nu} \operatorname{curl} \vec{A})=0, & \text { on } \Gamma_{\mathrm{mbc}} .\end{cases}
$$

Therefore, in this formulation, electric boundary conditions correspond to Dirichlet boundary conditions for $\vec{A}$ and $\phi$ and magnetic boundary conditions result into Neumann boundary conditions for $\vec{A}$.

As we consider magnetic vector potential formulations only for magnetoquasistatic problems, one additional simplification can be made, which leads to the $\vec{A}^{*}$ formulation.

\subsubsection{1 $\vec{A}^{*}$ formulation}

To obtain the $\vec{A}^{*}$ formulation, the gauging freedom of the $\vec{A}-\phi$ formulation is exploited, which allows to choose a specific magnetic vector potential, thereby reducing the degrees of freedom [85]. This is achieved by defining an alternative vector potential $\vec{A}^{*}: \mathcal{I} \times \Omega \rightarrow \mathbb{R}^{3}$, such that

$$
\vec{A}^{*}(t, \vec{r})=\vec{A}(t, \vec{r})+\int_{s=t_{0}}^{t} \operatorname{grad} \phi(s, \vec{r}) \mathrm{d} s .
$$

If this is now inserted into (2.16), the scalar potential vanishes from both equalities, i.e.,

$$
\vec{B}=\operatorname{curl} \vec{A}^{*}=\operatorname{curl} \vec{A} \quad \text { and } \quad \vec{E}=-\frac{\partial}{\partial t} \vec{A}-\operatorname{grad} \phi=-\frac{\partial}{\partial t} \vec{A}^{*} .
$$

Again, to ensure a unique solution in non-conducting regions, another gauging condition is still required. For simplicity of notation we will from now own overload the symbol $\vec{A}$ and use it also for the magnetic vector potential in the $\vec{A}^{*}$ formulation. 
Maxwell's equations for MQS (2.15) and the material relations (2.3) leads to the following curl-curl equation for magnetoquasistatics, also called eddy current equation, in terms of the defined potentials

$$
\boldsymbol{\sigma} \frac{\partial}{\partial t} \vec{A}+\operatorname{curl} \boldsymbol{\nu} \operatorname{curl} \vec{A}=\overrightarrow{J_{\mathrm{s}}} .
$$

Analogous to the $\vec{A}-\phi$ formulation, the boundary conditions for the magnetic vector potential read

$$
\begin{cases}\vec{n} \times \vec{A}=0, & \text { on } \Gamma_{\mathrm{ebc}}, \\ \vec{n} \times(\boldsymbol{\nu} \operatorname{curl} \vec{A})=0, & \text { on } \Gamma_{\mathrm{mbc}} .\end{cases}
$$

\subsection{5 $\vec{T}-\Omega$ formulation}

The second approach is the $\vec{T}-\Omega$ formulation [86]-[88]. In this case, an electric vector potential $\vec{T}$ : $\mathcal{I} \times \Omega_{\mathrm{c}} \rightarrow \mathbb{R}^{3}$ only in the conducting domain and a global magnetic scalar potential $\psi: \mathcal{I} \times \Omega \rightarrow \mathbb{R}$ are defined such that

$$
\vec{J}_{\mathrm{c}}=\operatorname{curl} \vec{T} \quad \text { and } \quad \vec{H}=\vec{H}_{\mathrm{s}}+\vec{T}-\operatorname{grad} \psi .
$$

Here, $\vec{H}_{\mathrm{s}}: \mathcal{I} \times \Omega \rightarrow \mathbb{R}^{3}$ is an auxiliary source magnetic field with

$$
\operatorname{curl} \vec{H}_{\mathrm{s}}=\vec{J}_{\mathrm{s}} \text {. }
$$

In this case, an additional gauging condition is required for $\vec{T}$ to ensure uniqueness of solution.

The combination of Maxwell's equations for MQS (2.15) with the materials laws (2.4) in terms of the potentials (2.22) yield the system of PDEs for the $\vec{T}-\Omega$ formulation

$$
\begin{aligned}
\operatorname{curl} \boldsymbol{\rho} \operatorname{curl} \vec{T}+\frac{\partial}{\partial t} \boldsymbol{\mu} \vec{T}-\frac{\partial}{\partial t} \boldsymbol{\mu g r a d} \psi & =-\frac{\partial}{\partial t} \boldsymbol{\mu} \vec{H}_{\mathrm{s}} \\
\operatorname{div} \boldsymbol{\mu} \vec{T}-\operatorname{div} \boldsymbol{\mu} \operatorname{grad} \psi & =-\operatorname{div} \boldsymbol{\mu} \vec{H}_{\mathrm{s}} .
\end{aligned}
$$

Remark 4. In the $\vec{A}^{*}$ formulation, the equation for no magnetic monopoles (2.2d) is immediately fulfilled by the choice of the magnetic vector potential and Faraday's law (2.2a) is used to describe $\vec{E}$ in terms of the potential. Ampère's law (2.2b), however, has to be solved explicitly by the the eddy current equation (2.20). On the contrary, in the $\vec{T}-\Omega$ formulation, the definition of the potentials immediately fulfils Ampère's law (2.2b) and both the equation for no magnetic monopoles (2.2d) as well as Faraday's law (2.2a) have to be described explicitly by the PDE in (2.24).

Again the boundary conditions from Section 2.3 can be expressed in terms of the defined potentials as

$$
\begin{cases}\mu \frac{\partial \psi}{\partial \vec{n}}=0, & \text { on } \Gamma_{\mathrm{ebc}}, \\ \vec{n} \times \operatorname{grad} \psi=0, & \text { on } \Gamma_{\mathrm{mbc}} .\end{cases}
$$

Neumann boundary conditions for $\psi$ are obtained for electric boundary conditions and magnetic boundary conditions yield Dirichlet boundary conditions for $\operatorname{grad} \psi$. 


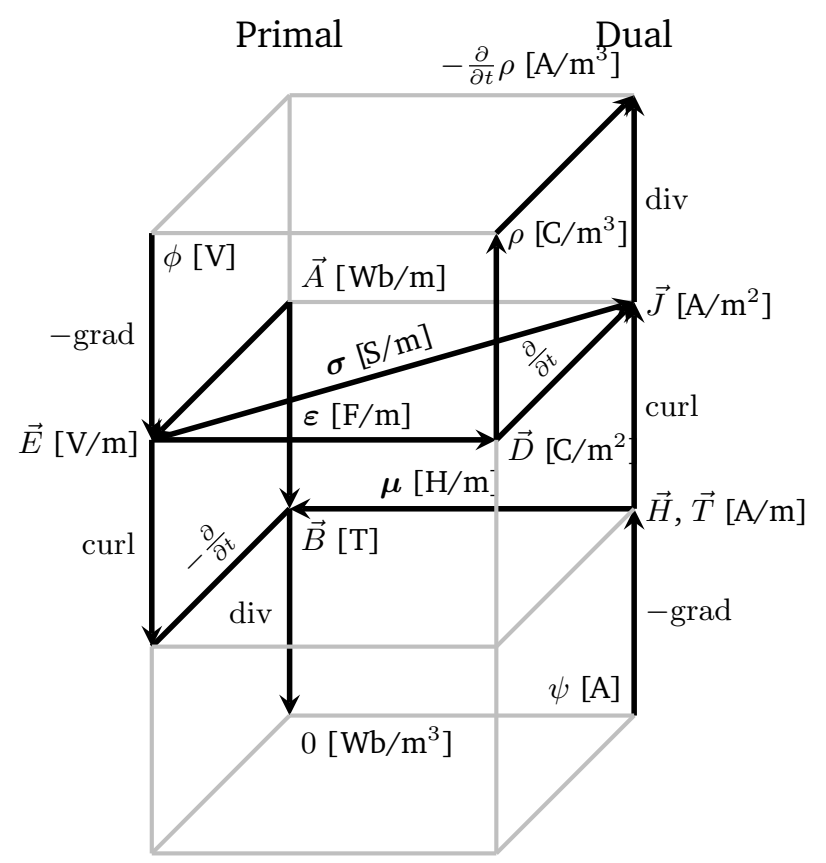

Figure 2.3: Maxwell house based on [64].

Since the electric vector potential $\vec{T}$ is only defined on $\Omega_{\mathrm{c}}$, additional boundary conditions for $\vec{T}$ have to be set in $\Gamma_{\mathrm{c}}=\partial \Omega_{\mathrm{c}}$. To ensure tangential continuity of the electric field, electric boundary conditions are required. This translates into the following Dirichlet boundary condition

$$
\vec{n}_{\mathrm{c}} \times \rho \vec{T}=0 \quad \text { on } \Gamma_{\mathrm{c}},
$$

where $\vec{n}_{\mathrm{c}}$ is the outward normal unit vector of $\Gamma_{\mathrm{c}}$.

\subsubsection{Duality of the formulations}

A diagram to visualise the relation between the different field quantities and potentials defined for Maxwell's equations and its approximations, as well as the material relations, can be found in Figure 2.3. This is called Maxwell's House [89], [90] or Tonti diagram [91]. Here, the potentials for the $\vec{A}-\phi$ (or $\vec{A}^{*}$ ) and the ones for the $\vec{T}-\Omega$ formulation are seen to be located on dual (or opposite) sides of the diagram. However, they can be used to physically describe the same setting. These type of formulations are called dual or complementary and can for example be exploited for an error approximation of the space discretisation method [92].

\subsection{Modelling of Excitations}

The excitation of the formulations in Section 2.5 is typically given by either voltage drops $v_{k}: \mathcal{I} \rightarrow \mathbb{R}$ or lumped currents $i_{k}: \mathcal{I} \rightarrow \mathbb{R}$. These are zero dimensional functions, that have to be coupled to the three dimensional PDEs obtained with the different formulations (see Section 2.5). We consider two different coupling approaches, the excitation with characteristic functions (winding density functions [66]) or with non-homogeneous boundary conditions [20]. 


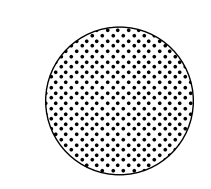

Figure 2.4: Sketch of stranded conductor cable's cross-section.

\subsubsection{Excitation with winding density functions}

One possible coupling is through the known source current density $\vec{J}_{\mathrm{s}}$ in $(2.5)$, which can be linked to $v_{k}$ or $i_{k}$ by means of so-called conductor models. Different models, such as the solid and stranded conductors [31], or foil-conductor models [93] are proposed. In [66] winding density functions are defined, which set up a framework to describe stranded, solid and foil conductor models. The functions are denoted by

$$
\vec{\chi}_{k}: \Omega \rightarrow \mathbb{R}^{3}
$$

Remark 5. We will only focus on the most common stranded conductor model, as it has been shown that in-space discretised solid and stranded conductor models are structurally equivalent [94] in the case of the $\vec{A}^{*}$-formulation.

Definition 2 (Stranded conductor model). For stranded conductors, the winding density functions $\vec{\chi}_{k}$ are defined such that

$$
\vec{J}_{\mathrm{s}}^{(k)}=\vec{\chi}_{k} i_{k}, \quad \forall k=1, \ldots, n_{\mathrm{s}}
$$

where $\vec{J}_{\mathrm{s}}^{(k)}$ is the source current density function in the $k$-th conductor.

Assumption 6 (Disjoint subdomains). Given $n_{\mathrm{s}}$ stranded conductor excitations in different source domains $\Omega_{\mathrm{s}}^{(k)}$, $k=1 \ldots n_{\mathrm{s}}$, with $\Omega_{\mathrm{s}}=\bigcup_{k=1}^{n_{\mathrm{s}}} \Omega_{\mathrm{s}}^{(k)}$ and $\operatorname{supp} \vec{\chi}_{k}=\Omega_{\mathrm{s}}^{(k)}$, then the subdomains of Assumption 2 are disjoint, i.e.

$$
\Omega_{\mathrm{c}} \cap \Omega_{\mathrm{s}}^{(k)}=\emptyset, \forall k \quad \text { and } \quad \Omega_{\mathrm{s}}^{(k)} \cap \Omega_{\mathrm{s}}^{(p)}, \text { for } k \neq p
$$

This assumption arises from the physical situation where many individual strands with a small diameter form the conductor (see the sketch in Figure 2.4). Here, a homogeneous current distribution is assumed [31], [66], justified by the fact that the diameter of each strand is smaller than the skin depth and thus the eddy currents can be neglected. Therefore, the stranded conductor domain $\Omega_{\mathrm{s}}$ is not considered as part of the conducting domain $\Omega_{\mathrm{c}}$, where the eddy currents are present and, mathematically, this can be translated into $\Omega_{\mathrm{c}}$ and $\Omega_{\mathrm{s}}$ being disjoint. The advantage of this homogenisation model is that it avoids having to spatially resolve the individual strands, which would lead to high computational effort.

Furthermore, we consider conductor models that do not intersect [22], which yields the second part of the assumption. With these properties, we have

$$
\operatorname{supp}\left(\vec{\chi}_{k}\right)=\Omega_{\mathrm{s}}^{(k)} \quad \text { and } \quad \vec{\chi}_{i} \cdot \vec{\chi}_{j}=0 \quad \text { for } i \neq j .
$$


In [66], an important partition of unity property of density winding functions is presented. Therefore, if $A_{k}$ is a cross-sectional plane of the $k$-th stranded conductor, then

$$
\int_{A_{k}} \vec{\chi}_{k} \cdot \mathrm{d} \vec{S}=N_{k}, \quad \forall A_{k}
$$

where $N_{k}$ is the number of turns of the winding.

In addition, winding density functions also allow to establish a relation between voltage drops $v_{k}$ and the field quantities. For the stranded conductor case we have [66]

$$
v_{k}=-\int_{\Omega} \vec{\chi}_{k} \cdot \vec{E} \mathrm{~d} V
$$

This last property can be exploited to both obtain the voltage drop as a post-processing step as well as excite the field problem with given voltages. Furthermore, it generates an implicit relation between currents and voltages that can be used to couple the three (or two) dimensional field problems to lumped circuit equations [95].

Remark 6. In the literature, different proposals can be found on how to construct the stranded conductor winding density functions. A common approach is to solve a Laplace-type problem on the subdomain $\Omega_{\mathrm{s}}^{(k)}$ (see [66], [96], [97]).

Definition 3 (Source current density coupling). The source current density in (2.5) is

$$
\overrightarrow{J_{\mathrm{s}}}=\sum_{k=1}^{n_{\mathrm{s}}} \vec{\chi}_{k} i_{k}=\chi_{\mathrm{s}} \mathbf{i}
$$

where $\chi_{\mathrm{s}}: \Omega_{\mathrm{s}} \rightarrow \mathbb{R}^{3 \times n_{\mathrm{s}}}$ is a rank-2 tensor field that collects all winding functions and $\mathbf{i}: \mathcal{I} \rightarrow \mathbb{R}^{n_{\mathrm{s}}}$ is a vector-valued functions containing the currents across all the conductors.

Due to Ampère's law (2.2b), the total current density

$$
\vec{J}_{\mathrm{tot}}=\vec{J}_{\mathrm{c}}+\frac{\partial \vec{D}}{\partial t}+\vec{J}_{\mathrm{s}}
$$

has to be divergence-free, that is, $\operatorname{div} \vec{J}_{\text {tot }}=0$. This does not imply that the source current density $\vec{J}_{\mathrm{S}}$ is solenoidal too. However, in most models this is the case. For example, in the non-conducting region of a magnetoquasistatic setting, $\vec{J}_{\mathrm{s}}$ has to be divergence free, so as to be a valid right-hand-side for Ampère's law in magnetoquasistatics (2.15). Under those circumstances, $\vec{J}_{\mathrm{s}}$ can be written in terms of a source magnetic field strength

$$
\overrightarrow{J_{\mathrm{s}}}=\operatorname{curl} \vec{H}_{\mathrm{s}},
$$

which constitutes the source field for the $\vec{T}-\Omega$ formulation (2.24).

Definition 4 (Source magnetic field coupling). The source magnetic field strength in (2.22) is

$$
\vec{H}_{\mathrm{s}}=\zeta_{\mathrm{s}} \mathbf{i}
$$

where $\zeta_{\mathrm{s}}: \Omega \rightarrow \mathbb{R}^{3 \times n_{\mathrm{s}}}$ is a rank-2 tensor field. 


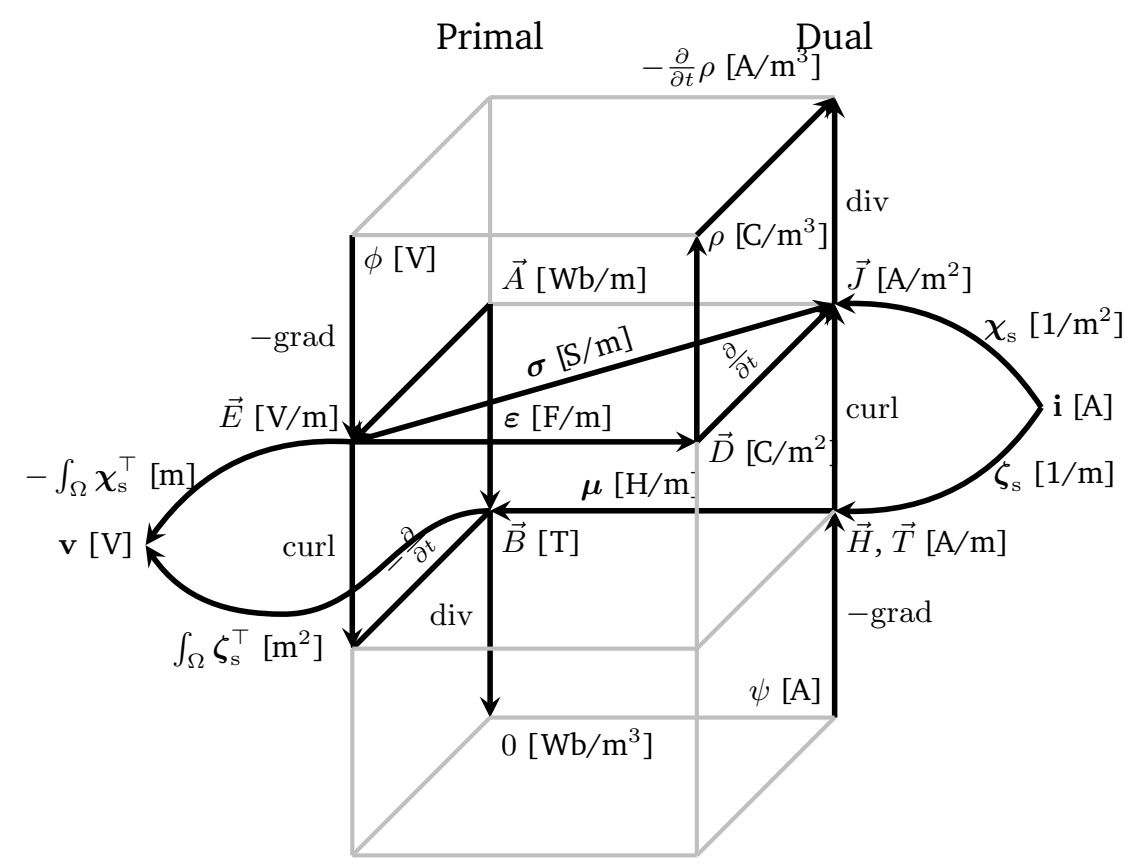

Figure 2.5: Maxwell house's extension with winding density functions [55].

Due to the relation between the source current density and the source magnetic field strength (2.23), we have for the winding density functions that

$$
\vec{\chi}_{\mathrm{s}}^{(k)}=\operatorname{curl} \vec{\zeta}_{\mathrm{s}}^{(k)}
$$

with $\vec{\zeta}_{\mathrm{s}}^{(k)}$ being the $k$-th column of $\zeta_{\mathrm{s}}$.

The voltage drop along the $k$-th conductor $v_{k}$ for a setting with electric BC (2.7) is described by (see [55], [98])

$$
v_{k}=\frac{\mathrm{d}}{\mathrm{d} t} \int_{\Omega} \vec{\zeta}_{\mathrm{s}}^{(k)} \cdot \vec{B} \mathrm{~d} \Omega .
$$

This follows immediately from applying Gauss's theorem and Faraday's law (2.2a) as well as the relation between the two winding density functions (2.32) to the definition of voltage drop across a stranded conductor (2.29).

In Figure 2.5, an extension of Maxwell's house is made, that considers the winding density functions and excitations (see [55]).

\subsubsection{Excitation with boundary conditions}

Another common approach of exciting the problem is by setting inhomogeneous Dirichlet BC that impose specific voltage $v_{k}$ or current $i_{k}$ values on parts of the boundary.

Assumption 7 (Disjoint boundary decomposition). The boundary of the domain $\Omega$ fulfilling Assumption 1 can be decomposed into three disjoint subsets, such that, for $\Gamma=\partial \Omega$,

$$
\Gamma=\Gamma_{\mathrm{s}} \cup \Gamma_{\text {dir }, 0} \cup \Gamma_{\text {neu }, 0},
$$




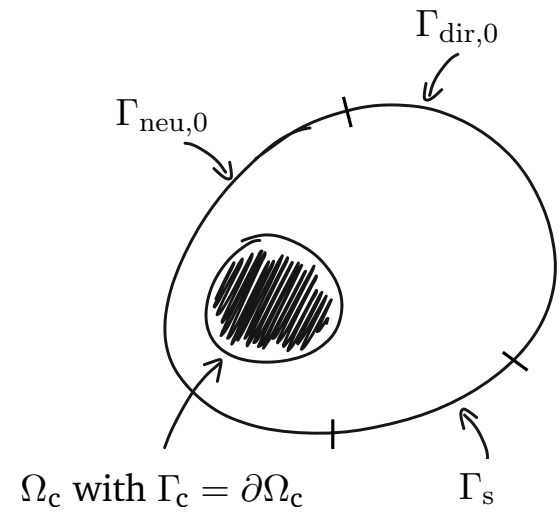

Figure 2.6: Sketch of the partition of the boundary $\Gamma$.

where $\Gamma_{\mathrm{s}}$ is the source boundary.

A sketch of the domain considered in such settings can be found in Figure 2.6. The only examples excited with boundary conditions that appear during this work are in an electroquasistatic setting. Therefore, we will consider the electroquasistatic PDE (2.13) to illustrate how such boundary condition excitations can be formally described in a mathematical setting.

We consider the electroquasistatic PDE (2.13) with homogeneous Dirichlet BC on $\Gamma_{\mathrm{dir}, 0}$, homogeneous Neumann BC on $\Gamma_{\text {neu, }, 0}$ and voltage excitation $v_{k}$ on $n_{\mathrm{s}}+1$ ports located at the boundary $\Gamma_{\mathrm{s}}$. For each port $k$, we define potentials $e_{k}: \mathcal{I} \rightarrow \mathbb{R}$, such that $v_{k}=e_{k}-e_{0}$. Here, $e_{0}$ is the reference potential and the particular choice $e_{0}=0$ is taken. The source boundary $\Gamma_{\mathrm{s}}$ is divided into disjoint subsets, such that $\Gamma_{\mathrm{s}}=\Gamma_{\mathrm{s}}^{(0)} \cup \ldots \cup \Gamma_{\mathrm{s}}^{\left(n_{\mathrm{s}}\right)}$, with $\Gamma_{\mathrm{s}}^{(i)} \cap \Gamma_{\mathrm{s}}^{(j)}=\emptyset$, for $i \neq j$. Now we can define the boundary value problem

$$
\begin{aligned}
\frac{\partial}{\partial t} \operatorname{div} \varepsilon \operatorname{grad} \phi+\operatorname{div} \sigma \operatorname{grad} \phi & =0 & & \text { in } \Omega, \\
\phi & =0 & & \text { on } \Gamma_{\mathrm{dir}, 0}, \\
\phi & =e_{k} & & \text { on } \Gamma_{\mathrm{s}}, \\
\frac{\partial \phi}{\partial \vec{n}} & =0 & & \text { on } \Gamma_{\text {neu }, 0},
\end{aligned}
$$

where $\vec{n}$ is the vector normal to $\Gamma_{\text {neu }, 0}$.

This setting can be-rewritten in terms of a Dirichlet lift Ansatz, which allows to obtain a structure similar to the one of the winding density functions explained in Section 2.6.1.

Definition 5 (Lift function). For each port $k=1, \ldots, n_{\mathrm{s}}+1$ we choose the lift function $\Upsilon_{k}: \Omega \rightarrow \mathbb{R}$ with

$$
\Upsilon_{k_{\mid \Gamma}}= \begin{cases}1, & \text { for } \vec{r} \in \Gamma_{\mathrm{s}}^{(k)} \\ 0, & \text { otherwise }\end{cases}
$$

and being the solution of e.g. div $\operatorname{grad} \Upsilon_{k}=0$. 


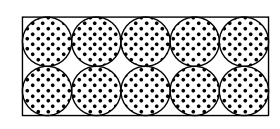

Figure 2.7: Sketch of Rutherford cable's cross-section.

Definition 6 (Source electric scalar potential). We define the source electric scalar potential $\phi_{\mathrm{s}}: \mathcal{I} \times \Omega \rightarrow \mathbb{R}$ as

$$
\phi_{\mathrm{s}}=\sum_{k=1}^{n_{\mathrm{s}}} \Upsilon_{k} e_{k}=\Upsilon \mathbf{e}
$$

with $\mathbf{e}: \mathcal{I} \rightarrow \mathbb{R}^{n_{\mathrm{s}}}$ the vector of potentials and $\Upsilon: \Omega \rightarrow \mathbb{R}^{3 \times n_{\mathrm{s}}}$ a rank-2 tensor field containing the lift functions as columns.

Now we can re-write the electroquasistatic boundary value problem (2.34) with inhomogeneous BCs as a homogeneous boundary value problem

$$
\begin{aligned}
\frac{\partial}{\partial t} \operatorname{div} \varepsilon \operatorname{grad} \phi+\operatorname{div} \sigma \operatorname{grad} \phi & =-\frac{\partial}{\partial t} \operatorname{div} \varepsilon \operatorname{grad} \phi_{\mathrm{s}}-\operatorname{div} \sigma \operatorname{grad} \phi_{\mathrm{s}} & & \text { in } \Omega \\
\phi & =0 & & \text { in } \Gamma_{\text {dir }} \\
\frac{\partial \phi}{\partial \vec{n}} & =0 & & \text { in } \Gamma_{\text {neu }, 0},
\end{aligned}
$$

with $\Gamma_{\text {dir }}=\Gamma_{\text {dir }, 0} \cup \Gamma_{\mathrm{s}}$. Analogously to the winding density functions, the lift functions allow us to establish a relation between the currents $i_{k}$ through the ports and the field quantities as

$$
i_{k}=\int_{\Omega} \operatorname{grad} \Upsilon_{k} \cdot \vec{J}_{\text {tot }} \mathrm{d} \Omega
$$

Remark 7. Expression (2.37) follows from the definition of current through the $k$-th port as

$$
i_{k}=\int_{\Gamma_{k}} \vec{J}_{\mathrm{tot}} \cdot \mathrm{d} \vec{A}
$$

Due to the definition of lift functions (Definition 5), this can be re-written as $i_{k}=\int_{\partial \Omega} \Upsilon_{k} \vec{J}_{\mathrm{tot}} \cdot \mathrm{d} \vec{A}$ and by applying Gauss's theorem, expression (2.37) is obtained.

\subsection{Modelling of Superconducting Magnets}

The Rutherford cables used for the coil of the superconducting magnets have a multiscale, heterogeneous and rectangular cross-section (see Figure 2.7) consisting of several strands surrounded by a filling material with an insulation layer. Each one of these strands embeds superconducting filaments into a wire of normal conducting material (copper) [99].

Computing the cable eddy currents with classical Maxwell's equations would require the micrometer [99] scale of the superconducting filaments to be resolved, which leads to unacceptably high computational cost. Therefore, a homogenisation model is used. For all the simulations of superconducting magnets in this work, we only consider simulations of the two dimensional magnet's cross-section as depicted in Figure 2.7 . 


\subsubsection{D homogenisation model}

The homogenisation model [8], [100] treats the eddy currents in a cable magnetisation term $\vec{M}_{\mathrm{s}}: \mathcal{I} \times \Omega \rightarrow \mathbb{R}^{3}$, which appears in the the curl-curl equation for magnetoquasistatics (2.20) as

$$
\sigma \frac{\partial}{\partial t} \vec{A}+\operatorname{curl} \nu \operatorname{curl} \vec{A}=\vec{J}_{\mathrm{s}}+\operatorname{curl} \vec{M}_{\mathrm{s}}
$$

We model inter-filament coupling currents, that is, the eddy currents that arise due to the normal conducting matrix in which the superconducting filaments are placed [65]. In this case, the magnetisation is computed by means of [100]

$$
\vec{M}_{\mathrm{s}}=-\nu \tau_{\mathrm{eq}} \frac{\partial}{\partial t} \vec{B}
$$

where $\tau_{\text {eq }}\left(\|\vec{B}\|_{2}, \vec{r}\right)$ is an equivalent cable time constant, which depends on parameters of the cable [99] and the magnitude of the magnetic field density $\vec{B}$. Also, other types of eddy currents can arise such as the interstrand coupling currents [65], however, those will not be considered in this model.

Assumption 8 (Equivalent cable time constant). The equivalent cable time constant $\tau_{\mathrm{eq}}$ is positive definite in the source domains $\Omega_{\mathrm{s}}$ and zero everywhere else, that is

$$
\operatorname{supp}\left(\tau_{\text {eq }}\right)=\Omega_{\mathrm{s}} .
$$

After inserting the homogenisation term, the eddy current (or curl-curl) equation for magnetoquasistatics in superconducting cables is

$$
\operatorname{curl} \nu \tau_{\text {eq }} \operatorname{curl} \frac{\partial}{\partial t} \vec{A}+\sigma \frac{\partial}{\partial t} \vec{A}+\operatorname{curl} \nu \operatorname{curl} \vec{A}=\vec{J}_{\mathrm{s}} .
$$

Note that, the classical eddy current term $\sigma \frac{\partial}{\partial t} \vec{A}$ may be zero, if no normal conducting eddy currents are modelled. This, however, still yields a dynamic PDE with time-derivatives involved due to the equivalent magnetisation term.

\subsubsection{Heat Equation}

When simulating quench propagation in superconducting accelerator magnets, being able to model the heat dynamics is of high importance, as this is one of the key quantities that characterise the creation and evolution of the quench inside the magnet and thus the effectiveness of the protection system. The temperature dynamics on the superconducting coils' domain $\Omega_{\mathrm{s}}$ is described by the heat balance equation [56], [65]

$$
\rho_{\mathrm{T}} C_{\mathrm{p}} \frac{\partial}{\partial t} T-\operatorname{div} k \operatorname{grad} T=P_{\mathrm{s}}+P_{\text {Joule }} .
$$

Here $\rho_{\mathrm{T}}$, the mass density, $C_{\mathrm{p}}$, the heat capacity and $k$ the thermal conductivity are scalar values that depend on the materials and $T: \mathcal{I} \times \Omega_{\mathrm{s}} \rightarrow \mathbb{R}$ is the temperature. The right hand side contains the Joule losses [56], [65]

$$
P_{\text {Joule }}=q_{\text {flag }} \sigma^{-1}\left\|\vec{J}_{\mathrm{s}}\right\|_{2}^{2},
$$

where $\sigma\left(\|\vec{E}\|_{2}\right)$ is a homogenised nonlinear conductivity and $q_{\mathrm{flag}}\left(\vec{B}, \overrightarrow{J_{\mathrm{s}}}, t\right)$ is the quench flag, a sigmoid-type function which is non-zero only in case of a quench. The power density

$$
P_{\mathrm{s}}=-\vec{M}_{\mathrm{s}} \cdot \frac{\partial}{\partial t} \vec{B}
$$


couples the heat equation with the electromagnetic field solution. Furthermore, on the quenched state, an Ohmic resistance on the superconducting coils $\mathbf{v}=\mathbf{R}_{\mathrm{s}} \mathbf{i}$ can be extracted [56], such that

$$
\mathbf{R}_{\mathrm{s}}=q_{\text {flag }} \int_{\Omega_{\mathrm{s}}} \chi_{\mathrm{s}}^{\top} \sigma^{-1} \chi_{\mathrm{s}} \mathrm{d} \Omega
$$

On the boundary of the domain where the heat equation is solved $\Gamma_{\mathrm{s}}=\partial \Omega_{\mathrm{s}}$, we set adiabatic BCs, which avoid heat transfer outside of the boundary. Mathematically, they result in homogeneous Neumann BCs and are expressed as

$$
k \frac{\partial}{\partial \vec{n}} T=0, \quad \text { in } \Gamma_{\mathrm{s}},
$$

where $\vec{n}$ is the outer normal vector to $\Gamma_{\mathrm{s}}$.

\subsection{Electric circuits}

In classic circuit or network analysis, the electric behaviour of the circuit is not represented by a 3D space dependent physical domain, but by a directed graph which establishes the interconnections between the different circuit components representing the devices. This allows to significantly reduce the degrees of freedom required to model the circuit. The behaviour of the elements is described with lumped element models that pose an algebraic or differential relation between the voltages across and currents through the branches, where the given device is located. The aim is to find the value of these voltages and currents.

The mathematical relation between the currents or voltages is established by means of Kirchhoff's circuit laws and depends on the topology of the graph that describes the circuit. This, together with the lumped element models, allows to set up a system of differential (algebraic) equations, that can be solved in time in order to simulate the electric behaviour of the circuit [101, Chapter 3],[18], [102].

\subsubsection{From Maxwell to circuits}

Kirchhoff's circuit laws are formed by two equalities [101] and can be deduced from the static approximation of Maxwell's equations.

\section{Kirchhoff's current law}

We start by deriving the first law, Kirchhoff's current law (KCL), and consider the static approximation of Ampère's law (2.2b)

$$
\operatorname{curl} \vec{H}=\vec{J} \text {. }
$$

Applying the divergence operator, we obtain that $\operatorname{div} \vec{J}=0$. This can be integrated over a volume $V$ and by applying Gauss's theorem we obtain

$$
\int_{V} \operatorname{div} \vec{J} \mathrm{~d} V=\int_{\partial V} \vec{J} \cdot \mathrm{d} \vec{A}=0
$$




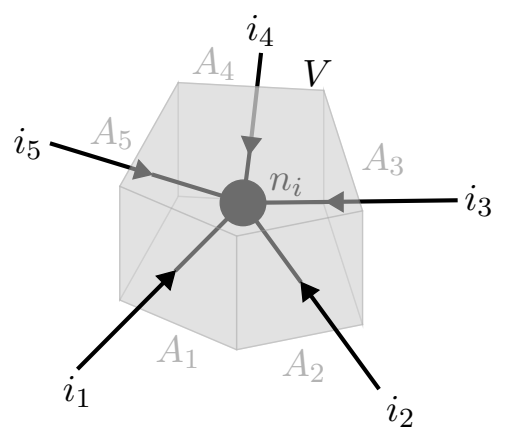

(a) Currents entering a node.

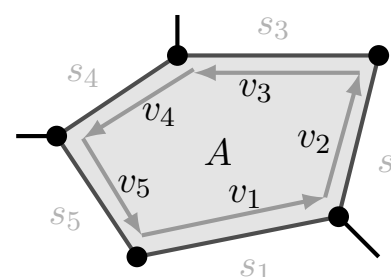

(b) Voltages around a closed loop.

Figure 2.8: Sketch of the volume around circuit node and area inside circuit loop.

Let us take $V$ now to be a volume around a node $n_{i}$ (see Figure 2.8a) and $i_{k}, k=1, \ldots, n$ the currents entering $n_{i}$. Given $A_{k}$ to be the cross-section of the $k$-th conductor with $\partial V \cap A_{k}=A_{k}$, then we have

$$
i_{k}=\int_{A_{k}} \vec{J} \cdot \mathrm{d} \vec{A}
$$

As we assume the current densities to be zero outside of the circuit's branches or conductors, we can rewrite

$$
0=\int_{\partial V} \vec{J} \cdot \mathrm{d} \vec{A}=\sum_{k=1}^{n} \int_{A_{k}} \vec{J} \cdot \mathrm{d} \vec{A}=\sum_{k=1}^{n} i_{k},
$$

which yields Kirchhoff's current law.

Kirchhoff's current law. The sum of all the currents $i_{1}, \ldots, i_{n}$ entering a node is zero, that is,

$$
\sum_{k=1}^{n} i_{k}=0
$$

\section{Kirchhoff's voltage law}

To derive the second law, Kirchhoff's voltage law (KVL) we start from the static approximation of Faraday's law (2.2a)

$$
\operatorname{curl} \vec{E}=0 \text {. }
$$

This is integrated over an area $A$ and Stoke's theorem is applied such that

$$
\int_{A} \operatorname{curl} \vec{E} \cdot \mathrm{d} \vec{A}=\int_{\partial A} \vec{E} \cdot \mathrm{d} \vec{s} .
$$

Let $A$ be the area surrounded by a loop of $n$ conductors with voltages $v_{k}, k=1, \ldots, n$ (see Figure 2.8b). Then, the voltage across the $k$-th branch or conductor is defined as

$$
v_{k}=\int_{s_{k}} \vec{E} \cdot \mathrm{d} \vec{s}
$$


where $s_{k}$ is a path across the conductor. Then, as due to the definition of the area $A$, we have $\partial A=s_{1} \cup \ldots \cup s_{n}$, Kirchhoff's voltage law is obtained as

$$
0=\int_{\partial A} \vec{E} \cdot \mathrm{d} \vec{s}=\sum_{k=1}^{n} \int_{s_{k}} \vec{E} \cdot \mathrm{d} \vec{s}=\sum_{k=1}^{n} v_{k} .
$$

Kirchhoff's voltage law. The sum of the voltages $v_{1}, \ldots, v_{n}$ around any closed loop is zero, that is,

$$
\sum_{k=1}^{n} v_{k}=0 \text {. }
$$

\section{Node potentials}

One last quantity, which is relevant when describing electric circuits, are the node potentials $e_{i}$ on the circuit nodes $n_{i}, k=i, \ldots, n$. They represent the value of the electric scalar potential $\phi$ (see Section 2.5.2) at node $n_{i}$, i.e.

$$
e_{i}=\phi_{\left.\right|_{n_{i}}} .
$$

Their relation to the voltage across a given branch of the circuit can be demonstrated by again starting from a static configuration of Faraday's law (2.46), which, as seen in Section 2.5.2, allows to define the electric scalar potential as

$$
\vec{E}=-\operatorname{grad} \phi .
$$

Let us consider the voltage $v_{i}$ between node $n_{i}$ and $n_{i+1}$, which, according to the definition of voltage is computed by means of

$$
v_{i}=\int_{s_{i}} \vec{E} \cdot \mathrm{d} \vec{s}
$$

where $s_{i}$ is a path going from $n_{i}$ to $n_{i+1}$. Given a parametrisation $\vec{r}_{i}:[a, b] \subset \mathbb{R} \rightarrow \mathbb{R}^{3}$ of the curve described by $s_{i}$, where $r_{i}(a)$ is node $i$ and $r_{i}(b)$ node $i+1$, then

$$
\begin{aligned}
v_{i} & =-\int_{s_{i}} \operatorname{grad} \phi \cdot \mathrm{d} \vec{s}=-\int_{a}^{b} \operatorname{grad} \phi(\vec{r}(s)) \cdot \vec{r}^{\prime}(s) \mathrm{d} s \\
& =-\phi(\vec{r}(b))+\phi(\vec{r}(a))=-e_{i+1}+e_{i} .
\end{aligned}
$$

\subsubsection{Lumped element models}

There are several constitutive equations that describe the behaviour of the different devices in the circuit by lumped element models. Even though also more complicated elements, such as diodes or controlled sources [101], can be used, we now only present the classical elements, that is, capacitors (C), inductors $(\mathrm{L})$, resistors (R) and time dependent voltage (V) and current (I) sources. Nonetheless, some of the more sophisticated models, that are not explained in this section are still valid elements for the circuits of the analysis in Chapter 4.

Lumped element models. We introduce the notation of $\mathbf{i}_{\star}: \mathcal{I} \rightarrow \mathbb{R}^{n_{\star}}$ and $\mathbf{v}_{\star}: \mathcal{I} \rightarrow \mathbb{R}^{n_{\star}}$ as the vector of currents through and voltages across branches containing the given element $\star \in\{\mathrm{L}, \mathrm{C}, \mathrm{R}, \mathrm{V}, \mathrm{I}\}$. Then we have that 


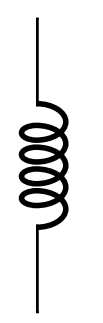

(a) Inductor

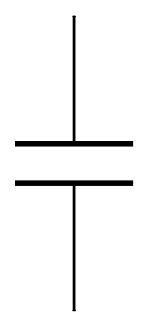

(b) Capacitor

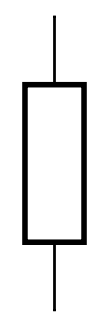

(c) Resistor

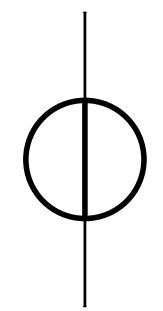

(d) Voltage source

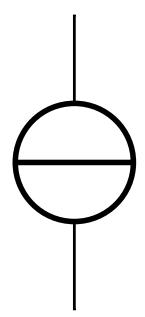

(e) Current source

Figure 2.9: Symbols for the circuit elements.

(a) inductors are described by the differential relation

$$
\mathbf{v}_{\mathrm{L}}=\frac{\mathrm{d}}{\mathrm{d} t} \phi_{\mathrm{L}}\left(\mathbf{i}_{\mathrm{L}}, t\right),
$$

with $\phi: \mathbb{R}^{n_{\mathrm{L}}} \times \mathcal{I} \rightarrow \mathbb{R}^{n_{\mathrm{L}}}$.

(b) capacitors are described by the differential relation

$$
\mathbf{i}_{\mathrm{C}}=\frac{\mathrm{d}}{\mathrm{d} t} \mathbf{q}_{\mathrm{C}}\left(\mathbf{v}_{\mathrm{C}}, t\right),
$$

with $\mathbf{q}_{\mathrm{C}}: \mathbb{R}^{n_{\mathrm{C}}} \times \mathcal{I} \rightarrow \mathbb{R}^{n_{\mathrm{C}}}$.

(c) resistors are described by the algebraic relation

$$
\mathbf{i}_{\mathrm{R}}=\mathbf{g}_{\mathrm{R}}\left(\mathbf{v}_{\mathrm{R}}, t\right),
$$

with $\mathbf{g}_{\mathrm{R}}: \mathbb{R}^{n_{\mathrm{R}}} \times \mathcal{I} \rightarrow \mathbb{R}^{n_{\mathrm{R}}}$.

(d) voltage sources are described by the algebraic relation

$$
\mathbf{v}_{\mathrm{V}}=\mathbf{v}_{\mathbf{s}}(t),
$$

with $\mathbf{v}_{\mathrm{s}}: \mathcal{I} \rightarrow \mathbb{R}^{n_{\mathrm{N}}}$.

(e) current sources are described by the algebraic relation

$$
\mathbf{i}_{\mathrm{I}}=\mathbf{i}_{\mathrm{S}}(t),
$$

with $\mathbf{i}_{\mathrm{s}}: \mathcal{I} \rightarrow \mathbb{R}^{n_{\mathrm{I}}}$.

For a mathematical discussion of the modelling behind resistors, capacitors and inductors starting from Maxwell's equations see [102]. In Figure 2.9 the different schematic symbols used to represent the circuit's elements inside the graph can be visualised. 


\subsubsection{Modified nodal analysis}

Modern electric circuit simulation is often performed with SPICE-like programs [101] (Simulation Program with Integrated Circuit Emphasis). Mathematically, they are based on the modified nodal analysis (MNA) [101], [103], which is an extension of classic nodal analysis by not only considering the node potentials as degrees of freedom of the system, but also some of the branch currents. This allows to incorporate a wider range of elements into the circuit, such as current dependent ones or inductors, and yields an easier handling of voltage sources [103]. It also keeps the sparsity of the system matrices of classic nodal analysis.

The topology of the circuits is described by an incidence matrix $\mathbf{A}^{*}$ with

$$
\left(\mathbf{A}^{*}\right)_{i j}= \begin{cases}1 & \text { if branch } j \text { leaves node } i \\ -1 & \text { if branch } j \text { enters node } i \\ 0 & \text { otherwise. }\end{cases}
$$

By choosing a reference node with potential $e_{0}=0$ called the ground node, we can define the reduced incidence matrix $\mathbf{A}$, which corresponds to the incidence matrix $\mathbf{A}^{*}$ with the deletion of the row $j_{0}$ of the reference potential.

As $\mathbf{A}^{*}$ has linearly dependent rows, for the description of the circuit's topology it is enough to use the reduced incidence matrix. We can now use the reduced incidence matrix A to enforce KCL [18] for the entire circuit as

$$
\mathbf{A} \mathbf{i}=0,
$$

where $\mathbf{i}: \mathcal{I} \rightarrow \mathbb{R}^{n_{\mathrm{i}}}$ is the vector of all the branch currents. Also, given the vector of node potentials $\mathbf{e}: \mathcal{I} \rightarrow$ $\mathbb{R}^{n_{\mathrm{e}}}$ without the ground node, the vector of voltages across all branches $\mathbf{v}: \mathcal{I} \rightarrow \mathbb{R}^{n_{\mathrm{v}}}$ can be extracted as

$$
\mathbf{A}^{\top} \mathbf{e}=\mathbf{v} .
$$

The last ingredient to set up the system of equations of the MNA are the constitutive equations for the lumped element models (2.47)-(2.51). Using KCL in (2.52), the relation between voltages and node potentials (2.53) and the constitutive equations (2.47)-(2.51), the following system of DAEs of the conventional modified nodal analysis is obtained [18]

$$
\begin{aligned}
\mathbf{A}_{\mathrm{C}} \frac{\mathrm{d}}{\mathrm{d} t} \mathbf{q}_{\mathrm{C}}\left(\mathbf{v}_{\mathrm{C}}, t\right)+\mathbf{A}_{\mathrm{R}} \mathbf{g}_{\mathrm{R}}\left(\mathbf{v}_{\mathrm{R}}, t\right)+\mathbf{A}_{\mathrm{L}} \mathbf{i}_{\mathrm{L}}+\mathbf{A}_{\mathrm{V}} \mathbf{i}_{\mathrm{V}}+\mathbf{A}_{\mathrm{I}} \mathbf{i}_{\mathrm{s}}(t) & =0, \\
\frac{\mathrm{d}}{\mathrm{d} t} \phi_{\mathrm{L}}\left(\mathbf{i}_{\mathrm{L}}, t\right)-\mathbf{A}_{\mathrm{L}}^{\top} \mathbf{e} & =0, \\
\mathbf{A}_{\mathrm{V}}^{\top} \mathbf{e}-\mathbf{v}_{\mathrm{s}} & =0,
\end{aligned}
$$

for $t \in \mathcal{I}$. Following the notation introduced in Section 2.8.2, the matrices $\mathbf{A}_{\star}$ contain the columns of the incidence matrix associated to a given element $\star \in\{\mathrm{L}, \mathrm{C}, \mathrm{R}, \mathrm{V}, \mathrm{I}\}$. The degrees of freedom are the node potentials $\mathbf{e}$ and the currents across inductors $\mathbf{i}_{\mathrm{L}}$ and voltage sources $\mathbf{i}_{\mathrm{V}}$. Given initial conditions $\mathbf{e}_{0}, \mathbf{i}_{\mathrm{L}, 0}$ and $\mathbf{i}_{\mathrm{V}, 0}$ at $t_{0}$, an initial value problem, that can then be resolved in time is obtained.

Alternatively, a second formulation can be derived by adding as degrees of freedom the charges $\mathbf{q}: \mathcal{I} \rightarrow \mathbb{R}^{n_{\mathrm{C}}}$ of capacitances and the fluxes $\phi: \mathcal{I} \rightarrow \mathbb{R}^{n_{\mathrm{L}}}$ of inductances, with $n_{\mathrm{C}}$ and $n_{\mathrm{L}}$ being the number of capacitances 
and inductances, respectively, in the circuit. Here, the system DAEs for flux-charge modified nodal analysis yields [18], [104]

$$
\begin{aligned}
& \mathbf{A}_{\mathrm{C}} \frac{\mathrm{d}}{\mathrm{d} t} \mathbf{q}+\mathbf{A}_{\mathrm{R}} \mathbf{g}_{\mathrm{R}}\left(\mathbf{v}_{\mathrm{R}}, t\right)+\mathbf{A}_{\mathrm{L}} \mathbf{i}_{\mathrm{L}}+\mathbf{A}_{\mathrm{V}} \mathbf{i}_{\mathrm{V}}+\mathbf{A}_{\mathrm{I}} \mathbf{i}_{\mathrm{s}}(t)=0, \\
& \mathbf{q}_{\mathrm{C}}\left(\mathbf{v}_{\mathrm{C}}, t\right)-\mathbf{q}=0, \\
& \phi_{\mathrm{L}}\left(\mathbf{i}_{\mathrm{L}}, t\right)-\boldsymbol{\phi}=0, \\
& \frac{\mathrm{d}}{\mathrm{d} t} \boldsymbol{\phi}-\mathbf{A}_{\mathrm{L}}^{\top} \mathbf{e}=0, \\
& \mathbf{A}_{\mathrm{V}}^{\top} \mathbf{e}-\mathbf{v}_{\mathrm{S}}=0 . \\
&
\end{aligned}
$$

Note that here, the mass matrix is always linear, that is, there are only linear terms in front of the time derivatives. For a discussion of the advantages of using flux-charge MNA (2.55) instead of conventional MNA (2.54) see [104, Chapter 1].

Some further physically sensible assumptions on topological aspects of the circuit as well as on the constitutive relations of the lumped elements have to be stated, so as to ensure existence and uniqueness of solutions of the initial value problem. We first introduce the assumption on the Jacobian of the lumped element models and deal with the structural aspects of the circuit in the following subsection.

Assumption 9 (Positive definite lumped elements [18]). The functions describing the lumped elements $\phi_{\mathrm{L}}\left(\mathbf{i}_{\mathrm{L}}, t\right)$, $\mathbf{q}_{\mathrm{C}}\left(\mathbf{v}_{\mathrm{C}}, t\right)$ and $\mathbf{g}_{\mathrm{R}}\left(\mathbf{v}_{\mathrm{R}}, t\right)$ are continuously differentiable with positive definite Jacobians

$$
\mathbf{L}\left(\mathbf{i}_{\mathrm{L}}, t\right):=\frac{\partial}{\partial \mathbf{i}_{\mathrm{L}}} \boldsymbol{\phi}_{\mathrm{L}}\left(\mathbf{i}_{\mathrm{L}}, t\right), \quad \mathbf{C}\left(\mathbf{i}_{\mathrm{C}}, t\right):=\frac{\partial}{\partial \mathbf{i}_{\mathrm{C}}} \mathbf{q}_{\mathrm{C}}\left(\mathbf{v}_{\mathrm{C}}, t\right) \quad \text { and } \quad \mathbf{G}\left(\mathbf{i}_{\mathrm{R}}, t\right):=\frac{\partial}{\partial \mathbf{i}_{\mathrm{R}}} \mathbf{g}_{\mathrm{R}}\left(\mathbf{v}_{\mathrm{R}}, t\right) .
$$

Here, $\mathbf{L}$ represents the (differential) inductance, $\mathbf{C}$ the (differential) capacitance and $\mathbf{G}$ the (differential) conductance.

\subsubsection{Circuit topology}

For the analysis of the topological properties of electric circuits, some notions of graph theory are relevant and therefore have to be introduced. Mathematically important statements about the existence and uniqueness of solution of the circuit's system, as well as its sensitivity towards perturbations are in general related to properties of the products of certain matrices. These constraints can be translated into viewable statements about topological features of the graph representing the circuit by using those concepts of graph theory.

Definition 7 (Cutsets and loops [105, Appendix A.1]). Given a connected graph $G=(V, E)$, then we define a

(a) cutset as a set of branches $E_{\mathrm{c}} \subseteq E$ such that is deletion of $G, G^{\prime}=G-E_{\mathrm{c}}$ is a disconnected graph and if any branch $e_{\mathrm{c}} \in E_{\mathrm{c}}$ is added again to $G^{\prime}$, the resulting graph is connected.

(b) loop as a subgraph $G_{1}$ of $G$, such that it is connected and every vertex $v_{1}$ in $G_{1}$ connects exactly two edges with each other. 


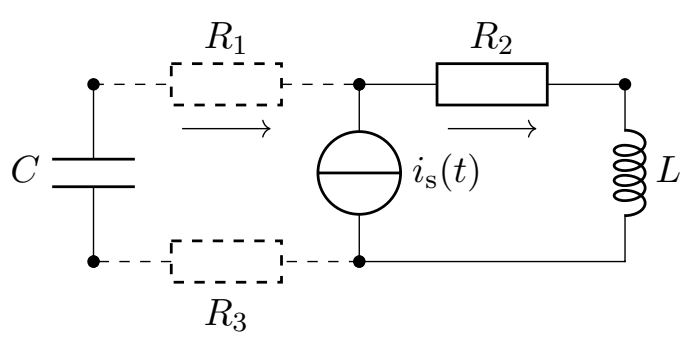

(a) Cutset

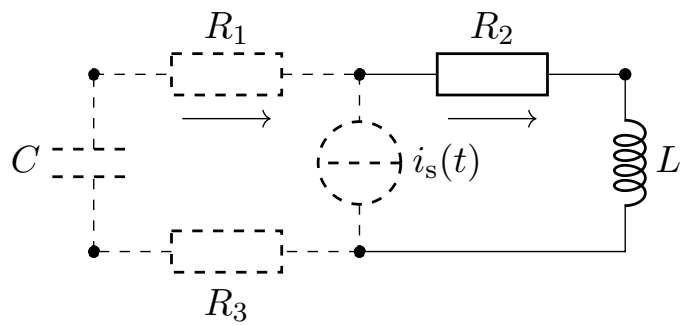

(b) Loop

Figure 2.10: Example of cutset (left) and loop (right), both highlighted in dashed.

For an example of a circuit with a cutset and a loop marked with dashed lines see Figure 2.10.

One last topological assumption on the circuit has to be stated, so as to ensure uniqueness and existence of solution. For that, we define $\mathbf{A}_{\star}^{c}$ as the matrix containing all the columns of the incidence matrix $\mathbf{A}$ associated to branches that do not contain element $\star$.

Assumption 10 (Circuit topology [18], [55]). The circuits described by MNA with (2.54) or (2.55) are assumed to fulfil the following two properties.

(a) There are no cutsets of only current sources, which mathematically can be written as

$$
\operatorname{ker}\left(\mathbf{A}_{\mathrm{I}}^{\mathrm{c}}\right)^{\top}=\{0\} .
$$

(b) There are no loops of only voltage sources, that is,

$$
\operatorname{ker} \mathbf{A}_{\mathrm{V}}=\{0\} .
$$

This assumption is required as, due to KCL, cutsets of only current sources and, due to KVL, loops of only voltage sources would lead to either no solution or infinitely many solutions of the system. 


\section{Numerical Methods and Model Analysis}

For real world application set-ups, a closed-form solution of the systems of equations that model the physical phenomena is rare. Therefore, numerical methods are used that yield an approximation of the solution. The systems of differential equations presented in the previous section are both space-dependent boundary value problems as well as time-dependent initial value problems. To approximate the solution of these two types of problems, different numerical techniques are used. This section deals with the theoretical fundamentals of these methods, as well as important concepts concerning the analysis of the systems of equations.

First, two space discretisation methods that are applied to boundary value problems (BVPs), the finite integration technique (FIT) and the finite element method (FEM), are presented. Afterwards, different time discretisation techniques for intial value problems (IVPs), as well as important theoretical fundamentals of the analysis of time discretisation methods, are shown. As most of the systems of equations considered in this work are systems of DAEs, the last part of the section introduces important concepts for their study as well as generalises the time integration techniques explained previously for DAEs.

\subsection{Space Discretisation}

In practice, the most common technique used when numerically solving time-dependent PDEs is the method of lines. In this approach, the PDE is first discretised in space so as to obtain a system of ordinary differential equations (ODEs) or DAEs, that can be further resolved in time with standard time-integration techniques.

We consider the spatial discretisation to be performed with the Ritz-Galerkin FEM with appropriate Whitney basis functions [106] or, alternatively, the FIT [107], [108]. For lowest order it can be shown, that they lead to equivalent systems, where FIT corresponds to FEM with midpoints quadrature rule [109].

In both cases the domain $\Omega$ is subdivided into $n$ elements $V_{i}, i=1, \ldots, n$ that form an oriented simplicial complex $G$. This set of elements is called the computational grid or mesh.

\subsubsection{Finite integration technique}

The finite integration technique is a spatial discretisation method of Maxwell's equations in integral form (2.1). It was proposed by Weiland in the 70s [107] and is based on the finite difference time domain method by Yee [110] but uses integral quantities as unknowns. The following section is an introduction into FIT and its structure and content follows [58]. 


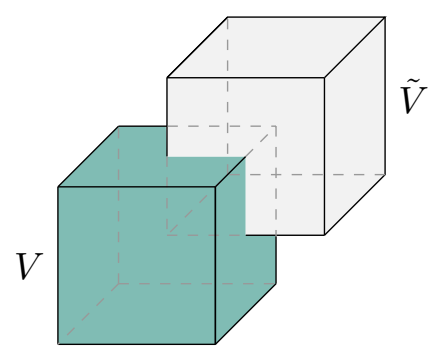

Figure 3.1: Primal and dual grid cells.

\section{Mesh}

We consider a hexahedral mesh and define the discrete degrees of freedom as integrated field quantities on its points $P_{i}$, edges $L_{i}$, facets $A_{i}$ and volumes $V_{i}$. As it can be studied by the approach of differential forms [111] and visualised in Figure 2.3, the quantities described by Maxwell's equations life on spaces that are dual to each other (see [60, Section 6.11]) and are linked by material properties (or the hodge operator in terms of differential forms). Therefore, to consistently describe Maxwell's equations, apart from the mesh $G$, where primal quantities are defined, a second dual one $\widetilde{G}$ is required for dual fields and potentials.

Whereas in FEM with appropriate basis functions this dual mesh is only constructed implicitly [64], in FIT, it needs to be defined explicitly and, in the simplest case, is built by taking the centre of the volumes in $G$ as the dual mesh's points $\widetilde{P}_{i}$ (see Figure 3.1). Dual edges $\widetilde{L}_{i}$, facets $\widetilde{A}_{i}$ and volumes $\widetilde{V}_{i}$ can then be constructed and a dual mesh $\widetilde{G}$ is obtained. The primal $G$ and dual $\widetilde{G}$ meshes, do not necessarily need to be orthogonal to each other. However, we assume them to be like this as, in this case, the system matrices acquire convenient properties [112].

\section{Maxwell's grid equations}

The integral form of Maxwell's equations (2.1) can be exactly described on the primal and dual mesh by the semidiscrete Maxwell's grid equations [107], [112]

$$
\mathbf{C} \widehat{\mathbf{e}}=-\frac{\mathrm{d}}{\mathrm{d} t} \widehat{\mathbf{b}} \quad \widetilde{\mathbf{S}} \widehat{\mathbf{d}}=\mathbf{q} \quad \widetilde{\mathbf{C}} \widehat{\mathbf{h}}=\frac{\mathrm{d}}{\mathrm{d} t} \widehat{\mathbf{d}}+\widehat{\mathbf{j}} \quad \mathbf{S} \widehat{\mathbf{b}}=0 .
$$

Here, the discrete curl operators $\mathbf{C}, \widetilde{\mathbf{C}} \in\{-1,0,1\}^{N_{\text {dof }} \times N_{\text {dof }}}$ and the discrete divergence operators $\mathbf{S}, \widetilde{\mathbf{S}} \in$ $\{-1,0,1\}^{N \times N_{\text {dof }}}$ are defined on the primal and dual mesh, respectively, with $N_{\text {dof }}$ denoting the number of primal edges and $N$ the number of primal points. The field quantities $\widehat{\mathbf{e}}, \widehat{\mathbf{b}}, \widehat{\mathbf{d}}, \widehat{\mathbf{h}}, \widehat{\mathbf{j}}: \mathcal{I} \rightarrow \mathbb{R}^{N_{\text {dof }}}$ and $\mathbf{q}: \mathcal{I} \rightarrow \mathbb{R}^{N}$ are the semidiscrete integrated degrees of freedom, such that

$$
\widehat{\mathbf{e}}_{i}:=\int_{L_{i}} \vec{E} \cdot \mathrm{d} \vec{s}, \quad \quad \widehat{\mathbf{b}}_{i}:=\int_{A_{i}} \vec{B} \cdot \mathrm{d} \vec{A}
$$

and

$$
\widehat{\mathbf{d}}_{i}:=\int_{\widetilde{A}_{i}} \vec{D} \cdot \mathrm{d} \vec{A}, \quad \widehat{\mathbf{h}}_{i}:=\int_{\widetilde{L}_{i}} \vec{H} \cdot \mathrm{d} \vec{s}, \quad \widehat{\mathbf{j}}_{i}:=\int_{\widetilde{A}_{i}} \vec{J} \cdot \mathrm{d} \vec{A}, \quad \mathbf{q}_{j}=\int_{\widetilde{V}_{j}} \rho \mathrm{d} V
$$

for $i=1, \ldots, N_{\text {dof }}$ and $j=1 \ldots N$. 
Analogous to the continuous case, potentials can be introduced (see Section 2.5) as integrated quantities on the primal and dual mesh, respectively. They are defined as $\widehat{\mathbf{a}}, \widehat{\mathrm{t}}: \mathcal{I} \rightarrow \mathbb{R}^{N_{\text {dof }}}$ and $\phi, \Psi: \mathcal{I} \rightarrow \mathbb{R}^{N}$

$$
\widehat{\mathbf{a}}_{i}:=\int_{L_{i}} \vec{A} \cdot \mathrm{d} \vec{s}, \quad \widehat{\mathbf{t}}_{i}:=\int_{\widetilde{L}_{i}} \vec{T} \cdot \mathrm{d} \vec{s}, \quad \quad \boldsymbol{\Psi}_{j}:=\psi_{\widetilde{P}_{j}}, \quad \quad \phi_{j}:=\phi_{\left.\right|_{P_{j}}},
$$

for $i=1, \ldots, N_{\text {dof }}$ and $j=1 \ldots N$.

\section{Material matrices}

As for now, Maxwell's grid equations (3.1) with the integrated field quantities as degrees of freedom do not include any approximation error. However, a link between dual quantities has to be established by means of material matrices [107], [112]

$$
\widehat{\widehat{d}}=\mathbf{M}_{\varepsilon} \widehat{\mathbf{e}} \quad \widehat{\mathrm{j}}_{\mathrm{c}}=\mathbf{M}_{\sigma} \widehat{\mathbf{e}} \quad \widehat{\mathbf{h}}=\mathbf{M}_{\nu} \widehat{\mathrm{b}} .
$$

The matrices $\mathbf{M}_{\varepsilon}=\mathbf{M}_{\varepsilon}(\widehat{\mathbf{e}}), \mathbf{M}_{\sigma}=\mathbf{M}_{\sigma}(\widehat{\mathbf{e}})$ and $\mathbf{M}_{\nu}=\mathbf{M}_{\nu}(\widehat{\mathrm{b}})$ represent the permittivity, conductivity and reluctivity and $\widehat{\mathbf{j}}_{\mathrm{c}}:=\mathcal{I} \rightarrow \mathbb{R}^{N_{\text {dof }}}$ the integrated conduction current density. Again, the inverse of the previous relations can be described with the resistivity and permeability matrices $\mathbf{M}_{\rho}=\mathbf{M}_{\rho}\left(\widehat{\mathbf{j}}_{\mathrm{c}}\right)$ and $\mathbf{M}_{\mu}=\mathbf{M}_{\mu}(\widehat{\mathbf{h}})$, such that

$$
\widehat{\mathbf{e}}=\mathbf{M}_{\rho} \widehat{\hat{\mathbf{j}}}_{\mathrm{c}} \quad \text { and } \quad \widehat{\mathbf{b}}=\mathbf{M}_{\mu} \widehat{\mathbf{h}} .
$$

These relations introduce now a discretisation error, due to the element-wise homogenization of the materials and of the field quantities [112].

Remark 8. Given the specific numbering scheme for the degrees of freedom commonly used in FIT and with orthogonal primal and dual meshes, the material matrices are diagonal for isotropic materials [112].

\section{Matrix construction}

Following [58], we will illustrate the construction of the FIT operator and material matrices with two examples, the curl operator in Faraday's law (2.1a) and the conductivity in (2.3). To define the discrete curl operator matrix, we consider Faraday's law in integral form on a primal facet $A_{\omega}$ of the mesh $G$

$$
\int_{\partial A_{\omega}} \vec{E} \cdot \mathrm{d} \vec{s}=-\int_{A_{\omega}} \frac{\partial}{\partial t} \vec{B} \cdot \mathrm{d} \vec{A}
$$

For its associated primal edges $L_{\omega, 1}, L_{\omega, 2}, L_{\omega, 3}, L_{\omega, 4}$ given in Figure 3.2 such that

$$
\partial A_{\omega}=L_{\omega, 1} \cup L_{\omega, 2} \cup-L_{\omega, 3} \cup-L_{\omega, 4}
$$

we have

$$
\int_{\partial A_{\omega}} \vec{E} \cdot \mathrm{d} \vec{s}=\int_{L_{\omega, 1}} \vec{E} \cdot \mathrm{d} \vec{s}+\int_{L_{\omega, 2}} \vec{E} \cdot \mathrm{d} \vec{s}+\int_{-L_{\omega, 3}} \vec{E} \cdot \mathrm{d} \vec{s}+\int_{-L_{\omega, 4}} \vec{E} \cdot \mathrm{d} \vec{s} \cdot
$$

The signs of the integrals along the edges depend on the orientation of the different geometrical elements in the mesh. For the orientation given in Figure 3.2, which is the typical orientation obtained from the special 


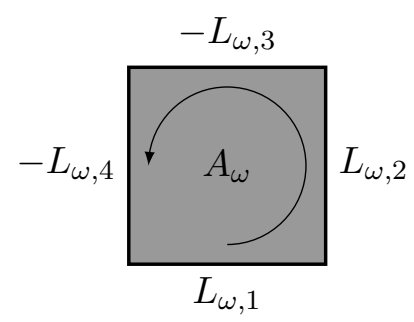

Figure 3.2: Orientation of primal facet $A_{\omega}$ and boundary edges $L_{\omega, i}$.

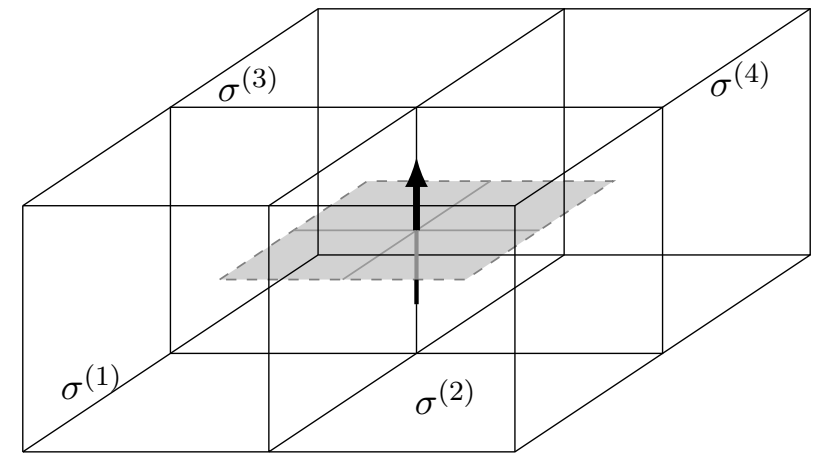

Figure 3.3: Sketch of dual facet $\widetilde{A}_{\omega}$ with its normal vector and surrounding primal volumes [58].

numbering scheme used in FIT (see [113, Appendix A.1],[114]), the signs are obtained as given above. Due to the definition of the integral field quantities (3.2), we have

$$
\widehat{\mathbf{e}}_{\omega, 1}+\widehat{\mathbf{e}}_{\omega, 2}-\widehat{\mathbf{e}}_{\omega, 3}-\widehat{\mathbf{e}}_{\omega, 4}=-\frac{\mathrm{d}}{\mathrm{d} t} \widehat{\mathbf{b}}_{\omega} .
$$

Performing this for all facets of the primal grid $G$ yields the semidiscrete Faraday law (3.1)

$$
\mathbf{C e}=\frac{\mathrm{d}}{\mathrm{d} t} \widehat{\mathbf{b}}
$$

with the discrete primal curl matrix

$$
\mathbf{C}=\left[\begin{array}{cccccccc} 
& \multicolumn{1}{c}{} & & \vdots & & & \\
\cdots & 1 & \cdots & -1 & \cdots & -1 & 1 & \cdots \\
& & & & \vdots & & &
\end{array}\right] .
$$

Analogously, this can be done for the rest of the integral Maxwell's equations (2.1), to obtain the discrete primal and dual divergence matrices and the dual curl matrix. For a detailed description of the operator matrices see [113, Appendix A.1],[114], [115].

For simplicity of notation in the material matrix exemplification we assume a field-independent conductivity that is isotropic and constant on each primal volume $V_{i}$. Given a dual facet $\widetilde{A}_{\omega}$ and its associated primal edge $L_{\omega, \mathrm{n}}$ (see Figure 3.3), the integrated current density is

$$
\widehat{\mathbf{j}}_{\omega}=\int_{\widetilde{A}_{\omega}} \vec{J} \cdot \mathrm{d} \vec{A}=\sum_{i=1}^{4} \int_{\widetilde{A}_{\omega}^{(i)}} \sigma^{(i)} \vec{E} \cdot \mathrm{d} \vec{A},
$$


with $\widetilde{A}_{\omega}^{(i)}=\widetilde{A}_{\omega} \cap V_{\omega, i}$ and $V_{\omega, i}$ being the $i$-th primal volume surrounding $\widetilde{A}_{\omega}$. For $\vec{n}_{\omega}$ the normal vector to $\widetilde{A}_{\omega}$ and using the normal component of the electric field strength $\vec{E} \cdot \vec{n}_{\omega}=E_{\omega, \mathrm{n}}$, we can exploit to the tangential continuity of $\vec{E}$ to approximate the previous integral as

$$
\begin{aligned}
\sum_{i=1}^{4} \int_{\widetilde{A}_{\omega}^{(i)}} \sigma^{(i)} \vec{E} \cdot \mathrm{d} \vec{A} & =\sum_{i=1}^{4} \int_{\widetilde{A}_{\omega}^{(i)}} \sigma^{(i)} E_{\omega, \mathrm{n}} \mathrm{d} A \\
& \approx \sum_{i=1}^{4} \sigma^{(i)} E_{\omega, \mathrm{n}}\left|\widetilde{A}_{\omega}^{(i)}\right|
\end{aligned}
$$

where $|\cdot|$ denotes the length, area or volume of the corresponding edge, facet or volume. Approximating the integrated electric field strength by

$$
\widehat{\mathbf{e}}_{\omega, \mathrm{n}}=\int_{L_{\omega, \mathrm{n}}} \vec{E} \cdot \mathrm{d} \vec{s} \approx E_{\omega, \mathrm{n}}\left|L_{\omega, \mathrm{n}}\right|
$$

the relation

$$
\widehat{\mathbf{j}}_{\omega} \approx \sum_{i=1}^{4} \sigma^{(i)} E_{\omega, \mathrm{n}}\left|\widetilde{A}_{\omega}^{(i)}\right| \approx \sum_{i=1}^{4} \sigma^{(i)} \frac{\left|\widetilde{A}_{\omega}^{(i)}\right|}{\left|L_{\omega, \mathrm{n}}\right|} \widehat{\mathbf{e}}_{\omega, \mathrm{n}}
$$

is obtained. With this procedure a conductivity matrix can be constructed, whose entries are the averaged conductivities

$$
\left(\mathbf{M}_{\sigma}\right)_{i, j}=\sum_{k=1}^{4} \sigma^{(k)} \frac{\left|\widetilde{A}_{i}^{(k)}\right|}{\left|L_{j}\right|}
$$

whenever $L_{j}$ is the associated primal edge to $\widetilde{A}_{i}$.

The rest of the material matrices are obtained with an analogous averaging procedure. For a description of other material matries see [113, Appendix A.1],[114], [115].

Remark 9. An analogous process can be followed to discretise the heat equation of section 2.7.2 with FIT. Here, the temperature $\mathbf{T}_{i}$ is defined on the primal points $P_{i}$. For further details on the finite integration technique applied to the heat equation see [115], [116].

\subsubsection{Finite element method}

The finite element method is another numerical method for the discretisation of partial differential equations. It is based on the approximation of the PDE's weak formulation. Therefore, in contrast to FIT, the finite element method starts form the weak version of the differential form of Maxwell's equations to estimate its solution. The theory presented in this section is based on [64], [117].

\section{Basis functions}

In order to ensure that the solution fulfils some properties that are required and presented later, first some necessary theoretical definitions are introduced. 
Definition 8 (Hilbert spaces). Let $\Omega$ be a domain fulfilling Assumption 1, then we introduce the following Hilbert spaces

$$
\begin{aligned}
\mathrm{H}^{1}(\Omega)=\mathrm{H}(\operatorname{grad} ; \Omega) & :=\left\{u \in \mathbf{L}^{2}(\Omega) ; \operatorname{grad} u \in \mathbf{L}^{2}(\Omega)\right\} \\
\mathrm{H}(\operatorname{curl} ; \Omega) & :=\left\{\nu \in \mathbf{L}^{2}(\Omega) ; \operatorname{curl} \nu \in \mathbf{L}^{2}(\Omega)\right\} \\
\mathrm{H}(\operatorname{div} ; \Omega) & :=\left\{\omega \in \mathbf{L}^{2}(\Omega) ; \operatorname{div} \omega \in \mathbf{L}^{2}(\Omega)\right\}
\end{aligned}
$$

where $\mathbf{L}^{2}(\Omega)$ is the standard Hilbert space of square integrable functions and the operators div, grad and curl are to be read in a weak sense.

These spaces, together with the operators div , grad and curl form the de Rham cohomology [64]

$$
\mathbb{R} \longrightarrow \mathrm{H}^{1}(\Omega) \stackrel{\text { grad }}{\longrightarrow} \mathrm{H}(\operatorname{curl} ; \Omega) \stackrel{\text { curl }}{\longrightarrow} \mathrm{H}(\operatorname{div} ; \Omega) \stackrel{\text { div }}{\longrightarrow} \mathbf{L}^{2}(\Omega) \longrightarrow 0 \text {. }
$$

This sequence is, for domains described in Assumption 1, exact and closed [64]. For a given mesh $G$ that, analogous to the procedure taken in FIT, discretises the domain into volumes (usually tetaedra) $V_{i}$, and its corresponding facets $A_{i}$, edges $L_{i}$ and points $P_{i}$, we consider $n_{\mathrm{V}}$ volume $s_{i}, n_{\mathrm{A}}$ face $\vec{\omega}_{i}, n_{\mathrm{L}}$ edge $\vec{\nu}_{i}$ and $n_{\mathrm{P}}$ node $u_{i}$ basis functions, respectively, such that the discrete spaces they span inherit the properties of their continuous counterpart and thus also yield an exact de Rham sequence (e.g. Whitney elements [64]). Their discrete, exact and closed sequence is

$$
\begin{array}{rcc}
\mathbb{R} \longrightarrow U_{h} \stackrel{\text { grad }}{\longrightarrow} V_{h} \stackrel{\text { curl }}{\longrightarrow} W_{h} \stackrel{\text { div }}{\longrightarrow} S_{h} \longrightarrow \mathbb{R}, \\
u_{i} \quad \vec{\nu}_{i} & \vec{\omega}_{i} & s_{i}
\end{array}
$$

with the spaces defined as

$$
\begin{aligned}
U_{h} & :=\left\{f \in \mathbf{L}^{2}(\Omega) ; f=\sum_{i=1}^{n_{\mathrm{P}}} \alpha_{i} u_{i}, \alpha_{i} \in \mathbb{R}\right\} \subseteq \mathrm{H}^{1}(\Omega), \\
V_{h} & :=\left\{f \in \mathbf{L}^{2}(\Omega) ; f=\sum_{i=1}^{n_{\mathrm{L}}} \alpha_{i} \vec{\nu}_{i}, \alpha_{i} \in \mathbb{R}\right\} \subseteq \mathrm{H}(\operatorname{curl} ; \Omega), \\
W_{h} & =\left\{f \in \mathbf{L}^{2}(\Omega) ; f=\sum_{i=1}^{n_{\mathrm{A}}} \alpha_{i} \vec{\omega}_{i}, \alpha_{i} \in \mathbb{R}\right\} \subseteq \mathrm{H}(\operatorname{div} ; \Omega), \\
S_{h} & :=\left\{f \in \mathbf{L}^{2}(\Omega) ; f=\sum_{i=1}^{n_{\mathrm{V}}} \alpha_{i} s_{i}, \alpha_{i} \in \mathbb{R}\right\} \subseteq \mathbf{L}^{2}(\Omega)
\end{aligned}
$$

Remark 10. In practice, the continuous and discrete function spaces are defined such that they contain functions fulfilling the boundary conditions presented in section 2.3. For the case where homogeneous Dirichlet boundary conditions are set, the common notation is the usage of a subscript and superscript 0 for the continuous and discrete spaces, respectively.

\section{Weak formulation}

We illustrate the construction of the finite element matrices for the case of the magnetoquasistatic curl-curl PDE with the $\vec{A} *$ formulation (2.20). The weak formulation is expressed as find $\vec{A} \in \mathrm{H}$ (curl; $\Omega$ ) such that for all $\mathrm{H}$ (curl)-conforming basis functions $\vec{\nu} \in \mathrm{H}(\operatorname{curl} ; \Omega)$ [117],

$$
\int_{\Omega} \boldsymbol{\sigma} \frac{\partial}{\partial t} \vec{A} \cdot \vec{\nu} \mathrm{d} \Omega+\int_{\Omega} \operatorname{curl} \boldsymbol{\nu} \operatorname{curl} \vec{A} \cdot \vec{\nu} \mathrm{d} \Omega=\int_{\Omega} \overrightarrow{J_{\mathrm{s}}} \cdot \vec{\nu} \mathrm{d} \Omega .
$$


Applying Gauss's theorem, we obtain

$$
\int_{\Omega} \boldsymbol{\sigma} \frac{\partial}{\partial t} \vec{A} \cdot \vec{\nu} \mathrm{d} \Omega+\int_{\Omega} \boldsymbol{\nu} \operatorname{curl} \vec{A} \cdot \operatorname{curl} \vec{\nu} \mathrm{d} \Omega+\int_{\Gamma}(\boldsymbol{\nu} \operatorname{curl} \vec{A} \times \vec{\nu}) \cdot \mathrm{d} \vec{\Gamma}=\int_{\Omega} \overrightarrow{J_{\mathrm{s}}} \cdot \vec{\nu} \mathrm{d} \Omega .
$$

Considering both a magnetic vector potential $\vec{A}$ and test functions $\vec{\nu}$ in $\mathrm{H}_{\Gamma}$ (curl; $\Omega$ ), that is, $\mathrm{H}$ (curl)-conforming functions fulfilling the homogeneous Dirichlet and Neumann boundary conditions of (2.21), then

$$
\begin{aligned}
\int_{\Gamma}(\boldsymbol{\nu} \operatorname{curl} \vec{A} \times \vec{\nu}) \cdot \mathrm{d} \vec{\Gamma} & =\int_{\Gamma_{\mathrm{ebc}}}(\boldsymbol{\nu} \operatorname{curl} \vec{A} \times \vec{\nu}) \cdot \mathrm{d} \vec{\Gamma}+\int_{\Gamma_{\mathrm{mbc}}}(\boldsymbol{\nu} \operatorname{curl} \vec{A} \times \vec{\nu}) \cdot \mathrm{d} \vec{\Gamma} \\
& =\int_{\Gamma_{\mathrm{ebc}}}(\vec{\nu} \times \vec{n}) \cdot \boldsymbol{\nu} \operatorname{curl} \vec{A} \mathrm{~d} \Gamma+\int_{\Gamma_{\mathrm{mbc}}}(\vec{n} \times \boldsymbol{\nu} \operatorname{curl} \vec{A}) \cdot \vec{\nu} \mathrm{d} \Gamma \\
& =0,
\end{aligned}
$$

with $\mathrm{d} \vec{\Gamma}=\vec{n} \mathrm{~d} \Gamma$ and $\vec{n}$ the outer normal vector to $\Gamma$. In the case of the Ritz-Galerkin FEM, the magnetic vector potential is approximated by the test functions defined on the mesh as

$$
\vec{A} \approx \sum_{i=1}^{n_{\mathrm{L}}} \mathbf{a}_{i}(t) \vec{\nu}_{i}
$$

This leads to a system of equations by testing the weak formulation with all $\vec{\nu}_{i} \in V_{h}^{\Gamma}$ (the discrete counterpart of $\mathrm{H}_{\Gamma}(\operatorname{curl} ; \Omega)$ )

$$
\mathbf{M}_{\sigma}^{\mathrm{FE}} \frac{\mathrm{d}}{\mathrm{d} t} \mathbf{a}+\mathbf{K}_{\nu}^{\mathrm{FE}} \mathbf{a}=\mathbf{J}_{\mathrm{s}}^{\mathrm{FE}}
$$

with the system matrices defined as

$$
\left(\mathbf{M}_{\sigma}^{\mathrm{FE}}\right)_{i, j}=\int_{\Omega} \sigma \vec{\nu}_{i} \cdot \vec{\nu}_{j} \mathrm{~d} \Omega, \quad\left(\mathbf{K}_{\nu}^{\mathrm{FE}}\right)_{i, j}=\int_{\Omega} \boldsymbol{\nu}\left(\operatorname{curl} \vec{\nu}_{i}\right) \cdot\left(\operatorname{curl} \vec{\nu}_{j}\right) \mathrm{d} \Omega \quad \text { and } \quad\left(\mathbf{J}_{\mathrm{s}}^{\mathrm{FE}}\right)_{i}=\int_{\Omega} \vec{J}_{\mathrm{s}} \cdot \vec{\nu}_{i} \mathrm{~d} \Omega
$$

and the degrees of freedom $(\mathbf{a})_{i}:=a_{i}(t)$. As the basis functions are chosen to fulfil the closed and exact de Rham sequence, we know that curl $\vec{\nu}_{i}$ lives in the discrete counterpart of $\mathrm{H}_{\Gamma}(\operatorname{div} ; \Omega)$, that is, $W_{h}^{\Gamma}$. Therefore, for the corresponding $\mathrm{H}(\mathrm{div})$-conforming basis functions $\vec{\omega}_{j}, j=1, \ldots, n_{\mathrm{A}}$, one can write

$$
\operatorname{curl} \vec{\nu}_{i}=\sum_{j=1}^{n_{\mathrm{A}}} c_{i, j} \vec{\omega}_{j} .
$$

Thus, the FIT factorisation of matrices which separates operator and material matrices (see Section 3.1.1) can also be obtained in FEM and the curl-curl matrix is rewritten as $\mathbf{K}_{\nu}^{\mathrm{FE}}=\widetilde{\mathbf{C}}^{\mathrm{FE}} \mathbf{M}_{\nu}^{\mathrm{FE}} \mathbf{C}^{\mathrm{FE}}$, with

$$
\left(\mathbf{M}_{\nu}^{\mathrm{FE}}\right)_{i, j}=\int_{\Omega} \boldsymbol{\nu} \vec{\omega}_{i} \cdot \vec{\omega}_{j} \mathrm{~d} \Omega, \quad\left(\mathbf{C}^{\mathrm{FE}}\right)_{i, j}=c_{j, i} \quad \text { and } \quad\left(\widetilde{\mathbf{C}}^{\mathrm{FE}}\right)_{i, j}=c_{i, j} .
$$

Analogously, the rest of the PDEs obtained from the different formulations in Section 2.5 can be discretised and factorised, which lead to the rest of the material matrices $\mathbf{M}_{\varepsilon}^{\mathrm{FE}}(\mathbf{e}), \mathbf{M}_{\sigma}^{\mathrm{FE}}(\mathbf{e}), \mathbf{M}_{\nu}^{\mathrm{FE}}(\mathbf{b}), \mathbf{M}_{\rho}^{\mathrm{FE}}\left(\mathbf{j}_{\mathrm{c}}\right)$ and $\mathbf{M}_{\mu}^{\mathrm{FE}}(\mathbf{h})$, and the operator matrices $\mathbf{G}^{\mathrm{FE}}, \widetilde{\mathbf{G}}^{\mathrm{FE}}, \mathbf{S}^{\mathrm{FE}}, \widetilde{\mathbf{S}}^{\mathrm{FE}}, \mathbf{C}^{\mathrm{FE}}$ and $\widetilde{\mathbf{C}}^{\mathrm{FE}}$ for the discrete (primal and dual) gradient, divergence and curl operators, respectively.

The field quantities are approximated by

$$
\vec{E} \approx \sum_{i=1}^{n_{\mathrm{L}}} \mathbf{e}_{i}(t) \vec{\nu}_{i}, \quad \vec{B} \approx \sum_{i=1}^{n_{\mathrm{A}}} \mathbf{b}_{i}(t) \vec{\omega}_{i}
$$


and

$$
\vec{D} \approx \sum_{i=1}^{n_{\mathrm{A}}} \mathbf{d}_{i}(t) \vec{\omega}_{i}, \quad \vec{H} \approx \sum_{i=1}^{n_{\mathrm{L}}} \mathbf{h}_{i}(t) \vec{\nu}_{i}, \quad \vec{J} \approx \sum_{i=1}^{n_{\mathrm{A}}} \mathbf{j}_{i}(t) \vec{\omega}_{i}, \quad \rho \approx \sum_{i=1}^{n_{\mathrm{V}}} \mathbf{q}_{i}(t) s_{i},
$$

where $s_{i}, i=1, \ldots, n$ are $\mathrm{L}_{\Gamma}^{2}$-conforming basis functions spanning $S_{h}^{\Gamma}$. The potentials defined in Section 2.5 are approximated by

$$
\vec{A} \approx \sum_{i=1}^{n_{\mathrm{L}}} \mathbf{a}_{i}(t) \vec{\nu}_{i}, \quad \vec{T} \approx \sum_{i=1}^{n_{\mathrm{L}}} \mathbf{t}_{i}(t) \vec{\nu}_{i}, \quad \psi \approx \sum_{i=1}^{n_{\mathrm{P}}} \boldsymbol{\Psi}_{i}(t) u_{i}, \quad \phi \approx \sum_{i=1}^{n_{\mathrm{P}}} \phi_{i}(t) u_{i},
$$

with $u_{i}$, for $i=1, \ldots, \widetilde{n}$, being $\mathrm{H}^{1}$-conforming basis functions.

\section{Heat equation}

The temperature $T$ in the heat equation (2.41) requires only $\mathrm{H}^{1}$-conforming basis functions and is approximated by

$$
T \approx \sum_{i=1}^{n_{\mathrm{P}}} \boldsymbol{T}_{i}(t) u_{i}
$$

The material matrices $\mathbf{M}_{\mathrm{k}}$ and $\mathbf{M}_{\rho, \mathrm{C}}$ are obtained for the thermal conductivity and the product of the mass density and the heat capacity, respectively.

\subsubsection{Matrix properties}

For simplicity of notation and, as we consider systems of equations arising from both FEM and FIT discretisations, from now on we will overload the symbols introduced in Sections 3.1.1 and 3.1.2 and use always the symbols from (3.1), (3.5) and (3.6) for the discrete operator and material matrices, independently of the discretisation method that has been used (FEM and FIT). The degrees of freedom, however, are denoted, like in the FE case, in bold. That is, we leave out the 'bows' used within the FIT framework, see (3.2)-(3.4).

We present now some properties of both the operator as well as the material matrices, that are inherited from the properties of the continuous operators and materials they represent. To be able to obtain uniqueness of solution in the discrete systems, we need the following assumption.

Assumption 11 (Boundary conditions). For FIT, the degrees of freedom are projected to an appropriate subspace, which imposes homogeneous Dirichlet boundary conditions. In the FE case, we assume the chosen basis functions fulfil the given homogeneous Dirichlet boundary conditions. This yields projected operator matrices $\mathbf{G}, \widetilde{\mathbf{G}}, \mathbf{S}, \widetilde{\mathbf{S}}$, $\mathbf{C}$ and $\widetilde{\mathbf{C}}$, with

$$
\operatorname{ker} \widetilde{\mathbf{S}}^{\top}=0 \quad \text { and } \quad \operatorname{ker} \mathbf{S}^{\top}=0 .
$$

Lemma 1 (Topological matrices). The discrete operator matrices [112] fulfil that 
(a) analogous to the continuous case, the divergence of the curl and the curl of the gradient vanish

$$
\mathbf{S C}=0, \widetilde{\mathbf{S}} \widetilde{\mathbf{C}}=0 \text { and } \mathbf{C G}=0, \widetilde{\mathbf{C}} \widetilde{\mathbf{G}}=0 .
$$

(b) considering a contractible domain as described in Assumption 1 and fulfilling Assumption 11,

$$
\operatorname{dim} \operatorname{ker}(\widetilde{\mathbf{S}})=\operatorname{rank}(\mathbf{C}) .
$$

(c) the curl and dual curl are linked with

$$
\widetilde{\mathbf{C}}=\mathbf{C}^{\top} \text {. }
$$

(d) the primal (dual) gradient and dual (primal) divergence are related by

$$
\mathbf{G}=-\widetilde{\mathbf{S}}^{\top} \text { and } \quad \widetilde{\mathbf{G}}=-\mathbf{S}^{\top} \text {. }
$$

For the case of the topological FIT matrices, these properties are well known, see e.g. [112], [118] or [119, Section 3.2.4] for the reduced operator matrices obtained after Assumption 11.

In the case of FEM, Lemma 1.a) follows immediately from the fact that the spaces spanned by the basis functions fulfil the exact de Rham sequence. Lemma 1.c) is an immediate result of using a Ritz-Galerkin weak formulation and can be immediately seen in (3.8). The last property follows analogously.

Lemma 2 (Material Matrices). The material matrices $\mathbf{M}_{\xi}$ are symmetric for all material properties

$$
\xi=\left\{\varepsilon, \boldsymbol{\sigma}, \boldsymbol{\nu}, \boldsymbol{\rho}, \boldsymbol{\mu}, \nu \tau_{\mathrm{eq}}\right\} .
$$

If Assumption 3 and 8 holds, then the matrices $\mathbf{M}_{\varepsilon}, \mathbf{M}_{\nu}, \mathbf{M}_{\mu}$ are positive definite whereas $\mathbf{M}_{\sigma}, \mathbf{M}_{\rho}, \mathbf{M}_{\nu, \tau_{\mathrm{eq}}}$ are only positive semidefinite.

\subsection{Time Discretisation}

The circuit systems as well as the semidiscrete systems of Section 2, are time-dependent systems of differential equations. In order to solve them on a time window $\mathcal{I}$, initial conditions are set at initial time $t_{0}$, and numerical time integration techniques are applied to them. The theoretical results presented in this section mainly follow [101, Chapter VI],[120], [121].

In general, the time-dependent systems we consider can be either be systems of ODEs or DAEs. Nonetheless, as the classic theory regarding time integration techniques is originally formulated for ODEs, we will start to consider IVPs arising from ODEs, that is,

$$
\begin{aligned}
\mathbf{x}^{\prime} & =\mathbf{f}(\mathbf{x}, t), \\
\mathbf{x}\left(t_{0}\right) & =\mathbf{x}_{0},
\end{aligned}
$$


for $t \in \mathcal{I}=\left[t_{0}, t_{\text {end }}\right)$ and with $\mathbf{x}: \mathcal{I} \rightarrow \mathbb{R}^{n_{\text {dof }}}$ being the degrees of freedom, $n_{\text {dof }}$ the number of degrees of freedom and $\mathbf{x}_{0}$ the ICs. The symbol $\mathrm{x}^{\prime}$ denotes the time derivated degrees of freedom $\mathbf{x}$.

Classic ODE theory states that, provided the function $\mathbf{f}(\mathbf{x}, t)$ is Lipschitz continuous with respect to $\mathbf{x}$ for all $t \in$ $\mathcal{I}$, existence and uniqueness of solution of the initial value problem (3.16) can be ensured [122].

To calculate the time evolution of the IVP (3.16), we start by dividing the time interval $\mathcal{I}$ into $n_{\mathrm{t}}+1$ subintervals $\left[t_{i}, t_{i+1}\right), i=0, \ldots, n_{\mathrm{t}}$ of size $h_{i+1}=t_{i+1}-t_{i}$ and then numerically approximate with a time integration method the solution at the given points by $\left\{\mathbf{x}_{0}, \ldots, \mathbf{x}_{n_{\mathrm{t}}}\right\}$.

Remark 11. Note that the time step size $h_{i+1}$ can be adapted to the local requirement of the differential equation at time $t_{i+1}$, as it can vary. Adaptive time step sizes are important in practical applications to save computational cost. Step size control methods allow reducing or augmenting the time step size and keep control over the obtained error through error estimates. For more details about time step size control methods see [120]. This work, however, does not deal with adaptive time step sizes in detail and thus uses constant time step sizes in most of the cases.

\subsubsection{Theoretical fundamentals}

In the study of time integration techniques, two main theoretical concepts arise for the analysis and comparison of different methods, the local truncation error (LTE) and the stability. In the following section we will introduce both concepts and use them to describe the two methods, that are presented. Let us consider a general, linear (possibly) multistep method [120, Chapter 3]

$$
\sum_{i=0}^{k} \alpha_{i} \mathbf{x}_{n+i}=h \sum_{i=0}^{k} \beta_{i} \mathbf{f}\left(\mathbf{x}_{n+i}, t_{n+i}\right),
$$

where $h$ is the (constant) time step size and $\alpha_{i}$ and $\beta_{i}$ are real coefficients such that $\alpha_{k} \neq 0$ and $\left|\alpha_{0}\right|+\left|\beta_{0}\right|>0$. For given previous solutions $\mathbf{x}_{n}, \ldots, \mathbf{x}_{n+k-1}$, we say (3.17) is a linear $k$-step method. If $\beta_{k} \neq 0$, the method is called implicit and for $k=1$ we have a one-step method.

\section{Local truncation error}

Definition 9 (Local truncantion error [120]). The local truncation error is defined as the difference between the exact solution $\mathbf{x}\left(t_{n}+k\right)$ and its approximation $\mathbf{x}_{n+k}$

$$
\left|\mathbf{x}\left(t_{n}+k\right)-\mathbf{x}_{n+k}\right|,
$$

when applying the multistep method (3.17) to compute $\mathbf{x}_{n+k}$ with exact previous solutions $\mathbf{x}_{i}=\mathbf{x}\left(t_{i}\right)$, $i=0, \ldots, k-1$. The multistep method is called consistent, if the local truncation error tends to zero, whenever the step size $h$ tends to zero, that is

$$
\left|\mathbf{x}\left(t_{n}+k\right)-\mathbf{x}_{n+k}\right| \underset{h \rightarrow 0}{\longrightarrow} 0 .
$$

For a sketch of the local truncation error see Figure 3.4. The multistep method is of order $p$ if its LTE is $\mathcal{O}\left(h^{p+1}\right)$ [120]. As it can be observed in Figure 3.4, the LTE propagates through the time steps and amplifies the global error of the time integration scheme. Therefore, it is not sufficient to have information about the LTE to obtain a convergence statement about the time integration scheme in the whole simulation time window $\mathcal{I}$. 


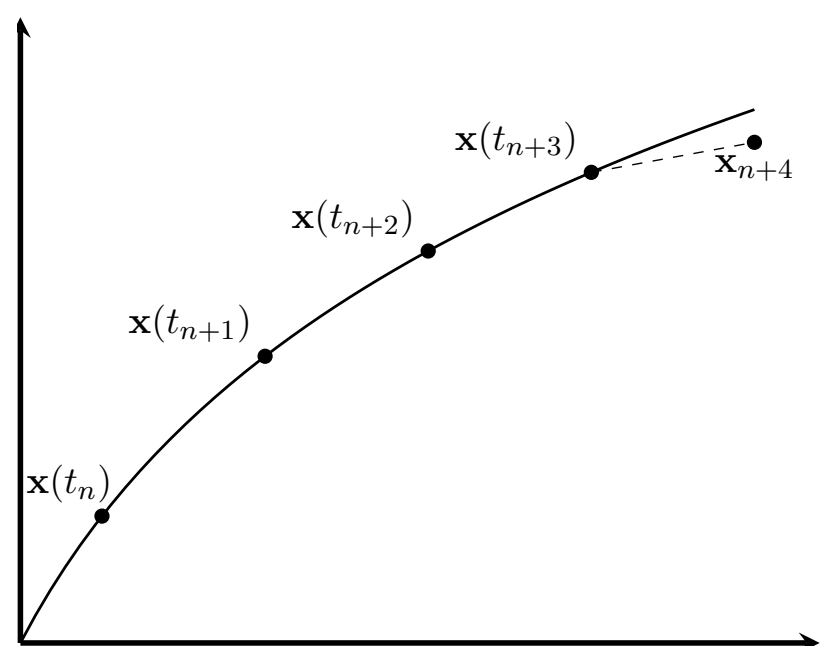

(a) Local truncation error.

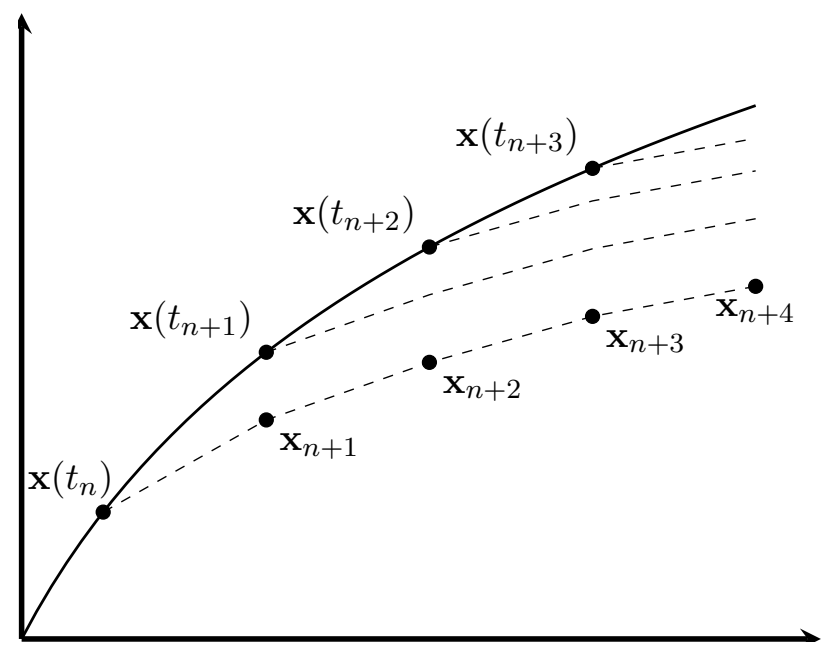

(b) Local truncation error propagation.

Figure 3.4: Sketch of local error propagation in the global error.

Definition 10 (Convergence of multistep method [120]). The linear $k$-step method (3.17) is convergent if for all IVP (3.16) with a Lipschitz continuous function $\mathbf{f}$,

$$
\mathbf{x}\left(t_{k}\right)-\mathbf{x}_{k} \underset{h \rightarrow 0}{\longrightarrow} 0
$$

whenever $\mathbf{x}\left(t_{i}\right)-\mathbf{x}_{i} \underset{h \rightarrow 0}{\longrightarrow} 0$, for all $i=0, \ldots, k-1$.

\section{Stability}

The concept of stability allows to, given a LTE, obtain statements about the convergence of the time integration scheme. Intuitively, it allows to judge whether the contribution of the LTE in the global error increases without control. It is proven that a stable and consistent method is convergent [120].

To exemplify how the stability analysis of a time integration method is performed, we will consider the case where $n_{\text {dof }}=1$ and present the study for the two Euler methods. For a more detailed explanation of how to perform such a study in more complicated cases (e.g. for multistep methods) see [120], [121].

Euler's method. Let us consider the initial value problem (3.16) for $t \in \mathcal{I}$ and its numerical integration with time steps $t_{i}, i=0, \ldots, n_{t}$. Then, for the

- explicit Euler scheme,

$$
\frac{1}{t_{i+1}-t_{i}}\left(\mathbf{x}_{i+1}-\mathbf{x}_{i}\right)=\mathbf{f}\left(\mathbf{x}_{i}, t_{i}\right)
$$

- implicit Euler scheme,

$$
\frac{1}{t_{i+1}-t_{i}}\left(\mathbf{x}_{i+1}-\mathbf{x}_{i}\right)=\mathbf{f}\left(\mathbf{x}_{i+1}, t_{i+1}\right)
$$


For the stability analysis of the time integration method, we apply it to Dahlquist's test equation [121]

$$
\frac{\mathrm{d}}{\mathrm{d} t} x=\lambda x, \quad x\left(t_{0}\right)=1,
$$

with $\lambda \in \mathbb{C}$, whose solution is given by the function $x(t)=e^{\lambda t}$.

Assuming a constant step size $t_{i+1}-t_{i}=h$, the aim is to obtain an expression of the type

$$
x_{i+1}=R(h \lambda) x_{i},
$$

when applying the time integration scheme to the test equation (3.20). This yields $x_{i+1}=R(h \lambda)^{i+1} x_{0}$, where $x_{i+1} \underset{i \rightarrow \infty}{\longrightarrow} 0$, if $|R(h \lambda)|<1$. For the numerical scheme, it is important to yield a stable solution that does not diverge for $i \rightarrow \infty$, whenever the exact solution of (3.20) is also stable.

Definition 11 (Stability domain [121]). The stability domain $S$ of the time integration scheme is defined as

$$
S=\{h \lambda \in \mathbb{C} ;|R(h \lambda)| \leq 1\} .
$$

Remark 12. For a more general definition of stability domain in the case of multistep methods see [121, Chapter 5].

Example 1. In the case of the two Euler methods,

$$
\begin{array}{ll}
R(h \lambda)=(1+h \lambda), & \text { for explicit Euler } \\
R(h \lambda)=\frac{1}{1-h \lambda}, & \text { for implicit Euler. }
\end{array}
$$

This yields stability regions of

$$
\begin{array}{ll}
S=\{h \lambda ;|1+h \lambda| \leq 1\} & \text { for explicit Euler } \\
S=\{h \lambda ;|1-h \lambda| \geq 1\} & \text { for implicit Euler. }
\end{array}
$$

A visualisation of the stability regions of both Euler methods can be seen in Figure 3.5.

Note that, whenever $\lambda$ has a negative real value, and therefore, the solution of the original ODE is stable, the explicit Euler requires the time step size $h$ to be $0 \leq h \leq \frac{-2}{\lambda}$ to be also stable. However, in the case of the implicit Euler, the scheme converges for all $h \geq 0$ and thus has no restriction on the time step size.

Definition 12. A linear $k$-step method (3.17) is A-stable if its stability region fulfils

$$
S \supseteq S_{\mathrm{A}}=\{h \lambda \in \mathbb{C} ; \Re(h \lambda)<0\} .
$$

For a sketch of the stability region $S_{\mathrm{A}}$ see Figure 3.6.

If a method is A-stable, the solution of the numerical method tends to zero, whenever the solution of the original ODE (3.20) also tends to zero, for all $h>0$ [123]. Therefore, the time step size is not determined by the stability condition of the numerical method, but rather by the required accuracy and the dynamics of the equation.

Definition 13 ([121]). A linear $k$-step method (3.17) is $\mathrm{A}(\alpha)$-stable if its stability region fulfils, for $0<\alpha<\frac{\pi}{2}$

$$
S \supseteq S_{\alpha}=\{h \lambda \in \mathbb{C} ;|\arg (-h \lambda)|<\alpha, h \lambda \neq 0\} .
$$

An example of an $\mathrm{A}(\alpha)$-stable region can be visualised in Figure 3.6. 

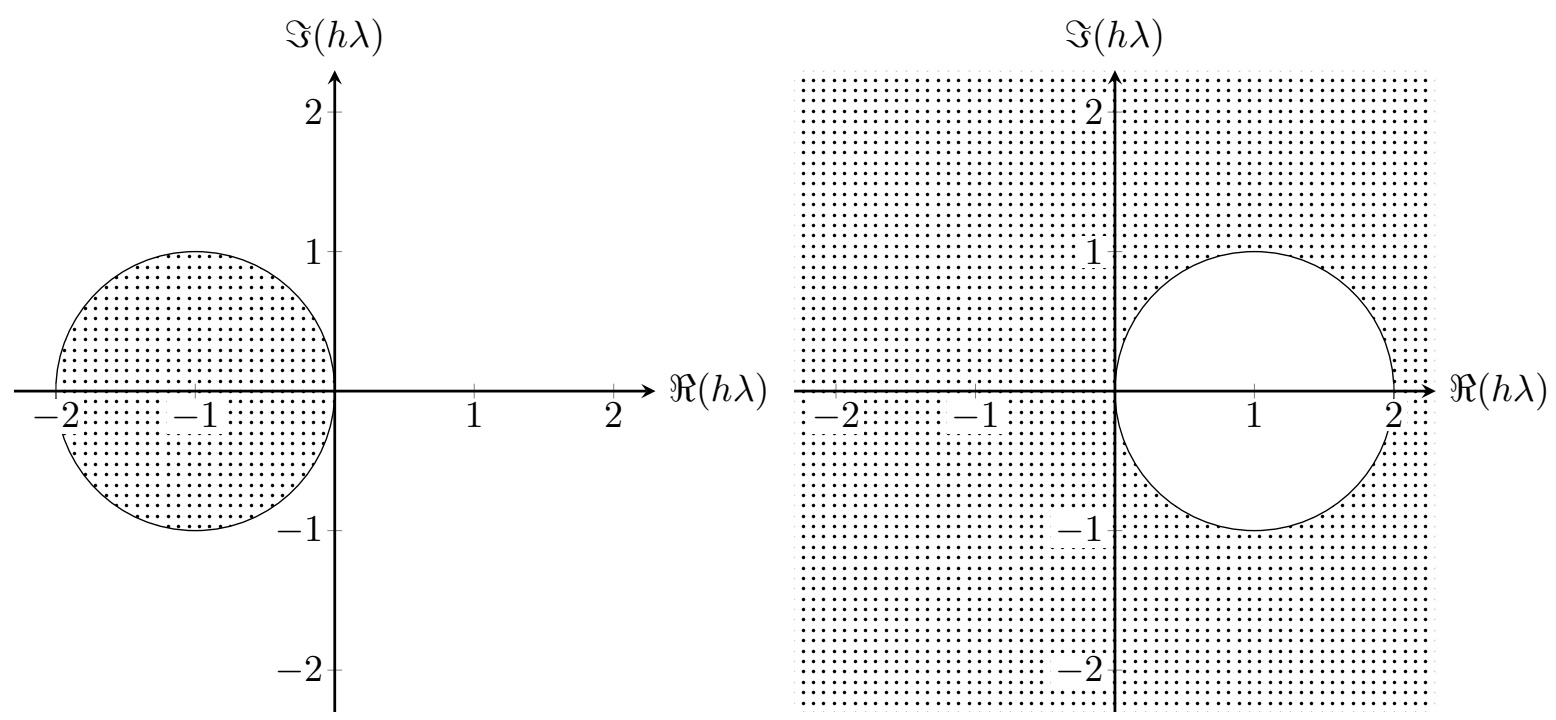

Figure 3.5: Stability region (dotted) of explicit Euler (left) and implicit Euler (right).

\section{Stiff equations}

Stiff equations are those that have special difficulties with the stability of the time integration methods. There is no formal definition of stiff equations, however, there are several ways of characterising them.

In [121], stiff equations are described as systems of differential equations, where explicit methods can not be used. Other works, such as [122] or [101] relate the stiffness to differential equations, where dynamics with different time-scales are present. There, the stability region of explicit methods constraints the time-step sizes, based on the faster dynamics. However, to also resolve the smaller dynamics, too many small timesteps would be required, which makes the resolution in practice infeasible. Let us consider a linear system of ODEs

$$
\frac{\mathrm{d}}{\mathrm{d} t} \mathbf{x}=\mathbf{A} \mathbf{x}+\mathbf{f}(t)
$$

where $\mathbf{x}: \mathcal{I} \rightarrow \mathbb{R}^{n}$ and $\mathbf{A} \in \mathbb{R}^{n \times n}$ is a diagonalizable matrix with eigenvalues $\lambda_{1}, \ldots, \lambda_{n} \in \mathbb{C}$. In [101], the two different time scales are characterised by the stiffness ratio, that is, $\left|\Re\left(\lambda_{\max }\right)\right| /\left|\Re\left(\lambda_{\min }\right)\right|$, where $\left|\Re\left(\lambda_{\max }\right)\right| \geq\left|\Re\left(\lambda_{i}\right)\right| \geq\left|\Re\left(\lambda_{\min }\right)\right|$, for all $i$. If this ratio is large enough, the system involves different timescales and thus is stiff. However, in [122], the behaviour is characterised by the interval length and the spectral radius of the matrix. Here, the linear system of ODEs is called stiff on $\mathcal{I}$, if $\left|\rho(\mathbf{A})\left(t_{\text {end }}-t_{0}\right)\right| \gg 1$, with spectral radius $\rho(\mathbf{A})=\max \left\{\lambda_{1}, \ldots, \lambda_{n}\right\}$.

For stiff equations, the stability region study is specially relevant to avoid the time step size to be determined by the stability of the method rather than the accuracy of the solution.

Differential algebraic equations (see Section 3.3) are sometimes perceived as infinitely stiff [124].

\subsubsection{Time integration techniques}

Most systems of differential equations presented in this work are stiff and therefore all time integration methods presented in the following are implicit ones. We consider, apart from the implicit Euler method 

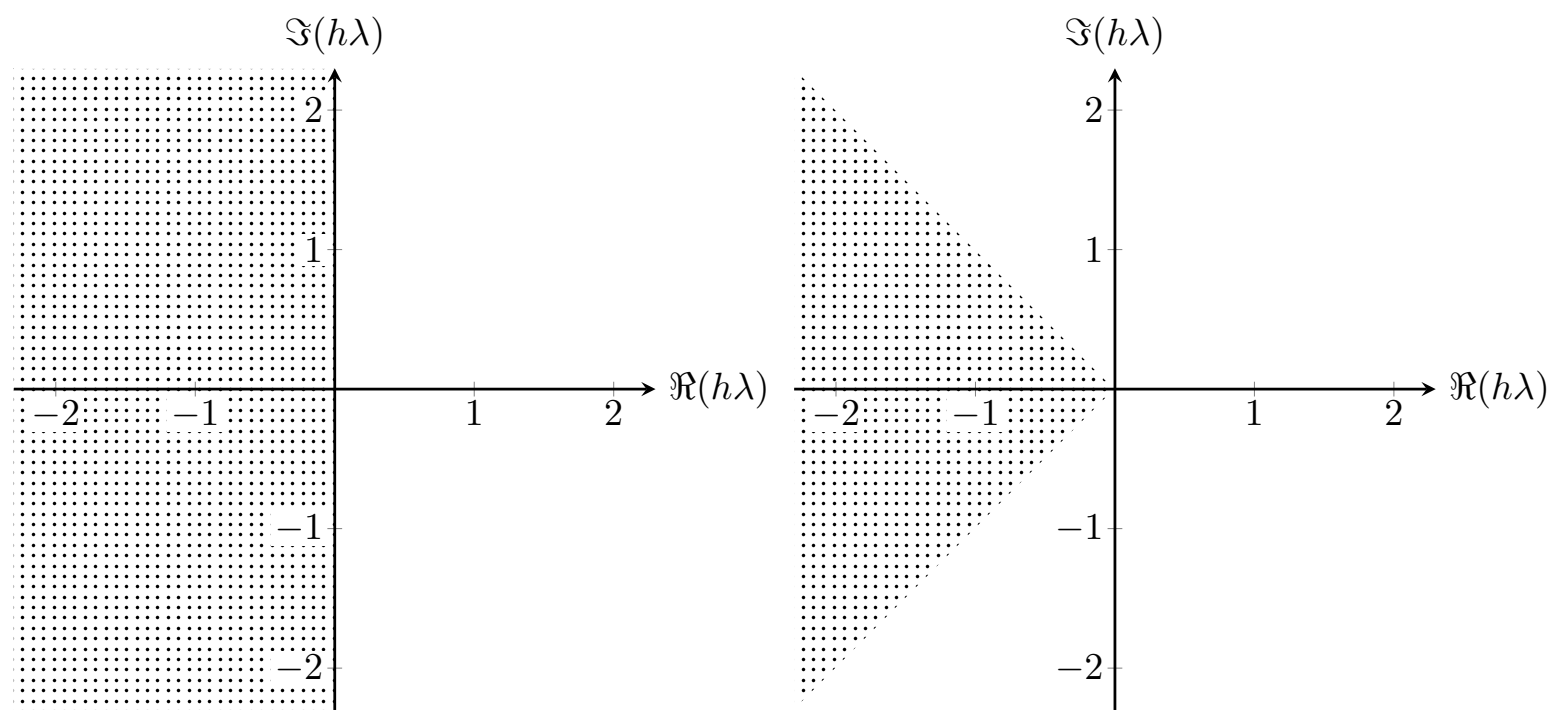

Figure 3.6: Stability regions (dotted) for an A-stable method (left) and an A( $\alpha)$-stable method, with $\alpha=\pi / 4$ (right).

(3.19), two main time integration techniques, the backward differentiation formula (BDF) and the trapezoidal rule. The following section describes methods itself, together with the known stability and convergence order results.

\section{Backward differentiation formula}

The backward differentiation formula is an implicit and linear multistep method. For an initial value problem of the form (3.16), the $k$-step BDF to approximate $\mathbf{x}_{i+1}$ reads with given $\mathbf{x}_{i+1-k}, \ldots, \mathbf{x}_{i}$, [125]

$$
\frac{1}{t_{i+1}-t_{i}} \sum_{j=0}^{k} \alpha_{i+1, j} \mathbf{x}_{i+1-j}-\mathbf{f}\left(\mathbf{x}_{i+1}, t_{i+1}\right)=0 \text {. }
$$

Here, $\alpha_{i+1, j}$ are determined by the polynomial $\mathbf{q}(t)$, which interpolates $\left(t_{j}, \mathbf{x}_{j}\right)$ for $j=i+1-k, \ldots, i+1$. To obtain (3.22), the time derivative at time point $t_{i+1}, \mathbf{x}_{i+1}^{\prime}$, is approximated by the derivative of the polynomial, such that

$$
\mathbf{q}^{\prime}\left(t_{i+1}\right)=\mathbf{f}\left(\mathbf{x}_{i+1}, t_{i+1}\right) .
$$

For a more detailed explanation of the method see [120].

Remark 13. Note that BDF-1 corresponds to the implicit Euler scheme (3.19).

The stability of BDF methods is well known. BDF-1 (order 1) and BDF-2 (order 2) are both A-stable. BDF with $k=3,4,5,6$ are only $\mathrm{A}(\alpha)$-stable, as stability on the imaginary axis is successively lost [121]. For $k \geq 7$ the method is unstable [120], [121]. For a summary of the properties of the different methods see Table 3.1. 


\begin{tabular}{|c||c|c|}
\hline Integration Method & Order & Stability \\
\hline Implicit Euler & 1 & A-stable \\
BDF-2 & 2 & A-stable \\
BDF-3 & 3 & A $(\alpha)$-stable \\
BDF-4 & 4 & A $(\alpha)$-stable \\
BDF-5 & 5 & A $(\alpha)$-stable \\
BDF-6 & 6 & A $(\alpha)$-stable \\
Trapezoidal Rule & 2 & A-stable \\
\hline
\end{tabular}

Table 3.1: Order and Stability of the numerical integration methods.

\section{Trapezoidal rule}

The trapezoidal rule for the IVP (3.16) can be derived by integrating the differential equation from $t_{i}$ to $t_{i+1}$ to obtain the following expression

$$
\mathbf{x}\left(t_{i+1}\right)=\mathbf{x}\left(t_{i}\right)+\int_{t_{i}}^{t_{i+1}} \mathbf{f}(\mathbf{x}, t) \mathrm{d} t
$$

Then, the integral on the right hand side of the previous expression is approximated by the trapezoidal rule, which yields the time integration scheme [120]

$$
\mathbf{x}_{i+1}=\mathbf{x}_{i}+\frac{t_{i+1}-t_{i}}{2}\left(\mathbf{f}\left(\mathbf{x}_{i}, t_{i}\right)+\mathbf{f}\left(\mathbf{x}_{i+1}, t_{i+1}\right)\right) .
$$

The method, which is obtained, is A-stable and has order 2 [120], [121] (see Table 3.1).

Remark 14. A theorem formulated by Dahlquist in 1963, states that A-stable multistep methods can not have order larger than 2 and that the smallest possible error constant $\left(c=\frac{1}{12}\right)$ is obtained with the trapezoidal rule [120], [123].

The trapezoidal rule can also be classified as an implicit Runga Kutta (IRK) method and, in particular, a Lobato IIIA IRK. For further details on implicit Runga Kutta methods see [120], [121].

\subsubsection{Solution of nonlinear systems}

Implicit time integration techniques such as the BDF formulas or the Trapezoidal rule yield, for nonlinear differential equations, nonlinear systems of equations that need to be resolved. To do so, root-finding algorithms such as the Newton-Raphson method can be employed. For further details on Newton's method for nonlinear equations see [126]. For the example of the trapezoidal rule (3.24), the (classic) Newton method yields in its $(k+1)$-th iteration

$$
\mathbf{x}_{i+1}^{k+1}=\mathbf{x}_{i+1}^{k}-\left(\mathbf{I}-\frac{t_{i+1}-t_{i}}{2} \mathbf{J}_{\mathbf{f}}\left(\mathbf{x}_{i+1}^{k}, t_{i+1}\right)\right)^{-1}\left(\mathbf{x}_{i+1}^{k}-\mathbf{x}_{i}-\frac{t_{i+1}-t_{i}}{2}\left(\mathbf{f}\left(\mathbf{x}_{i}, t_{i}\right)+\mathbf{f}\left(\mathbf{x}_{i+1}^{k}, t_{i+1}\right)\right)\right),
$$

with $\mathbf{J}_{\mathbf{f}}\left(\mathbf{x}_{i+1}^{k}, t_{i+1}\right)$ being the Jacobian of $\mathbf{f}(\mathbf{x}, t)$ in $\mathbf{x}$ evaluated at $\left(\mathbf{x}_{i+1}^{k}, t_{i+1}\right)$. The update that is obtained for each iteration contains the inverse of a term. The inverse, however, is not computed in practice as it is computationally prohibitively expensive. Instead, numerical techniques for the solution of systems of linear equations are used such as e.g. LU decomposition [127]. 


\subsection{Differential Algebraic Equations}

Even though the introduction to numerical integration techniques has only considered ODEs, most of the timedependent differential equations appearing in this work are differential algebraic equations.

DAEs are systems of equations where a set of ordinary differential equations is combined with algebraic constraints. In its most general form, they can be described with the following definition.

Definition 14 (Fully implicit DAE). We define a fully implicit system of differential algebraic equations as a system

$$
\mathbf{F}\left(\mathbf{x}^{\prime}, \mathbf{x}, t\right)=0
$$

on $t \in \mathcal{I}$, where $\operatorname{det}\left(\frac{\partial \mathbf{F}}{\partial \mathbf{x}^{\prime}}\right)=0$.

For a linear DAE

$$
\mathbf{A x} \mathbf{x}^{\prime}+\mathbf{B x}=\mathbf{f}(t)
$$

with a regular matrix pencil, that is, $\operatorname{det}(A+\lambda B) \neq 0$, for some $\lambda$, this corresponds to having a singular mass matrix A. Here, it can intuitively be seen, that this yields to a system containing some algebraic equations.

For linear DAEs (3.27) with regular matrix pencil it is known, that a solution of the DAE exists [124, Chapter 2]. Solvability for fully implicit DAEs (3.26) is not easy to determine [124], [125]. For a discussion on the existence of solution of nonlinear DAEs with special structures see [125, Chapter 3].

These types of systems of equations, involve numerical as well as analytical difficulties, that do not arise in classic ODE theory [128]. DAEs are, for example, specially sensitive towards perturbations [125], which complicates their numerical treatment. Also, the choice of appropriate initial conditions is not trivial, as they have to fulfil the algebraic constraints imposed by the DAE [125], [129].

\subsubsection{Index}

Systems of DAEs can be classified according to their index [125], [130]. The index is a natural number, which intuitively allows to deduce the numerical and analytical difficulties the DAE carries. Higher index DAEs have a more challenging treatment. There are different index concepts, that essentially coincide for regular, linear DAEs [130]. We present three different types, the projector-based tractability index [125], which is relevant for the study of appropriate initial conditions, the perturbation index [121], [124] and the differential index [124], [125].

Definition 15 (Differential index [124]). An implicit, solvable and sufficiently smooth system of DAEs (3.26) is said to have differential index $m$, if $m$ is the minimal number of analytical differentiations of (3.26),

$$
\frac{\mathrm{d}}{\mathrm{d} t} \mathbf{F}\left(\mathbf{x}^{\prime}, \mathbf{x}, t\right)=0, \ldots, \frac{\mathrm{d}^{m}}{\mathrm{~d} t^{m}} \mathbf{F}\left(\mathbf{x}^{\prime}, \mathbf{x}, t\right)=0,
$$

that are required to obtain, with only algebraic manipulations of (3.26) and (3.28), an ODE

$$
\mathbf{x}^{\prime}=\varphi(\mathbf{x}, t),
$$

with $\varphi$ being a continuous function in $\mathbf{x}$ and $t$. 
The definition can be thought of as a measure of how many time differentiations the DAE is away from an ODE. Thus, ODEs are index 0 DAEs [124, Chapter 2]. As numerical differentiation is an unstable process, and this time differentiation process is implicitly performed by the time integration scheme, instabilities and error magnification can occur.

Remark 15. During all the differential index analysis in this work, we will assume that the functions, that are involved, are sufficiently differentiable. Other index concepts, such as the tractability index, require less smoothness assumptions and thus could be used to relax this condition.

To illustrate the possible sensitivity towards small perturbations of DAEs with high index, we define the perturbation index concept.

Definition 16 (Perturbation index [121], [124]). The implicit, solvable and sufficiently smooth system of DAEs (3.26) has perturbation index $m$ along a solution $\mathbf{x}$ on $\mathcal{I}$, if $m$ is the smallest integer such that for a perturbed solution $\widehat{\mathbf{x}}$ with

$$
\mathbf{F}\left(\widehat{\mathbf{x}}^{\prime}, \widehat{\mathbf{x}}, t\right)=\delta(t)
$$

there exists the estimate on $\mathcal{I}$

$$
\|\mathbf{x}(t)-\widehat{\mathbf{x}}(t)\| \leq C\left(\left\|\mathbf{x}\left(t_{0}\right)-\widehat{\mathbf{x}}\left(t_{0}\right)\right\|+\max _{t_{0} \leq \xi \leq t}\|\delta(\xi)\|+\ldots+\max _{t_{0} \leq \xi \leq t}\left\|\delta^{(m-1)}(\xi)\right\|\right)
$$

for sufficiently small right-hand side $\delta(t)$.

Note that, for small perturbations with large time derivatives (e.g. with very high frequencies), the solution of the perturbed problem may differ a lot with respect to the original DAE, for systems with perturbation index $\geq 2$.

To exemplify this, let us consider the following index 2 DAE and its perturbed problem

$$
\begin{aligned}
x^{\prime}(t) & =y(t)-x(t) & \hat{x}^{\prime}(t) & =\hat{y}(t)-\hat{x}(t) \\
0 & =x(t)-\sin (t) & 0 & =\hat{x}(t)-\sin (t)-\varepsilon \sin (M t),
\end{aligned}
$$

with $0<\frac{1}{\varepsilon} \ll M$ and a small $\varepsilon$. Then, the solution of the original DAE is

$$
x(t)=\sin (t) \quad y(t)=\sin (t)+\cos (t),
$$

and the solution of the perturbed problem

$$
\hat{x}(t)=\sin (t)+\varepsilon \sin (M t) \quad \hat{y}(t)=\sin (t)+\cos (t)+\varepsilon \sin (M t)+M \varepsilon \cos (M t) .
$$

Here, a small, perturbation with high frequency yields a high variation in the solution of the perturbed system for $y(t)$ as

$$
|y(t)-\hat{y}(t)|=|\varepsilon \sin (M t)+M \varepsilon \cos (M t)|,
$$

with $M \varepsilon \gg 1$.

Remark 16. DAEs with index 0 or 1 do not pose high difficulties when handling them numerically and analytically. However, in DAEs with index $\geq 2$, also called higher index DAEs, more complications start arising and thus need special care. In the perturbation index concept this potentially problematic behaviour can be reflected by the fact that derivatives of the small perturbation $\delta(t)$ start affecting the error bound of the perturbed problem for index $m \geq 2$. 
Based on [129], for the next index concept we introduce a special type of DAE with more structure. All the systems considered during this work can be described by such DAEs. The generalisation of the tractability index to fully implicit nonlinear DAEs can be found in [125] and is based on a linearisation of the system.

We consider the quasilinear DAE

$$
\mathbf{A}(\mathbf{x}, t) \mathbf{x}^{\prime}+\mathbf{b}(\mathbf{x}, t)=0
$$

with projectors

$$
\mathbf{Q}(t) \text { onto ker } \mathbf{A}(\mathbf{x}, t) \quad \text { and } \quad \mathbf{P}(t)=\mathbf{I}-\mathbf{Q}(t) .
$$

Assumption 12 (Kernel and Image of mass matrix [129]). We assume that both the spaces $\mathrm{ker} \mathbf{A}(\mathbf{x}, t)$ and $\operatorname{im} \mathbf{A}(\mathbf{x}, t)$ as well as the projectors $\mathbf{Q}(t)$ and $\mathbf{P}(t)$ depend smoothly on $t$ and are independent of $\mathbf{x}$.

In addition, we define the matrices

$$
\begin{aligned}
\mathbf{B}(\mathbf{y}, \mathbf{x}, t) & :=\frac{\partial}{\partial \mathbf{x}}(\mathbf{A}(\mathbf{x}, t) \mathbf{y})+\frac{\partial}{\partial \mathbf{x}} \mathbf{b}(\mathbf{x}, t) \\
\mathbf{A}_{1}(\mathbf{y}, \mathbf{x}, t) & :=(\mathbf{A}(\mathbf{x}, t)+\mathbf{B}(\mathbf{y}, \mathbf{x}, t) \mathbf{Q}(t))\left(\mathbf{I}-\mathbf{P}(t) \mathbf{P}^{\prime}(t) \mathbf{Q}(t)\right)
\end{aligned}
$$

and the projectors

$$
\mathbf{Q}_{1}(\mathbf{y}, \mathbf{x}, t) \text { onto ker } \mathbf{A}_{1}(\mathbf{y}, \mathbf{x}, t) \quad \text { and } \quad \mathbf{P}_{1}(t)(\mathbf{y}, \mathbf{x}, t)=\mathbf{I}-\mathbf{Q}_{1}(\mathbf{y}, \mathbf{x}, t) .
$$

Definition 17 (Tractability index [125], [129]). The quasilinear DAE (3.29) with projectors fulfilling Assumption 12 has tractability

(i) index 0 , if $\mathbf{A}(\mathbf{x}, t)$ is nonsingular.

(ii) index 1, if $\mathbf{A}(\mathbf{x}, t)$ is singular and $\mathbf{A}_{1}(\mathbf{y}, \mathbf{x}, t)$ is nonsingular.

(iii) index 2 , if $\mathbf{A}_{1}(\mathbf{y}, \mathbf{x}, t)$ is singular, and

$$
\mathbf{G}_{2}(\mathbf{y}, \mathbf{x}, t):=\mathbf{A}_{1}(\mathbf{y}, \mathbf{x}, t)+\mathbf{B}(\mathbf{y}, \mathbf{x}, t) \mathbf{P}(t) \mathbf{Q}_{1}(\mathbf{y}, \mathbf{x}, t)
$$

is nonsingular.

Only spaces and projectors for at most index 2 tractable DAEs are relevant for this work. Therefore, we do not expand the definition of tractability index for DAEs with index 3 or higher. For a generalisation of the sequence of matrices to define $m$ tractable index DAEs see [125].

\subsubsection{Initial conditions}

When considering initial value problems with ODEs, arbitrary ICs lead to a valid solution of the system. However, for the case of DAEs, the choice of initial conditions is not trivial, as not every IC is part of a solution of the system. Whenever talking about appropriate initial conditions the term consistent is used.

Definition 18 (Consistent initial condition). An initial condition $\mathbf{x}_{0} \in \mathbb{R}^{n_{\mathrm{dof}}}$ at $t_{0}$ for the DAE (3.26) is called consistent, if there exists a solution $\overline{\mathbf{x}}: \mathcal{I} \rightarrow \mathbb{R}^{n_{\mathrm{dof}}}$ of the system (3.26), such that $\overline{\mathbf{x}}\left(t_{0}\right)=\mathbf{x}_{0}$. 
To exemplify the difficulties that can arise regarding consistent initialisation, let us consider the system of DAEs

$$
\begin{aligned}
x^{\prime}(t) & =y(t) \\
0 & =x(t)-f(t),
\end{aligned}
$$

where $x$ and $z$ are the degrees of freedom, and $f(t)$ is a given function. Clearly, the second equation poses an explicit constraint to $x(t)$, which the initial condition has to fulfil, therefore

$$
x_{0}=f\left(t_{0}\right)
$$

is fixed by the system. Differentiating the second equation once and plugging it into the first one yields the hidden constraint $y\left(t_{0}\right)=f^{\prime}\left(t_{0}\right)$, which also has to be fulfilled by the initial condition. Therefore, in this case

$$
y_{0}=f^{\prime}\left(t_{0}\right)
$$

and all initial values are fixed.

Index 1 DAEs only have explicit constraints and are therefore easier to initialise, as only the explicit algebraic equations that are defined by the DAE have to be fulfilled. However, higher index DAEs have these type of hidden algebraic constraints, that both complicate the initialisation as well as involve possible differentiations of inputs. Consistent initial conditions have to fulfil all the explicit as well as implicit algebraic constrains imposed by the DAE.

\subsubsection{Numerical methods for DAEs}

The application of the classic time discretisation schemes for ODEs to systems of the form (3.29), might not be straightforward. A priori, they are defined for systems with an explicit expression for the time derivative of the degrees of freedom, that is, for $\mathbf{A}(\mathbf{x}, t)=\mathbf{I}$. Furthermore, the convergence results of the different methods are not immediately applicable to arbitrary DAEs. In the following part, we will deal with the generalisation of the time integration results presented in Section 3.2 when used with DAEs.

Let us again consider the quasilinear DAE initial value problem with constant rank matrix $\mathbf{A}(\mathbf{x}, t)$

$$
\begin{aligned}
\mathbf{A}(\mathbf{x}, t) \frac{\mathrm{d}}{\mathrm{d} t} \mathbf{x}+\mathbf{b}(\mathbf{x}, t) & =0, \\
\mathbf{x}\left(t_{0}\right) & =\mathbf{x}_{0},
\end{aligned}
$$

for $t \in \mathcal{I}$ and consistent initial condition $\mathbf{x}_{0}$ at $t_{0}$.

\section{Backward differentiation formula}

The generalisation of the BDF- $k$ formula explained in Section 3.2.2 to the quasilinear DAE IVP (3.31) follows straightforward, by replacing the time derivative of $\mathbf{x}, \mathbf{x}^{\prime}$ in (3.31a) with the derivative of the interpolation polynomial, for time step $t_{i}+1$, i.e.

$$
\begin{aligned}
0 & =\mathbf{A}\left(\mathbf{x}_{i+1}, t_{i+1}\right) \mathbf{q}^{\prime}\left(t_{i+1}\right)+\mathbf{b}\left(\mathbf{x}_{i+1}, t_{i+1}\right) \\
& =\mathbf{A}\left(\mathbf{x}_{i+1}, t_{i+1}\right) \frac{1}{t_{i+1}-t_{i}} \sum_{j=0}^{k} \alpha_{i+1, j} \mathbf{x}_{i+1-j}+\mathbf{b}\left(\mathbf{x}_{i+1}, t_{i+1}\right) .
\end{aligned}
$$


The stability and convergence of such methods on arbitrary DAEs is not ensured. Most of the convergence results are given for constant step size BDF methods, as changes in the stepsize, specially in the implicit Euler case, can yield to accuracy problems [124, Chapter 3]. Even for linear index 3 DAEs with constant coefficients, implicit Euler with variable step sizes can contain $\mathcal{O}(1)$ errors [124, Chapter 3].

In [124, Chapter 3], convergence order results for fixed step size BDF- $k$ methods applied to special structured DAEs are shown. BDF- $k$ methods, for $k<7$ with fixed step size have convergence order $\mathcal{O}\left(h^{k}\right)$ for IVPs with the structure (3.31), provided the initial conditions and the root finding problem $\mathbf{F}\left(\mathbf{x}_{i+1}\right)=0$ from (3.32) are $\mathcal{O}\left(h^{k+1}\right)$ accurate. In this cases it is also shown, that variable step size BDF can also be convergent and stable, provided some conditions on the step size control are fulfilled (c.f. [124]).

Remark 17. For a specific type of index 3 systems (in Hessenberg form), similar convergence order results can be shown, provided enough accuracy is ensured in the solution of the algebraic equations (see [124, Chapter 3]).

\section{Trapezoidal rule}

The generalisation of the Trapezoidal rule to DAEs does not follow as directly as in the case of the BDF. We apply the Trapezoidal rule at time $t_{i+1}$ for the quasilinear DAE IVP (3.31) with constant mass matrix $\mathbf{A}$ and the $\varepsilon$-embedding method (see [121]) as

$$
0=\mathbf{A}\left(\mathbf{x}_{i+1}-\mathbf{x}_{i}\right)+\frac{h}{2}\left(\mathbf{b}\left(\mathbf{x}_{i}, t_{i}\right)+\mathbf{b}\left(\mathbf{x}_{i+1}, t_{i+1}\right)\right) .
$$

The theory for the convergence of this method for DAEs is less detailed than for BDF schemes. Inside the IRK methods, it can be classified as a Lobatto IIIA method and for these specific type of methods, in [121], [131] it is shown, that applied to index 1 quasilinear DAEs with constant mass matrix as shown in (3.33), the method converges with order $\mathcal{O}\left(h^{2}\right)$, provided consistent initial conditions are given. For the case of index 2 systems, convergence proofs are available for DAEs with specific structure, such as systems in Hessenberg form (see [121]). 


\section{Structural Analysis of the Coupled Systems}

As it has been mentioned in Section 3.3, higher index DAEs require special numerical treatment. Therefore, when dealing with (coupled) systems of DAEs, a priori knowledge about their index allows to properly handle their simulation. In particular when using more fragile algorithms such as co-simulation techniques or parallel-in-time methods, the appropriate treatment of the systems is of utmost importance to ensure the (correct) convergence of the methods.

Electric networks described with modified nodal analysis yield a system of DAEs. Its tractability and differential index for circuits containing classic elements, i.e. resistances, inductances, capacitances and sources, is well known and depends only on topological properties of the circuit [18]. However, very complex models can often not be described by the classic elements and require more involved equations to properly characterise them. In these cases refined modelling can be performed, where the system of equations describing the circuit is coupled to a PDE describing the spatially resolved electromagnetic behaviour of the complex element [2], [11], [15], [16], [31]. An example for such an application is the simulation of the LHC's quench protection system [5] where, not only an eddy current PDE is coupled to MNA, but also other effects such as heat propagation are described through refined models.

Whenever the index study of circuits with refined models is carried out, similar analysis as in [18] needs to be expanded for each different model individually. In [19], [21], [22], the index of semidiscrete parabolic eddy current formulations coupled to circuits is studied, [23] deals with the analysis of semiconductor models in circuits and [20] performs an index study of full Maxwell's equations with different gauging conditions.

To avoid the repetition of the analysis of the coupled system's DAE for each refined model such that it can be coupled to electric networks, three generalised element definitions are presented. They allow to, similarly as in [18], classify the different refined elements and then deduce index results of the coupled system through only topological properties of the circuit. In [55], the definition of an inductance-like element is presented together with two different eddy current formulations that can be classified as such. In [24], an alternative definition of inductance-like element is given, extending the previous definition, which allows to also include an $\vec{A}-\phi$ formulation of full Maxwell's equations. In addition, definitions for a capacitance-like element and a resistance-like element are introduced together with two different refined models that can be classified as such.

This chapter follows [24], [55] and presents three generalised elements definitions as well as the index analysis of the DAE system of equations arising from MNA containing the generalised elements. For each one of the definitions, examples arising from different approximations of Maxwell's equations are given. The chapter is structured as follows: Following the first definition of inductance-like element from [55], Section 4.1 defines the three different generalised elements. Here, it is also shown that all classical circuit elements from the MNA index analysis in [18], as well as the flux-charge formulated ones can be classified as their corresponding generalisation. Section 4.2 presents the DAE that arises from a circuit described with modified nodal analysis and containing the generalised circuit elements. It introduces the necessary assumptions to formulate the corresponding index analysis results. Finally, a special structure for inductance-like elements is given 
that ensures, analogous to classic MNA, the linearity of the index 2 components. Section 4.3 introduces the semidiscrete systems of equations arising from the different approximations and formulations of Maxwell's equations and classifies them according to the generalised elements' definitions. Section 4.4 concludes the chapter with a summary.

\subsection{Generalised Circuit Elements}

Even though [24] presents more general results, we follow [55] to define our inductance-like element and, based on that structure, also give definitions for capacitance-like and resistance-like elements. This yields a more intuitive structure for the generalised elements, that requires less a priori mathematical fundamentals. For mathematically more involved definitions that allow to also classify full Maxwell's equations described with the $\vec{A}-\phi$ formulation we refer to [24]. The structure and content of this section follows [24], [55].

\subsubsection{Definitions}

Definition 19 (Inductance-like element [55]). We define an inductance-like element with $n_{\mu}$ ports as one described by a system of DAEs

$$
\mathbf{F}_{\mu}\left(\frac{\mathrm{d}}{\mathrm{d} t} \mathbf{d}_{\mu}\left(\mathbf{x}_{\mu}, \mathbf{i}_{\mu}\right), \mathbf{x}_{\mu}, \mathbf{i}_{\mu}, \mathbf{v}_{\mu}, t\right)=0
$$

with $\mathbf{x}_{\mu}: \mathcal{I} \rightarrow \mathbb{R}^{n_{\text {dof }}}$ and $\mathbf{i}_{\mu}, \mathbf{v}_{\mu}: \mathcal{I} \rightarrow \mathbb{R}^{n_{\mu}}$, such that there is at most one time differentiation

$$
\frac{\mathrm{d}}{\mathrm{d} t} \mathbf{F}_{\mu}\left(\frac{\mathrm{d}}{\mathrm{d} t} \mathbf{d}_{\mu}\left(\mathbf{x}_{\mu}, \mathbf{i}_{\mu}\right), \mathbf{x}_{\mu}, \mathbf{i}_{\mu}, \mathbf{v}_{\mu}, t\right)=0
$$

needed, to write only with algebraic manipulations of (4.1)-(4.2) a system

$$
\begin{aligned}
\frac{\mathrm{d}}{\mathrm{d} t} \mathbf{x}_{\mu} & =\mathbf{f}_{\mathbf{x}}\left(\mathbf{x}_{\mu}, \mathbf{i}_{\mu}, \mathbf{v}_{\mu}, t\right) \\
\frac{\mathrm{d}}{\mathrm{d} t} \phi_{\mu}\left(\mathbf{x}_{\mu}, \mathbf{i}_{\mu}, t\right) & =\mathbf{f}_{\phi}\left(\mathbf{x}_{\mu}, \mathbf{i}_{\mu}, \mathbf{v}_{\mu}, t\right),
\end{aligned}
$$

fulfilling the properties

(i) $\frac{\partial}{\partial \mathbf{i}_{\mu}} \phi_{\mu}\left(\mathbf{x}_{\mu}, \mathbf{i}_{\mu}, t\right)$ is nonsingular.

(ii) $\mathbf{L}_{\mu}\left(\mathbf{x}_{\mu}, \mathbf{i}_{\mu}, \mathbf{x}_{\mu}, t\right):=\frac{\partial}{\partial \mathbf{v}_{\mu}}\left(\left(\frac{\partial \phi}{\partial \mathbf{i}_{\mu}}\right)^{-1}\left(-\frac{\partial \phi}{\partial \mathbf{x}_{\mu}} \mathbf{f}_{\mathbf{x}}-\frac{\partial \phi}{\partial t}+\mathbf{f}_{\phi}\right)\right)$ is positive definite.

Note that in this definition, the DAE (4.1) does not depend on the time derivative of the voltage $\mathbf{v}_{\mu}$.

Example 2. For the first basic examples we verify that the two essential formulations for lumped inductances fulfilling Assumption 9 are inductance-like elements. 
1. Classical inductances, described with the equation (2.47)

$$
\mathbf{v}_{\mathrm{L}}=\frac{\mathrm{d}}{\mathrm{d} t} \phi_{\mathrm{L}}\left(\mathbf{i}_{\mathrm{L}}, t\right)
$$

are inductance-like elements. Note that this differential equation has the structure of (4.1) and, without the need of any time differentation, is already written as a system (4.3)-(4.4), where $\mathbf{x}_{\mu}=\{\}, \mathbf{f}_{\mathbf{x}}=\{\}$, $\mathbf{i}_{\mu}=\mathbf{i}_{\mathrm{L}}$ and $\mathbf{v}_{\mu}=\mathbf{v}_{\mathrm{L}}$. Due to Assumption $9, \frac{\partial}{\partial \mathbf{i}_{\mathrm{L}}} \phi_{\mathrm{L}}\left(\mathbf{i}_{\mathrm{L}}, t\right)=\mathbf{L}\left(\mathbf{i}_{\mathrm{L}}, t\right)$ is positive definite and thus fulfils both properties (i) and (ii) of Definition 19.

2. Flux formulated inductances

$$
\begin{aligned}
\mathbf{v}_{\mathrm{L}} & =\frac{\mathrm{d}}{\mathrm{d} t} \phi \\
\phi & =\phi_{\mathrm{L}}\left(\mathbf{i}_{\mathrm{L}}, t\right),
\end{aligned}
$$

are inductance-like elements. This time, the original DAE has to be differentiated once to obtain a system like (4.3)-(4.4)

$$
\frac{\mathrm{d}}{\mathrm{d} t} \phi=\mathbf{v}_{\mathrm{L}} \quad \frac{\mathrm{d}}{\mathrm{d} t} \phi_{\mathrm{L}}\left(\mathbf{i}_{\mathrm{L}}, t\right)=\mathbf{v}_{\mathrm{L}},
$$

where $\mathbf{x}_{\mu}=\phi$ and $\mathbf{f}_{\mathbf{x}}=\mathbf{v}_{\mathrm{L}}$. The properties (i) and (ii) of Definition 19 are obtained analogous to the classical inductance.

Definition 20 (Capacitance-like element). We define a capacitance-like element with $n_{\varepsilon}$ ports as one described by a system of DAEs

$$
\mathbf{F}_{\varepsilon}\left(\frac{\mathrm{d}}{\mathrm{d} t} \mathbf{d}_{\varepsilon}\left(\mathbf{x}_{\varepsilon}, \mathbf{v}_{\varepsilon}\right), \mathbf{x}_{\varepsilon}, \mathbf{i}_{\varepsilon}, \mathbf{v}_{\varepsilon}, t\right)=0,
$$

with $\mathbf{x}_{\varepsilon}: \mathcal{I} \rightarrow \mathbb{R}^{n_{\text {dof }}}$ and $\mathbf{i}_{\varepsilon}, \mathbf{v}_{\varepsilon}: \mathcal{I} \rightarrow \mathbb{R}^{n_{\varepsilon}}$, such that there is at most one time differentiation

$$
\frac{\mathrm{d}}{\mathrm{d} t} \mathbf{F}_{\varepsilon}\left(\frac{\mathrm{d}}{\mathrm{d} t} \mathbf{d}_{\varepsilon}\left(\mathbf{x}_{\varepsilon}, \mathbf{v}_{\varepsilon}\right), \mathbf{x}_{\varepsilon}, \mathbf{i}_{\varepsilon}, \mathbf{v}_{\varepsilon}, t\right)=0
$$

needed, to write only with algebraic manipulations of (4.5)-(4.6) a system

$$
\begin{aligned}
\frac{\mathrm{d}}{\mathrm{d} t} \mathbf{x}_{\varepsilon} & =\mathbf{g}_{\mathbf{x}}\left(\mathbf{x}_{\varepsilon}, \mathbf{i}_{\varepsilon}, \mathbf{v}_{\varepsilon}, t\right) \\
\frac{\mathrm{d}}{\mathrm{d} t} \mathbf{q}_{\varepsilon}\left(\mathbf{x}_{\varepsilon}, \mathbf{v}_{\varepsilon}, t\right) & =\mathbf{g}_{\mathbf{q}}\left(\mathbf{x}_{\varepsilon}, \mathbf{i}_{\varepsilon}, \mathbf{v}_{\varepsilon}, t\right),
\end{aligned}
$$

fulfilling the properties

(i) $\frac{\partial}{\partial \mathbf{v}_{\varepsilon}} \mathbf{q}_{\varepsilon}\left(\mathbf{x}_{\varepsilon}, \mathbf{v}_{\varepsilon}, t\right)$ is nonsingular.

(ii) $\mathbf{C}_{\varepsilon}\left(\mathbf{x}_{\varepsilon}, \mathbf{v}_{\varepsilon}, t\right)$ is positive definite, where $\frac{\mathrm{d}}{\mathrm{d} t} \mathbf{v}_{\varepsilon}=\mathbf{g}_{\mathbf{v}}\left(\mathbf{x}_{\varepsilon}, \mathbf{v}_{\varepsilon}, t\right)+\mathbf{C}_{\varepsilon}^{-1}\left(\mathbf{x}_{\varepsilon}, \mathbf{v}_{\varepsilon}, t\right) \mathbf{i}_{\varepsilon}$ is obtained by inserting (4.7) into (4.8).

Notice that

$$
\begin{aligned}
\frac{\mathrm{d}}{\mathrm{d} t} \mathbf{v}_{\varepsilon} & =\left(\frac{\partial}{\partial \mathbf{v}_{\varepsilon}} \mathbf{q}_{\varepsilon}\left(\mathbf{x}_{\varepsilon}, \mathbf{v}_{\varepsilon}, t\right)\right)^{-1}\left(-\frac{\partial}{\partial \mathbf{x}_{\varepsilon}} \mathbf{q}_{\varepsilon}(\ldots) \mathbf{g}_{\mathbf{x}}(\ldots)-\frac{\partial}{\partial t} \mathbf{q}(\ldots)+\mathbf{g}_{\mathbf{q}}(\ldots)\right) \\
& :=\mathbf{g}_{\mathbf{v}}\left(\mathbf{x}_{\varepsilon}, \mathbf{v}_{\varepsilon}, t\right)+\mathbf{C}_{\varepsilon}^{-1}\left(\mathbf{x}_{\varepsilon}, \mathbf{v}_{\varepsilon}, t\right) \mathbf{i}_{\varepsilon} .
\end{aligned}
$$


For these elements, the DAEs (4.5) does not depend on the time derivative of the current $\mathbf{i}_{\varepsilon}$.

Example 3. Analogously to the inductance examples, we demonstrate that the two common lumped formulations for capacitances fulfilling Assumption 9 are capacitance-like elements.

1. Classical capacitances, described by the equation (2.48)

$$
\mathbf{i}_{\mathrm{C}}=\frac{\mathrm{d}}{\mathrm{d} t} \mathbf{q}_{\mathrm{C}}\left(\mathbf{v}_{\mathrm{C}}, t\right)
$$

are capacitance-like elements. Again, the differential equation has the structure of (4.5) and, without the need of any time differentation, we obtain a system (4.7)-(4.8) with $\mathbf{x}_{\varepsilon}=\{\}, \mathbf{g}_{\mathbf{x}}=\{\}, \mathbf{i}_{\varepsilon}=\mathbf{i}_{\mathrm{C}}$ and $\mathbf{v}_{\varepsilon}=\mathbf{v}_{\mathrm{C}}$. Properties (i) and (ii) of Definition 19 are fulfilled as $\mathbf{C}_{\varepsilon}\left(\mathbf{x}_{\varepsilon}, \mathbf{v}_{\varepsilon}, t\right)=\frac{\partial}{\partial \mathbf{v}_{\mathrm{C}}} \mathbf{q}_{\mathrm{C}}\left(\mathbf{v}_{\mathrm{C}}, t\right)=\mathbf{C}\left(\mathbf{i}_{\mathrm{C}}, t\right)$ is positive definite according to Assumption 9.

2. Similarly, charge formulated capacitances

$$
\begin{aligned}
\mathbf{i}_{\mathrm{C}} & =\frac{\mathrm{d}}{\mathrm{d} t} \mathbf{q} \\
\mathbf{q} & =\mathbf{q}_{\mathrm{C}}\left(\mathbf{v}_{\mathrm{C}}, t\right),
\end{aligned}
$$

are capacitance-like elements with $\mathbf{x}_{\varepsilon}=\mathbf{q}$. The system (4.7)-(4.8) is again obtained after one time differentiation of the original DAE and $\mathbf{g}_{\mathrm{x}}=\mathbf{i}_{\mathrm{C}}$. Properties (i) and (ii) follow analogously from Assumption 9.

Definition 21 (Resistance-like element). We define a resistance-like element with $n_{\rho}$ ports as one described by a system of DAEs

$$
\mathbf{F}_{\rho}\left(\frac{\mathrm{d}}{\mathrm{d} t} \mathbf{d}_{\rho}\left(\mathbf{x}_{\rho}, \mathbf{i}_{\rho}, \mathbf{v}_{\rho}\right), \mathbf{x}_{\rho}, \mathbf{i}_{\rho}, \mathbf{v}_{\rho}, t\right)=0,
$$

with $\mathbf{x}_{\rho}: \mathcal{I} \rightarrow \mathbb{R}^{n_{\text {dof }}}$ and $\mathbf{i}_{\rho}, \mathbf{v}_{\rho}: \mathcal{I} \rightarrow \mathbb{R}^{n_{\rho}}$, such that there is at most one time differentiation

$$
\frac{\mathrm{d}}{\mathrm{d} t} \mathbf{F}_{\rho}\left(\frac{\mathrm{d}}{\mathrm{d} t} \mathbf{d}_{\rho}\left(\mathbf{x}_{\rho}, \mathbf{i}_{\rho}, \mathbf{v}_{\rho}\right), \mathbf{x}_{\rho}, \mathbf{i}_{\rho}, \mathbf{v}_{\rho}, t\right)=0
$$

needed to write only with algebraic manipulations of (4.9)-(4.10) a system

$$
\begin{aligned}
\frac{\mathrm{d}}{\mathrm{d} t} \mathbf{x}_{\rho} & =\mathbf{h}_{\mathbf{x}}\left(\mathbf{x}_{\rho}, \mathbf{i}_{\rho}, \mathbf{v}_{\rho}, t\right) \\
\frac{\mathrm{d}}{\mathrm{d} t} \mathbf{r}_{\rho}\left(\mathbf{x}_{\rho}, \mathbf{i}_{\rho}, \mathbf{v}_{\rho}, t\right) & =\mathbf{h}_{\mathbf{r}}\left(\mathbf{x}_{\rho}, \mathbf{i}_{\rho}, \mathbf{v}_{\rho}, t\right)
\end{aligned}
$$

fulfilling the properties

(i) $\frac{\partial}{\partial \mathbf{i}_{\rho}} \mathbf{r}\left(\mathbf{x}_{\rho}, \mathbf{i}_{\rho}, \mathbf{v}_{\rho}, t\right)$ is nonsingular.

(ii) $\mathbf{G}_{\rho}\left(\mathbf{x}_{\rho}, \mathbf{i}_{\rho}, \mathbf{v}_{\rho}, t\right):=-\left(\frac{\partial}{\partial \mathbf{i}_{\rho}} \mathbf{r}_{\rho}\left(\mathbf{x}_{\rho}, \mathbf{i}_{\rho}, \mathbf{v}_{\rho}, t\right)\right)^{-1} \frac{\partial}{\partial \mathbf{v}_{\rho}} \mathbf{r}_{\rho}\left(\mathbf{x}_{\rho}, \mathbf{i}_{\rho}, \mathbf{v}_{\rho}, t\right)$ is positive definite.

This time the DAE (4.9) can depend both on the time derivative of the voltage $\mathbf{v}_{\rho}$, as well as the time derivative of the current $\mathbf{i}_{\rho}$. 
Example 4. Let us verify that classic resistances (2.49) fulfilling Assumption 9 and written as

$$
\mathbf{i}_{\mathrm{R}}=\mathbf{g}_{\mathrm{R}}\left(\mathbf{v}_{\mathrm{R}}, t\right)
$$

are resistance-like elements. One time differentiation is required to obtain a system (4.11)-(4.12)

$$
\frac{\mathrm{d}}{\mathrm{d} t} \mathbf{i}_{\mathrm{R}}=\frac{\partial}{\partial \mathbf{v}_{\mathrm{R}}} \mathbf{g}_{\mathrm{R}}\left(\mathbf{v}_{\mathrm{R}}, t\right) \frac{\mathrm{d}}{\mathrm{d} t} \mathbf{v}_{\mathrm{R}}
$$

which again fulfils properties (i) and (ii) of Definition 21 due to Assumption 9.

Assumption 13 (Uniquely solvable DAEs). We assume all the (generalised) circuit elements described with DAEs like

$$
\mathbf{F}_{\star}\left(\frac{\mathrm{d}}{\mathrm{d} t} \mathbf{d}_{\star}\left(\mathbf{x}_{\star}, \mathbf{i}_{\star}, \mathbf{v}_{\star}\right), \mathbf{x}_{\star}, \mathbf{i}_{\star}, \mathbf{v}_{\star}, t\right)=0,
$$

are well posed in the sense that, for either a given current excitation

$$
\mathbf{i}_{\star}=\mathbf{f}_{\star}(t)
$$

or voltage excitation

$$
\mathbf{v}_{\star}=\mathbf{g}_{\star}(t),
$$

the initial value problem formed by (4.13) with (4.14) or (4.15) and some consistent initial conditions for $\mathbf{x}_{\star}$, $\mathbf{i}_{\star}$ and $\mathbf{v}_{\star}$ is uniquely solvable.

A multiport element could, in a general case, be structurally different on each port and thus behave, for example, like a capacitance-like element on one port and an inductance-like element on the other. In addition, it could also change its structure depending on the state variables, time or frequency. These cases, however, are not considered in the generalised element definitions presented here and could yield to an index that depends not only on the topology of the circuit, but also on other properties like e.g. time.

Remark 18. Even though the systems of DAEs in Definitions 19-20 in principle allow state-dependent manifolds such as $\operatorname{ker} \frac{\partial}{\partial \mathbf{x}^{\prime}} \mathbf{F}\left(\mathbf{d}^{\prime}(\mathbf{x}), \mathbf{x}, t\right)$ or $\operatorname{im} \frac{\partial}{\partial \mathbf{x}^{\prime}} \mathbf{F}\left(\mathbf{d}^{\prime}(\mathbf{x}), \mathbf{x}, t\right)$, this could lead to numerical difficulties. However, all the systems considered in this work do not have this dependency and can be described by a more structured differential equation like (3.29) fulfilling Assumption 12. Therefore, we do not further examine these complicated cases.

\subsection{Analysis of the Coupled System}

Having defined the three generalised elements, we can now study the DAE that is obtained from describing a circuit's behaviour containing these type of elements with modified nodal analysis. The structure and content of this section follows [55]. 


\subsubsection{Generalised elements in MNA}

We consider a circuit containing inductance-like, capacitance-like and resistance-like elements. As in Examples 2, 3 and 4 we have shown that classical (and flux-charge formulated) inductances, capacitances and resistances are described by our generalised element definitions, we can describe our circuit with the following system of DAEs

$$
\begin{aligned}
\mathbf{A}_{\varepsilon} \frac{\mathrm{d}}{\mathrm{d} t} \mathbf{q}_{\varepsilon}+\mathbf{A}_{\rho} \mathbf{i}_{\rho}+\mathbf{A}_{\mu} \mathbf{i}_{\mu}+\mathbf{A}_{\mathrm{V}} \mathbf{i}_{\mathrm{V}}+\mathbf{A}_{\mathrm{I}} \mathbf{i}_{\mathrm{s}}(t) & =0, \\
\mathbf{F}_{\mu}\left(\frac{\mathrm{d}}{\mathrm{d} t} \mathbf{d}_{\mu}\left(\mathbf{x}_{\mu}, \mathbf{i}_{\mu}\right), \mathbf{x}_{\mu}, \mathbf{i}_{\mu}, \mathbf{A}_{\mu}^{\top} \mathbf{e}, t\right) & =0, \\
\mathbf{F}_{\varepsilon}\left(\frac{\mathrm{d}}{\mathrm{d} t} \mathbf{d}_{\varepsilon}\left(\mathbf{x}_{\varepsilon}, \mathbf{A}_{\varepsilon}^{\top} \mathbf{e}\right), \mathbf{x}_{\varepsilon}, \frac{\mathrm{d}}{\mathrm{d} t} \mathbf{q}_{\varepsilon}, \mathbf{A}_{\varepsilon}^{\top} \mathbf{e}, t\right) & =0, \\
\mathbf{F}_{\rho}\left(\frac{\mathrm{d}}{\mathrm{d} t} \mathbf{d}_{\rho}\left(\mathbf{x}_{\rho}, \mathbf{i}_{\rho}, \mathbf{A}_{\rho}^{\top} \mathbf{e}\right), \mathbf{x}_{\rho}, \mathbf{i}_{\rho}, \mathbf{A}_{\rho}^{\top} \mathbf{e}, t\right) & =0, \\
\mathbf{A}_{\mathrm{V}}^{\top} \mathbf{e}-\mathbf{v}_{\mathrm{s}} & =0 .
\end{aligned}
$$

Here, the vector-valued functions $\mathbf{F}_{\mu}, \mathbf{F}_{\varepsilon}$ and $\mathbf{F}_{\rho}$ contain, for circuits with $n_{\mu}\left(n_{\varepsilon}\right.$ and $\left.n_{\rho}\right)$ inductance-like (capacitance-like and resistance-like) elements, on the $i$-th block-diagonal entry the DAE describing the $i$-th inductance-like (capacitance-like and resistance-like) element. Note that, inspired by the flux-charge formulation of MNA, the capacitance-like elements are embedded into Kirchhoff's current law through the charges $\mathbf{q}_{\varepsilon}$ rather than introducing the currents $\mathbf{i}_{\varepsilon}=\frac{\mathrm{d}}{\mathrm{d} t} \mathbf{q}_{\varepsilon}$ explicitly.

Whereas in MNA, the currents through branches containing resistances $\mathbf{i}_{\mathrm{R}}$ are directly replaced by their lumped element model in KCL (2.55a), in the MNA system containing the generalised elements (4.16), $\mathbf{i}_{\rho}$ is kept as a degree of freedom in (4.16a). In addition to this, in the conventional MNA (2.54), the charges of the capacitances $\mathbf{q}_{\varepsilon}$ are also replaced by their lumped voltage relation in (2.54a). However, in our system (4.16) the charges are kept as, a priori, the explicit charges-to-voltage relation of capacitance-like elements can be unkown. These structural differences in the system, however, do not imply changes in the analysis's results that follows. Therefore, if it is required and possible, the currents $\mathbf{i}_{\rho}$ and the charges $\mathbf{q}_{\varepsilon}$ can be replaced by their lumped element models in (4.16a).

\subsubsection{DAE index of the circuit}

To state the index results of the generalised elements MNA (4.16) in terms of the properties of some matrices, we define, like in [18], the projector $\mathbf{Q}_{\star}$ onto ker $\mathbf{A}_{\star}^{\top}$.

Theorem 1 (DAE index of the circuit). Consider a circuit containing inductance-like elements $(\mu)$, capacitancelike elements $(\varepsilon)$ coupled through charges $\left(\mathbf{q}_{\varepsilon}\right)$, resistance-like elements $(\rho)$ fulfilling Assumption 13 and voltage (V) and current (I) sources. Let the circuit be described with modified nodal analysis (4.16) and fulfil Assumption 10. Then, the system of differential algebraic equations (4.16) has differential index

(i) 1 or less, if there are no cutsets of only current sources and inductance-like elements (I $\mu$-cutsets), that is,

$$
\operatorname{ker}\left(\mathbf{A}_{\rho} \mathbf{A}_{\varepsilon} \mathbf{A}_{\mathrm{V}}\right)^{\top}=\{\mathbf{0}\}
$$

nor loops of only voltage sources and capacitance-like elements, with at least one voltage source (EV-loops), that is,

$$
\operatorname{ker} \mathbf{Q}_{\rho}^{\top} \mathbf{A}_{\mathrm{V}}=\{\mathbf{0}\}
$$


(ii) 2, otherwise.

Proof. The proof follows analogous to the differential index proof in [18] by considering resistance-like elements instead of resistances, capacitance-like elements accounting for the capacitances and inductance-like elements replacing the inductances.

Remark 19. If the capacitance-like elements are coupled to the circuit with the currents $\mathbf{i}_{\varepsilon}$ as degrees of freedom instead of the charges $\mathbf{q}_{\varepsilon}$, the system also has index 2 when containing loops of only capacitances. However, as mentioned before, the index results do not change depending on whether currents through resistance-like elements are part of the degrees of freedom or not.

This result is consistent with the index results of classic and flux-charge formulated MNA in [18], where it is stated that the systems have index 2 when the circuit contains LI-cutsets or CV-loops. In [55], the corresponding index results are presented for circuits of MNA containing also inductance-like elements. In [24] a more elaborate proof is given for the index of circuits containing even more general elements. The result coincides with the one obtained here.

Remark 20. Note that the classification of elements is unique in the sense that one element " $\star$ " fulfilling Assumption 13 can not be classified as two different generalised element types. Otherwise, contradictions could arise, as their would exist circuits where either their current ( $\mathrm{\star} \star$-cutset$)$ or voltage $(\star V$-loop) excitation would yield a DAE that is both index 1 and index 2 (see Theorem 1 ).

\subsubsection{Linearity of the index 2 components}

The system arising from classical as well as flux-charge formulated MNA has a special structure, as the index 2 components appear linearly in the original system [132]. This property eases the handling of the DAE even in the case of having index 2. Here, for example, consistent initialisation is facilitated [129, Chapter 2.3] and the numerical error that can occur due to the DAE's sensitivity towards perturbations is controllable, as it is not propagated in time [22]. The property can be expanded to circuits containing generalised elements, provided the inductance-like element has a special structure.

Proposition 2 (Linear index 2 components [55]). If in all the inductance-like elements contained in $\mu I$-cutsets, the voltage appears linearly, that is, they have the structure

$$
0=\mathbf{F}_{\mu}\left(\frac{\mathrm{d}}{\mathrm{d} t} \mathbf{d}_{\mu}\left(\mathbf{x}_{\mu}, \mathbf{i}_{\mu}\right), \mathbf{x}_{\mu}, \mathbf{i}_{\mu}, \mathbf{v}_{\mu}, t\right)=\tilde{\mathbf{F}}_{\mu}\left(\frac{\mathrm{d}}{\mathrm{d} t} \mathbf{d}_{\mu}\left(\mathbf{x}_{\mu}, \mathbf{i}_{\mu}\right), \mathbf{x}_{\mu}, \mathbf{i}_{\mu}, t\right)+\mathbf{B v}_{\mu},
$$

with a constant matrix $\mathbf{B} \in \mathbb{R}^{\left(n_{\mathrm{dof}}+n_{\mu}\right) \times n_{\mu}}$, then the index 2 components of the MNA DAE with refined models (4.16) for circuits fulfilling Assumption 10 appear always linearly in the system.

Proof. Following the differential index proof in [18] with the modifications for the refined models' system (4.16), it can be seen that the index 2 components, that is, the components that are defined by the hidden constraints, are the node potentials in $\rho \mathrm{I}$-cutsets and the currents through branches of voltage sources in $\varepsilon \mathrm{V}$-loops. Therefore, if $\mathbf{Q}_{\varepsilon \rho \mathrm{V}}$ is a projector onto $\operatorname{ker}\left(\mathbf{A}_{\varepsilon} \mathbf{A}_{\rho} \mathbf{A}_{\mathrm{V}}\right)^{\top}$ and $\overline{\mathbf{Q}}_{\mathrm{V}-\varepsilon}$ is a projector onto $\operatorname{ker} \mathbf{Q}_{\varepsilon}^{\top} \mathbf{A}_{\mathrm{V}}$, 
then $\mathbf{Q}_{\varepsilon \rho \mathrm{V}} \mathbf{e}$ and $\overline{\mathbf{Q}}_{\mathrm{V}-\varepsilon} \mathbf{i}_{\mathrm{V}}$ are the possible index 2 components. The only instances where those can potentially appear in the original DAE are in KCL (4.16a)

$$
\mathbf{A}_{\varepsilon} \frac{\mathrm{d}}{\mathrm{d} t} \mathbf{q}_{\varepsilon}+\mathbf{A}_{\rho} \mathbf{i}_{\rho}+\mathbf{A}_{\mu} \mathbf{i}_{\mu}+\mathbf{A}_{\mathrm{V}}\left(\overline{\mathbf{P}}_{\mathrm{V}-\varepsilon}+\overline{\mathbf{Q}}_{\mathrm{V}-\varepsilon}\right) \mathbf{i}_{\mathrm{V}}+\mathbf{A}_{\mathrm{I}} \mathbf{i}_{\mathrm{s}}(t)=0
$$

and in the equation describing the inductance-like element

$$
\mathbf{F}_{\mu}\left(\frac{\mathrm{d}}{\mathrm{d} t} \mathbf{d}_{\mu}\left(\mathbf{x}_{\mu}, \mathbf{i}_{\mu}\right), \mathbf{x}_{\mu}, \mathbf{i}_{\mu}, \mathbf{A}_{\mu}^{\top}\left(\mathbf{P}_{\varepsilon \rho \mathrm{V}}+\mathbf{Q}_{\varepsilon \rho \mathrm{V}}\right) \mathbf{e}, t\right)=0
$$

with complementary projectors $\mathbf{P}_{\varepsilon \rho \mathrm{V}}=\mathbf{I}-\mathbf{Q}_{\varepsilon \rho \mathrm{V}}$ and $\overline{\mathbf{P}}_{\mathrm{V}-\varepsilon}=\mathbf{I}-\overline{\mathbf{Q}}_{\mathrm{V}-\varepsilon}$. If in all inductance-like elements in $\mu \mathrm{I}$-cutsets, the voltage appears linearly, then (4.16b) can be rewritten as

$$
\begin{aligned}
0 & =\mathbf{F}_{\mu}\left(\frac{\mathrm{d}}{\mathrm{d} t} \mathbf{d}_{\mu}\left(\mathbf{x}_{\mu}, \mathbf{i}_{\mu}\right), \mathbf{x}_{\mu}, \mathbf{i}_{\mu}, \mathbf{A}_{\mu}^{\top}\left(\mathbf{P}_{\varepsilon \rho \mathrm{V}}+\mathbf{Q}_{\varepsilon \rho \mathrm{V}}\right) \mathbf{e}, t\right) \\
& =\tilde{\mathbf{F}}_{\mu}\left(\frac{\mathrm{d}}{\mathrm{d} t} \mathbf{d}_{\mu}\left(\mathbf{x}_{\mu}, \mathbf{i}_{\mu}\right), \mathbf{x}_{\mu}, \mathbf{i}_{\mu}, \mathbf{A}_{\mu}^{\top} \mathbf{P}_{\varepsilon \rho \mathrm{V}} \mathbf{e}, t\right)+\tilde{\mathbf{B}} \mathbf{Q}_{\varepsilon \rho \mathrm{V}} \mathbf{e}
\end{aligned}
$$

and thus all index 2 variables appear linearly in the original system.

Remark 21. The structure of the capacitance-like and resistance-like elements do not influence the linearity of the index 2 components as long as capacitance-like elements are coupled through the charges instead of the currents.

\subsection{Classification of field models}

In the following we present refined models that can be classified according to our generalised elements definitions. The examples and analysis follows [24], [55].

Remark 22. For the entire chapter and thus the results that are presented in it we consider Assumptions 1-8 for all domains and materials, as well as Assumption 11 for the discretisation, to be fulfilled.

\subsubsection{Discrete gauging}

Some systems of PDEs, such as for example the magnetoquasistatic curl-curl equation for the $\vec{A}^{*}$ formulation (2.20), are not uniquely solvable due to the gauging freedom. This property is inherited by their semidiscrete counterparts that, even with incorporated boundary and initial conditions, are not uniquely solvable. To deal with this impasse, discrete gauging can be performed.

The gauging conditions can be applied in different ways. For example in [20], [133], an additional equation describing the gauging condition is added to the original semidiscrete system of equations. Alternatively, in [83], a term is added to the stiffness matrix yielding a uniquely solvable system. Some iterative solvers, such as the conjugate gradient method, implicitly gauge the system and thus return a valid solution of the system of equations without the need of explicitly using a gauging condition [134]. Another alternative is the so-called tree-cotree gauge [135], [136]. This method allows gauging curl-curl equations by deleting the 
degrees of freedom that span divergence fields and thus keeping only unknowns that describe a divergencefree solution.

For a simply connected region $\Omega_{\mathrm{t}}$, a tree $T$ is constructed on the mesh and the values of the degrees of freedom that are on $T$ are set to zero. For multiply connected regions, cuts have to be defined on the domain $\Omega_{\mathrm{t}}$ to gauge correctly (see [98]). To incorporate tree-cotree gauging to our systems, we define a projector $\overline{\mathbf{P}}_{\mathrm{t}}$ that selects the remaining degrees of freedom. $\mathbf{P}_{\mathrm{t}}$ is a matrix with full column rank, corresponding to $\overline{\mathbf{P}}_{\mathrm{t}}$ by deleting all its linear dependent columns. For more details about tree-cotree gauging see [136].

\subsubsection{Inductance-like elements}

We present two different semidiscrete magnetoquasistatic field formulations that can be categorised as inductance-like elements. For the index analysis of $\vec{A}^{*}$ formulated field systems coupled to circuits, already known results from [22] are confirmed by our study (see [55]), however, with a different approach than the previous work. The index analysis of the field-circuit coupled system with $\vec{T}-\Omega$ formulation was first presented in [55]. Related works, such as [21] do not consider fully three dimensional field problems nor give the index results of the coupled system. The structure and content of this part follow [55].

Before presenting the analysis of both formulations, we state a preliminary result that eases the later study.

Proposition 3 (Inductance-like element [55]). An element which is described by the DAE

$$
\mathbf{F}_{\mu}\left(\frac{\mathrm{d}}{\mathrm{d} t} \mathbf{d}_{\mu}\left(\mathbf{x}_{\mu}, \mathbf{i}_{\mu}\right), \mathbf{x}_{\mu}, \mathbf{i}_{\mu}, \mathbf{v}_{\mu}, t\right)=0
$$

where, after at most one time differentiation of its original equation

$$
\frac{\mathrm{d}}{\mathrm{d} t} \mathbf{F}_{\mu}\left(\frac{\mathrm{d}}{\mathrm{d} t} \mathbf{d}_{\mu}\left(\mathbf{x}_{\mu}, \mathbf{i}_{\mu}\right), \mathbf{x}_{\mu}, \mathbf{i}_{\mu}, \mathbf{v}_{\mu}, t\right)=0
$$

and using only algebraic manipulations of (4.17) and (4.18), a system with the structure

$$
\begin{aligned}
\frac{\mathrm{d}}{\mathrm{d} t} \mathbf{x}_{\mu} & =\mathbf{f}_{\mathbf{x}}\left(\mathbf{x}_{\mu}, \mathbf{i}_{\mu}, \mathbf{v}_{\mu}, t\right) \\
\frac{\mathrm{d}}{\mathrm{d} t} \mathbf{i}_{\mu} & =\mathbf{L}_{\mu}\left(\mathbf{i}_{\mu}, \mathbf{x}_{\mu}\right)^{-1} \mathbf{v}_{\mu}+\mathbf{f}_{\mathbf{i}}\left(\mathbf{x}_{\mu}, \mathbf{i}_{\mu}, t\right)
\end{aligned}
$$

can be obtained with positive definite $\mathbf{L}_{\mu}\left(\mathbf{i}_{\mu}, \mathbf{x}_{\mu}\right)$, is an inductance-like device.

Proof. Equation (4.19) has already the same structure as (4.3) and, by defining an antiderivative of $\mathbf{L}_{\mu}\left(\mathbf{i}_{\mu}, \mathbf{x}_{\mu}\right)$ as $\phi_{\mu}\left(\mathbf{i}_{\mu}, \mathbf{x}_{\mu}, t\right):=\int_{\mathbf{i}_{\mu, 0}}^{\mathbf{i}_{\mu}} \mathbf{L}_{\mu}\left(\boldsymbol{\xi}, \mathbf{x}_{\mu}\right) \mathrm{d} \boldsymbol{\xi}$, where $\mathbf{i}_{\mu, 0}$ is the initial value of $\mathbf{i}_{\mu}$, equation (4.4) can be reconstructed with

$$
\begin{aligned}
\mathbf{f}_{\phi}\left(\mathbf{x}_{\mu}, \mathbf{i}_{\mu}, \mathbf{v}_{\mu}, t\right)= & \mathbf{v}_{\mu}+\frac{\partial}{\partial \mathbf{x}_{\mu}}\left(\phi_{\mu}\left(\mathbf{i}_{\mu}, \mathbf{x}_{\mu}, t\right)\right) \mathbf{f}_{\mathbf{x}}\left(\mathbf{x}_{\mu}, \mathbf{i}_{\mu}, \mathbf{v}_{\mu}, t\right) \\
& +\mathbf{L}_{\mu}\left(\mathbf{i}_{\mu}, \mathbf{x}_{\mu}\right) \mathbf{f}_{\mathbf{i}}\left(\mathbf{x}_{\mu}, \mathbf{i}_{\mu}, t\right)
\end{aligned}
$$

Property (i) of Definition 19 is fulfilled, as

$$
\frac{\partial}{\partial \mathbf{i}_{\mu}} \phi\left(\mathbf{i}_{\mu}, \mathbf{x}_{\mu}, t\right)=\mathbf{L}_{\mu}\left(\mathbf{i}_{\mu}, \mathbf{x}_{\mu}\right),
$$


is positive definite and thus non-singular and Property (ii) follows from

$$
\frac{\partial}{\partial \mathbf{v}_{\mu}}\left(\left(\frac{\partial \phi}{\partial \mathbf{i}_{\mu}}\right)^{-1}\left(-\frac{\partial \phi}{\partial \mathbf{x}_{\mu}} \mathbf{f}_{\mathbf{x}}-\frac{\partial \phi}{\partial t}+\mathbf{f}_{\phi}\right)\right)=\mathbf{L}_{\mu}^{-1}\left(\mathbf{i}_{\mu}, \mathbf{x}_{\mu}\right)
$$

being positive definite.

\section{$\overrightarrow{A^{*}}$ magnetoquasistatic formulation}

For simplicity of notation, we first consider only field independent materials. After spatial discretisation of the eddy current PDE with the $\vec{A}^{*}$ formulation (2.20) together with its circuit coupling equations (2.29) and (2.30), we obtain the semidiscrete system of DAEs

$$
\begin{aligned}
\mathbf{M}_{\sigma} \frac{\mathrm{d}}{\mathrm{d} t} \mathbf{a}+\mathbf{C}^{\top} \mathbf{M}_{\nu} \mathbf{C a}-\mathbf{X}_{\mathrm{s}} \mathbf{i}_{\mu} & =0 \\
\frac{\mathrm{d}}{\mathrm{d} t} \mathbf{X}_{\mathrm{s}}^{\top} \mathbf{a}-\mathbf{v}_{\mu} & =0 .
\end{aligned}
$$

Here, $\mathbf{X}_{\mathrm{s}}$ is the spatial discretisation of the winding density function $\chi_{\mathrm{s}}$, where the $k$-th column represents the discrete vector of the $k$-th winding function $\vec{\chi}_{\mathrm{k}}$. The lumped parameters $\mathbf{i}_{\mu}$ and $\mathbf{v}_{\mu}$ are the vectors of currents and voltages, respectively.

The gauging freedom of the curl-curl equation in the non-conducting region $\Omega_{\mathrm{c}}^{\mathrm{c}}$ is inherited by its spatially discretised counterpart. Therefore, a gauge condition is required to ensure a uniquely solvable system.

Assumption 14 (Gauged $\vec{A}^{*}$ formulation [55]). We assume the DAE (4.21) is gauged with a tree-cotree gauging and thus rewrite it as

$$
\begin{aligned}
\overline{\mathbf{M}}_{\sigma} \frac{\mathrm{d}}{\mathrm{d} t} \mathbf{a}_{\mathrm{red}}+\overline{\mathbf{K}}_{\nu} \mathbf{a}_{\mathrm{red}}-\overline{\mathbf{X}}_{\mathrm{s}} \mathbf{i}_{\mu}=0 \\
\frac{\mathrm{d}}{\mathrm{d} t} \overline{\mathbf{X}}_{\mathrm{s}}^{\top} \mathbf{a}_{\mathrm{red}}-\mathbf{v}_{\mu}=0,
\end{aligned}
$$

where $\overline{\mathbf{M}}_{\sigma}=\mathbf{P}_{\mathrm{t}}^{\top} \mathbf{M}_{\sigma} \mathbf{P}_{\mathrm{t}}, \overline{\mathbf{K}}_{\nu}=\mathbf{P}_{\mathrm{t}}^{\top} \mathbf{C}^{\top} \mathbf{M}_{\nu} \mathbf{C} \mathbf{P}_{\mathrm{t}}$ and $\overline{\mathbf{X}}_{\mathrm{s}}=\mathbf{P}_{\mathrm{t}}^{\top} \mathbf{X}_{\mathrm{s}}$, and such that the matrix pencil $\lambda \overline{\mathbf{M}}_{\sigma}+\overline{\mathbf{K}}_{\nu}$ with $\lambda \in \mathbb{R}$ is regular.

Assumption 15 (Discrete winding function [55]). The semidiscrete and gauged winding function $\overline{\mathbf{X}}_{\mathrm{s}}$ is assumed to have the following properties

(i) $\overline{\mathbf{X}}_{\mathrm{s}}$ has full column rank.

(ii) $\operatorname{im} \overline{\mathbf{X}}_{\mathrm{s}} \perp \operatorname{im} \overline{\mathbf{M}}_{\sigma}$.

The last assumption translates properties of the continuous winding functions and their domains, presented in Assumption 6, into properties of their discrete winding matrices. Property $(i)$ is inspired by the fact that different columns of $\overline{\mathbf{X}}_{\mathrm{s}}$ are discretisations of different winding functions $\vec{\chi}_{\mathrm{s}}^{(j)}$ that, according to Assumption 6 , have disjoint support. Also, the conducting domain $\Omega_{\mathrm{c}}$ and the source domain $\Omega_{\mathrm{s}}$ are disjoint, which leads to Property (ii). This could be relaxed, in case the two domains might touch each other but is kept for simplicity. 
Proposition 4 ( $\vec{A}^{*}$ inductance-like element [55]). Provided Assumptions 14 and 15 are fulfilled, then the semidiscrete gauged system of DAEs arising from the curl-curl $\vec{A}^{*}$ formulation together with its circuit coupling equation (4.22) forms an inductance-like element.

Proof. We start by defining a projector $\mathbf{Q}_{\sigma}$ onto ker $\overline{\mathbf{M}}_{\sigma}$ and its complementary $\mathbf{P}_{\sigma}=\mathbf{I}-\mathbf{Q}_{\sigma}$, to split system (4.22) onto

$$
\begin{aligned}
\overline{\mathbf{M}}_{\sigma} \frac{\mathrm{d}}{\mathrm{d} t} \mathbf{a}_{\mathrm{red}}+\mathbf{P}_{\sigma}^{\top} \overline{\mathbf{K}}_{\nu} \mathbf{a}_{\mathrm{red}}-\mathbf{P}_{\sigma}^{\top} \overline{\mathbf{X}}_{\mathrm{s}} \mathbf{i}_{\mu}=0 \\
\mathbf{Q}_{\sigma}^{\top} \overline{\mathbf{K}}_{\nu} \mathbf{a}_{\mathrm{red}}-\mathbf{Q}_{\sigma}^{\top} \overline{\mathbf{X}}_{\mathrm{s}} \mathbf{i}_{\mu}=0 \\
\frac{\mathrm{d}}{\mathrm{d} t} \overline{\mathbf{X}}_{\mathrm{s}}^{\top} \mathbf{a}_{\mathrm{red}}-\mathbf{v}_{\mu}=0
\end{aligned}
$$

Due to the definition of the projection matrices, and using the fact that $\overline{\mathbf{M}}_{\sigma}$ is symmetric, $\overline{\mathbf{M}}_{\sigma}+\mathbf{Q}_{\sigma}^{\top} \mathbf{Q}_{\sigma}$ is positive definite and thus invertible. Multiplication of the first equation (4.23a) by $\left(\overline{\mathbf{M}}_{\sigma}+\mathbf{Q}_{\sigma}^{\top} \mathbf{Q}_{\sigma}\right)^{-1}$ yields an ODE for $\mathbf{P}_{\sigma} \frac{\mathrm{d}}{\mathrm{d} t} \mathbf{a}_{\text {red }}$

$$
\mathbf{P}_{\sigma} \frac{\mathrm{d}}{\mathrm{d} t} \mathbf{a}_{\mathrm{red}}=\mathbf{f}_{\sigma}\left(\mathbf{a}_{\mathrm{red}}, \mathbf{i}_{\mu}\right) .
$$

Using Assumption 14, $\mathbf{Q}_{\sigma}^{\top} \overline{\mathbf{K}}_{\nu} \mathbf{Q}_{\sigma}+\mathbf{P}_{\sigma}^{\top} \mathbf{P}_{\sigma}$ is positive definite. After one time differentiation of (4.23b), its multiplication by $\left(\mathbf{Q}_{\sigma}^{\top} \overline{\mathbf{K}}_{\nu} \mathbf{Q}_{\sigma}+\mathbf{P}_{\sigma}^{\top} \mathbf{P}_{\sigma}\right)^{-1}$, and the insertion of $\mathbf{f}_{\sigma}\left(\mathbf{a}_{\mathrm{red}}, \mathbf{i}_{\mu}\right)$, the following expression is obtained

$$
\mathbf{Q}_{\sigma} \frac{\mathrm{d}}{\mathrm{d} t} \mathbf{a}_{\mathrm{red}}=\left(\mathbf{Q}_{\sigma}^{\top} \overline{\mathbf{K}}_{\nu} \mathbf{Q}_{\sigma}+\mathbf{P}_{\sigma}^{\top} \mathbf{P}_{\sigma}\right)^{-1}\left(\mathbf{Q}_{\sigma}^{\top} \mathbf{f}_{\sigma}\left(\mathbf{a}_{\mathrm{red}}, \mathbf{i}_{\mu}\right)-\mathbf{Q}_{\sigma}^{\top} \overline{\mathbf{X}}_{\mathrm{s}} \frac{\mathrm{d}}{\mathrm{d} t} \mathbf{i}_{\mu}\right) .
$$

The equation for $\left(\mathbf{P}_{\sigma}+\mathbf{Q}_{\sigma}\right) \frac{\mathrm{d}}{\mathrm{d} t} \mathbf{a}_{\text {red }}$ is then inserted into (4.23c), which leads to

$$
\frac{\mathrm{d}}{\mathrm{d} t} \mathbf{i}_{\mu}=\mathbf{L}_{\mu}^{-1} \mathbf{v}_{\mathrm{s}}+\mathbf{f}_{\mathrm{s}}\left(\mathbf{a}_{\mathrm{red}}, \mathbf{i}_{\mathrm{s}}\right),
$$

with $\mathbf{L}_{\mu}=\overline{\mathbf{X}}_{\mathrm{s}}^{\top} \mathbf{Q}_{\sigma}\left(\mathbf{Q}_{\sigma}^{\top} \overline{\mathbf{K}}_{\nu} \mathbf{Q}_{\sigma}+\mathbf{P}_{\sigma}^{\top} \mathbf{P}_{\sigma}\right)^{-1} \mathbf{Q}_{\sigma}^{\top} \overline{\mathbf{X}}_{\mathrm{s}}$. This can be used in (4.24) to obtain an ODE for $\mathbf{Q}_{\sigma} \frac{\mathrm{d}}{\mathrm{d} t} \mathbf{a}_{\text {red }}$

$$
\mathbf{Q}_{\sigma} \frac{\mathrm{d}}{\mathrm{d} t} \mathbf{a}_{\mathrm{red}}=\mathbf{f}_{0}\left(\mathbf{a}_{\mathrm{red}}, \mathbf{i}_{\mu}, \mathbf{v}_{\mu}\right) \text {. }
$$

Combining both the expressions for $\mathbf{Q}_{\sigma} \frac{\mathrm{d}}{\mathrm{d} t} \mathbf{a}_{\text {red }}$ and $\mathbf{P}_{\sigma} \frac{\mathrm{d}}{\mathrm{d} t} \mathbf{a}_{\text {red }}$ leads to an ODE like (4.19) for $\mathbf{x}_{\mu}=\mathbf{a}_{\text {red }}$.

Using Assumption 15, $\mathbf{Q}_{\sigma}^{\top} \overline{\mathbf{X}}_{\mathrm{s}}$ has full column rank and therefore $\mathbf{L}_{\mu}$ is positive definite. Thus (4.25) is an expression of the type (4.20) which, according to Proposition 3 means that it has the structure of an inductance-like element.

The last proposition, together with Theorem 1 proofs that the $\vec{A}^{*}$ curl-curl equation coupled to a circuit influences behaves like inductances from the index point of view. The special case of the excitation of the field system either with a voltage or a current source is a particular circuit and the following Corollary is immediately deduced.

Corollary 1 (Excitation index of the $\vec{A}^{*}$ formulation [55]). Let Assumptions 14 and 15 hold, then the semidiscrete gauged system of equations of the $\vec{A}^{*}$ formulation with circuit coupling equation (4.22) has differential index

- 1 , if the voltage $\mathbf{v}_{\mu}$ is given as a function of time.

- 2 , if the current $\mathbf{i}_{\mu}$ is given as a function of time. 
Combining Proposition 4 and Theorem 1 leads to the same index result as the previous work in [22].

Remark 23. In [20] it is shown that different gauge conditions can change the index of a semidiscrete field system for the specific case of full Maxwell's equations with the A- $\phi$ formulation. However, in our case, even though the analysis is performed for eddy current problems with a tree-cotree gauging condition, the same results follow analogously for other types of gauges, as long as the gauging condition still ensures Assumption 15 is fulfilled. This holds in particular for the gauging condition considered in [22].

\section{$\vec{T}-\Omega$ magnetoquasistatic formulation}

Again we start by analysing the $\vec{T}-\Omega$ formulated magnetoquasistatic DAE for materials that are only spacedependent. After a space discretisation method is applied to the $\vec{T}-\Omega$ formulation's PDE (2.24) and their circuit coupling equations (2.31) and (2.33), the following semidiscrete system of DAEs

$$
\begin{array}{r}
\mathbf{C}^{\top} \mathbf{M}_{\rho} \mathbf{C t}+\mathbf{M}_{\mu} \frac{\mathrm{d}}{\mathrm{d} t} \mathbf{t}+\widetilde{\mathbf{S}}^{\top} \frac{\mathrm{d}}{\mathrm{d} t} \boldsymbol{\Psi}+\mathbf{Y}_{\mathrm{s}} \frac{\mathrm{d}}{\mathrm{d} t} \mathbf{i}_{\mu}=0 \\
\widetilde{\mathbf{S}} \mathbf{M}_{\mu}\left(\mathbf{t}+\widetilde{\mathbf{S}}^{\top} \boldsymbol{\Psi}+\mathbf{Y}_{\mathrm{s}} \mathbf{i}_{\mu}\right)=0 \\
\mathbf{Y}_{\mathrm{s}}^{\top} \mathbf{M}_{\mu}\left(\frac{\mathrm{d}}{\mathrm{d} t} \mathbf{t}+\widetilde{\mathbf{S}}^{\top} \frac{\mathrm{d}}{\mathrm{d} t} \boldsymbol{\Psi}+\mathbf{Y}_{\mathrm{s}} \frac{\mathrm{d}}{\mathrm{d} t} \mathbf{i}_{\mu}\right)-\mathbf{v}_{\mu}=0,
\end{array}
$$

is obtained. Here, the matrix $\mathbf{Y}_{\mathrm{s}}$ represents the spatial discretisation of the winding function for the source magnetic field strength (2.31).

Remark 24. As it can be visualised in Figure 2.3, the (discrete) magnetic vector potential a and the (discrete) electric vector potential $\mathbf{t}$ are dual quantities. Therefore, in FIT, if $\mathbf{a}$ is defined at the primal mesh and thus the primal curl operator $\mathbf{C}$ is applied to it, $\mathbf{t}$ should be defined at the dual mesh and the dual curl operator $\widetilde{\mathbf{C}}=\mathbf{C}^{\top}$ should be used. This is important to be made consistently for a correct coupling or usage of both formulations.

Primal and dual space form a one-to-one relation between each other, as the primal space is the dual space of the dual. Therefore, as long as within one problem the duality between the degrees of freedom is chosen consistently, $G$ or $\widetilde{G}$ can both be correctly used as primal mesh. Therefore, for the analysis of the systems as well as the separate simulation of them, only the internal quantities of each system, e.g. $t$ and $\Psi$ in the $\vec{T}-\Omega$ case, are to be located consistently. For simplicity of notation and implementation (e.g. the re-usage of matrices) we use the primal curl operator $\mathbf{C}$ for its application to the semidiscrete electric vector potential $\mathrm{t}$ and choose the rest consistently.

Again a gauging freedom arises for the electric vector potential on the conducting region $\Omega_{\mathrm{c}}$ where $\mathbf{t}$ is nonzero. Note that the matrix $\mathbf{P}_{\mathrm{t}}$ applyed for the tree-cotree gauging to the degrees of freedom $\mathbf{t}$ in the following assumption is not the same than the one of the semidiscrete $\vec{A}^{*}$ formulation (4.22).

Assumption 16 (Gauged $\vec{T}-\Omega$ formulation [55]). We assume the semidiscrete $\vec{T}-\Omega$ system (2.24) is gauged 
with a tree-cotree gauge and thus consider the DAE

$$
\begin{aligned}
& \mathbf{P}_{\mathrm{t}}^{\top} \mathbf{C}^{\top} \mathbf{M}_{\rho} \mathbf{C} \mathbf{P}_{\mathrm{t}} \mathbf{t}_{\mathrm{red}}+\mathbf{P}_{\mathrm{t}}^{\top} \mathbf{M}_{\mu}\left(\mathbf{P}_{\mathrm{t}} \frac{\mathrm{d}}{\mathrm{d} t} \mathbf{t}_{\mathrm{red}}+\widetilde{\mathbf{S}}^{\top} \frac{\mathrm{d}}{\mathrm{d} t} \boldsymbol{\Psi}+\mathbf{Y}_{\mathrm{s}} \frac{\mathrm{d}}{\mathrm{d} t} \mathbf{i}_{\mu}\right)=0 \\
& \widetilde{\mathbf{S}} \mathbf{M}_{\mu}\left(\mathbf{P}_{\mathrm{t}} \mathbf{t}_{\mathrm{red}}+\widetilde{\mathbf{S}}^{\top} \boldsymbol{\Psi}+\mathbf{Y}_{\mathrm{s}} \mathbf{i}_{\mu}\right)=0 \\
& \mathbf{Y}_{\mathrm{s}}^{\top} \mathbf{M}_{\mu}\left(\mathbf{P}_{\mathrm{t}} \frac{\mathrm{d}}{\mathrm{d} t} \mathbf{t}_{\mathrm{red}}+\widetilde{\mathbf{S}}^{\top} \frac{\mathrm{d}}{\mathrm{d} t} \boldsymbol{\Psi}+\mathbf{Y}_{\mathrm{s}} \frac{\mathrm{d}}{\mathrm{d} t} \mathbf{i}_{\mu}\right)-\mathbf{v}_{\mu}=0,
\end{aligned}
$$

where the matrix $\mathbf{K}_{\rho}=\mathbf{P}_{\mathrm{t}}^{\top} \mathbf{C}^{\top} \mathbf{M}_{\rho} \mathbf{C P}_{\mathrm{t}}$ is positive definite and thus $\operatorname{det}\left(\mathbf{K}_{\rho}\right) \neq 0$.

Proposition 5 (Gradient free gauging [55]). The semidiscrete, gauged field $\mathbf{P}_{\mathrm{t}} \mathbf{x}$ can not be represented by a gradient field, that is, for $\mathbf{x}, \mathbf{y} \neq \mathbf{0}$,

$$
\mathbf{P}_{\mathrm{t}} \mathbf{x} \neq \widetilde{\mathbf{S}}^{\top} \mathbf{y}
$$

Proof. The proposition follows from Assumption 16, that is, the gauging property of the truncated projector matrix $\mathbf{P}_{\mathrm{t}}$, and Lemma 1(a).

Proposition 6 (Discrete Helmholtz split [55]). For all $\mathbf{x} \in \mathbb{R}^{n}$, there exist $\mathbf{x}_{1} \in \mathbb{R}^{m}, \mathbf{x}_{2} \in \mathbb{R}^{n}$, such that

$$
\mathbf{x}=\widetilde{\mathbf{S}}^{\top} \mathbf{x}_{1}+\mathbf{M}_{\mu}^{-1} \mathbf{C}^{\top} \mathbf{x}_{2},
$$

with $n, m \in \mathbb{R}$ such that $\widetilde{\mathbf{S}}^{\top} \in \mathbb{R}^{n \times m}$ and $\mathbf{C} \in \mathbb{R}^{n \times n}$.

Proof. Using Lemma $1(a)$ and the positive definiteness of $\mathbf{M}_{\mu}, \operatorname{im}\left(\mathbf{M}_{\mu}^{-\frac{1}{2}} \mathbf{C}\right) \subseteq \operatorname{ker}\left(\widetilde{\mathbf{S}} \mathbf{M}_{\mu}^{\frac{1}{2}}\right)$. Furthermore, as, due to Lemma $1(b), \operatorname{dim} \operatorname{ker}\left(\widetilde{\mathbf{S}} \mathbf{M}_{\mu}^{\frac{1}{2}}\right)=\operatorname{rank}\left(\mathbf{M}_{\mu}^{-\frac{1}{2}} \mathbf{C}\right)$,

$$
\operatorname{ker}\left(\widetilde{\mathbf{S}} \mathbf{M}_{\mu}^{\frac{1}{2}}\right)=\operatorname{im}\left(\mathbf{M}_{\mu}^{-\frac{1}{2}} \mathbf{C}^{\top}\right) .
$$

Terefore, for all $\mathbf{y} \in \mathbb{R}^{n}$, there exist $\mathbf{y}_{1} \in \mathbb{R}^{m}$ and $\mathbf{y}_{2} \in \mathbb{R}^{n}$, such that

$$
\mathbf{y}=\mathbf{M}_{\mu}^{\frac{1}{2}} \widetilde{\mathbf{S}}^{\top} \mathbf{y}_{1}+\mathbf{M}_{\mu}^{-\frac{1}{2}} \mathbf{C}^{\top} \mathbf{y}_{2}
$$

As $\mathbf{M}_{\mu}^{-\frac{1}{2}}$ is positive definite, there exists an $\mathbf{y}_{0} \in \mathbb{R}^{n}$ with $\mathbf{x}=\mathbf{M}_{\mu}^{-\frac{1}{2}} \mathbf{y}_{0}=\widetilde{\mathbf{S}}^{\top} \mathbf{x}_{1}+\mathbf{M}_{\mu}^{-1} \mathbf{C}^{\top} \mathbf{x}_{2}$.

Assumption 17 (Discrete current densities [55]). For the spatially discrete winding density function of the $\vec{T}-\Omega$ formulation $\mathbf{Y}_{\mathrm{s}}$ and $\mathbf{P}_{\mathrm{t}}$ the tree-cotree gauging matrix,

$$
\mathbf{0} \neq \mathbf{C} \mathbf{Y}_{\mathrm{s}} \mathbf{x} \neq \mathbf{C P}_{\mathrm{t}} \mathbf{y}, \text { for } \mathbf{x}, \mathbf{y} \neq \mathbf{0} \text {. }
$$

Note that the previous assumption forces the semidiscrete source current density, which is built by applying the curl to the source magnetic field density

$$
\mathbf{j}_{\mathrm{s}}=\mathbf{C h}_{\mathrm{s}}=\mathrm{CY}_{\mathrm{s}} \mathbf{x}
$$

to be different from the conduction current density that arises from the application of the curl operator to the electric vector potential

$$
\mathbf{j}_{\mathrm{c}}=\mathbf{C P}_{\mathrm{t}} \mathbf{y} \text {. }
$$

This holds, as $\mathbf{j}_{\mathrm{s}}$ has only nonzero support in the (stranded) source domain $\Omega_{\mathrm{s}}$, whereas the conduction current density $\mathbf{j}_{\mathrm{c}}$ is only nonzero in $\Omega_{\mathrm{c}}$ and both domains are disjoint due to Assumption 6 . Furthermore, both matrices are constructed to have full column rank and thus are $\neq 0$ for $\mathbf{x}, \mathbf{y} \neq 0$. 
Proposition $7(\vec{T}-\Omega$ inductance-like element [55]). Given Assumptions 16 and 17 are fulfilled, then the semidiscrete, gauged system of DAEs arising from the $\vec{T}-\Omega$ formulation together with its circuit coupling equation (4.27) is an inductance-like element.

Proof. The first step is to find, with at most one time differentiation of the original problem, an ODE expression for the internal degrees of freedom $\mathbf{x}_{\mu}^{\top}=\left(\mathbf{t}_{\text {red }}^{\top} \boldsymbol{\Psi}^{\top}\right)$ depending on $\mathbf{x}_{\mu}, \mathbf{i}_{\mu}$ and $\mathbf{v}_{\mu}$ (see equation (4.19)).

One time differentation of equation (4.27b) allows to obtain, by inverting the positive definite matrix $\mathbf{L}_{\mu}=$ $\widetilde{\mathbf{S}} \mathbf{M}_{\mu} \widetilde{\mathbf{S}}^{\top}$ (see Assumption 11 and Lemmas 1 and 2),

$$
\frac{\mathrm{d}}{\mathrm{d} t} \mathbf{\Psi}=-\mathbf{L}_{\mu}^{-1} \widetilde{\mathbf{S}} \mathbf{M}_{\mu} \mathbf{P}_{\mathrm{t}} \frac{\mathrm{d}}{\mathrm{d} t} \mathbf{t}_{\mathrm{red}}-\mathbf{L}_{\mu}^{-1} \widetilde{\mathbf{S}} \mathbf{M}_{\mu} \mathbf{Y}_{\mathrm{s}} \frac{\mathrm{d}}{\mathrm{d} t} \mathbf{i}_{\mu} .
$$

In the following, an expression for $\frac{\mathrm{d}}{\mathrm{d} t} \mathbf{t}_{\text {red }}$ is to be found from (4.27a). For that, (4.28) is inserted into (4.27a), which yields

$$
\left(\mathbf{P}_{\mathrm{t}}^{\top} \mathbf{W} \mathbf{P}_{\mathrm{t}}\right) \frac{\mathrm{d}}{\mathrm{d} t} \mathbf{t}_{\mathrm{red}}=-\mathbf{P}_{\mathrm{t}}^{\top} \mathbf{W} \mathbf{Y}_{\mathrm{s}} \frac{\mathrm{d}}{\mathrm{d} t} \mathbf{i}_{\mu}-\mathbf{P}_{\mathrm{t}}^{\top} \mathbf{K}_{\rho} \mathbf{P}_{\mathrm{t}} \mathbf{t}_{\mathrm{red}},
$$

where $\mathbf{W}=\mathbf{M}_{\mu}-\mathbf{M}_{\mu} \widetilde{\mathbf{S}}^{\top} \mathbf{L}_{\mu}^{-1} \widetilde{\mathbf{S}} \mathbf{M}_{\mu}$. Now, the positive definiteness of $\left(\mathbf{P}_{\mathrm{t}}^{\top} \mathbf{W} \mathbf{P}_{\mathrm{t}}\right)$ is shown by rewriting $\mathbf{W}$ as

$$
\mathbf{W}=\mathbf{M}_{\mu}^{\frac{1}{2}} \mathbf{Q}_{\mu} \mathbf{M}_{\mu}^{\frac{1}{2}}
$$

with the symmetric projector and therefore positive semidefinite matrix $\mathbf{Q}_{\mu}=\left(\mathbf{I}-\mathbf{M}_{\mu}^{\frac{1}{2}} \widetilde{\mathbf{S}}^{\top} \mathbf{L}_{\mu}^{-1} \widetilde{\mathbf{S}} \mathbf{M}_{\mu}^{\frac{1}{2}}\right)$. Assuming there exists $\mathbf{x}$ such that $\mathbf{x}^{\top} \mathbf{P}_{\mathrm{t}}^{\top} \mathbf{W} \mathbf{P}_{\mathrm{t}} \mathbf{x}=0$, then, as $\mathbf{W}$ is positive semidefinite, $\mathbf{W}^{\frac{1}{2}} \mathbf{P}_{\mathrm{t}} \mathbf{x}=\mathbf{0}$, which implies $\mathbf{W} \mathbf{P}_{\mathrm{t}} \mathbf{x}=\mathbf{0}$. This, however, due to the definition of $\mathbf{W}$ leads to the equality $\mathbf{P}_{\mathrm{t}} \mathbf{x}=\widetilde{\mathbf{S}}^{\top} \mathbf{L}_{\mu}^{-1} \widetilde{\mathbf{S}} \mathbf{M}_{\mu} \mathbf{P}_{\mathrm{t}} \mathbf{x}$, which due to Proposition 5 can only hold for $\mathbf{x}=0$. Therefore, $\left(\mathbf{P}_{\mathrm{t}}^{\top} \mathbf{W} \mathbf{P}_{\mathrm{t}}\right)$ is positive definite and thus can be inverted in the expression (4.29) to obtain

$$
\frac{\mathrm{d}}{\mathrm{d} t} \mathbf{t}_{\mathrm{red}}=-\left(\mathbf{P}_{\mathrm{t}}^{\top} \mathbf{W} \mathbf{P}_{\mathrm{t}}\right)^{-1} \mathbf{P}_{\mathrm{t}}^{\top} \mathbf{W} \mathbf{Y}_{\mathrm{s}} \frac{\mathrm{d}}{\mathrm{d} t} \mathbf{i}_{\mu}-\left(\mathbf{P}_{\mathrm{t}}^{\top} \mathbf{W} \mathbf{P}_{\mathrm{t}}\right)^{-1} \mathbf{P}_{\mathrm{t}}^{\top} \mathbf{K}_{\rho} \mathbf{P}_{\mathrm{t}} \mathbf{t}_{\mathrm{red}} .
$$

Combining the expressions for $\frac{\mathrm{d}}{\mathrm{d} t} \boldsymbol{\Psi}$ (4.28) and $\frac{\mathrm{d}}{\mathrm{d} t} \mathbf{t}_{\mathrm{red}}$ (4.30) with the circuit coupling equation (4.23c) yields

$$
\mathbf{v}_{\mu}=\mathbf{L}_{\mu} \frac{\mathrm{d}}{\mathrm{d} t} \mathbf{i}_{\mu}-\mathbf{Y}_{\mathrm{s}}^{\top} \mathbf{W} \mathbf{P}_{\mathrm{t}}\left(\mathbf{P}_{\mathrm{t}}^{\top} \mathbf{W} \mathbf{P}_{\mathrm{t}}\right)^{-1} \mathbf{K}_{\rho} \mathbf{t}_{\mathrm{red}}
$$

where $\mathbf{L}_{\mu}=\mathbf{Y}_{\mathrm{s}}^{\top} \mathbf{W}_{\mathrm{P}} \mathbf{Y}_{\mathrm{s}}$, with $\mathbf{W}_{\mathrm{P}}=\left(\mathbf{W}-\mathbf{W} \mathbf{P}_{\mathrm{t}}\left(\mathbf{P}_{\mathrm{t}}^{\top} \mathbf{W} \mathbf{P}_{\mathrm{t}}\right)^{-1} \mathbf{P}_{\mathrm{t}}^{\top} \mathbf{W}\right)$. The last equation allows to extract an expression for $\frac{\mathrm{d}}{\mathrm{d} t} \mathbf{i}_{\mu}$, if $\mathbf{L}_{\mu}$ is shown to be positive definite. Let us verify this analogously to $\mathbf{P}_{\mathrm{t}}^{\top} \mathbf{W} \mathbf{P}_{\mathrm{t}}$ : Again we can write $\mathbf{W}_{\mathrm{P}}=\mathbf{W}^{\frac{1}{2}} \mathbf{Q}_{\mathrm{W}} \mathbf{W}^{\frac{1}{2}}$, with the symmetric projector $\mathbf{Q}_{\mathrm{W}}$. Similarly to the previous case, showing that $\left(\mathbf{W}-\mathbf{W} \mathbf{P}_{\mathrm{t}}\left(\mathbf{P}_{\mathrm{t}}^{\top} \mathbf{W} \mathbf{P}_{\mathrm{t}}\right)^{-1} \mathbf{P}_{\mathrm{t}}^{\top} \mathbf{W}\right) \mathbf{Y}_{\mathrm{s}} \mathbf{x}=\mathbf{0}$ only for $\mathbf{x}=0$ implies the positive definiteness. For $\mathbf{x}$ such that $\left(\mathbf{W}-\mathbf{W} \mathbf{P}_{\mathrm{t}}\left(\mathbf{P}_{\mathrm{t}}^{\top} \mathbf{W} \mathbf{P}_{\mathrm{t}}\right)^{-1} \mathbf{P}_{\mathrm{t}}^{\top} \mathbf{W}\right) \mathbf{Y}_{\mathrm{s}} \mathbf{x}=\mathbf{0}$,

$$
\mathbf{W} \mathbf{Y}_{\mathrm{s}} \mathbf{x}=\mathbf{W P}_{\mathrm{t}} \mathbf{y}
$$

with $\mathbf{y}=\left(\mathbf{P}_{\mathrm{t}}^{\top} \mathbf{W} \mathbf{P}_{\mathrm{t}}\right)^{-1} \mathbf{P}_{\mathrm{t}}^{\top} \mathbf{W} \mathbf{Y}_{\mathrm{s}} \mathbf{x}$. Using Proposition 6, we make the splitting

$$
\mathbf{Y}_{\mathrm{S}} \mathbf{x}=\widetilde{\mathbf{S}}^{\top} \mathbf{x}_{1}+\mathbf{M}_{\mu}^{-1} \mathbf{C}^{\top} \mathbf{x}_{2} \quad \text { and } \quad \mathbf{P}_{\mathrm{t}} \mathbf{y}=\widetilde{\mathbf{S}}^{\top} \mathbf{y}_{1}+\mathbf{M}_{\mu}^{-1} \mathbf{C}^{\top} \mathbf{y}_{2},
$$

and rewrite the equality (4.32) with the splitting as

$$
\mathbf{W} \mathbf{Y}_{\mathrm{s}} \mathbf{x}=\mathbf{C}^{\top} \mathbf{x}_{2}=\mathbf{W P}_{\mathrm{t}} \mathbf{y}=\mathbf{C}^{\top} \mathbf{y}_{2} .
$$


Assumption 17 states that, for $\mathbf{x}, \mathbf{y} \neq 0$,

$$
\mathbf{C M}_{\mu}^{-1} \mathbf{C}^{\top} \mathbf{x}_{2}=\mathbf{C} \mathbf{Y}_{\mathrm{s}} \mathbf{x} \neq \mathbf{C P}_{\mathrm{t}} \mathbf{y}=\mathbf{C M}_{\mu}^{-1} \mathbf{C}^{\top} \mathbf{y}_{2}
$$

and thus $\mathbf{C}^{\top} \mathbf{x}_{2} \neq \mathbf{C}^{\top} \mathbf{y}_{2}$ and $\mathbf{W} \mathbf{Y}_{\mathrm{s}} \mathbf{x} \neq \mathbf{W} \mathbf{P}_{\mathrm{t}} \mathbf{y}$. Therefore, $\mathbf{x}^{\top} \mathbf{L}_{\mu} \mathbf{x}=0$ implies $\mathbf{x}=0$ and thus $\mathbf{L}_{\mu}$ is positive definite. Inverting it in (4.31) yields the expression

$$
\frac{\mathrm{d}}{\mathrm{d} t} \mathbf{i}_{\mu}=\mathbf{L}_{\mu}^{-1} \mathbf{v}_{\mu}+\mathbf{L}_{\mu}^{-1} \mathbf{Y}_{\mathrm{s}}^{\top} \mathbf{W} \mathbf{P}_{\mathrm{t}}\left(\mathbf{P}_{\mathrm{t}}^{\top} \mathbf{W} \mathbf{P}_{\mathrm{t}}\right)^{-1} \mathbf{K}_{\rho} \mathbf{t}_{\mathrm{red}}
$$

The combination of (4.28), (4.30) and (4.33) yields an ODE for the internal variables $\Psi$ and $\mathbf{t}_{\mathrm{red}}$.

Furthermore, equation (4.33) with the positive definite $\mathbf{L}_{\mu}$ is like (4.20), which concludes the proof.

Again, like in the case of its dual formulation, the magnetoquasistatic $\vec{T}-\Omega$ DAE coupled to a circuit has the same index behaviour as inductances. The same corollary follows for the particular circuit that couples the DAE to either a voltage or a current source.

Corollary 2 (Excitation index of the $\vec{T}-\Omega$ formulation [55]). Let Assumptions 16 and 17 hold, then the semidiscrete, gauged system of equations of the $\vec{T}-\Omega$ formulation together with the circuit coupling equation (4.27) has differential index

- 1 , if the voltage $\mathbf{v}_{\mu}$ is given as a function of time.

- 2 , if the current $\mathbf{i}_{\mu}$ is given as a function of time.

Thus in both magnetoquasistatic formulations, a voltage excitation is structurally preferable, as the resulting DAE has a lower index and as a consequence conveys less numerical and analytical difficulties.

\section{Field-dependent materials}

For the analysis of both the $\vec{A}^{*}$ (4.22) and the $\vec{T}-\Omega$ (4.27) formulations we considered materials that had no field dependencies and thus were only space dependent. However, the analysis is easily expandable for fielddependent material matrices. In that case, the reluctivity matrix $\mathbf{M}_{\nu}(\mathbf{b})$ and the permeability matrix $\mathbf{M}_{\mu}(\mathbf{h})$ depend on the semidiscrete magnetic flux density $\mathbf{b}=\mathbf{C a}$ and magnetic field strength $\mathbf{h}=\mathbf{C t}+\widetilde{\mathbf{S}}^{\top} \boldsymbol{\Psi}+\mathbf{Y}_{\mathrm{s}} \mathbf{i}_{\mu}$, respectively. This has to be taken into account whenever time derivatives are taken and the chain rule has to be applied. For example, Faraday's law (4.27a) as well as the circuit coupling equation (4.27c) in the $\vec{T}-\Omega$ DAE are

$$
\begin{aligned}
& \mathbf{K}_{\rho} \mathbf{t}_{\text {red }}+\frac{\mathrm{d}}{\mathrm{d} t}\left(\mathbf{M}_{\mu}(\mathbf{h}) \mathbf{h}\right)=0, \quad \text { and } \\
& \frac{\mathrm{d}}{\mathrm{d} t}\left(\mathbf{Y}_{\mathrm{s}}^{\top} \mathbf{M}_{\mu}(\mathbf{h}) \mathbf{h}\right)-\mathbf{v}_{\mu}=0 .
\end{aligned}
$$

Applying the chain rule

$$
\frac{\mathrm{d}}{\mathrm{d} t}\left(\mathbf{M}_{\mu}(\mathbf{h}) \mathbf{h}\right)=\frac{\partial}{\partial \mathbf{h}}\left(\mathbf{M}_{\mu}(\mathbf{h}) \mathbf{h}\right) \frac{\mathrm{d}}{\mathrm{d} t} \mathbf{h}
$$


yields

$$
\begin{aligned}
\mathbf{K}_{\rho} \mathbf{t}_{\text {red }}+\mathbf{M}_{\mu, \mathrm{d}}(\mathbf{h}) \frac{\mathrm{d}}{\mathrm{d} t} \mathbf{h} & =0, \quad \text { and } \\
\mathbf{Y}_{\mathrm{s}}^{\top} \mathbf{M}_{\mu, \mathrm{d}}(\mathbf{h}) \frac{\mathrm{d}}{\mathrm{d} t} \mathbf{h}-\mathbf{v}_{\mu}=0 &
\end{aligned}
$$

where $\mathbf{M}_{\mu, \mathrm{d}}(\mathbf{h})$ is the differential permeability matrix, which is built analogous to the classic permeability matrix by considering the differential permeability $\boldsymbol{\mu}_{\mathrm{d}}$ from Section 2.2.1 instead of the chord [67] material $\boldsymbol{\mu}$. A detailed proof of this chain rule differentiation step for the reluctivity matrix in the $\vec{A}^{*}$ formulation can be found in [113, Appendix A.3] and [70, Chapter 3]. The permeability matrix follows analogously. Therefore, every instance of the chord material matrices $\mathbf{M}_{\mu}$ and $\mathbf{M}_{\nu}$ is replaced by the differential material matrices $\mathbf{M}_{\mu, \mathrm{d}}$ and $\mathbf{M}_{\nu, \mathrm{d}}$, whenever a time derivative is taken for field-dependent materials. As the differential material tensors are positive definite, whenever Assumption 4 on the $\mathrm{BH}$ curve is given (see Proposition 1), the differential material matrices are also positive definite and the inductance-like proofs for Propositions 4 and 7 can be immediately transferred to the case of field-dependent materials.

Corollary 3. Provided Assumptions 15, 16 and 17 hold, then the semidiscrete, gauged $\vec{A}^{*}$ and $\vec{T}-\Omega$ DAE with circuit coupling equation and field-dependent materials (4.22) and (4.27) are inductance-like elements.

Proposition 8 (Linear index 2 components). The semidiscrete, gauged $\vec{T}-\Omega$ (4.27) and $\vec{A}^{*}$ (4.22) systems of equations fulfilling Assumptions 15, 16 and 17 with field-dependent materials and coupled to circuits described with MNA lead to DAEs with linear index 2 components.

Proof. The proof follows immediately, as both systems (4.27) and (4.22) are inductance-like elements with the structure described in Proposition 2.

As it is explained in Section 2.5.6, the $\vec{A}^{*}$ and the $\vec{T}-\Omega$ formulations are complementary and live on spaces that are dual to each other in Maxwell's house. In the extension of the diagram of Figure 2.5, it can also be visualised that the voltages and current excitations are also dual to each other. Combining these two properties could lead to the intuition that, whereas for the $\vec{A}^{*}$ formulation a voltage excitation is more convenient from the index point of view, for the $\vec{T}-\Omega$ system a current excitation could be better. This would correspond to exciting the problem with the quantity that is on the same side of the diagram as the potentials of the corresponding formulation. However, the analysis shows that this is not the case, as both systems are inductance-like elements and voltage excitations lead to index 1 whereas imposing the current yields index 2 systems. Therefore, here the index results are rather connected to the physics, that describes inductive and resistive effects, than the formulation.

\subsubsection{Capacitance-like element}

The next approximation of Maxwell's equations we conside is the PDE for electroquasistatics in terms of the electric scalar potential presented in Section 2.5.2. We follow the structure of the analysis in [24] and again, for simplicity of notation, consider field-independent materials.

First, a spatial discretisation is applied to the BVP composed by the PDE for eletroquasistatics with the excitation through inhomogeneous boundary conditions (2.37) and the circuit coupling equation (2.37). For 
the discrete version of the Dirichlet lift and thus to incorporate the inhomogeneous boundary conditions, we start by defining the total electric scalar potential

$$
\bar{\phi}=\phi+\mathbf{\Upsilon}_{\varepsilon}
$$

and consider its space discrete counterpart $\overline{\mathbf{\Phi}}$. The spatial discretisation matrices are obtained with only homogeneous boundary conditions on $\Gamma_{\text {dir }, 0}$ and $\Gamma_{\text {neu }, 0}$ and then we define a projector $\overline{\mathbf{P}}_{\mathrm{s}}$ onto the degrees of freedom of $\overline{\mathbf{\Phi}}$ lying on $\Gamma_{\mathrm{s}}$. Its complementary projector $\overline{\mathbf{Q}}_{\mathrm{s}}=I-\overline{\mathbf{P}}_{\mathrm{s}}$ is truncated to obtain a matrix $\mathbf{Q}_{\mathrm{s}}$ with full column rank, by deleting the linear dependent columns. Now we can split the semidiscrete total electric scalar potential as

$$
\overline{\mathbf{\Phi}}=\mathbf{Q}_{\mathrm{s}} \mathbf{\Phi}+\mathbf{Y}_{\mathrm{s}} \mathbf{v}_{\varepsilon},
$$

where $\mathbf{Y}_{\mathrm{S}}$ is the spatial discretisation of the Dirichlet lift function tensor $\mathbf{\Upsilon}$ and $\mathbf{Q}_{\mathrm{S}} \boldsymbol{\Phi}$ represent the remaining degrees of freedom.

Proposition 9 (Full column rank excitation). The spatial discretisation matrix of the Dirichlet lift function $\mathbf{Y}_{\mathrm{s}}$ has full column rank.

Proof. This proposition follows from the definition of the continuous Dirichlet lift function (Definition 5), whose $k$-th column is only nonzero in $\Gamma_{\mathrm{s}}^{(k)}$ and zero everywhere else of $\Gamma$. As the different ports $\Gamma_{\mathrm{s}}^{(k)}$ are disjoint between each other by construction, $\left(\mathbf{Y}_{\mathrm{S}}\right)_{i, j} \neq 0$, for $\left(\mathbf{Y}_{\mathrm{S}}\right)_{i, j}$ being in $\Gamma_{\mathrm{S}}^{(j)}$, whereas $\left(\mathbf{Y}_{\mathrm{s}}\right)_{i, k}=0$, for $k \neq j$. Therefore, the columns are linearly independent and the matrix has full column rank.

The semidiscrete eletroquasistatic field DAE [24], [58] can be written as

$$
\begin{aligned}
& \mathbf{Q}_{\mathrm{s}}^{\top} \mathbf{L}_{\sigma} \mathbf{Q}_{\mathrm{s}} \boldsymbol{\Phi}+\mathbf{Q}_{\mathrm{s}}^{\top} \mathbf{L}_{\varepsilon} \mathbf{Q}_{\mathrm{s}} \frac{\mathrm{d}}{\mathrm{d} t} \boldsymbol{\Phi}+\mathbf{Q}_{\mathrm{s}}^{\top} \mathbf{L}_{\sigma} \mathbf{Y}_{\mathrm{s}} \mathbf{v}_{\varepsilon}+\mathbf{Q}_{\mathrm{s}}^{\top} \mathbf{L}_{\varepsilon} \mathbf{Y}_{\mathrm{s}} \frac{\mathrm{d}}{\mathrm{d} t} \mathbf{v}_{\varepsilon}=0, \\
& \mathbf{Y}_{\mathrm{s}}^{\top} \mathbf{L}_{\sigma} \mathbf{Q}_{\mathrm{s}} \boldsymbol{\Phi}+\mathbf{Y}_{\mathrm{s}}^{\top} \mathbf{L}_{\varepsilon} \mathbf{Q}_{\mathrm{s}} \frac{\mathrm{d}}{\mathrm{d} t} \boldsymbol{\Phi}+\mathbf{Y}_{\mathrm{s}}^{\top} \mathbf{L}_{\sigma} \mathbf{Y}_{\mathrm{s}} \mathbf{v}_{\varepsilon}+\mathbf{Y}_{\mathrm{s}}^{\top} \mathbf{L}_{\varepsilon} \mathbf{Y}_{\mathrm{s}} \frac{\mathrm{d}}{\mathrm{d} t} \mathbf{v}_{\varepsilon}=\mathbf{i}_{\varepsilon},
\end{aligned}
$$

where $\mathbf{L}_{\sigma}=\widetilde{\mathbf{S}} \mathbf{M}_{\sigma} \widetilde{\mathbf{S}}^{\top}$ and $\mathbf{L}_{\varepsilon}=\widetilde{\mathbf{S}} \mathbf{M}_{\varepsilon} \widetilde{\mathbf{S}}^{\top}$ are two generalised Laplace matrices.

Proposition 10 (Discrete lift function [24]). Given the truncated projector matrix $\mathbf{Q}_{\mathrm{s}}$ and the spatial discretisation of the Dirichlet lift function $\mathbf{Y}_{\mathrm{S}}$,

$$
\mathbf{Q}_{\mathrm{s}} \mathbf{x}_{1} \neq \mathbf{Y}_{\mathrm{s}} \mathbf{x}_{2}, \text { for } \mathbf{x}_{1}, \mathbf{x}_{2} \neq 0
$$

Proof. This property follows directly from the definition of both matrices. The discrete excitation matrix $\left(\mathbf{Y}_{\mathrm{s}}\right)_{i, j} \neq 0$ for $\left(\mathbf{Y}_{\mathrm{s}}\right)_{i, j}$ in $\Gamma_{\mathrm{s}}^{(j)}$. However, the image of $\mathbf{Q}_{\mathrm{s}}$ is the complementary of the image of $\overline{\mathbf{P}}_{\mathrm{S}}$ and therefore projects onto the complementary of $\Gamma_{\mathrm{s}}$. Due to Proposition 9, $\mathbf{Y}_{\mathrm{s}}$ has full column rank and by construction also $\mathbf{Q}_{\mathrm{s}}$. Therefore both have a trivial kernel and, as their images are complementary, the proposition holds.

Proposition 11 (Electroquasistatic capacitance-like element [24]). The semidiscrete system of differential equations arising from an electroquasistatic setting with circuit coupling equation (4.34) is a capacitance-like element. 
Proof. We first try to write an ODE for the internal degrees of freedom $\mathbf{x}_{\varepsilon}=\boldsymbol{\Phi}$ in terms of $\boldsymbol{\Phi}, \mathbf{v}_{\varepsilon}$ and $\mathbf{i}_{\varepsilon}$.

Due to Assumption 11 and Lemma 2, $\mathbf{L}_{\varepsilon}$ is positive definite and, using the full column rank of $\mathbf{Q}_{\mathrm{s}}$, we extract from (4.34a) without the need of any time differentiation

$$
\begin{aligned}
\frac{\mathrm{d}}{\mathrm{d} t} \boldsymbol{\Phi}= & -\left(\mathbf{Q}_{\mathrm{s}}^{\top} \mathbf{L}_{\varepsilon} \mathbf{Q}_{\mathrm{s}}\right)^{-1} \mathbf{Q}_{\mathrm{s}}^{\top} \mathbf{L}_{\sigma} \mathbf{Q}_{\mathrm{s}} \boldsymbol{\Phi} \\
& -\left(\mathbf{Q}_{\mathrm{s}}^{\top} \mathbf{L}_{\varepsilon} \mathbf{Q}_{\mathrm{s}}\right)^{-1}\left(\mathbf{Q}_{\mathrm{s}}^{\top} \mathbf{L}_{\varepsilon} \mathbf{Y}_{\mathrm{s}} \frac{\mathrm{d}}{\mathrm{d} t} \mathbf{v}_{\varepsilon}+\mathbf{Q}_{\mathrm{s}}^{\top} \mathbf{L}_{\sigma} \mathbf{Y}_{\mathrm{s}} \mathbf{V}_{\varepsilon}\right) .
\end{aligned}
$$

The expression for $\frac{\mathrm{d}}{\mathrm{d} t} \boldsymbol{\Phi}$ can now be inserted into (4.34b) to obtain

$$
\begin{aligned}
\mathbf{i}_{\varepsilon}= & \mathbf{Y}_{\mathrm{S}}^{\top}\left(\mathbf{I}-\mathbf{L}_{\varepsilon} \mathbf{Q}_{\mathrm{s}}\left(\mathbf{Q}_{\mathrm{s}}^{\top} \mathbf{L}_{\varepsilon} \mathbf{Q}_{\mathrm{s}}\right)^{-1} \mathbf{Q}_{\mathrm{s}}^{\top}\right) \mathbf{L}_{\sigma} \mathbf{Q}_{\mathrm{s}} \mathbf{\Phi} \\
& +\mathbf{Y}_{\mathrm{s}}^{\top}\left(\mathbf{I}-\mathbf{L}_{\varepsilon} \mathbf{Q}_{\mathrm{s}}\left(\mathbf{Q}_{\mathrm{s}}^{\top} \mathbf{L}_{\varepsilon} \mathbf{Q}_{\mathrm{s}}\right)^{-1} \mathbf{Q}_{\mathrm{S}}^{\top}\right) \mathbf{L}_{\sigma} \mathbf{Y}_{\mathrm{s}} \mathbf{v}_{\varepsilon} \\
& +\mathbf{Y}_{\mathrm{S}}^{\top}\left(\mathbf{L}_{\varepsilon}-\mathbf{L}_{\varepsilon} \mathbf{Q}_{\mathrm{s}}\left(\mathbf{Q}_{\mathrm{S}}^{\top} \mathbf{L}_{\varepsilon} \mathbf{Q}_{\mathrm{S}}\right)^{-1} \mathbf{Q}_{\mathrm{S}}^{\top} \mathbf{L}_{\varepsilon}\right) \mathbf{Y}_{\mathrm{s}} \frac{\mathrm{d}}{\mathrm{d} t} \mathbf{v}_{\varepsilon}
\end{aligned}
$$

Let us verify that $\mathbf{C}_{\varepsilon}=\mathbf{Y}_{\mathrm{S}}^{\top}\left(\mathbf{L}_{\varepsilon}-\mathbf{L}_{\varepsilon} \mathbf{Q}_{\mathrm{s}}\left(\mathbf{Q}_{\mathrm{S}}^{\top} \mathbf{L}_{\varepsilon} \mathbf{Q}_{\mathrm{S}}\right)^{-1} \mathbf{Q}_{\mathrm{S}}^{\top} \mathbf{L}_{\varepsilon}\right) \mathbf{Y}_{\mathrm{S}}$ is positive definite. As $\mathbf{L}_{\varepsilon}$ is symmetric positive definite, its square root exists and is also symmetric positive definite and we rewrite

$$
\mathbf{C}_{\varepsilon}=\mathbf{Y}_{\mathrm{S}}^{\top} \mathbf{L}_{\varepsilon}^{\frac{1}{2}}\left(\mathbf{I}-\mathbf{L}_{\varepsilon}^{\frac{1}{2}} \mathbf{Q}_{\mathrm{S}}\left(\mathbf{Q}_{\mathrm{s}}^{\top} \mathbf{L}_{\varepsilon} \mathbf{Q}_{\mathrm{S}}\right)^{-1} \mathbf{Q}_{\mathrm{s}}^{\top} \mathbf{L}_{\varepsilon}^{\frac{1}{2}}\right) \mathbf{L}_{\varepsilon}^{\frac{1}{2}} \mathbf{Y}_{\mathrm{S}}
$$

where $\left(\mathbf{I}-\mathbf{L}_{\varepsilon}^{\frac{1}{2}} \mathbf{Q}_{\mathrm{S}}\left(\mathbf{Q}_{\mathrm{s}}^{\top} \mathbf{L}_{\varepsilon} \mathbf{Q}_{\mathrm{s}}\right)^{-1} \mathbf{Q}_{\mathrm{s}}^{\top} \mathbf{L}_{\varepsilon}^{\frac{1}{2}}\right)$ is a symmetric projector and thus positive semidefinite. Therefore, $\mathbf{C}_{\varepsilon}$ is positive semidefinite. Let us consider a vector $\mathbf{x}$, such that $\mathbf{x}^{\top} \mathbf{C}_{\varepsilon} \mathbf{x}=0$, then,

$$
\left(\mathbf{I}-\mathbf{L}_{\varepsilon}^{\frac{1}{2}} \mathbf{Q}_{\mathrm{S}}\left(\mathbf{Q}_{\mathrm{S}}^{\top} \mathbf{L}_{\varepsilon} \mathbf{Q}_{\mathrm{S}}\right)^{-1} \mathbf{Q}_{\mathrm{S}}^{\top} \mathbf{L}_{\varepsilon}^{\frac{1}{2}}\right) \mathbf{L}_{\varepsilon}^{\frac{1}{2}} \mathbf{Y}_{\mathrm{S}} \mathbf{X}=0
$$

This implies

$$
\mathbf{L}_{\varepsilon}^{\frac{1}{2}} \mathbf{Y}_{\mathrm{S}} \mathbf{x}=\mathbf{L}_{\varepsilon}^{\frac{1}{2}} \mathbf{Q}_{\mathrm{S}}\left(\mathbf{Q}_{\mathrm{S}}^{\top} \mathbf{L}_{\varepsilon} \mathbf{Q}_{\mathrm{S}}\right)^{-1} \mathbf{Q}_{\mathrm{S}}^{\top} \mathbf{L}_{\varepsilon} \mathbf{Y}_{\mathrm{S}} \mathbf{X}
$$

whose multiplication by $\mathbf{L}_{\varepsilon}^{-\frac{1}{2}}$ yields the equality $\mathbf{Y}_{\mathrm{s}} \mathbf{x}=\mathbf{Q}_{\mathrm{s}} \mathbf{y}$, where $\mathbf{y}=\left(\mathbf{Q}_{\mathrm{S}}^{\top} \mathbf{L}_{\varepsilon} \mathbf{Q}_{\mathrm{S}}\right)^{-1} \mathbf{Q}_{\mathrm{S}}^{\top} \mathbf{L}_{\varepsilon} \mathbf{Y}_{\mathrm{s}} \mathbf{x}$. Using Proposition 10 and 9 this implies $\mathbf{x}=0$. Thus $\mathbf{C}_{\varepsilon}$ has full rank and is therefore positive definite.

Inverting $\mathbf{C}_{\varepsilon}$ in (4.36) and inserting into (4.35) yields an ODE for the internal variable $\frac{\mathrm{d}}{\mathrm{d} t} \boldsymbol{\Phi}$ as a function of $\Phi, \mathbf{v}_{\varepsilon}$ and $\mathbf{i}_{\varepsilon}$, without the need of any time differentiation of the original system.

In addition, the inversion of $\mathbf{C}_{\varepsilon}$ in (4.36) yields an equation like (4.8) that fulfills the required properties of capacitance-like elements in Definition 20.

Considering the special circuit case of coupling the electroquasistatic differential equation to a voltage or current source yields the following corollary.

Corollary 4 (Excitation index of the electroquasistatic formulation). The semidiscrete system of differential equations of electroquasistatics together with the circuit coupling equation (4.34) has differential index

- 1 , if the current $\mathbf{i}_{\varepsilon}$ is given as a function of time.

- 2 , if the voltage $\mathbf{v}_{\varepsilon}$ is given as a function of time.

Therefore, in contrast to the inductance-like elements, here a current excitation is preferred, as this yields a lower index DAE than prescribing the voltage. 


\section{Field-dependent materials}

Analogous to the examples presented for the inductance-like elements, in the analysis we only considered linear material relations, that is, materials that only depended on space. However, again, the extension of our results to field-dependent materials can be easily obtained. In this case the conductivity $\sigma$ and the permittivity $\varepsilon$ depend on the semidiscrete electric field strength $\mathbf{e}=\widetilde{\mathbf{S}}^{\top} \mathbf{Q}_{\mathbf{S}} \boldsymbol{\Phi}+\widetilde{\mathbf{S}}^{\top} \mathbf{Y}_{\mathbf{S}} \mathbf{v}_{\varepsilon}$. The field dependencies have to be taken into account on the time derivatives of the electric flux density in Ampère's law and the circuit coupling equation

$$
\begin{aligned}
\mathbf{Q}_{\mathrm{s}}^{\top} \widetilde{\mathbf{S}}\left(\mathbf{M}_{\sigma}(\mathbf{e}) \mathbf{e}\right)+\mathbf{Q}_{\mathrm{s}}^{\top} \widetilde{\mathbf{S}} \frac{\mathrm{d}}{\mathrm{d} t}\left(\mathbf{M}_{\varepsilon}(\mathbf{e}) \mathbf{e}\right)=0, \quad \text { and } \\
\mathbf{Y}_{\mathrm{S}}^{\top}\left(\mathbf{M}_{\sigma}(\mathbf{e}) \mathbf{e}\right)+\mathbf{Y}_{\mathrm{s}}^{\top} \frac{\mathrm{d}}{\mathrm{d} t}\left(\mathbf{M}_{\varepsilon}(\mathbf{e}) \mathbf{e}\right)-\mathbf{i}_{\varepsilon}=0 .
\end{aligned}
$$

For all time derivatives we apply the chain rule and obtain the final system

$$
\begin{aligned}
& \mathbf{Q}_{\mathrm{s}}^{\top} \mathbf{L}_{\sigma} \mathbf{Q}_{\mathrm{s}} \boldsymbol{\Phi}+\mathbf{Q}_{\mathrm{s}}^{\top} \mathbf{L}_{\varepsilon, \mathrm{d}} \mathbf{Q}_{\mathrm{s}} \frac{\mathrm{d}}{\mathrm{d} t} \boldsymbol{\Phi}+\mathbf{Q}_{\mathrm{s}}^{\top} \mathbf{L}_{\sigma} \mathbf{Y}_{\mathrm{s}} \mathbf{v}_{\varepsilon}+\mathbf{Q}_{\mathrm{s}}^{\top} \mathbf{L}_{\varepsilon, \mathrm{d}} \mathbf{Y}_{\mathrm{s}} \frac{\mathrm{d}}{\mathrm{d} t} \mathbf{v}_{\varepsilon}=0 \\
& \mathbf{Y}_{\mathrm{s}}^{\top} \mathbf{L}_{\sigma} \mathbf{Q}_{\mathrm{s}} \boldsymbol{\Phi}+\mathbf{Y}_{\mathrm{s}}^{\top} \mathbf{L}_{\varepsilon, \mathrm{d}} \mathbf{Q}_{\mathrm{s}} \frac{\mathrm{d}}{\mathrm{d} t} \boldsymbol{\Phi}+\mathbf{Y}_{\mathrm{s}}^{\top} \mathbf{L}_{\sigma} \mathbf{Y}_{\mathrm{s}} \mathbf{v}_{\varepsilon}+\mathbf{Y}_{\mathrm{s}}^{\top} \mathbf{L}_{\varepsilon, \mathrm{d}} \mathbf{Y}_{\mathrm{s}} \frac{\mathrm{d}}{\mathrm{d} t} \mathbf{v}_{\varepsilon}=\mathbf{i}_{\varepsilon},
\end{aligned}
$$

with $\mathbf{L}_{\varepsilon, \mathrm{d}}(\mathbf{e}):=\widetilde{\mathbf{S}} \mathbf{M}_{\varepsilon, \mathrm{d}}(\mathbf{e}) \widetilde{\mathbf{S}}^{\top}$, where $\mathbf{M}_{\varepsilon, \mathrm{d}}$ is the differential permittivity matrix. Again the chord material matrices are to be replaced by the differential material matrices whenever a time derivative is taken. As due to Assumption 5 the differential permittivity is positive definite, all the previous proofs hold immediately also for field-depentent materials.

Corollary 5. The semidiscrete electroquasistatic differential equation with circuit coupling equation and fielddependent materials (4.34) is a capacitance-like element.

Proposition 12 (Electroquasistatic linear index 2 components). The semidiscrete electroquasistatic differential equation with field-dependent materials and coupled to circuits described with MNA leads to a DAE with linear index 2 components.

Proof. This follows immediately as the index 2 components only depend on the voltage sources and the inductance-like elements (see Proposition 2).

Remark 25. Please note that, as it has been stated before, for capacitance-like elements coupled to circuits through the currents $\mathbf{i}_{\varepsilon}$ instead of the charges $\mathbf{q}_{\varepsilon}$, also $\mathbf{i}_{\varepsilon}$ can be an index 2 component on loops of only capacitances. However, as $\mathbf{i}_{\varepsilon}$ also appears linearly on the electroquasistatic differential equation with circuit coupling equation (4.34), the index 2 components appear linearly in the original DAE even for the case of coupling through the currents.

\subsubsection{Resistance-like element}

For the next refined model we consider the curl-curl equation for the simulation of eddy current effects in magnets with superconducting coils presented in Section 2.7. Again, for simplicity, we consider field-independent materials at the beginning. The analysis and results of this section were first presented in [24]. 
We consider the PDE of the inter-filament coupling currents (2.40), whose excitation is performed through the source current density coupled to the circuit with the winding function (2.30) and the circuit coupling equation (2.29). Applying a spatial discretisation, the system of DAEs

$$
\begin{aligned}
\mathbf{C}^{\top} \mathbf{M}_{\nu, \tau_{\mathrm{eq}}} \mathbf{C} \frac{\mathrm{d}}{\mathrm{d} t} \mathbf{a}+\mathbf{C}^{\top} \mathbf{M}_{\nu} \mathbf{C a}=\mathbf{X}_{\mathrm{s}} \mathbf{i}_{\rho} \\
\frac{\mathrm{d}}{\mathrm{d} t} \mathbf{X}_{\mathrm{s}}^{\top} \mathbf{a}-\mathbf{v}_{\rho}=0
\end{aligned}
$$

is obtained, where $\mathbf{M}_{\nu, \tau_{\mathrm{eq}}}$ is the material matrix for the product of the reluctivity and the cable time constant $\nu \tau_{\text {eq }}$ of (2.39). For the analysis we start by defining the orthogonal projector $\mathbf{Q}_{\tau}$ onto ker $\mathbf{C}^{\top} \mathbf{M}_{\nu, \tau_{\mathrm{eq}}} \mathbf{C}$ and its complementary as $\mathbf{P}_{\tau}=I-Q_{\tau}$. This allows to state the following assumptions.

Assumption 18 (Excitation of homogenisation model [24]). We assume that

(i) the discrete magnetic vector potential $\mathbf{a}$ is gauged such that $\mathbf{C}$ has full column rank.

(ii) the circuit excitation is only performed on the coils' region, i.e., $\mathbf{Q}_{\tau}^{\top} \mathbf{X}_{\mathrm{s}}=0$.

The first part of the assumption is required to obtain a uniquely solvable system (4.37). We do not explicitly apply a gauging condition to our system, as we only consider two dimensional magnets cross-sections. For two dimensional settings where the magnetic vector potential only has one perpendicular component that depends on the other two dimensions (e.g. $\vec{A}=A_{z}(x, y) \vec{e}_{z}$ ), the gauging condition is automatically fulfilled.

The second part of the assumption is motivated as the discrete counterpart of Assumption 8. Here, both the eddy currents as well as the circuit coupling is performed on the region of the coils $\Omega_{\mathrm{s}}$.

Proposition 13 (Homogenisation model resistance-like element [24]). Given Assumption 18 holds, then the semidiscrete homogenisation eddy current system for inter-filament coupling currents with circuit coupling equation (4.37) is a resistance-like element.

Proof. Again we start by trying to write an ODE for the internal degrees of freedom $\mathbf{x}_{\rho}=\mathbf{a}$.

We first split the equation (4.37a) with the projectors $\mathbf{Q}_{\tau}^{\top}$ and $\mathbf{P}_{\tau}^{\top}$ into

$$
\mathbf{C}^{\top} \mathbf{M}_{\nu, \tau_{\mathrm{eq}}} \mathbf{C} \frac{\mathrm{d}}{\mathrm{d} t} \mathbf{a}+\mathbf{P}_{\tau}^{\top} \mathbf{C}^{\top} \mathbf{M}_{\nu} \mathbf{C a}=\mathbf{P}_{\tau}^{\top} \mathbf{X}_{\mathrm{s}} \mathbf{i}_{\rho}
$$

and

$$
\mathbf{Q}_{\tau}^{\top} \mathbf{C}^{\top} \mathbf{M}_{\nu} \mathbf{C a}=\mathbf{Q}_{\tau}^{\top} \mathbf{X}_{\mathrm{s}} \mathbf{i}_{\rho}
$$

Without the need of any time differentiation and due to the definition of $\mathbf{P}_{\tau}^{\top}$, we can extract from (4.38) an ODE for $\mathbf{P}_{\tau} \frac{\mathrm{d}}{\mathrm{d} t} \mathbf{a}$

$$
\mathbf{P}_{\tau} \frac{\mathrm{d}}{\mathrm{d} t} \mathbf{a}=\left(\mathbf{C}^{\top} \mathbf{M}_{\nu, \tau_{\mathrm{eq}}} \mathbf{C}+\mathbf{Q}_{\tau}^{\top} \mathbf{Q}_{\tau}\right)^{-1}\left(\mathbf{P}_{\tau}^{\top} \mathbf{X}_{\mathrm{s}} \mathbf{i}_{\rho}-\mathbf{P}_{\tau}^{\top} \mathbf{C}^{\top} \mathbf{M}_{\nu} \mathbf{C a}\right) .
$$

One time differentiation of (4.39) and exploiting Assumption 18 as well as the positive definitenes of $\mathbf{M}_{\nu}$ and the definition of the projector matrices yields

$$
\mathbf{Q}_{\tau} \frac{\mathrm{d}}{\mathrm{d} t} \mathbf{a}=-\left(\mathbf{Q}_{\tau}^{\top} \mathbf{C}^{\top} \mathbf{M}_{\nu} \mathbf{C} \mathbf{Q}_{\tau}+\mathbf{P}_{\tau}^{\top} \mathbf{P}_{\tau}\right)^{-1} \mathbf{Q}_{\tau}^{\top} \mathbf{C}^{\top} \mathbf{M}_{\nu} \mathbf{C} \mathbf{P}_{\tau} \frac{\mathrm{d}}{\mathrm{d} t} \mathbf{a} .
$$


An ODE for $\mathbf{Q}_{\tau} \frac{\mathrm{d}}{\mathrm{d} t} \mathbf{a}$ is obtained by inserting (4.40) into (4.41), which yields an ODE for the internal degrees of freedom $\mathbf{x}_{\rho}=\left(\mathbf{P}_{\tau}+\mathbf{Q}_{\tau}\right) \mathbf{a}$ with one time differentiation of the original DAE.

Using again Assumption 18 and inserting the ODE (4.40) into the circuit coupling equation yields

$$
\begin{aligned}
\mathbf{v}_{\rho} & =\mathbf{X}_{\mathrm{s}}^{\top} \frac{\mathrm{d}}{\mathrm{d} t}\left(\mathbf{P}_{\tau} \mathbf{a}+\mathbf{Q}_{\tau} \mathbf{a}\right)=\mathbf{X}_{\mathrm{s}}^{\top} \frac{\mathrm{d}}{\mathrm{d} t} \mathbf{P}_{\tau} \mathbf{a} \\
& =\mathbf{X}_{\mathrm{s}}^{\top} \mathbf{P}_{\tau}\left(\mathbf{C}^{\top} \mathbf{M}_{\nu, \tau_{\mathrm{eq}}} \mathbf{C}+\mathbf{Q}_{\tau}^{\top} \mathbf{Q}_{\tau}\right)^{-1}\left(\mathbf{P}_{\tau}^{\top} \mathbf{X} \mathbf{i}_{\rho}-\mathbf{P}_{\tau}^{\top} \mathbf{C}^{\top} \mathbf{M}_{\nu} \mathbf{C a}\right) .
\end{aligned}
$$

Please note that no time differentiation of the original system was required to obtain this expression. Differentiating this once and inserting again (4.40) gives the expression

$$
\frac{\mathrm{d}}{\mathrm{d} t} \mathbf{v}_{\rho}=\mathbf{G}_{\rho}^{-1} \frac{\mathrm{d}}{\mathrm{d} t} \mathbf{i}_{\rho}+\mathbf{f}_{\tau}\left(\mathbf{a}, \mathbf{i}_{\rho}\right)
$$

with a positive definite $\mathbf{G}_{\rho}^{-1}:=\mathbf{X}_{\mathrm{s}}^{\top} \mathbf{P}_{\tau}\left(\mathbf{C}^{\top} \mathbf{M}_{\nu, \tau_{\mathrm{eq}}} \mathbf{C}+\mathbf{Q}_{\tau}^{\top} \mathbf{Q}_{\tau}\right)^{-1} \mathbf{P}_{\tau}^{\top} \mathbf{X}_{\mathrm{s}}$. Its positive definiteness follows immediately from the positive semidefiniteness of $\mathbf{M}_{\nu, \tau_{\text {eq }}}$, the definition of the projector matrices and the full column rank of $\mathbf{X}_{\mathrm{s}}$ and Assumption 18. Thus we have obtained an expression of the type (4.12) fulfilling the properties of the resistance-like elements in Definition 21.

Corollary 6 (Excitation index of the homogenisation model). Let Assumption 18 holds, then the semidiscrete homogenisation eddy current system for inter-filament coupling currents with circuit coupling equation (4.37) has differential index

- 1 , if the current $\mathbf{i}_{\rho}$ is given as a function of time.

- 1 , if the voltage $\mathbf{v}_{\rho}$ is given as a function of time.

Thus, in this case, both excitations yield an index 1 system and no preference is given in terms of the index of the DAE.

\section{Field-dependent materials}

Again for the first analysis we considered constant material matrices $\mathbf{M}_{\nu}$ and $\mathbf{M}_{\nu, \tau_{\mathrm{eq}}}$, however, specially in the quench simulation of superconducting accelerator magnets, nonlinearities occur in both material relations, as both strongly depend on the magnetic flux density $\mathbf{b}=\mathbf{C a}$.

Due to the construction of the homogenisation term in (2.39), this nonlinearity only affects the time differentiations performed during the proof of Proposition 13. Therefore, to obtain the ODE term of $\mathbf{Q}_{\tau} \frac{\mathrm{d}}{\mathrm{d} t} \mathbf{a}$ in (4.41), the differential reluctivity matrix $\mathbf{M}_{\nu, \mathrm{d}}$ is obtained, which is also positive definite and thus that part of the proof still holds. The second time differentiation

$$
\frac{\mathrm{d}}{\mathrm{d} t} \mathbf{v}_{\rho}=\frac{\mathrm{d}}{\mathrm{d} t}\left(\mathbf{G}_{\rho}(\mathbf{b})^{-1} \mathbf{i}_{\rho}\right)-\frac{\mathrm{d}}{\mathrm{d} t}\left(\mathbf{X}_{\mathrm{s}}^{\top} \mathbf{P}_{\tau}\left(\mathbf{C}^{\top} \mathbf{M}_{\nu, \tau_{\mathrm{eq}}}(\mathbf{b}) \mathbf{C}+\mathbf{Q}_{\tau}^{\top} \mathbf{Q}_{\tau}\right)^{-1} \mathbf{P}_{\tau}^{\top} \mathbf{C}^{\top} \mathbf{M}_{\nu}(\mathbf{b}) \mathbf{C a}\right)
$$

is taken to obtain the resistance-like element's expression (4.42). Here, the product rule is applied on the expression $\mathbf{G}_{\rho}(\mathbf{b})^{-1} \mathbf{i}_{\rho}$, which, after inserting the ODE expression for $\frac{\mathrm{d}}{\mathrm{d} t} \mathbf{a}$ yields an equation of the same structure like (4.42) and the same definition for $\mathbf{G}_{\rho}(\mathbf{b})^{-1}$, however with a different $\mathbf{f}_{\tau}\left(\mathbf{a}, \mathbf{i}_{\rho}\right)$.

This last eddy current homogenisation model, in contrast to the classical $\vec{A}^{*}$ or $\vec{T}-\Omega$ formulations, is classified as a resistance-like and not an inductance-like element. However, this does not contradict the physics 
described by the equation, as a magnetoquasistatic approximation of Maxwell's equations both describes resistive as well as inductive effects. The eddy current effects described by the homogenisation model are present in the same coils where the excitation of the field problem takes place. This is different from the eddy current effects described by the classical magnetoquasitatic PDEs of the inductance-like examples, where the eddy current effects appear only on the conducting region, which is disjoint from the domain of the excitation coils.

For an intuitive visualisation of how this can happen, we present two different circuits that yield similar results. Let us consider an inductance $(L)$ and resistance $(R)$ connected in series and in parallel. The first case can be described by the equation

$$
\mathbf{v}_{\mu}=L \frac{\mathrm{d}}{\mathrm{d} t} \mathbf{i}_{\mu}+R \mathbf{i}_{\mu},
$$

which clearly is an inductance-like element. However, the second one is governed by the relation

$$
\frac{\mathrm{d}}{\mathrm{d} t} \mathbf{i}_{\rho}=\frac{1}{L} \mathbf{v}_{\rho}+\frac{1}{R} \frac{\mathrm{d}}{\mathrm{d} t} \mathbf{v}_{\rho},
$$

that is classified as a resistance-like element. Both circuits describe resistive and inductive effects, however, similarly to the different magnetoquasistatic models, they behave and are classified differently.

\subsection{Conclusion}

In this chapter, three generalised element definitions have been presented and a topological index result has been derived for circuits containing these type of elements. It has been shown that important approximations of Maxwell's equations can be described by generalisations of resistances, inductances and capacitances. This classification eases the index analysis of circuits containing refined models, as it is enough to analyse the structure of only the refined model to determine which generalised element it corresponds to in results for the entire coupled system in terms of the topology of the circuit.

The analysis of the refined models has confirmed in the case of the $\vec{A}^{*}$ formulation already known results (see [22]) and has also offered new index results for other approximations and formulations of Maxwell's equations [24], [55]. The classification of the different field models yielded surprising outcomes. First the fact that, even though the $\vec{A}^{*}$ and the $\vec{T}-\Omega$ formulations have dual degrees of freedom and the voltage and current excitations are dual too, voltage prescription has resulted in both cases in a lower index 1 system. Second, the resistance-like classification of the eddy current model used for superconducting coils, where both voltage as well as current excitation has yielded an index 1 DAE.

The following section presents the iterative time domains methods that are used for the simulation of multiphysical systems, especially those arising from field-circuit coupling. 


\section{Iterative Methods in Time Domain}

The simulation of large, coupled, multiphysical problems typically poses considerable challenges. Their multiphysical character frequently involves the coupling of systems of equations with different mathematical properties, time rates and sizes, whose coupled solution has to be numerically approximated together with one method. Furthermore, specially when considering space-discretised finite element (or FIT) models, each subsystems can become very large and computationally costly to solve. One such case is the simulation of the LHC's quench protection system [5], where electric circuits are coupled to several eddy current problems and heat equations for each magnet. To simulate such types of systems, special numerical methods shall be presented, that may ease as well as speed up the computation.

In this chapter two time domain simulation algorithms are presented that can be applied to field-circuit coupled systems. Co-simulation techniques (in particular the waveform relaxation (WR) method [25]) are used [5], [32], [137] to exploit the different mathematical properties of the systems. Also, to further speed up the computation time, the Parareal algorithm [42] is presented and adapted to the case of DAEs. It is a parallel-intime method that is able to parallelise the simulation of initial value problems.

The content and structure of this chapter is based on [56], [57] and it is structured as follows: Section 5.1 deals with the waveform relaxation algorithm. At the beginning, the algorithm itself is presented and some already known theoretical results are recalled. WR is an iterative method whose convergence can be sped up by means of optimised Schwarz methods [37], [138]. Here they are applied to field-circuit coupled systems arising from eddy current models for superconducting coils. This yields an optimised waveform relaxation algorithm that can be efficiently applied to the simulation of quenches in accelerator magnets. Section 5.2 deals with Parareal. Again the classic algorithm and known theoretical results are explained at first as well as a special version of the algorithm called micro-macro Parareal [139]. In the end, difficulties the Parareal algorithm may have when used for differential algebraic equations are explained and a modification of the algorithm for its usage on index 2 DAEs is introduced. In the end, a specific structured DAE is studied in terms of Parareal and the implicit Euler method. The last section combines waveform relaxation and Parareal into one algorithm with the focus in field-circuit coupled systems. An improved algorithm based on optimised Schwarz methods and micro-macro Parareal is given.

\subsection{Optimised Waveform Relaxation}

The simulation of coupled multiphysical problems can be dealt with in several ways. They can either be solved in a monolithical way, where the different physical equations are coupled together in a potentially large system [140], [141], or they can be co-simulated [31], [32], [142]. These techniques allow to solve the systems separately and exchange information between them to connect their behaviour. Some algorithms, such as the waveform relaxation method [143], perform this information exchange iteratively and 


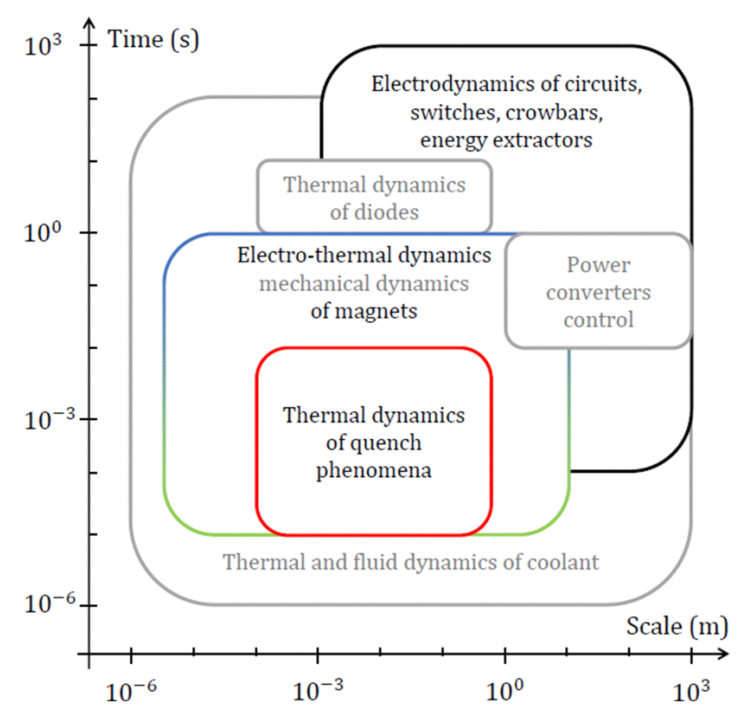

Figure 5.1: Multiscale and multirate behaviour of LHC's quench simulation problem [5]. @ [2018] IEEE.

thus convergence to the coupled (monolithical) solution must be proven for ODEs and even specific structured DAEs [28], [30].

Whereas, at first glance, solving the monolithic system may seem less complicated, this can convey several difficulties and inconveniences. First of all, the different subsystems arising from distinct physical equations may exhibit different time scales (see Figure 5.1) and thus different time step sizes could be potentially used (see e.g. [3]). Furthermore, most existing simulation software is specialised in one type of physics or systems. For example, SPICE solvers are able to simulate electric circuits, whereas other software such as GetDP, CST or COMSOL are specialised in space discretisation of PDEs e.g. arising from electromagnetic field problems. As it has been mentioned before, the systems have different mathematical properties and thus are typically tackled with different time integration techniques and linear solvers. Additionally, some software (especially commercial code) do not allow extracting the system's information that is required to perform a monolithic coupling, such as e.g. the Jacobian of the systems' matrices or even the system matrices itself. Here, only variables in an input-output manner can be exchanged, which makes a monolithic simulation in practice impossible. Exploiting the multirate behaviour, different features of the subsystems and already existing code is possible by means of co-simulation. This allows to solve the systems separately with their own specific software, techniques and time step sizes, and only the co-simulation framework has to be built around them (see e.g. [5]).

Even though the waveform relaxation algorithm can be applied to various types of subsystems such as e.g. [144] for controller-circuit coupling, this work focuses on its usage on field-circuit coupled systems. In these type of problems, a multirate behaviour is expected, which can be exploited by the algorithm (see e.g. [3], [56]). The co-simulation methods are implemented in the STEAM (Simulation of Transient Effects in Accelerator Magnets) platform, which brings a framework that implements the WR method as a hierarchical co-simulation algorithm [5] for the different physics involved in the simulation of quench effects in accelerator magnets.

To speed up the convergence of the WR iteration, optimised Schwarz methods can be used [37], [138]. In [138], these type of methods are used to optimise the convergence of a WR scheme for the one dimensional advection reaction diffusion equation. Other works, such as [38], [39], [145], [146], optimise the information exchange between circuit subsystems or even in field-circuit coupled problems (see [40]). In [144] 


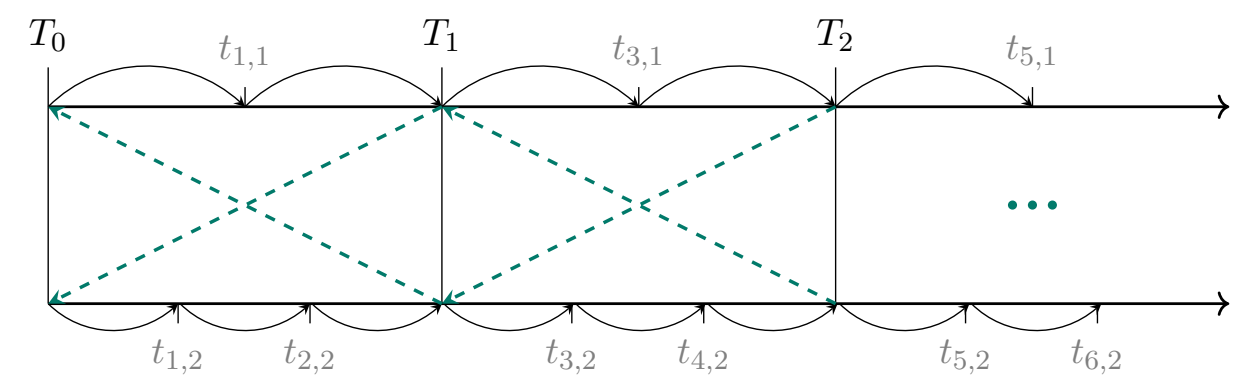

Figure 5.2: Schematic of waveform relaxation algorithm with two subsystems and windowing.

lower and upper bounds for WR iterations are given for the coupling of controller and circuit systems. This section presents the work of [56], where optimised Schwarz waveform relaxation is applied to field-circuit coupled systems arising from the eddy current equation for interfilament coupling currents in superconducting coils.

\subsubsection{Introduction}

In the following we briefly introduce the fundamentals of the waveform relaxation algorithm and its connection to optimised Schwarz methods.

\section{Waveform relaxation}

Waveform relaxation is a co-simulation technique originally introduced for the simulation of large electric networks [25]. Given an initial value problem on time $t \in \mathcal{I}$, the algorithm starts by dividing the original (coupled) monolithic system into $m$ subsystems which are then solved separately. Iteratively, information is exchanged between them so as to converge to the original, monolithic solution.

Often this methodology is not done on the entire time window $\mathcal{I}$, but windowing is performed. Here, $\mathcal{I}$ is divided into $N_{\mathrm{WR}}$ subwindows $\mathcal{I}_{n}^{\mathrm{WR}}=\left[T_{n-1}^{\mathrm{WR}} T_{n}^{\mathrm{WR}}\right), n=1, \ldots, N_{\mathrm{WR}}$ with $T_{0}=t_{0}$ of size $\Delta T_{\mathrm{WR}}$ and the iteration is carried out on only one window. Once convergence up to a certain tolerance is achieved, the scheme is applied on the next window. This is repeated until all subwindows are simulated. For a schematic of the waveform relaxation algorithm with two subsystems and windowing see Figure 5.2.

Remark 26. Note that all windows $\mathcal{I}_{n}, n=1, \ldots, N_{\mathrm{WR}}$ do not necessarily have to be of the same size. One possibility is to dynamically change the window size for convergence speed reasons with some window size control algorithm [147]. This is of particular importance if no or only very few iterations are to be used within the co-simulation algorithm (e.g. weak coupling) and for real-time simulations. For simplicity of notation we will not consider windows of different sizes.

Let us consider the initial value problem with consistent initial condition

$$
\begin{aligned}
\mathbf{F}\left(\frac{\mathrm{d}}{\mathrm{d} t} \mathbf{x}, \mathbf{x}, t\right) & =0 \\
\mathbf{x}\left(t_{0}\right) & =\mathbf{x}_{0},
\end{aligned}
$$




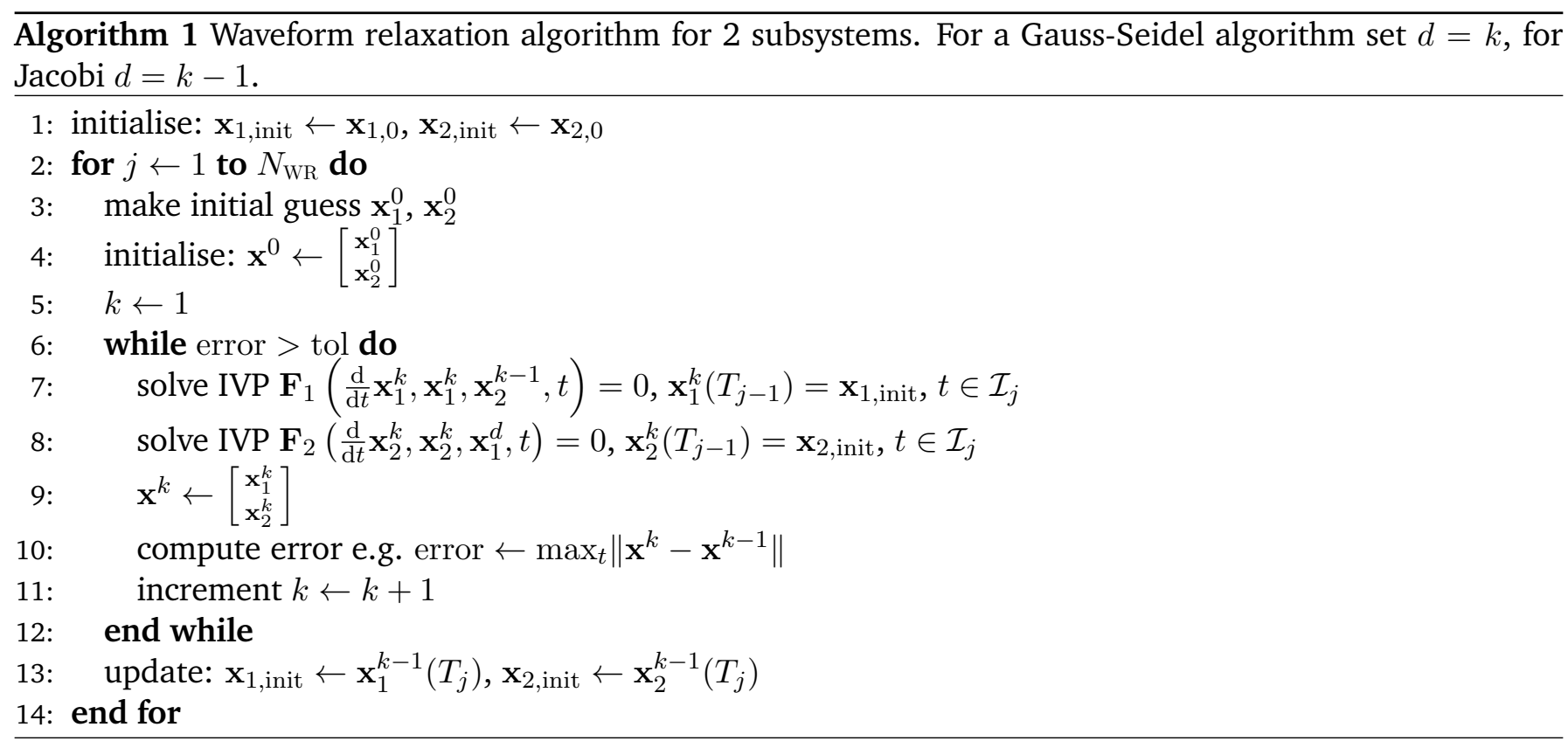

for $t \in \mathcal{I}$ and split into $m=2$ subsystems

$$
\begin{aligned}
\mathbf{F}_{i}\left(\frac{\mathrm{d}}{\mathrm{d} t} \mathbf{x}_{i}, \mathbf{x}_{i}, \mathbf{u}_{i}, t\right) & =0 \\
\mathbf{x}_{i}\left(t_{0}\right) & =\mathbf{x}_{i, 0}
\end{aligned}
$$

with degrees of freedom $\mathbf{x}_{i}$, input $\mathbf{u}_{i}$ and $i=1,2$. WR either of Gauss-Seidel or Jacobi type can be applied to the given splitting of the monolithic system. Algorithm 1 presents the pseudocode of the waveform relaxation algorithm for these two subsystems.

The convergence of WR is known and studied for ODEs and particularly structured DAEs. In [148] it is shown that, for linear ODEs of the type

$$
\mathbf{x}^{\prime}=\mathbf{K} \mathbf{x}+\mathbf{f}(t)
$$

on a time interval of size $H_{\mathrm{WR}}$, the waveform relaxation algorithm converges superlinearly with error bound $\frac{\left(C H_{\mathrm{WR}}\right)^{k}}{k !}$ on the $k$-th WR iteration for a certain constant $C$. The nonlinear case is studied in [143], [149] for ODEs with the structure

$$
\mathbf{A}(\mathbf{x}, \mathbf{u}) \mathbf{x}^{\prime}=\mathbf{f}(\mathbf{x}, \mathbf{u})
$$

with globally invertible mass matrix $\mathbf{A}$ and Lipschitz continuous function $\mathbf{f}$. Again, the algorithm converges superlinearly with error bound

$$
\frac{\left(C_{1} H_{\mathrm{WR}}\right)^{k}}{k !} e^{C_{2} H_{\mathrm{WR}}},
$$

for constants $C_{1}, C_{2}$ [26, Chapter 7]. For a detailed survey on different types of waveform relaxation algorithm and its convergence for ODEs we refer to [26].

In [27]-[30], [33], convergence of the WR algorithm is studied for systems of DAEs. Even for the elemental case of coupled index 1 DAEs that can be written in semi-explicit form

$$
\begin{aligned}
\mathbf{y}^{\prime} & =\mathbf{f}(\mathbf{y}, \mathbf{z}) \\
0 & =\mathbf{g}(\mathbf{y}, \mathbf{z})
\end{aligned}
$$


with nonsingular $\frac{\partial}{\partial \mathbf{z}} \mathbf{g}$, convergence can not be guaranteed in the general case [28]. In [30] different convergence rates are presented for two coupled systems of DAEs depending on their coupling structure.

Theorem 2 (Waveform relaxation convergence order [28]-[30]). Considering a splitting of the index 1 DAE (5.1) into two index 1 systems

$$
\begin{aligned}
\frac{\mathrm{d}}{\mathrm{d} t} \mathbf{y}_{1} & =\mathbf{f}_{1}\left(\mathbf{y}_{1}, \mathbf{z}_{1}, \mathbf{y}_{2}, \mathbf{z}_{2}\right) \\
0 & =\mathbf{g}_{1}\left(\mathbf{y}_{1}, \mathbf{z}_{1}, \mathbf{y}_{2}, \mathbf{z}_{2}\right)
\end{aligned}
$$

$$
\begin{aligned}
\frac{\mathrm{d}}{\mathrm{d} t} \mathbf{y}_{2} & =\mathbf{f}_{2}\left(\mathbf{y}_{1}, \mathbf{z}_{1}, \mathbf{y}_{2}, \mathbf{z}_{2}\right) \\
0 & =\mathbf{g}_{2}\left(\mathbf{y}_{1}, \mathbf{z}_{1}, \mathbf{y}_{2}, \mathbf{z}_{2}\right),
\end{aligned}
$$

then, a Gauss-Seidel waveform relaxation scheme starting with system 1 has convergence rate

- $r=\mathcal{O}\left(H_{\mathrm{WR}}\right)+\alpha$ for fully coupled systems [28], [29].

- $r=\mathcal{O}\left(H_{\mathrm{WR}}\right)$ for couplings with $\frac{\partial \mathbf{g}_{1}}{\partial \mathbf{z}_{2}}=0$ [30].

- $r=\mathcal{O}\left(H_{\mathrm{WR}}^{2}\right)$ for couplings with $\frac{\partial \mathbf{g}_{1}}{\partial \mathbf{y}_{2}}=0, \frac{\partial \mathbf{g}_{1}}{\partial \mathbf{z}_{2}}=0, \frac{\partial \mathbf{g}_{2}}{\partial \mathbf{y}_{1}}=0$ and $\frac{\partial \mathbf{g}_{2}}{\partial \mathbf{z}_{1}}=0$ [30].

Thus, for fully coupled systems, $\alpha<1$ has to be fulfilled for the algorithm to converge. For special cases where the two subsystems have less variable dependencies between each other, linear convergence or even quadratic convergence order can be achieved. Note that e.g. for the case where linear convergence order is achieved in a Gauss-Seidel scheme $\left(\frac{\partial \mathbf{g}_{1}}{\partial \mathbf{z}_{2}}=0\right)$, using Jacobi WR only ensures a convergence rate of $r=\mathcal{O}(\alpha)$ (c.f. [30]). For a more detailed overview of the rigorous convergence analysis see [28]-[30].

Remark 27. More recently, a convergence criterion for index 2 DAE coupled to an ODE has been presented in [150], [151]. For the particular case of an index 2 electric network with LI-cutsets the criterion is given in terms of topological properties of the coupling structure between the element and the circuit. A test example for the criterion can be found in Section 6.2.

\section{Optimised Schwarz waveform relaxation}

Optimised Schwarz methods are domain decomposition methods arising from classical iterative Schwarz methods [37]. The latter are iterative methods to solve Dirichlet boundary value problems by means of dividing the domain into smaller overlapping subdomains, solving them separately and iteratively exchanging information between them to converge to the original problem. In optimised Schwarz methods, these iterative process is expanded, so as to allow the division into non-overlapping domains. Their convergence can still be ensured by means of improving the information exchange, also called the transmission condition. These type of methods optimise the transmission condition so as to ensure the iteration process converges faster.

Intuitively, the optimised Schwarz waveform relaxation methods interpret the WR algorithm as a Schwarz method through time. Applying this to coupled systems arising from different models is sometimes called heterogeneous domain decomposition [152]. In Figure 5.3 a sketch of how the waveform relaxation algorithm of Figure 5.2 can be visualised as a domain decomposition method is given. This new point of view allows the usage of optimised Schwarz techniques to optimise the transmission conditions between the subsystems and hereby converge faster. 


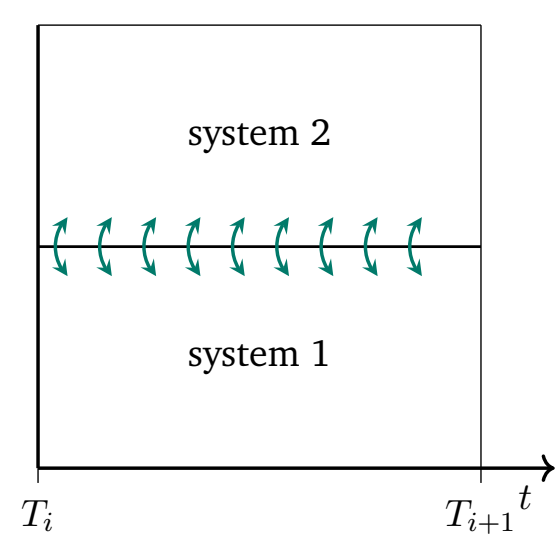

Figure 5.3: Sketch of WR scheme as heterogeneous domain decomposition.

\subsubsection{Optimised Schwarz for accelerator magnets}

In the following we apply the optimised Schwarz waveform relaxation methodology to derive optimised transmission conditions for the co-simulation of magnetoquasistatic field problems and circuits, in particular for the simulation of the LHC quench protection system. However, the idea is bases on Schur complements in frequency domain and can be easily transferred to other cases (see [153]).

The coupled system we consider consists of the two PDEs arising from the heat equation and the eddy current field description due to inter-filament coupling currents. Their semidiscrete systems is afterwards coupled to the DAE modelling the surrounding circuitry. After spatial discretisation of the PDEs (2.40) (2.41), the DAE

$$
\begin{aligned}
\mathbf{K}_{\mathrm{s}}(\mathbf{a}) \frac{\mathrm{d} \mathbf{a}}{\mathrm{d} t}+\mathbf{K}_{\nu}(\mathbf{a}) \mathbf{a}-\mathbf{X}_{\mathrm{s}} \mathbf{i} & =0 \\
\mathbf{v}-\mathbf{X}_{\mathrm{s}}^{\top} \frac{\mathrm{d} \mathbf{a}}{\mathrm{d} t} & =0 \\
\mathbf{M}_{\rho, \mathrm{C}} \frac{\mathrm{d} \mathbf{t}}{\mathrm{d} t}+\mathbf{K}_{\mathrm{k}} \mathbf{t}-\mathbf{q}(\mathbf{a}) & =0 \\
\mathbf{v}_{\mathrm{t}}-\tilde{\mathbf{R}}_{\mathrm{s}}(\mathbf{t}, \mathbf{a}, \mathbf{i}) \mathbf{i} & =0
\end{aligned}
$$

is obtained, with the field system matrices $\mathbf{K}_{\mathrm{s}}:=\mathbf{C}^{\top} \mathbf{M}_{\nu, \tau_{\mathrm{eq}}} \mathbf{C}$ and $\mathbf{K}_{\nu}:=\mathbf{C}^{\top} \mathbf{M}_{\nu} \mathbf{C}$. Here, no classic eddy current effects are modelled and thus $\mathbf{M}_{\sigma}=0$. The heat equation stiffness matrix is $\mathbf{K}_{\mathrm{k}}=\widetilde{\mathbf{S}}^{\top} \mathbf{M}_{\mathrm{k}} \widetilde{\mathbf{S}}$ and $\tilde{\mathbf{R}} \mathrm{s}(\mathbf{t}, \mathbf{a}, \mathbf{i})$ denotes the semidiscrete ohmic resistance on the coils extracted as in (2.44).

The circuit equations arising from flux-charge MNA (2.55) can be described by the DAE

$$
\begin{aligned}
\mathbf{A}_{\mathrm{c}} \frac{\mathrm{d} \mathbf{x}}{\mathrm{d} t}+\mathbf{B}_{\mathrm{c}}(\mathbf{x}) \mathbf{x}+\mathbf{P} \mathbf{i} & =\mathbf{f}(t) \\
\mathbf{P}^{\top} \mathbf{x}-\mathbf{v} & =0 \\
\mathbf{Q}^{\top} \mathbf{x}-\mathbf{v}_{\mathbf{t}} & =0
\end{aligned}
$$

where $\mathbf{x}$ contains the circuit's node potentials, fluxes, charges and the currents across voltage sources and inductances, and $\mathbf{P}$ and $\mathbf{Q}$ are full rank incidence matrices for the field and heat equation systems, respectively. The field and temperature systems are coupled to the circuit in series connection. For a visualisation of their circuit stencil see Figure 5.4. 


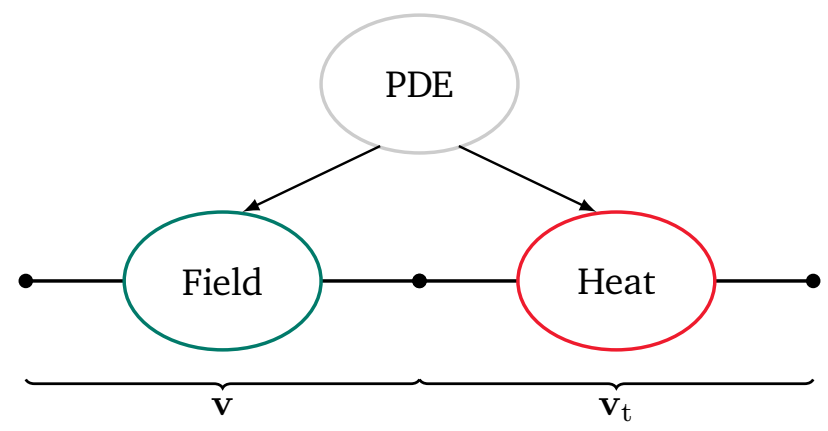

Figure 5.4: Circuit stencil of superconducting magnet and heat equation.

We split the monolithic system (5.2)-(5.3) into two subsystems and simulate them with the waveform relaxation algorithm [3], [56]. The system is split, such that the eddy current together with the heat equation (5.2) form one subsystem ${ }^{1}$ and the circuit DAE (5.3) the other one. We apply the Gauss-Seidel type waveform relaxation and a priori consider the intuitive information exchange of voltages and currents. As it has been shown in Section 4.3, the semidiscrete eddy current equation for inter-filament coupling currents (5.2a)(5.2b) is a resistance-like element and thus there is no preferred voltage or current excitation from the index point of view. We choose current excitation for the field problem and thus obtain the following Gauss-Seidel WR scheme [56] for the $k$-th iteration

System 1 (circuit (5.3))

$$
\begin{aligned}
\mathbf{A}_{\mathrm{c}} \frac{\mathrm{d}}{\mathrm{d} t} \mathbf{x}^{(k+1)}+\mathbf{B}_{\mathrm{c}} \mathbf{x}^{(k+1)}+\mathbf{P i}_{\mathrm{c}}^{(k+1)} & =\mathbf{f}(t) \\
\mathbf{v}_{\mathrm{c}}^{(k+1)}-\mathbf{P}^{\top} \mathbf{x}^{(k+1)} & =0 \\
\mathbf{v}_{\mathrm{t}, \mathrm{c}}^{(k+1)}-\mathbf{Q}^{\top} \mathbf{x}^{(k+1)} & =0
\end{aligned}
$$

with the transmission conditions

$$
\begin{aligned}
& \mathbf{v}_{\mathrm{c}}^{(k+1)}=\mathbf{v}_{\mathrm{m}}^{(k)} \\
& \mathbf{v}_{\mathrm{t}, \mathrm{c}}^{(k+1)}=\mathbf{v}_{\mathrm{t}, \mathrm{m}}^{(k)}
\end{aligned}
$$

System 2 (magnetothermal (5.2))

$$
\begin{aligned}
\mathbf{K}_{\mathrm{s}} \frac{\mathrm{d}}{\mathrm{d} t} \mathbf{a}^{(k+1)}+\mathbf{K}_{\nu} \mathbf{a}^{(k+1)} & =\mathbf{X}_{\mathrm{s}} \mathbf{i}_{\mathrm{m}}^{(k+1)} \\
\mathbf{X}^{\top} \frac{\mathrm{d}}{\mathrm{d} t} \mathbf{a}^{(k+1)} & =\mathbf{v}_{\mathrm{m}}^{(k+1)} \\
\mathbf{M}_{\rho} \frac{\mathrm{d}}{\mathrm{d} t} \mathbf{t}^{(k+1)}+\mathbf{K}_{\mathrm{k}} \mathbf{t}^{(k+1)} & =\mathbf{q}\left(\mathbf{a}^{(k+1)}\right) \\
\mathbf{v}_{\mathrm{t}, \mathrm{m}}^{(k+1)} & =\tilde{\mathbf{R}}_{\mathrm{s}}^{(k+1)} \mathbf{i}_{\mathrm{m}}^{(k+1)}
\end{aligned}
$$

with the transmission condition

$$
\mathbf{i}_{\mathrm{m}}^{(k+1)}=\mathbf{i}_{\mathrm{c}}^{(k+1)} .
$$

Here, $\mathbf{i}_{\mathrm{c}}, \mathbf{v}_{\mathrm{c}}$ and $\mathbf{v}_{\mathrm{t}, \mathrm{c}}$ are degrees of freedom of the circuit's subsystem representing the currents through and voltages across the branches containing field elements and the voltage across the heat equation's branch, respectively. The quantities $\mathbf{i}_{\mathrm{m}}, \mathbf{v}_{\mathrm{m}}$ and $\mathbf{v}_{\mathrm{t}, \mathrm{m}}$ are analogously defined for the magnetothermal subsystem. The simplifying notation $\tilde{\mathbf{R}}_{\mathrm{s}}^{(k)}(t)=\tilde{\mathbf{R}}_{\mathrm{s}}\left(t, \mathbf{a}(t)^{(k)}, \mathbf{i}(t)_{\mathrm{m}}^{(k)}\right)$ is used.

The Gauss-Seidel waveform relaxation algorithm for the magnetothermal-circuit co-simulation with nonoptimised transmission conditions based on [113, Chapter 5] is given in Algorithm 2. Note that for the

\footnotetext{
${ }^{1}$ The choice of coupling the magnetic and thermal systems monolithically is not mandatory. However, in the LHC quench protection circuit examples simulated within STEAM both systems are implemented within the same software (COMSOL) and thus solving them together is the intuitive option.
} 


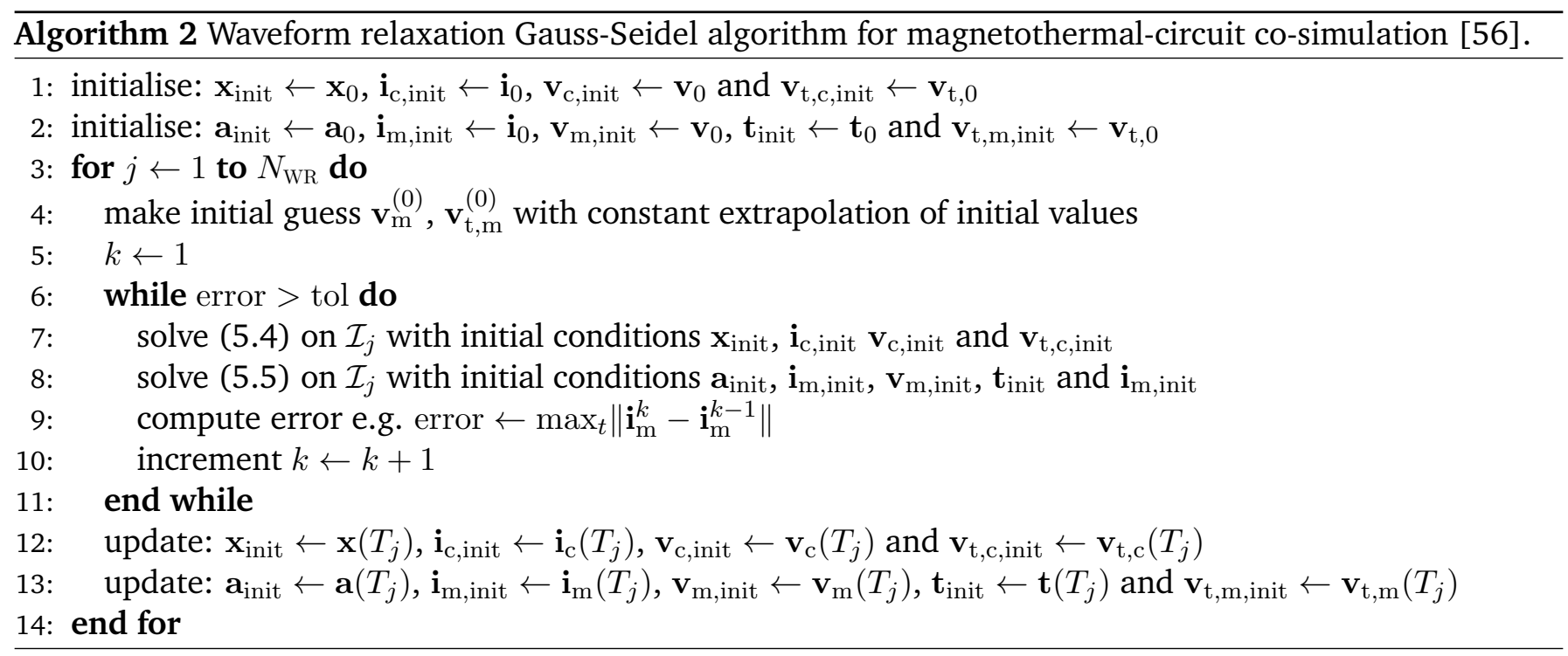

initial guess, constant extrapolation of only the waveforms that are required for the transmission conditions is performed. Higher order extrapolation can also be used which, however, typically yields higher constants in the error bound terms and therefore is commonly not done [28].

As in [39], [40], we interpret the waveform relaxation algorithm as a Schwarz-type method and optimise the transmission conditions (5.4d) and (5.5e) so as to speed up the convergence of the iterations.

\section{Optimisation of the transmission condition}

For the analysis we only consider the co-simulation of the circuit (5.4a)-(5.4b) and the eddy current problem (5.5a)-(5.5b) and optimise only the transmission condition for the circuit (5.4d). For simplicity, we consider settings with only one field element inside the circuit.

The first step that is commonly taken in the optimisation of the transmission condition is to linearise the original system and rewrite it in frequency domain [38], [39]. Here, e.g. for original function $\mathbf{x}=\int_{\mathbb{R}} \hat{\mathbf{x}}(f) e^{2 \pi j f t} \mathrm{~d} f$ and its Fourier transform $\hat{\mathbf{x}}=\int_{\mathbb{R}} \mathbf{x}(t) e^{-2 \pi j f t} \mathrm{~d} t$, then $\frac{\mathrm{d}}{\mathrm{d} t} \mathbf{x}=2 \pi j f \hat{\mathbf{x}}$ and thus with angular frequency $f=2 \pi \omega$ we can rewrite the original system as

$$
\begin{aligned}
\mathbf{A}_{\mathrm{c}} j \omega \mathbf{x}^{(k+1)}+\mathbf{B}_{\mathrm{c}} \mathbf{x}^{(k+1)}+\mathbf{P i}_{\mathrm{c}}^{(k+1)} & =\mathbf{g}(\omega) \\
\mathbf{v}_{\mathrm{c}}^{(k+1)} & =\mathbf{P}^{\top} \mathbf{x}^{(k+1)} \\
\mathbf{v}_{\mathrm{c}}^{(k+1)} & =\mathbf{v}_{\mathrm{m}}^{(k)}
\end{aligned}
$$

and

$$
\begin{aligned}
\mathbf{K}_{\mathrm{s}} j \omega \mathbf{a}^{(k+1)}+\mathbf{K}_{\nu} \mathbf{a}^{(k+1)} & =\mathbf{X} \mathbf{i}_{\mathrm{m}}^{(k+1)} \\
\mathbf{X}^{\top} j \omega \mathbf{a}^{(k+1)} & =\mathbf{v}_{\mathrm{m}}^{(k+1)} \\
\mathbf{i}_{\mathrm{m}}^{(k+1)} & =\mathbf{i}_{\mathrm{c}}^{(k+1)}
\end{aligned}
$$

Note that the degrees of freedom $\mathrm{x}, \mathbf{i}_{\mathrm{c}}, \mathbf{v}_{\mathrm{c}}, \mathbf{a}, \mathbf{i}_{\mathrm{m}}$ and $\mathbf{v}_{\mathrm{m}}$ are now phasors and, formally, require the introduction of new symbols. However, for simplicity of notation we overload the symbols of the original vectors 
as the difference is clear from the context. For the optimisation of the circuit's transmission condition we replace $(5.6 \mathrm{c})$ it by a linear combination

$$
\mathbf{v}_{\mathrm{c}}^{(k+1)}=\alpha \mathbf{i}_{\mathrm{c}}^{(k+1)}-\alpha \mathbf{i}_{\mathrm{m}}^{(k)}+\mathbf{v}_{\mathrm{m}}^{(k)}
$$

and speed up the convergence of the WR iteration by choosing the weighting factor $\alpha$. In the case where the magnetothermal system is coupled to the circuit through several ports and thus the currents and voltages are vectors, $\alpha$ is a matrix.

Following the analysis made in [38], the first step is to make sure that provided the waveform relaxation algorithm has converged, the solution obtained with the classical transmission conditions is the same to the solution obtained with the optimised transmission conditions. This ensures that the fixed point of the WR scheme has not changed.

Proposition 14 (Convergence of classic and generalised transmission condition). If the waveform relaxation algorithm for systems (5.6a)-(5.6a) and (5.7a)-(5.7b) has converged either with transmission conditions (5.6c) and $(5.7 \mathrm{c})$ or $(5.8)$ and $(5.7 \mathrm{c})$, the same solution is obtained.

Proof. Assuming the values obtained at $k \rightarrow \infty$ are written as $\mathbf{v}_{\mathrm{c}}^{\infty}, \mathbf{v}_{\mathrm{m}}^{\infty}, \mathbf{i}_{\mathrm{m}}^{\infty}$ and $\mathbf{i}_{\mathrm{c}}^{\infty}$, the classical WR converges to a solution where

$$
\mathbf{v}_{\mathrm{c}}^{\infty}=\mathbf{v}_{\mathrm{m}}^{\infty} \quad \mathbf{i}_{\mathrm{m}}^{\infty}=\mathbf{i}_{\mathrm{c}}^{\infty},
$$

as here $\mathbf{v}_{\mathrm{m}}^{(k+1)}=\mathbf{v}_{\mathrm{m}}^{(k)}=\mathbf{v}_{\mathrm{m}}^{\infty}$. Also, $\mathbf{i}_{\mathrm{m}}^{(k+1)}=\mathbf{i}_{\mathrm{m}}^{(k)}=\mathbf{i}_{\mathrm{m}}^{\infty}$ and in the optimised case the solution fulfils

$$
\mathbf{v}_{\mathrm{c}}^{\infty}=\alpha \mathbf{i}_{\mathrm{c}}^{\infty}-\alpha \mathbf{i}_{\mathrm{m}}^{\infty}+\mathbf{v}_{\mathrm{m}}^{\infty} \quad \mathbf{i}_{\mathrm{m}}^{\infty}=\mathbf{i}_{\mathrm{c}}^{\infty},
$$

which is equivalent to (5.9) for all $\alpha$.

Now that the correctness of the generalised transmission condition is verified, we can proceed to the optimisation of the contraction factor of the fixed point iteration performed by the waveform relaxation scheme. The convergence of such type of iteration processes can be ensured, whenever the error between two subsequent iterations is contracted (c.f. [26]). To analyse the iteration error, we write a contraction factor $\rho(\alpha)$, such that

$$
\left\|\mathbf{v}_{\mathrm{c}}^{(k+1)}-\mathbf{v}_{\mathrm{c}}^{(k)}\right\|=|\rho(\alpha)|\left\|\mathbf{v}_{\mathrm{c}}^{(k)}-\mathbf{v}_{\mathrm{c}}^{(k-1)}\right\| .
$$

The scheme thus converges if $\rho(\alpha)<1$ and, the smaller the contraction factor, the faster it converges. In particular, for $\rho(\alpha)=0$, the iteration scheme converges immediately [39]. The next step is to find an expression for the contraction factor $\rho(\alpha)$ to compute the value $\alpha$ for which $\rho(\alpha)=0$. As Assumption 18 and Lemma 2 are fulfilled, $\left(\mathbf{K}_{\mathrm{s}} j \omega+\mathbf{K}_{\nu}\right)$ is invertible and we have

$$
\mathbf{v}_{\mathrm{m}}^{(k+1)}=j \omega \mathbf{X}_{\mathrm{s}}^{\top}\left(\mathbf{K}_{\mathrm{s}} j \omega+\mathbf{K}_{\nu}\right)^{-1} \mathbf{X}_{\mathrm{s}} \mathbf{i}_{\mathrm{m}}^{(k+1)} .
$$

Provided there are no cutsets of only current sources and our field element (FI-cutsets), $\left(\mathbf{A}_{\mathrm{c}} j \omega+\mathbf{B}_{\mathrm{c}}\right)$ is invertible. This holds as the system matrices $\mathbf{A}_{c}$ and $\mathbf{B}_{\mathrm{c}}$ are equivalent to the ones that would be obtained from MNA if the branches where field elements are located would contain current sources (c.f. [154]). Therefore, the pencil is regular if no FI-cutsets are given.

Proposition 15 (WR in circuits with FI-cutsets). If the circuit contains cutsets of only current sources and our field element, the waveform relaxation scheme with systems (5.6a)-(5.6a) and (5.7a)-(5.7b) and classic transmission conditions (5.6c) and (5.7c) converges after at most two iterations. 
Proof. If the field element is in a cutset with only current sources $\hat{i}_{1}(j \omega), \ldots, \hat{i}_{n_{\mathrm{c}}}(j \omega)$, then

$$
\mathbf{i}_{\mathrm{c}}^{(k)}+\hat{i}_{1}(j \omega)+\ldots+\hat{i}_{n_{\mathrm{c}}}(t j \omega)=0,
$$

as the sum of the currents in a cutset is zero (KCL). Note that this sum is possible, as we consider settings with only one field element and thus $\mathbf{i}_{\mathrm{c}}^{(k)}$ is a scalar-valued function in frequency domain. Due to the equality, $\mathbf{i}_{\mathrm{c}}^{(k)}$ does not depend on $k$ and converges immediately. Using the transmission condition $(5.7 \mathrm{c}), \mathbf{i}_{\mathrm{m}}^{(k)}$ converges also after the second iteration, whose correct value is propagated to $\mathbf{v}_{\mathrm{m}}^{(k)}$ due to (5.10) and consequently also to $\mathbf{v}_{\mathrm{c}}^{(k)}$.

Using the equality $\mathbf{v}_{\mathrm{c}}^{(k+1)}=\mathbf{v}_{\mathrm{m}}^{(k)}$ and (5.10) in the optimised transmission condition (5.8) and, for circuits without FI-cutsets, we have

$$
\rho(\alpha)=\left(\mathbf{I}+\alpha \mathbf{x}_{\mathrm{P}}^{-1}(\omega)\right)^{-1}(\alpha-\mathbf{Z}(\omega)) \mathbf{x}_{\mathrm{P}}^{-1}(\omega) .
$$

Here, the transfer impedance $\mathbf{x}_{\mathrm{P}}(\omega)=\mathbf{P}^{\top}\left(\mathbf{A}_{\mathrm{c}} j \omega+\mathbf{B}_{\mathrm{c}}\right)^{-1} \mathbf{P}$ is invertible as $\mathbf{P}$ is the incidence vector of the field element and therefore as full column rank. The impedance of the field reads $\mathbf{Z}(\omega)=j \omega \mathbf{X}_{\mathrm{s}}^{\top}\left(\mathbf{K}_{\mathrm{s}} j \omega+\mathbf{K}_{\nu}\right)^{-1} \mathbf{X}_{\mathrm{s}}$.

Optimal convergence of $\rho(\alpha)=0$ is attained for

$$
\alpha=\mathbf{Z}(\omega) .
$$

This value is cheaply computable in frequency domain. However, in time domain, $\mathbf{Z}(\omega)$ becomes more complicated as it includes (even inverted) time derivatives $\left(j \omega \widehat{=} \frac{\mathrm{d}}{\mathrm{d} t}\right)$ that need to be computed. Therefore, finding a simple approximation for it can ease the computation of the optimised transmission condition in time domain, which would allow to apply it even for nonlinear problems.

Remark 28. For circuits with cutsets of only current sources and the field element, the classical waveform relaxation transmission condition is already optimal (see Proposition 15).

\section{Approximation of the optimised transmission condition}

The next step is to approximate the impedance $\mathbf{Z}(\omega)$ by a simpler expression which is easier to compute but still allows a convergence speed up of the WR iterations. Given an invertible $\mathbf{K}_{\nu}$, which is the case in our eddy current for superconducting coils formulation (see Section 4.3.4), we rewrite

$$
\mathbf{Z}(\omega)=j \omega \mathbf{X}^{\top} \mathbf{K}_{\nu}^{-\frac{1}{2}}\left(\mathbf{I}+j \omega \mathbf{K}_{\nu}^{-\frac{1}{2}} \mathbf{K}_{\mathrm{s}} \mathbf{K}_{\nu}^{-\frac{1}{2}}\right)^{-1} \mathbf{K}_{\nu}^{-\frac{1}{2}} \mathbf{X}
$$

with $\mathbf{K}_{\nu}^{-1}=\mathbf{K}_{\nu}^{-\frac{1}{2}} \mathbf{K}_{\nu}^{-\frac{1}{2}}$ For small enough $\omega$ such that

$$
\kappa:=\left\|-j \omega \mathbf{K}_{\nu}^{-\frac{1}{2}} \mathbf{K}_{\mathrm{s}} \mathbf{K}_{\nu}^{-\frac{1}{2}}\right\|<1,
$$

we can use the Neumann series $(\mathbf{I}-\mathbf{T})^{-1}=\sum_{l=0}^{\infty} \mathbf{T}^{l}$ with $\mathbf{T}=-j \omega \mathbf{K}_{\nu}^{-\frac{1}{2}} \mathbf{K}_{\mathrm{s}} \mathbf{K}_{\nu}^{-\frac{1}{2}}$ to obtain

$$
\mathbf{Z}(\omega)=j \omega \mathbf{X}^{\top} \mathbf{K}_{\nu}^{-\frac{1}{2}} \sum_{l=0}^{\infty}\left(-j \omega \mathbf{K}_{\nu}^{-\frac{1}{2}} \mathbf{K}_{\mathrm{s}} \mathbf{K}_{\nu}^{-\frac{1}{2}}\right)^{l} \mathbf{K}_{\nu}^{-\frac{1}{2}} \mathbf{X}
$$


Proposition 16 (Convergence of Neumann Series [56]). The Neumann series (5.11) is convergent for frequencies

$$
f_{\max }<\frac{1}{2 \pi \tau_{\text {eq, } \max }},
$$

with $\tau_{\text {eq, } \max }=\max _{\Omega} \tau_{\text {eq. }}$

Proof. We know the series is convergent for $\kappa<1$ and $\kappa=\left\|-j \omega \mathbf{K}_{\nu}^{-\frac{1}{2}} \mathbf{K}_{\mathrm{s}} \mathbf{K}_{\nu}^{-\frac{1}{2}}\right\|$. Therefore

$$
\kappa \leq \omega_{\max }\left\|\mathbf{K}_{\nu}^{-\frac{1}{2}} \mathbf{K}_{\mathrm{s}} \mathbf{K}_{\nu}^{-\frac{1}{2}}\right\| .
$$

Given a positive semidefinite matrix $\overline{\mathbf{M}}_{\nu, \tau_{\text {eq }}}$ built with the positive semidefinite material $\nu\left(\tau_{\text {eq, } \max }-\tau_{\text {eq }}\right)$ in $\Omega_{\mathrm{s}}$ and $\nu$ in $\Omega_{\mathrm{s}}^{\mathrm{c}}$, we have

$$
\begin{aligned}
\kappa & \leq \omega_{\max }\left\|\mathbf{K}_{\nu}^{-\frac{1}{2}} \mathbf{C}^{\top}\left(\overline{\mathbf{M}}_{\nu, \tau_{\mathrm{eq}}}+\mathbf{M}_{\nu, \tau_{\mathrm{eq}}}\right) \mathbf{C} \mathbf{K}_{\nu}^{-\frac{1}{2}}\right\| \\
& =\omega_{\max }\left\|\tau_{\mathrm{eq}, \max } \mathbf{K}_{\nu}^{-\frac{1}{2}}\left(\mathbf{C}^{\top} \mathbf{M}_{\nu} \mathbf{C}\right) \mathbf{K}_{\nu}^{-\frac{1}{2}}\right\| \\
& \leq \omega_{\max } \tau_{\mathrm{eq}, \max } .
\end{aligned}
$$

Thus, for $\omega_{\max }<\frac{1}{\tau_{\mathrm{eq}, \max }}, \kappa<1$ and the series converges.

Example 5. To exemplify the convergence criterion in terms of $f_{\max }$, we will present real-life values for the time constant $\tau_{\text {eq }}$. It depends on the state of the superconducting cable and its material and is computed as [99]

$$
\tau_{\mathrm{eq}}(B)=\frac{\mu_{0}}{2}\left(\frac{l_{\mathrm{f}}}{2 \pi}\right)^{2} \frac{1}{\left(c_{0}+c_{1} B\right) f_{\mathrm{eff}, \mathrm{x}}},
$$

where $l_{\mathrm{f}}$ is the filament twist-pitch, $\mu_{0}$ the vacuum permeability $f_{\text {eff, }}$ the relative amount of superconductor in the cable matrix and $c_{0}$ and $c_{1}$ depend on the resistivity of the surrounding (copper) matrix. $B$ is the magnitude of the magnetic flux density and, for applications such as the quench simulation of the LHC magnets can be bounded by $0<B \leq 10$.

In [7], some values for these parameters can be found and for the example of a Nb-Ti dipole accelerator magnet we have

$$
\begin{aligned}
& l_{\mathrm{f}}=1.5 \cdot 10^{-2} \mathrm{~m} \text {, } \\
& c_{0}=1.7 \cdot 10^{-10} \Omega \mathrm{m} \\
& c_{1}=4.2 \cdot 10^{-11} \Omega \mathrm{mT}^{-1} \text {, } \\
& f_{\text {eff }, \mathrm{x}}=1 \text {. }
\end{aligned}
$$

Therefore,

$$
\tau_{\text {eq }}<\frac{\mu_{0}}{2}\left(\frac{l_{\mathrm{f}}}{2 \pi}\right)^{2} \frac{1}{c_{0} f_{\mathrm{eff}, \mathrm{x}}}<0.0211 \mathrm{~s},
$$

and the Neumann series is ensured to converge if $f_{\max }<7.5 \mathrm{~Hz}$.

Taking only a finite amount of terms of the Neumann series, we can approximate the optimal $\alpha$. If only the first term is considered, that is $l=0$, then

$$
\mathbf{Z}(\omega) \approx j \omega \mathbf{L}:=j \omega \mathbf{X}^{\top} \mathbf{K}_{\nu}^{-1} \mathbf{X} .
$$


This leads to the optimised transmission condition

$$
\mathbf{v}_{\mathrm{c}}^{(k+1)}=j \omega \mathbf{L} \mathbf{i}_{\mathrm{c}}^{(k+1)}-j \omega \mathbf{L} \mathbf{i}_{\mathrm{m}}^{(k)}+\mathbf{v}_{\mathrm{m}}^{(k)}
$$

which in time domain can be written as

$$
\mathbf{v}_{\mathrm{c}}^{(k+1)}=\frac{\mathrm{d}}{\mathrm{d} t} \mathbf{L i}_{\mathrm{c}}^{(k+1)}-\frac{\mathrm{d}}{\mathrm{d} t} \mathbf{i}_{\mathrm{m}}^{(k)}+\mathbf{v}_{\mathrm{m}}^{(k)}
$$

and corresponds to replacing the field model by an inductance and a correction term on the circuit [56] and was proposed based on engineering intuition in [113], [142]. Extracting the inductance $\mathbf{L}$ is equivalent to solving the magnetostatic system

$$
\begin{array}{r}
\operatorname{curl} \nu \operatorname{curl} \vec{A}=\vec{\chi}_{\mathrm{s}} \mathbf{i} \\
\int_{\Omega} \vec{\chi}_{\mathrm{s}} \cdot \vec{A}=\mathbf{L},
\end{array}
$$

with scalar unit current excitation $\mathbf{i}=1 \mathrm{~A}$. This computation is not too costly and potentially allows saving the higher computational cost that can arise from requiring more WR iterations if no optimised transmission condition is used. Furthermore, the Neumann series leaves the possibility of considering higher order terms and study their influence in the convergence speed up.

Even though the analysis for the optimised transmission condition has been performed for a linear system, it can also be used for nonlinear problems in time domain. It has been previously shown that in practice the optimised coupling improves the convergence of the WR algorithm not only for nonlinear problems, but also for higher frequencies than expected [113, Chapter 6]. We will present the improved algorithm for nonlinear time domain problems in the following section.

\section{Optimised waveform relaxation for nonlinear problems}

For nonlinear problems, that is, PDEs with field-dependent materials, the differential inductance

$$
\mathbf{L}_{\mathrm{d}}=\frac{\partial}{\partial \mathbf{i}} \phi(\mathbf{i})
$$

is required, where $\phi$ is the flux such that $\mathbf{v}=\frac{\mathrm{d}}{\mathrm{d} t} \phi$.

Proposition 17 (Differential inductance matrix). The differential inductance $\mathbf{L}_{\mathrm{d}}$ of a nonlinear magnetostatic problem

$$
\begin{aligned}
& \mathbf{K}_{\nu}(\mathbf{a}) \mathbf{a}=\mathbf{X}_{\mathrm{s}} \mathbf{i} \\
& \mathbf{X}_{\mathrm{s}}^{\top} \mathbf{a}=\boldsymbol{\phi}
\end{aligned}
$$

is constructed with the differential reluctivity matrix as

$$
\mathbf{L}_{\mathrm{d}}=\mathbf{X}_{\mathrm{s}}^{\top}\left(\mathbf{C}^{\top} \mathbf{M}_{\nu, \mathrm{d}}(\mathbf{a}(t)) \mathbf{C}\right)^{-1} \mathbf{X}_{\mathrm{s}}=\mathbf{X}_{\mathrm{s}}^{\top} \mathbf{K}_{\nu, \mathrm{d}}^{-1}(\mathbf{a}(t)) \mathbf{X}_{\mathrm{s}}
$$


Proof. We define the function $\mathbf{F}(\mathbf{a})$ as

$$
\mathbf{F}(\mathbf{a}):=\mathbf{K}_{\nu}(\mathbf{a}) \mathbf{a}=\mathbf{X}_{\mathrm{s}} \mathbf{i}
$$

As it describes a well-posed magnetostatic problem, we know its inverse exists, we call it $\mathbf{F}^{-1}$ and fulfills $\mathbf{a}=\mathbf{F}^{-1}\left(\mathbf{X}_{\mathrm{s}} \mathbf{i}\right)$. Also,

and applying the chain rule yields

$$
\phi=\mathbf{X}_{\mathrm{s}}^{\top} \mathbf{a}=\mathbf{X}_{\mathrm{s}}^{\top} \mathbf{F}^{-1}\left(\mathbf{X}_{\mathrm{s}} \mathbf{i}\right)
$$

$$
\mathbf{L}_{\mathrm{d}}=\frac{\partial}{\partial \mathbf{i}} \boldsymbol{\phi}=\mathbf{X}_{\mathrm{s}}^{\top} \frac{\partial}{\partial \mathbf{y}} \mathbf{F}^{-1}(\mathbf{y})
$$

with $\mathbf{y}=\mathbf{X}_{\mathbf{s}} \mathbf{i}$. Using the inverse function theorem [155, Chapter VII.7],

$$
\mathbf{L}_{\mathrm{d}}=\mathbf{X}_{\mathrm{s}}^{\top}\left(\frac{\mathrm{d}}{\mathrm{d} \mathbf{a}} \mathbf{F}(\mathbf{a})\right)^{-1} \mathbf{X}_{\mathrm{s}}=\mathbf{X}_{\mathrm{s}}^{\top}\left(\frac{\mathrm{d}}{\mathrm{d} \mathbf{a}}\left(\mathbf{K}_{\nu}(\mathbf{a}) \mathbf{a}\right)\right)^{-1} \mathbf{X}_{\mathrm{s}}
$$

and, as it is proven in [113, Appendix A.3] and [70, Chapter 3], $\frac{\mathrm{d}}{\mathrm{d} \mathbf{a}}\left(\mathbf{K}_{\nu}(\mathbf{a}) \mathbf{a}\right)=\mathbf{K}_{\nu, \mathrm{d}}$.

The differential inductance $\mathbf{L}_{\mathrm{d}}$ inherits the time dependence of the magnetic vector potential in the differential reluctivity and in the WR algorithm this yields

$$
\mathbf{L}_{\mathrm{d}}^{(k)}(t):=\mathbf{X}^{\top} \mathbf{K}_{\nu, \mathrm{d}}^{-1}\left(\mathbf{a}^{(k)}(t)\right) \mathbf{X}
$$

In theory, this implies that $\mathbf{L}$ has to be computed and updated in every time step and for every waveform relaxation iteration. However, in [56] a simplified method is implemented, where the differential inductance is computed only on a working point $\mathbf{a}_{\star}$

$$
\mathbf{L}_{\mathrm{d}}=\mathbf{X}^{\top} \mathbf{K}_{\nu, \mathrm{d}}^{-1}\left(\mathbf{a}_{\star}\right) \mathbf{X}
$$

and kept constant for the rest of the algorithm.

Remark 29. Note that, for high nonlinearities, the differential inductance extraction can be extended, as keeping it constant might not suffice. For example it can be be extracted at the beginning of every new window or every $n_{\mathrm{L}, \mathrm{d}}$ time steps. Heuristics methods could be used to decide when to update $\mathbf{L}_{\mathrm{d}}$, provided the WR algorithm requires too many iterations to converge.

The new transmission condition reads

$$
\mathbf{v}_{\mathrm{c}}^{(k+1)}(t)=\mathbf{L}_{\mathrm{d}} \frac{\mathrm{d}}{\mathrm{d} t} \mathbf{i}_{\mathrm{c}}^{(k+1)}(t)+\Delta \mathbf{v}_{\mathrm{m}}^{(k)}(t),
$$

where $\Delta \mathbf{v}_{\mathrm{m}}^{(k)}(t)$ is the correction term, necessary due to the linearisation and the approximation of the Neumann series, and is defined as

$$
\Delta \mathbf{v}_{\mathrm{m}}^{(k)}(t):=\mathbf{v}_{\mathrm{m}}^{(k)}(t)-\mathbf{L}_{\mathrm{d}} \frac{\mathrm{d}}{\mathrm{d} t} \mathbf{i}_{\mathrm{m}}^{(k)}(t)
$$

Based on the optimised transmission condition for the field-circuit coupling, we also update the information exchange between the circuit and the heat equation to exchange resistances instead of voltages [56]. Therefore, (5.4e) is changed to

$$
\mathbf{v}_{\mathrm{t}, \mathrm{c}}^{(k+1)}=\tilde{\mathbf{R}}_{\mathrm{s}}^{(k)}(t) \mathbf{i}_{\mathrm{c}}^{k+1} .
$$

The new, optimised WR algorithm is given in Algorithm 3.

Remark 30. For a system containing several magnets (magnetothermal elements), a mixed Jacobi GaussSeidel approach can be taken, where the circuit is solved first separately (Gauss-Seidel) and field elements are solved in parallel in one iteration (Jacobi). 


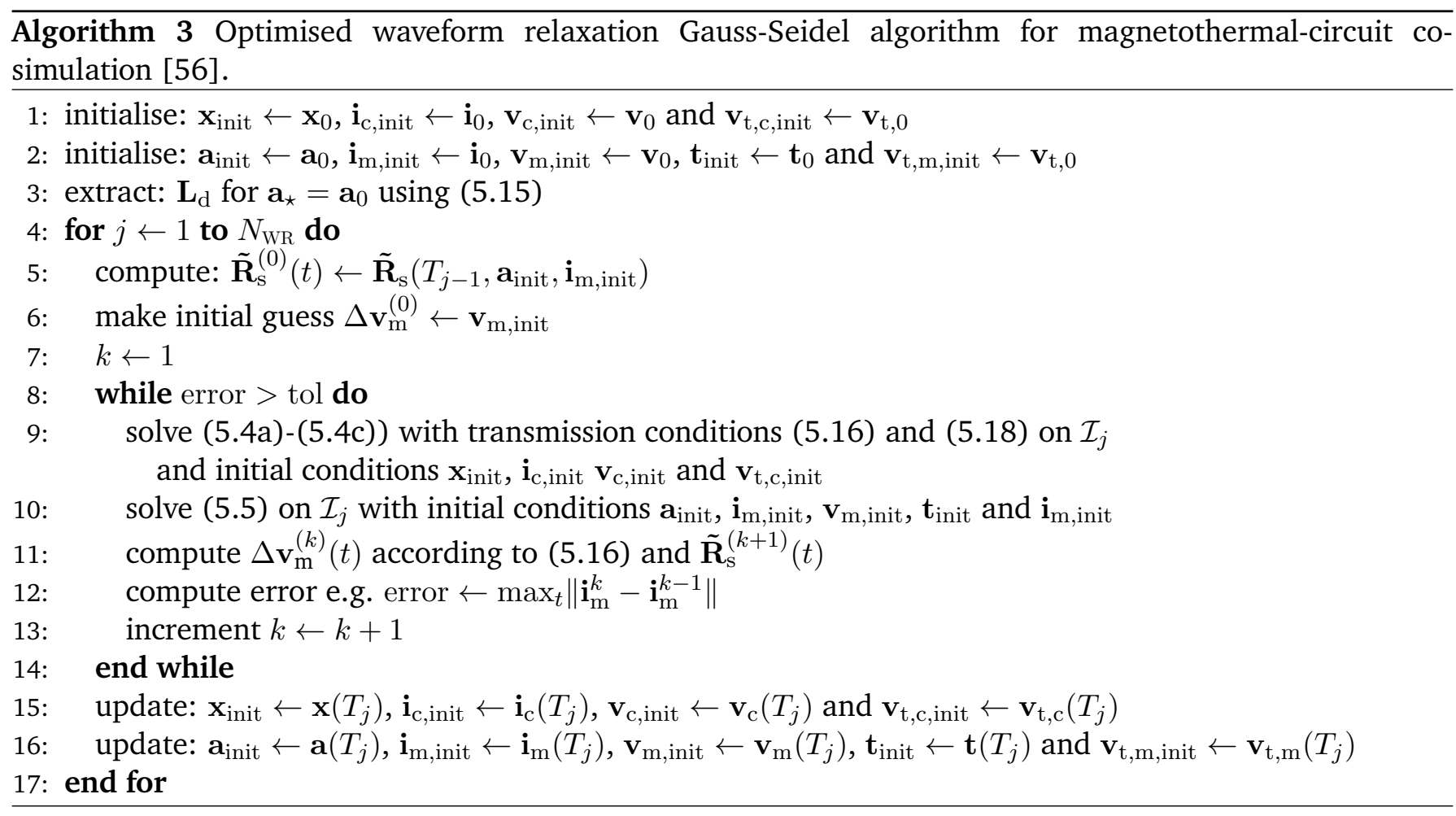

\subsection{Parareal}

Specially coupled multiphysical problems can yield large stiff systems of equations whose simulations on large time windows can be computationally very costly. One way to deal with this inconveniences is the usage of further parallelisation. These exploit the high parallelisation capability of modern computers and computing clusters to execute processes simultaneously and potentially reduce computation time.

Domain decomposition in space [156] are well established and used up to saturation. For further speed up or if time domain simulation yields the bottleneck of computation time, time parallelisation can be a solution. In simulations with large time windows especially arising from systems that require very small time-step sizes such as for example due to pulsed excitations [157] or due to the special physics of the problem [49], parallel-in-time methods are attractive. They allow the speed up of the simulation of initial or boundary value problems arising from time-dependent (ordinary) differential equations. When co-simulation techniques are used, these type of methods, in contrast to domain decomposition methods, are less intrusive and easy to apply, as they can be incorporated into the co-simulation framework.

In the 60s, the need of parallelisation so as to speed up computation time was predicted and in [41], a parallel method for the integration of ODEs is proposed. Rather recently, in 2001 the Parareal method was proposed [42], [43], [45], a parallel-in-time method for initial value problems.

The Parareal algorithm works by dividing the simulation time window into smaller subwindows and solving initial value problems on the subwindows in parallel. Two different solvers for the IVP are defined. First, a computationally cheap solver is chosen, that is used sequentially by the algorithm and is responsible of propagating information between the subwindows. Second, an expensive, accurate solver is required that computes in parallel the solution on the windows. For the algorithm to reduce computation time as much 


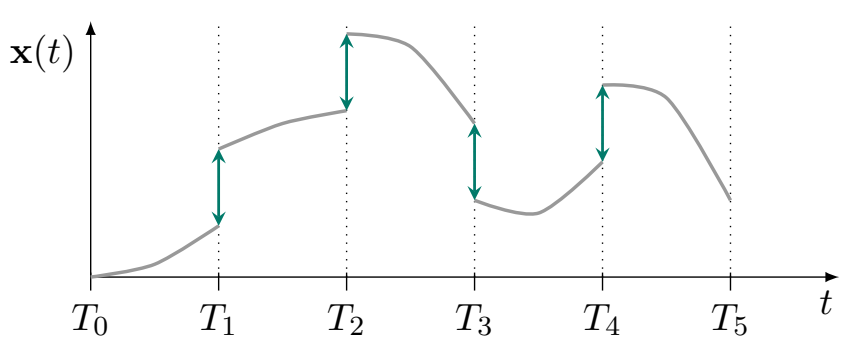

Figure 5.5: Sketch of Parareal jumps across windows.

as possible, the choice of an appropriate cheap solver is of high importance. There are different approaches taken, one of them resulting in the so-called micro-macro Parareal [139].

This work focuses on the usage of Parareal to field-circuit coupled systems. Already in [157], the Parareal algorithm is applied to speed-up time-periodic problems with the example of a circuit with a pulsed excitation. Other works, such as [49], [51] use Parareal on magnetoquasistatic field models of e.g. induction machines. However, unlike in previous work, the special structure of the coupled systems that are considered is exploited to further speed-up computation time.

In the following section we introduce the classic Parareal algorithm as well as the micro-macro version. Both are then studied in the setting of differential algebraic equations.

\subsubsection{Introduction}

For a simulation time window $\mathcal{I}$ and an initial value problem

$$
\begin{aligned}
\mathbf{F}\left(\frac{\mathrm{d}}{\mathrm{d} t} \mathbf{x}, \mathbf{x}, t\right) & =0 \\
\mathbf{x}\left(t_{0}\right) & =\mathbf{x}_{0},
\end{aligned}
$$

with $\mathrm{x}: \mathcal{I} \rightarrow \mathbb{R}^{n_{\mathrm{dof}}}$ and consistent initial condition $\mathrm{x}_{0}, \mathcal{I}$ is divided into $N_{\mathrm{PR}}$ smaller time windows $\mathcal{I}_{n}^{\mathrm{PR}}=$ $\left[T_{n-1}^{\mathrm{PR}} T_{n}^{\mathrm{PR}}\right), n=1, \ldots, N_{\mathrm{PR}}$ with $T_{0}=t_{0}$ of size $\Delta T_{\mathrm{PR}}$. Then, $N_{\mathrm{PR}}$ initial value (sub-)problems

$$
\begin{cases}\mathbf{F}\left(\frac{\mathrm{d}}{\mathrm{d} t} \mathbf{x}, \mathbf{x}, t\right)=0, \quad t \in \mathcal{I}_{1}^{\mathrm{PR}}, & \mathbf{x}\left(T_{0}\right)=\mathbf{X}_{0}, \\ \mathbf{F}\left(\frac{\mathrm{d}}{\mathrm{d} t} \mathbf{x}, \mathbf{x}, t\right)=0, \quad t \in \mathcal{I}_{2}^{\mathrm{PR}}, & \mathbf{x}\left(T_{1}\right)=\mathbf{X}_{1}, \\ \vdots & \\ \mathbf{F}\left(\frac{\mathrm{d}}{\mathrm{d} t} \mathbf{x}, \mathbf{x}, t\right)=0, \quad t \in \mathcal{I}_{N_{\mathrm{PR}}}^{\mathrm{PR}}, & \mathbf{x}\left(T_{N-1}\right)=\mathbf{X}_{N-1},\end{cases}
$$

with $\mathbf{X}_{0}=\mathbf{x}_{0}$, can be solved in parallel, if the initial values (also called interface values) $\mathbf{X}_{0}, \ldots \mathbf{X}_{N-1}$ are known. However, $\mathbf{X}_{1}, \ldots \mathbf{X}_{N-1}$ are a priori unknown to the initial value problem. The aim of Parareal (PR) is to iteratively approximate them so as to converge towards the (correct) continuous solution $\mathrm{x}$ for $t \in \mathcal{I}$. To obtain a continuous solution, the jumps across windows (see Figure 5.5) arising from the unknown (and 
therefore wrong) initial conditions $\mathbf{X}_{1}, \ldots \mathbf{X}_{N_{\mathrm{PR}-1}}$ have to be zero. Therefore, the matching conditions [45]

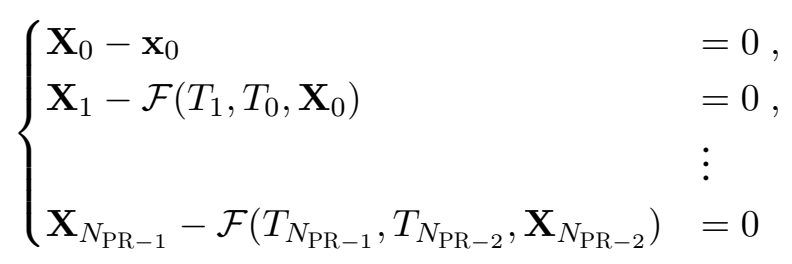

are defined to enforce continuity across the interfaces. Here, $\mathcal{F}$ denotes the solution of the initial value problem (5.19) according to Definition 22.

Definition 22 (Coarse and fine propagators). We define

- $\mathcal{F}\left(t, T_{i}, \mathbf{X}_{i}\right)$ the fine operator, which is the solution at time $t$ of the initial value problem

$$
\mathbf{F}\left(\frac{\mathrm{d}}{\mathrm{d} t} \mathbf{x}, \mathbf{x}, t\right)=0, \quad \quad \quad \quad \quad \mathbf{x}\left(T_{i}\right)=\mathcal{I}_{i+1}^{\mathrm{PR}}, \quad \mathbf{X}_{i} .
$$

This solution is very accurate and assumed to be exact.

- $\mathcal{G}\left(t, T_{i}, \mathbf{X}_{i}\right)$ the coarse operator. This, again, represents the solution at time $t$ of the initial value problem

$$
\mathbf{F}\left(\frac{\mathrm{d}}{\mathrm{d} t} \mathbf{x}, \mathbf{x}, t\right)=0, \quad \quad \quad \quad \quad \mathbf{x}\left(T_{i}\right)=\mathcal{I}_{i+1}^{\mathrm{PR}}, \quad \mathbf{X}_{i},
$$

however is assumed to be computed in a cheap manner and thus less accurate.

The set of matching conditions (5.20) can be visualised as a root finding problem for the unknowns $\mathbf{X}_{1}, \ldots$, $\mathbf{X}_{N_{\mathrm{PR}-1}}$, whose solution is approximated by a Newton method [44] for the (k+1)-th iteration as

$$
\begin{aligned}
& \mathbf{X}_{0}^{k+1}=\mathbf{x}_{0} \\
& \mathbf{X}_{i}^{k+1}=\mathcal{F}\left(T_{i}, T_{i-1}, \mathbf{X}_{i-1}^{k}\right)-\frac{\partial \mathcal{F}}{\partial \mathbf{X}_{i-1}}\left(T_{i}, T_{i-1}, \mathbf{X}_{i-1}^{k}\right)\left(\mathbf{X}_{i-1}^{k+1}-\mathbf{X}_{i-1}^{k}\right), \quad \text { for } i \in 1, \ldots, N_{\mathrm{PR}}-1
\end{aligned}
$$

This idea is well known in the context of multiple shooting method [44]. However, in Parareal, the Jacobian $\frac{\partial \mathcal{F}}{\partial \mathbf{X}_{i-1}}$ of the update formula for the initial values in the Newton scheme is not computed analytically, but approximated with the coarse propagator $\mathcal{G}$ through finite differences [44] as

$$
\frac{\partial \mathcal{F}}{\partial \mathbf{X}_{i-1}}\left(T_{i}, T_{i-1}, \mathbf{X}_{i-1}^{k}\right)\left(\mathbf{X}_{i-1}^{k+1}-\mathbf{X}_{i-1}^{k}\right) \approx \mathcal{G}\left(T_{i}, T_{i-1}, \mathbf{X}_{i-1}^{k+1}\right)-\mathcal{G}\left(T_{i}, T_{i-1}, \mathbf{X}_{i-1}^{k}\right) .
$$

Note that, due to the requirement of the initial condition $\mathbf{X}_{i-1}^{k+1}$ of the $(k+1)$-th Parareal iteration for the first coarse propagation term, this update has to be performed sequentially. However, as the coarse propagator has been chosen to be cheap to compute, this is still computationally cheaper to compute than the original sequential simulation. For a visualisation of the final Parareal algorithm see Algorithm 4.

The convergence of the Parareal algorithm for ODEs is well understood. In [42], [43] convergence rate for linear ODEs is given for certain types of coarse propagators and for bounded and unbounded time intervals, respectively. Later, in [45], the convergence of Parareal is studied for nonlinear IVPs

$$
\frac{\mathrm{d}}{\mathrm{d} t} \mathbf{x}=\mathbf{f}(\mathbf{x}, t), \quad \quad \quad \quad \mathbf{x}\left(t_{0}\right)=\mathbf{x}_{0},
$$




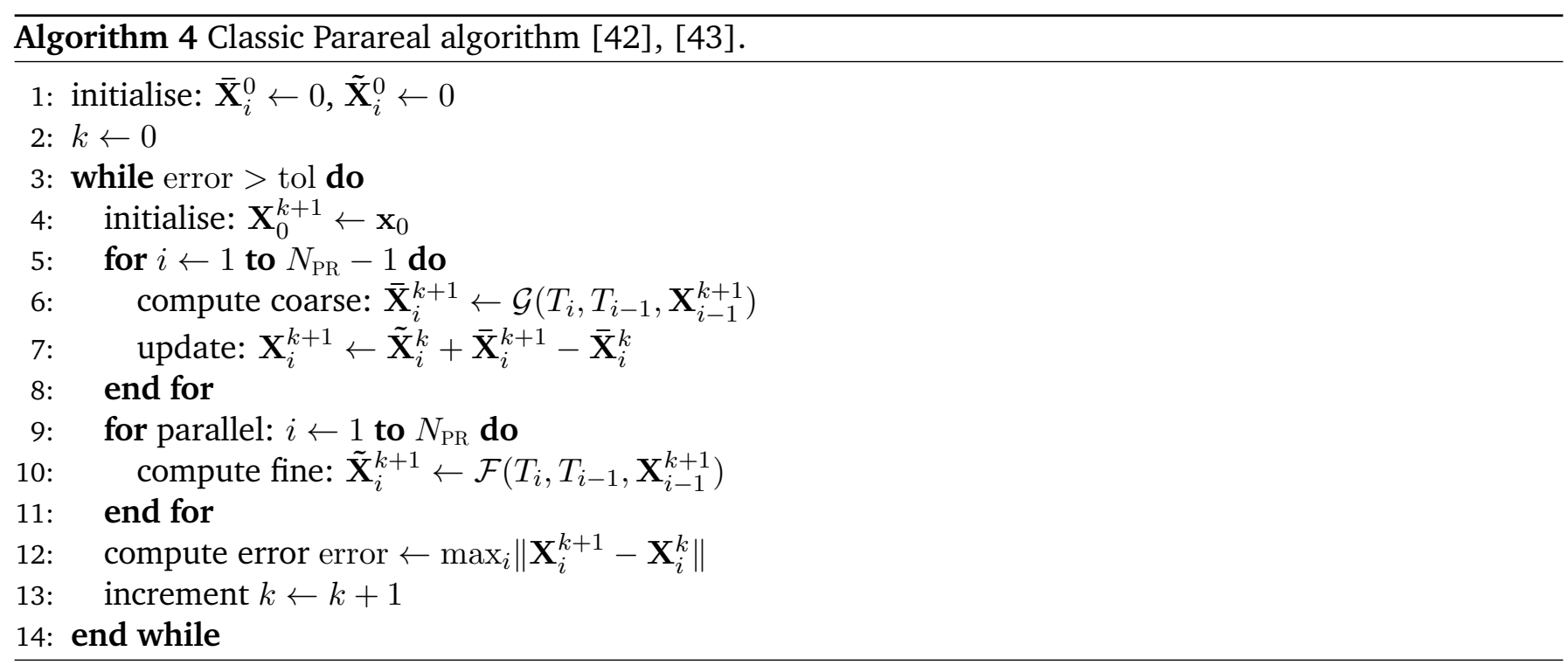

Theorem 3 (Parareal convergence [45]). Given the fine propagator $\mathcal{F}$ which is assumed to solve the IVP exactly and a coarse propagator $\mathcal{G}$ that approximates $\mathcal{F}$ with a local truncation error bound $C_{3} \Delta T_{\mathrm{PR}}^{p+1}$ and satisfies

$$
\mathcal{F}\left(T_{i}, T_{i-1}, t\right)-\mathcal{G}\left(T_{i}, T_{i-1}, t\right)=c_{p+1}(t) \Delta T_{\mathrm{PR}}^{p+1}+c_{p+2}(t) \Delta T_{\mathrm{PR}}^{p+2}+\ldots
$$

Furthermore, for $c_{j}$ continuously differentiable for all $j$ and $\mathcal{G}$ fulfilling

$$
\left\|\mathcal{G}\left(T_{i}, T_{i-1}, t_{1}\right)-\mathcal{G}\left(T_{i}, T_{i-1}, t_{2}\right)\right\| \leq\left(1+C_{2} \Delta T_{\mathrm{PR}}\right)\left\|t_{1}-t_{2}\right\|,
$$

then the error of Parareal on the $k$-th iteration is bounded by

$$
\left\|\mathbf{x}\left(T_{i}\right)-\mathbf{X}_{i}^{k}\right\| \leq \frac{C_{3}}{C_{1}} \frac{\left(C_{1} \Delta T_{\mathrm{PR}}^{p+1}\right)^{k+1}}{(k+1) !}\left(1+C_{2} \Delta T_{\mathrm{PR}}\right)^{i-k-1} \prod_{j=0}^{k}(i-j) .
$$

\section{Micro-macro Parareal}

The choice of the coarse propagator is of utmost importance to ensure a proper speed-up of the algorithm. One typical election is to remain with the same time stepper that is used for the fine solution of the problem $\mathcal{F}$, however using a much bigger time step size [45], [49]. Typically, if implicit Euler is used, the coarse propagator is chosen to perform the implicit Euler method but with a time step size of $\Delta T_{\mathrm{PR}}$, that is, one time step per window.

Other works, such as [50], [139], [158], not only ease the computation of the coarse propagator by changing the time stepper, but also consider a different, simplified problem on the coarse level, which allows to speed up simulation time. In [50], the dynamics of the excitation of the original problem is reduced for the computation of the coarse solution. Therefore, the time domain solution of the original set up is simplified on the coarse level. Convergence for this form of Parareal is shown in the paper. Alternatively, in [139], [158], the (spatial) degrees of freedom of the original model are reduced on the coarse level, which leads smaller system matrices hereby reducing computational cost. This approach is called micro-macro Parareal [139]. 


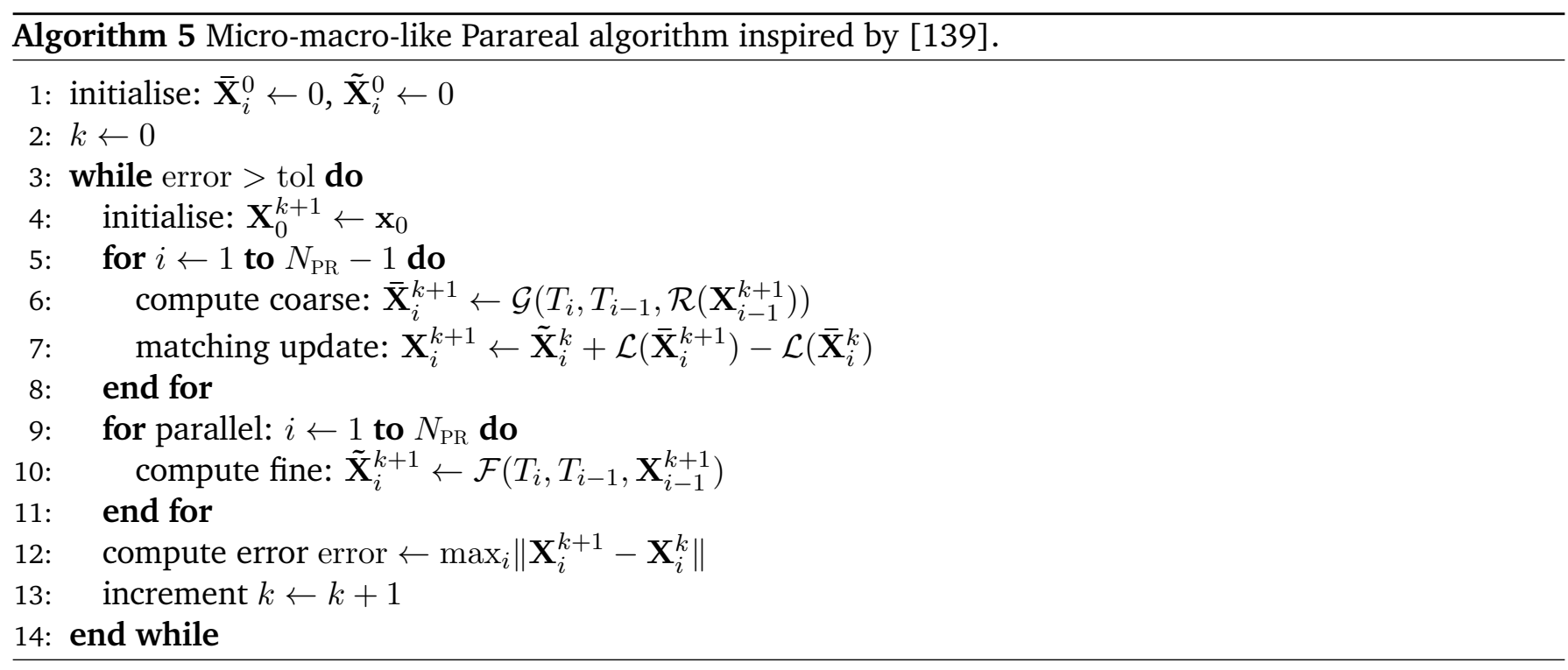

In micro-macro Paraeal, the coarse problem is not defined by the original (fine) IVP (5.19), but an alternative, reduced (coarse) one

$$
\begin{aligned}
\mathbf{F}_{\mathrm{r}}\left(\frac{\mathrm{d}}{\mathrm{d} t} \mathbf{x}_{\mathrm{r}}, \mathbf{x}_{\mathrm{r}}, t\right) & =0 \\
\mathbf{x}_{\mathrm{r}}\left(t_{0}\right) & =\mathbf{x}_{\mathrm{r}, 0},
\end{aligned}
$$

where $\mathbf{x}_{\mathrm{r}}: \mathcal{I} \rightarrow \mathbb{R}^{n_{\mathrm{r}}}$. Here, for example, model order reduction or multirate/multiscale techniques can be used to obtain the reduced model. These methods allow to transform the original system into a nonstiff smaller problem by leaving out the fast dynamics of the original model [139], [158]. As the solution obtained from the coarse propagator $\mathcal{G}$ and the fine propagator $\mathcal{F}$ consist of different degrees of freedom, two additional operators need to be defined. These are reponsible of making a correspondence between the solutions of the coarse and fine models. We denote, inspired by the notation in [139]

- $\mathcal{R}: \mathbb{R}^{n_{\text {dof }}} \rightarrow \mathbb{R}^{n_{\mathrm{r}}}$ the restriction operator that, given $\mathbf{x}_{0}$ an initial value for the original problem (5.19), returns $\mathrm{x}_{\mathrm{r}, 0}$ a valid initial value for the reduced problem (5.21).

- $\mathcal{L}: \mathbb{R}^{n_{\mathrm{r}}} \rightarrow \mathbb{R}^{n_{\mathrm{dof}}}$ the prolongation operator. Here, an initial condition of the coarse problem (5.21) $\mathbf{x}_{\mathrm{r}, 0}$ is transformed into an initial condition for the fine problem (5.19) $\mathbf{x}_{0}$.

These two operators are assumed to be consistent to each other [139], that is,

$$
\mathcal{R}(\mathcal{L}(\mathbf{X}))=\mathbf{X}
$$

Now the original Parareal algorithm in Algorithm 4 can be expanded to a micro-macro Parareal version that contains the prolongation and restriction operator (see Algorithm 5). For alternative micro-macro Parareal algorithms we refer to [139].

\subsubsection{Parareal for differential algebraic equations}

The classic Parareal algorithm and theory is analysed and written for initial value problems arising from ODEs. However, most systems of equations arising from the applications we consider are DAEs. In [48] the 
classic Parareal algorithm is applied to a system of DAEs without a special treatment, however, the correct performance of the algorithm for arbitrary DAEs is not guaranteed. As it has been mentioned in Section 3.3.2, the initial conditions for IVPs arising from DAEs have to be consistent and therefore can not be chosen freely. Especially for higher index DAEs this is not a trivial issue. In Parareal, the initial conditions for the IVPs on the subwindows $\mathcal{I}_{n}^{\mathrm{PR}}$ are computed with the update formula

$$
\mathbf{X}_{i}^{k+1}=\tilde{\mathbf{X}}_{i}^{k}-\overline{\mathbf{X}}_{i}^{k+1}+\overline{\mathbf{X}}_{i}^{k},
$$

with $\tilde{\mathbf{X}}_{i}^{k}:=\mathcal{F}\left(T_{i}, T_{i-1}, \mathbf{X}_{i-1}^{k}\right)$ and $\overline{\mathbf{X}}_{i}^{k}:=\mathcal{G}\left(T_{i}, T_{i-1}, \mathbf{X}_{i-1}^{k}\right)$. This is a sum of solutions of the DAE with different (consistent) initial conditions which are computed with different solvers or time step sizes. For nonlinear problems, it is not ensured that the sum of solutions yields again a valid solution of the problem. This means that even the sum of consistent initial conditions on the update formula could yield an inconsistent initial condition afterwards (see Example 6).

Example 6. To exemplify the possible problematic behaviour in nonlinear systems we consider the index 1 DAE

$$
\begin{aligned}
\frac{\mathrm{d}}{\mathrm{d} t} \mathbf{x} & =\mathbf{x}+\mathbf{y} \\
0 & =\mathbf{x}^{2} \mathbf{y}+\mathbf{y}-\sin (t) .
\end{aligned}
$$

Assuming the consistent initial conditions at $t_{0}=\frac{\pi}{2}$

$$
\mathbf{x}_{0,1}=0, \quad \mathbf{y}_{0,1}=1 \quad \mathbf{x}_{0,2}=-1, \quad \mathbf{y}_{0,2}=\frac{1}{2} \quad \mathbf{x}_{0,3}=1, \quad \mathbf{y}_{0,3}=\frac{1}{2}
$$

and "updating" them up by

$$
\mathbf{x}_{0, \text { new }}=\mathbf{x}_{0,1}-\mathbf{x}_{0,2}+\mathbf{x}_{0,3}=2 \quad \mathbf{y}_{0, \text { new }}=\mathbf{y}_{0,1}-\mathbf{y}_{0,2}+\mathbf{y}_{0,3}=1,
$$

then the obtained initial conditions $\left(\mathbf{x}_{0, \text { new }}, \mathbf{y}_{0, \text { new }}\right)$ are inconsistent.

In [46], [47] a way of coping with this issue is shown for multiple shooting methods and index 2 DAEs. Alternatively, in [49], Parareal is applied to an index 1 DAE with a special structure that ensures that the initial conditions obtained through the update formula are consistent. The rough idea in both cases is to only apply the algorithm on the purely differential components of the DAE, that is, the degrees of freedom that are freely selectable and then compute the consistent algebraic components accordingly. In [49] this is immediately done by the usage of the implicit Euler method as the time integration scheme.

Let us imagine how Parareal could be properly applied to an initial value problem arising from a quasilinear index 2 DAEs of the type (3.31)

$$
\begin{aligned}
\mathbf{A}(\mathbf{x}, t) \frac{\mathrm{d}}{\mathrm{d} t} \mathbf{x}+\mathbf{b}(\mathbf{x}, t) & =0 \\
\mathbf{x}\left(t_{0}\right) & =\mathbf{x}_{0}
\end{aligned}
$$

fulfilling Assumption 12 and with consistent initial condition $\mathrm{x}_{0}$. In e.g. [129], it is shown that the purely differential components of these type of index 2 DAEs can be characterised by the projectors defined for the tractability index in Section 3.3.1. That is, the differential components of the IVP (5.23) are

$$
\mathbf{P P}_{1}(\mathbf{x}, t) \mathbf{x}
$$


and the initial value problem can be uniquely solved if only $\mathbf{P P}_{1}(\mathbf{x}, t) \mathbf{x}$ is prescribed. The rest of the components, that is, $\left(\mathbf{I}-\mathbf{P P}_{1}(\mathbf{x}, t)\right) \mathbf{x}$, are algebraic degrees of freedom of the DAE. They can be consistently computed according to the value of $\mathbf{P P}_{1}(\mathbf{x}, t) \mathbf{x}$ [129]. Formally, the update formula should be applied only to the differential components of the DAE. Therefore, inspired by a micro-macro type Parareal algorithm, we change the classic update (5.22) to

$$
\begin{aligned}
& \hat{\mathbf{X}}_{i}^{k+1}=\mathcal{R}\left(\tilde{\mathbf{X}}_{i}^{k}, T_{i}\right)-\mathcal{R}\left(\overline{\mathbf{X}}_{i}^{k+1}, T_{i}\right)+\mathcal{R}\left(\overline{\mathbf{X}}_{i}^{k}, T_{i}\right), \\
& \mathbf{X}_{i}^{k+1}=\mathcal{L}\left(\hat{\mathbf{X}}_{i}^{k+1}, T_{i}\right),
\end{aligned}
$$

where

- The restriction selects the algebraic components of the solutions as $\mathcal{R}(\mathbf{X}, t)=\mathbf{P P}_{1}(\mathbf{X}, t) \mathbf{X}$.

- The prolongation $\mathcal{L}(\hat{\mathbf{X}}, t)=\mathbf{X}$ computes the consistent algebraic components $\left(\mathbf{I}-\mathbf{P P}_{1}(\hat{\mathbf{X}}, t)\right) \hat{\mathbf{X}}$ such that $\mathbf{P P}_{1}(\mathbf{X}, t) \mathbf{X}=\hat{\mathbf{X}}$ for $\hat{\mathbf{X}}=\mathbf{P P}_{1}(\hat{\mathbf{X}}, t) \hat{\mathbf{X}}$. For detailed information on how consistent initial conditions for some types of DAEs can be computed see [129], [159].

As it can be seen in Algorithm 4, the update formula is computed sequentially. Thus the projection and computation of consistent initial conditions in (5.24) is performed sequentially for all interfaces $T_{i}, i=$ $1, \ldots, N_{\mathrm{PR}}$. If this operation is costly, computational time may be increased. There is software that computes consistent initial conditions for DAEs based on algorithmic differentiation, such as e.g. InitDAE ${ }^{2}$, and thus could be applied for the prolongation operation. However, for black-box models arising e.g. from commercial software, doing this step may not be straightforward, as extracting the system matrices of the DAE and thus knowing its structure is not always possible. In the following we will consider a specific type of DAEs, where, like in [49], the usage of implicit Euler as the time integrator is able to overcome these inconveniences automatically.

\section{Implicit Euler}

In [49] it is shown that for index 1 DAEs arising from magnetoquasistatic curl-curl systems of the type (4.22a), using implicit Euler suffices to obtain consistent initial conditions without the need of changing the original update formula of Parareal (5.22). We will generalise this result to index 2 DAEs with a mild assumption on the structure which is relevant for most application examples we consider.

Before introducing the results, we present some preliminary theoretical definitions. Again we consider quasilinear DAEs as in (5.23) fulfilling Assumption 12.

Assumption 19 (Canonical projector [129, Chapter 1]). We assume the projector $\mathbf{Q}_{1}(\mathbf{y}, \mathbf{x}, t)$ to fulfil

$$
\mathbf{Q}_{1}(\mathbf{y}, \mathbf{x}, t) \mathbf{Q}(t)=0 .
$$

This assumption is also taken in [129, Chapter 1], where it is shown that it can be taken without the loss of generality.

Now we can define the projector $\mathbf{T}(\mathbf{y}, \mathbf{x}, t)$ onto im $\mathbf{Q}(t) \mathbf{Q}_{1}(\mathbf{y}, \mathbf{x}, t)$, which projects onto the index 2 variables that are defined through the hidden constraints [129, Chapter 2.3] and its complementary $\mathbf{U}(\mathbf{y}, \mathbf{x}, t)=$ $\mathbf{I}-\mathbf{T}(\mathbf{y}, \mathbf{x}, t)$.

\footnotetext{
${ }^{2}$ InitDAE is a package for Python that is able to numerically compute the system's index and consistent initial conditions https : //www.mathematik.hu-berlin.de/ lamour/software/python/InitDAE/html/index.html.
} 
Assumption 20 (Index 2 projector [129]). We assume the considered DAE has a smooth only time dependent space im $\mathbf{Q}(t) \mathbf{Q}_{1}(\mathbf{y}, \mathbf{x}, t)$ and thus $\mathbf{T}(t)$ is state independent as well as $\mathbf{U}(t)$. Furthermore, without loss of generality, we choose $\mathbf{T}(t)$ such that [129, Chapter 2.3]

$$
\mathbf{T}(t) \mathbf{P}(t)=0 .
$$

Note that from the definitions of $\mathbf{T}(t)$ and $\mathbf{P}(t)$, also $\mathbf{P}(t) \mathbf{T}(t)=0$ [129, Chapter 2.3].

Assumption 21 (Special structured DAE). We consider index 2 (tractable) DAEs with the structure

$$
\mathbf{A} \frac{\mathrm{d}}{\mathrm{d} t} \mathbf{x}+\mathbf{b}_{1}(\mathbf{U} \mathbf{x}, t)+\mathbf{B}_{2} \mathbf{T} \mathbf{x}=0
$$

and constant projector matrices $\mathbf{U}$ and $\mathbf{T}$. Furthermore, we assume that there exists a constant projector matrix $\mathbf{P}_{1}^{*}$ with $\mathbf{P}_{1}^{*}=\mathbf{I}-\mathbf{Q}_{1}^{*}$ and $\mathbf{Q}_{1}^{*}$ being a constant projector onto im $\mathbf{Q}_{1}(\mathbf{U x}, t)$.

Proposition 18 (Properties of the projectors [129]). Given an index 2 (tractable) DAE with its structure and projectors fulfilling Assumptions 19, 20 and 21, then with $\mathbf{G}_{2}(\mathbf{U x}, t)$ defined as in (3.30),

$$
\mathbf{G}_{2}(\mathbf{U x}, t)=\mathbf{A}+\left(\frac{\partial}{\partial \mathbf{U} \mathbf{x}} \mathbf{b}_{1}(\mathbf{U x}, t) \mathbf{U}+\mathbf{B}_{2} \mathbf{T}\right) \mathbf{Q}+\left(\frac{\partial}{\partial \mathbf{U} \mathbf{x}} \mathbf{b}_{1}(\mathbf{U x}, t) \mathbf{U}+\mathbf{B}_{2} \mathbf{T}\right) \mathbf{P Q}_{1}(\mathbf{U x}, t),
$$

it holds

(a) $\mathbf{P P}_{1}(\mathbf{x}, t) \mathbf{P}=\mathbf{P P}_{1}(\mathbf{x}, t)$.

(b) $\mathbf{U Q P}_{1}(\mathbf{x}, t) \mathbf{P}=0$.

(c) $\mathbf{G}_{2}(\mathbf{U x}, t)^{-1} \mathbf{A}=\mathbf{P}_{1}(\mathbf{U x}, t) \mathbf{P}$.

(d) $\mathbf{G}_{2}(\mathbf{U x}, t)^{-1} \mathbf{B}_{2} \mathbf{T}=\mathbf{T}$.

(e) $\mathbf{P P}_{1}(\mathbf{x}, t)=\mathbf{P P}_{1}(\mathbf{x}, t) \mathbf{P P}_{1}^{*}$.

Proof. Properties $a$ ) and $b$ ) follow from the definitions of the projectors and exploiting Assumptions 19 and 20. Property $c$ ) is shown in [129, Chapter 2.3]. For the Property $d$ ), is suffices to show that $\mathbf{B}_{2} \mathbf{T}=\mathbf{G}_{2}(\mathbf{U x}, t) \mathbf{T}$, which again follows immediately from the definition of the projectors and Assumptions 19 and 20.

The last property exploits Property $a)$ and the fact that projectors $\mathbf{Q}_{1}(\mathbf{x}, t)$ and $\mathbf{Q}_{1}^{*}$ have the same image and thus $\mathbf{P}_{1}(\mathbf{x}, t) \mathbf{P}_{1}^{*}=\mathbf{P}_{1}(\mathbf{x}, t)$ (see e.g. [119, Appendix A.1]).

Proposition 19 (Implicit Euler as prolongation). Given the two initial value problems arising from DAEs with projectors following Assumptions 19, 20 and 21

Inconsistent IC

$$
\begin{aligned}
\mathbf{A} \frac{\mathrm{d}}{\mathrm{d} t} \mathbf{x}+\mathbf{b}_{1}(\mathbf{U x}, t)+\mathbf{B}_{2} \mathbf{T} \mathbf{x} & =0 \\
\mathbf{x}\left(t_{0}\right) & =\mathbf{x}^{0}
\end{aligned}
$$

\section{Consistent IC}

$$
\begin{aligned}
\mathbf{A} \frac{\mathrm{d}}{\mathrm{d} t} \mathbf{x}+\mathbf{b}_{1}(\mathbf{U} \mathbf{x}, t)+\mathbf{B}_{2} \mathbf{T} \mathbf{x} & =0 \\
\mathbf{x}\left(t_{0}\right) & =\mathbf{x}_{0},
\end{aligned}
$$

with inconsistent initial condition $\mathbf{x}^{0}$ and consistent initial condition $\mathbf{x}_{0}$ such that

$$
\mathbf{P} \mathbf{P}_{1}^{*} \mathbf{x}^{0}=\mathbf{P P}_{1}^{*} \mathbf{x}_{0}
$$


then, after at most two implicit Euler steps starting with the inconsistent $\mathbf{x}^{0}$, we obtain a consistent value $\mathrm{x}^{2}$ and

$$
\mathbf{x}^{2}=\mathbf{x}_{2}
$$

where $\mathbf{x}_{2}$ is the solution obtained after two implicit Euler steps with the same stepsize but starting with the consistent IC $\mathbf{x}_{0}$.

Proof. For the proof we follow steps taken in [129, Chapter 2.5] to compute consistent initial values for index 2 DAEs. We start by applying the implicit Euler method to both IVPs

Inconsistent IC

$$
\mathbf{A} \frac{\mathbf{x}^{1}-\mathbf{x}^{0}}{h}+\mathbf{b}_{1}\left(\mathbf{U} \mathbf{x}^{1}, t\right)+\mathbf{B}_{2} \mathbf{T} \mathbf{x}^{1}=0
$$

and multiply them by $\mathbf{G}_{2}\left(\mathbf{U x}^{1}, t\right)^{-1}$. Using Proposition 18.c) the systems are rewritten as

$$
\mathbf{A} \frac{\mathbf{x}_{1}-\mathbf{x}_{0}}{h}+\mathbf{b}_{1}\left(\mathbf{U x}_{1}, t\right)+\mathbf{B}_{2} \mathbf{T} \mathbf{x}_{1}=0
$$

Inconsistent IC

$$
\begin{array}{rlrl}
0= & \mathbf{P}_{1}\left(\mathbf{U x}^{1}, t\right) \mathbf{P} \frac{\mathbf{x}^{1}-\mathbf{x}^{0}}{h}+\mathbf{G}_{2}\left(\mathbf{U x}^{1}, t\right)^{-1} \mathbf{b}_{1}\left(\mathbf{U x}^{1}, t\right) & 0= & \mathbf{P}_{1}\left(\mathbf{U x}_{1}, t\right) \mathbf{P} \frac{\mathbf{x}_{1}-\mathbf{x}_{0}}{h}+\mathbf{G}_{2}\left(\mathbf{U x}_{1}, t\right)^{-1} \mathbf{b}_{1}\left(\mathbf{U x}_{1}, t\right) \\
& +\mathbf{G}_{2}\left(\mathbf{U x}^{1}, t\right)^{-1} \mathbf{B}_{2} \mathbf{T} \mathbf{x}^{1} & +\mathbf{G}_{2}\left(\mathbf{U x}_{1}, t\right)^{-1} \mathbf{B}_{2} \mathbf{T} \mathbf{x}_{1}
\end{array}
$$

As $\mathbf{I}=\mathbf{T}+\mathbf{U}$ and applying Proposition 18.d), we can split the previous equations into

Inconsistent IC

$$
\begin{aligned}
0= & \mathbf{U P}_{1}\left(\mathbf{U} \mathbf{x}^{1}, t\right) \mathbf{P} \frac{\mathbf{x}^{1}-\mathbf{x}^{0}}{h} \\
& +\mathbf{U G}_{2}\left(\mathbf{U} \mathbf{x}^{1}, t\right)^{-1} \mathbf{b}_{1}\left(\mathbf{U} \mathbf{x}^{1}, t\right) \\
0= & \mathbf{T P}_{1}\left(\mathbf{U} \mathbf{x}^{1}, t\right) \mathbf{P} \frac{\mathbf{x}^{1}-\mathbf{x}^{0}}{h} \\
& +\mathbf{T G}_{2}\left(\mathbf{U} \mathbf{x}^{1}, t\right)^{-1} \mathbf{b}_{1}\left(\mathbf{U} \mathbf{x}^{1}, t\right)+\mathbf{T} \mathbf{x}^{1}
\end{aligned}
$$

\section{Consistent IC}

$$
\begin{aligned}
0= & \mathbf{U P}_{1}\left(\mathbf{U x}_{1}, t\right) \mathbf{P} \frac{\mathbf{x}_{1}-\mathbf{x}_{0}}{h} \\
& +\mathbf{U G}_{2}\left(\mathbf{U x}_{1}, t\right)^{-1} \mathbf{b}_{1}\left(\mathbf{U x}_{1}, t\right) \\
0= & \mathbf{T P}_{1}\left(\mathbf{U x}_{1}, t\right) \mathbf{P} \frac{\mathbf{x}_{1}-\mathbf{x}_{0}}{h} \\
& +\mathbf{T G}_{2}\left(\mathbf{U} \mathbf{x}_{1}, t\right)^{-1} \mathbf{b}_{1}\left(\mathbf{U x}_{1}, t\right)+\mathbf{T} \mathbf{x}_{1}
\end{aligned}
$$

The components $\mathbf{U x}$ can be expanded as

$$
\mathbf{U x}=\mathbf{P P}_{1}(\mathbf{U x}, t) \mathbf{x}+\mathbf{P Q}_{1}(\mathbf{U x}, t) \mathbf{x}+\mathbf{U Q x}
$$

(see Proposition 18.b)). Applying this expansion together with Propositions 18.a) and e) and exploiting $\mathbf{P P}_{1}^{*} \mathbf{x}^{0}=\mathbf{P P}_{1}^{*} \mathbf{x}_{0}$, we obtain 
Inconsistent IC

$$
\begin{aligned}
0= & \mathbf{P P}_{1}\left(\mathbf{U} \mathbf{x}^{1}, t\right) \frac{\mathbf{x}^{1}-\mathbf{x}_{0}}{h} \\
& +\mathbf{U G}_{2}\left(\mathbf{U} \mathbf{x}^{1}, t\right)^{-1} \mathbf{b}_{1}\left(\mathbf{U} \mathbf{x}^{1}, t\right) \\
0= & \mathbf{T P}_{1}\left(\mathbf{U} \mathbf{x}^{1}, t\right) \mathbf{P} \mathbf{U} \frac{\mathbf{x}^{1}-\mathbf{x}^{0}}{h} \\
& +\mathbf{T G}_{2}\left(\mathbf{U} \mathbf{x}^{1}, t\right)^{-1} \mathbf{b}_{1}\left(\mathbf{U} \mathbf{x}^{1}, t\right)+\mathbf{T} \mathbf{x}^{1}
\end{aligned}
$$

\section{Consistent IC}

$$
\begin{aligned}
0= & \mathbf{P P}_{1}\left(\mathbf{U x}_{1}, t\right) \frac{\mathbf{x}_{1}-\mathbf{x}_{0}}{h} \\
& +\mathbf{U G}_{2}\left(\mathbf{U x}_{1}, t\right)^{-1} \mathbf{b}_{1}\left(\mathbf{U x}_{1}, t\right) \\
0= & \mathbf{T P}_{1}\left(\mathbf{U x}_{1}, t\right) \mathbf{P} \mathbf{U} \frac{\mathbf{x}_{1}-\mathbf{x}_{0}}{h} \\
& +\mathbf{T G}_{2}\left(\mathbf{U x}_{1}, t\right)^{-1} \mathbf{b}_{1}\left(\mathbf{U x}_{1}, t\right)+\mathbf{T x}_{1}
\end{aligned}
$$

The upper two equations define $\mathbf{U} \mathbf{x}^{1}$ and $\mathbf{U} \mathbf{x}_{1}$ and are the same, as they only depend on $\mathbf{x}_{0}$. Thus $\mathbf{U} \mathbf{x}^{1}=\mathbf{U x}_{1}$. The lower two equations set the values for $\mathbf{T x}^{1}$ and $\mathbf{T} \mathbf{x}_{1}$, which depend on $\mathbf{U x}^{1}, \mathbf{U x}^{0}$ and $\mathbf{U x}_{1}, \mathbf{U x}_{0}$, respectively. We already have that $\mathbf{U x}^{1}=\mathbf{U x}_{1}$. However, if $\mathbf{U} \mathbf{x}^{0}$ is not consistent, $\mathbf{T} \mathbf{x}^{1}$ is also inconsistent and then $\mathbf{T} \mathbf{x}^{1} \neq \mathbf{T} \mathbf{x}_{1}$. Note that if $\mathbf{U x}^{0}=\mathbf{U x}_{0}$, the proof would finish and the correct consistent values are obtained after one Euler step.

For the case where $\mathbf{U x}^{0} \neq \mathbf{U x}_{0}$, the same steps can be applied a second time. This time, $\mathbf{T} \mathbf{x}^{2}$ and $\mathbf{T} \mathbf{x}_{2}$, depend only on $\mathbf{U x}^{1}, \mathbf{U x}^{2}$ and $\mathbf{U x}_{1}, \mathbf{U x}_{2}$, respectively. This time, however, both equations $\mathbf{U x}^{2}=\mathbf{U x}_{2}$, as well as $\mathbf{U x}^{1}=\mathbf{U} \mathbf{x}_{1}$ hold. Therefore, the same solution is obtained for $\mathbf{x}^{2}$ and $\mathbf{x}_{2}$ and the proof is concluded.

In [129, Chapter 2.7] a similar result is shown for DAEs with inconsistent initial condition $\mathbf{x}^{0}$ with consistent variables $\mathbf{U}\left(\mathbf{x}^{0}-\mathbf{x}_{0}\right)=0$ and thus the implicit Euler yields a consistent solution after one step $\mathrm{x}^{1}$. We have generalised the result in [129, Chapter 2.7] to DAEs with only consistent components in $\mathbf{P}\left(\mathbf{x}^{0}-\mathbf{x}_{0}\right)=0$. Furthermore, in contrast to previous results, we have shown that the information obtained from the differential components $\mathbf{P P}_{1}^{*} \mathbf{x}_{0}$ are not lost in the implicit Euler steps. This is the crucial part for Parareal.

Remark 31. In [18] it is shown, that for flux-charge oriented MNA, $\operatorname{im} \mathbf{Q}_{1}(\mathbf{x}, t)$ is constant. Therefore, there exists a constant projector $\mathbf{Q}_{1}^{*}$ onto im $\mathbf{Q}_{1}(\mathbf{x}, t)$. Also, the mass matrix of flux-charge MNA is constant and the index 2 components appear linearly in the system [18], therefore Assumption 21 is fulfilled for these type of systems.

For systems following Assumption 21 (e.g. flux-charge MNA), using implicit Euler as time-stepper for Parareal suffices to guarantee convergence. Similarly to [49], the differential components $\mathbf{P P}_{1}^{*}$ are required to be updated by (5.24). However, restricting the update formula to only apply on this components is not required to be done explicitly in these cases, due to the linearity of $\mathbf{P P}_{1}^{*}$, the regular Parareal update

$$
\begin{aligned}
\mathbf{X}_{i}^{k+1} & =\tilde{\mathbf{X}}_{i}^{k}-\overline{\mathbf{X}}_{i}^{k+1}+\overline{\mathbf{X}}_{i}^{k} \Rightarrow \\
\mathbf{P P}_{1}^{*} \mathbf{X}_{i}^{k+1} & =\mathbf{P P}_{1}^{*} \tilde{\mathbf{X}}_{i}^{k}-\mathbf{P} \mathbf{P}_{1}^{*} \overline{\mathbf{X}}_{i}^{k+1}+\mathbf{P P}_{1}^{*} \overline{\mathbf{X}}_{i}^{k}
\end{aligned}
$$

already updates the differential components correctly. Performing at least two implicit Euler steps on the fine level ensures the solutions obtained in the end to be consistent (see Proposition 19).

\subsection{Parallelised Waveform Relaxation}

In the following we will combine both algorithims of Section 5.1 and Section 5.2 to derive a time parallel waveform relaxation scheme. 
Let us consider a multiphysical setting, whose simulation can not be performed in a monolithical manner and co-simulation techniques are applied to it (see e.g. [5]). For these cases, combining the waveform relaxation algorithm with Parareal allows the speed up of computation time which, as it has been stated before, can be very costly. Here, building the Parareal algorithm on top of the co-simulation framework (e.g. STEAM [5]) does not convey difficulties, as the algorithm is not intrusive.

The combination of both algorithms has been performed previously (see e.g. [48], [52], [53], [160]). On one hand, in [52], [160], the waveform relaxation method is only applied on the fine level, whereas the coarse level remains solving the monolithic system. This methodology, however, can not be used in our context, as we start from the premise that a monolithic simulation is not possible or affordable. On the other hand, [48] uses both for the coarse and fine propagators a waveform relaxation scheme. For both propagators, the amount of WR iterations on the first Parareal iterations is reduced, which allows saving computational cost. This is motivated by the fact that at the beginning of the Parareal algorithm it is not necessary to converge to a very accurate solution even on the fine level. The analysis of this new version of Parareal is not given and, as the convergence proof of Parareal e.g. in [45] does not hold any more, convergence is not ensured here. We take the same approach as [48] and apply the WR algorithm for both propagators, however, in our case with the special motivation of field-circuit coupled systems.

In the following we present the algorithm resulting from our approach of combining waveform relaxation and Parareal (parallelised waveform relaxation), as well as a micro-macro-like version arising from a specific case of parallelised waveform relaxation for field-circuit coupled systems. The content and structure of this section follows [57].

\subsubsection{Waveform relaxation and Parareal}

Let us consider again the initial value problem

$$
\begin{aligned}
\mathbf{F}\left(\frac{\mathrm{d}}{\mathrm{d} t} \mathbf{x}, \mathbf{x}, t\right) & =0 \\
\mathbf{x}\left(t_{0}\right) & =\mathbf{x}_{0},
\end{aligned}
$$

with consistent initial condition $\mathbf{x}_{0}$ and on a time window $\mathcal{I}$. To couple waveform relaxation and Parareal, we start with the choice of setting both time windows to be the same, that is,

$$
\Delta T_{\mathrm{PR}}=\Delta T_{\mathrm{WR}}=\Delta T .
$$

This particular choice is not necessary, but a reasonable one. Thus we partition the simulation time into $N$ time windows $\mathcal{I}_{j}=\left[T_{j-1}, T_{j}\right), j=1, \ldots, N$.

As it has been mentioned before, both the fine propagator $\mathcal{F}$ as well as the coarse propagator $\mathcal{G}$ are chosen to perform the waveform relaxation algorithm on the time windows $\mathcal{I}_{j}$ with a fixed splitting of the monolithic system (5.35) into $m$ subsystems. For a sketch of the algorithm see Figure 5.6. The waveform relaxation scheme on the fine level is iterated until convergence up to a certain tolerance [57], however, on the coarse level the WR scheme only iterates until a fixed amount $k_{\mathrm{c}}$ of iterations.

Remark 32. Note that the combination of the convergence order result of waveform relaxation (Theorem 2) in [30] together with the convergence theorem of Parareal (Theorem 3) in [45] allows obtaining convergence order results for the parallelised waveform relaxation algorithm. The results hold for a coarse propagator $\mathcal{G}$ iterating the WR scheme until $k_{\mathrm{c}}$ iterations and assuming the solution obtained with the internal time 


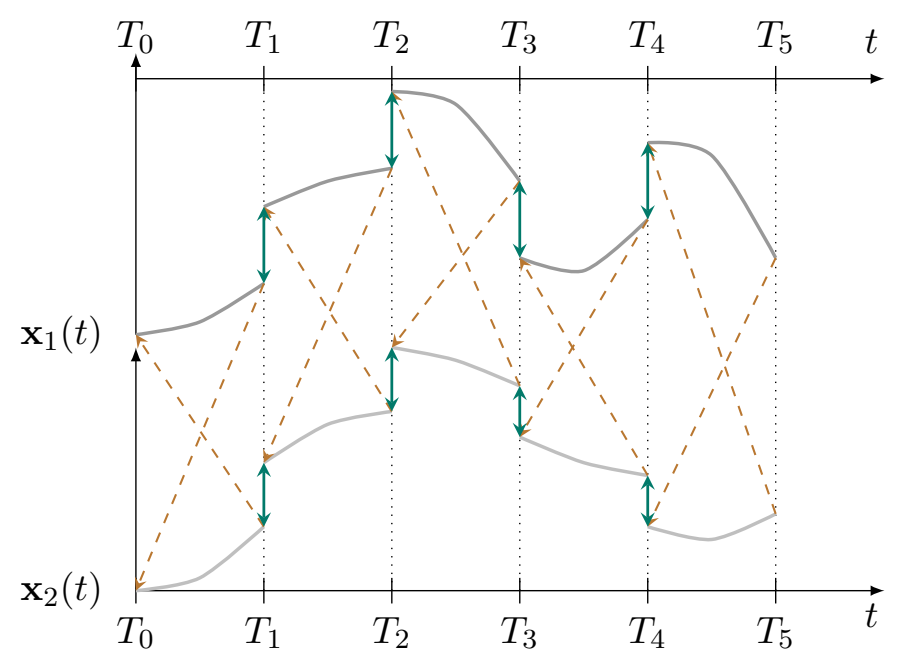

Figure 5.6: Sketch of coupled Parareal and waveform relaxation.

integration scheme of both the coarse and fine solvers is exact. For the splitting of an index 1 DAE into two index 1 systems

$$
\begin{aligned}
\frac{\mathrm{d}}{\mathrm{d} t} \mathbf{y}_{1} & =\mathbf{f}_{1}\left(\mathbf{y}_{1}, \mathbf{z}_{1}, \mathbf{y}_{2}, \mathbf{z}_{2}\right) \\
0 & =\mathbf{g}_{1}\left(\mathbf{y}_{1}, \mathbf{z}_{1}, \mathbf{y}_{2}, \mathbf{z}_{2}\right)
\end{aligned}
$$

$$
\begin{aligned}
\frac{\mathrm{d}}{\mathrm{d} t} \mathbf{y}_{2} & =\mathbf{f}_{2}\left(\mathbf{y}_{1}, \mathbf{z}_{1}, \mathbf{y}_{2}, \mathbf{z}_{2}\right) \\
0 & =\mathbf{g}_{2}\left(\mathbf{y}_{1}, \mathbf{z}_{1}, \mathbf{y}_{2}, \mathbf{z}_{2}\right)
\end{aligned}
$$

the Parareal algorithm (Algorithm 4) after $k$ iterations, neglecting any orders gained through extrapolation, has convergence order bound

- $r=\mathcal{O}\left(\left(H_{\mathrm{WR}}\right)+\alpha\right)^{k+1}$ for fully coupled systems (see Theorem 2).

- $r=\mathcal{O}\left(H_{\mathrm{WR}}^{k+1}\right)$ for couplings with $\frac{\partial \mathbf{g}_{1}}{\partial \mathbf{z}_{2}}=0[30]$.

- $r=\mathcal{O}\left(H_{\mathrm{WR}}^{2(k+1)}\right)$ for couplings with $\frac{\partial \mathbf{g}_{1}}{\partial \mathbf{y}_{2}}=0, \frac{\partial \mathbf{g}_{1}}{\partial \mathbf{z}_{2}}=0, \frac{\partial \mathbf{g}_{2}}{\partial \mathbf{y}_{1}}=0$ and $\frac{\partial \mathbf{g}_{2}}{\partial \mathbf{z}_{1}}=0$ [30].

There are different ways of reducing the computational cost in the given algorithm. One straightforward idea, also considered in [48], is to use time steps of $\Delta T$ on the coarse level for the time integration inside the WR algorithm. Furthermore, in [48] two more strategies are presented to speed up the simulation time of the fine propagator. First, as it has been mentioned above, the amount of WR iterations is not only reduced on the coarse propagator, but also on the fine one during the first Parareal iterations. Second, a methodology is presented to improve the initial guess of WR. Starting from the second Parareal iteration, no constant extrapolation is performed for the initial guess, but the solution of the previous iteration is shifted to the new initial conditions as initial guess. These changes are not covered by Theorem 3 .

In the following, we present a problem specific methodology to speed up the simulation on the coarse level for the special case of field-circuit coupled systems [57]. Its main idea is to disregard the time dynamic part of the PDE and through Schur complement reduce the degrees of freedom on the coarse level. 


\section{Micro-macro for field-circuit coupled systems}

The proposed method is applied to field-circuit coupled systems arising from eddy current curl-curl equations

$$
\begin{aligned}
\mathbf{M} \frac{\mathrm{d}}{\mathrm{d} t} \mathbf{a}+\mathbf{K}_{\nu} \mathbf{a} & =\mathbf{X}_{\mathrm{s}} \mathbf{i} \\
\mathbf{X}_{\mathrm{s}}^{\top} \frac{\mathrm{d}}{\mathrm{d} t} \mathbf{a} & =\mathbf{v}
\end{aligned}
$$

coupled to circuits (5.3a)-(5.3a), where the eddy current effects are represented by the term $\mathbf{j}_{\text {eddy }}=\mathbf{M} \frac{\mathrm{d}}{\mathrm{d} t} \mathbf{a}$. These eddy current effects can arise from different models such as the classical conductivity matrix of the $A^{*}$ eddy current PDE (2.20), the superconducting inter-filament coupling currents model (2.40) or lamination models such as e.g. the one presented in [161].

To reduce computational cost of the Parareal algorithm only the coarse model is modified, that is, the fine propagator is kept as the WR algorithm until convergence applied to the coupled system (5.37),(5.3a)-(5.3a). However, on the coarse level, waveform relaxation is applied with $k_{\mathrm{c}}=\frac{1}{2}$ iterations, constant extrapolation as initial guess and with optimised transmission condition (5.14). Here, $k_{\mathrm{c}}=\frac{1}{2}$ denotes performing only half a WR iteration, that is, only solving the first system of the Gauss-Seidel scheme. In practice this yields the reduced coarse system

$$
\begin{aligned}
\mathbf{A}_{\mathrm{c}} \frac{\mathrm{d} \mathbf{x}}{\mathrm{d} t}+\mathbf{B}_{\mathrm{c}}(\mathbf{x}) \mathbf{x}+\mathbf{P} \mathbf{i}_{\mathrm{c}} & =\mathbf{f}(t) \\
\mathbf{P}^{\top} \mathbf{x}-\mathbf{v}_{\mathrm{c}} & =0 \\
\mathbf{v}-\frac{\mathrm{d}}{\mathrm{d} t} \mathbf{L} \mathbf{i}_{\mathrm{c}} & =0,
\end{aligned}
$$

which corresponds to replacing the field by an inductance $\mathbf{L}=\mathbf{X}_{\mathrm{s}}^{\top} \mathbf{K}_{\nu}^{+} \mathbf{X}_{\mathrm{s}}$ on the circuit side. Here, the matrix $\mathbf{K}_{\nu}^{+}$denotes the pseudoinverse. See Figure 5.7 for a sketch of the coarse and fine systems. Note that this corresponds to neglecting the eddy current effects of the original system (5.37) on the coarse model.

This methodology can be interpreted as a micro-macro-like Parareal algorithm, as, for the coarse model, the degrees of freedom of original problem are reduced to only contain the circuit's variables. That is, the semidiscrete magnetic vector potential arising from space discretisation, that is, the degrees of freedom of the field element are not considered. This can substantially reduce the computational cost, as a much smaller system is computed on the sequential coarse propagator.

The update operation requires, like in the micro-macro algorithm (see Algorithm 5), restriction and prolongation operators to exchange information between the coarse and fine systems. The degrees of freedom of the fine model obtained from applying the WR scheme to the field-circuit coupled system are

$$
\mathbf{x}_{\mathrm{f}}^{\top}=\left(\mathbf{a}^{\top}, \mathbf{i}_{\mathrm{m}}^{\top}, \mathbf{v}_{\mathrm{m}}^{\top}, \mathbf{x}^{\top}, \mathbf{i}_{\mathrm{c}}^{\top}, \mathbf{v}_{\mathrm{c}}^{\top}\right),
$$

whereas the coarse solver operates only with variables

$$
\mathbf{x}_{\mathrm{c}}^{\top}=\left(\mathbf{x}^{\top}, \mathbf{i}_{\mathrm{c}}^{\top}, \mathbf{v}_{\mathrm{c}}^{\top}\right) \text {. }
$$

Thus, the restriction operator simply reduces the degrees of freedom and is defined as

$$
\mathcal{R}\left(\mathbf{x}_{\mathrm{f}}\right):=\left(\mathbf{x}^{\top}, \mathbf{i}_{\mathrm{c}}^{\top}, \mathbf{v}_{\mathrm{c}}^{\top}\right) .
$$



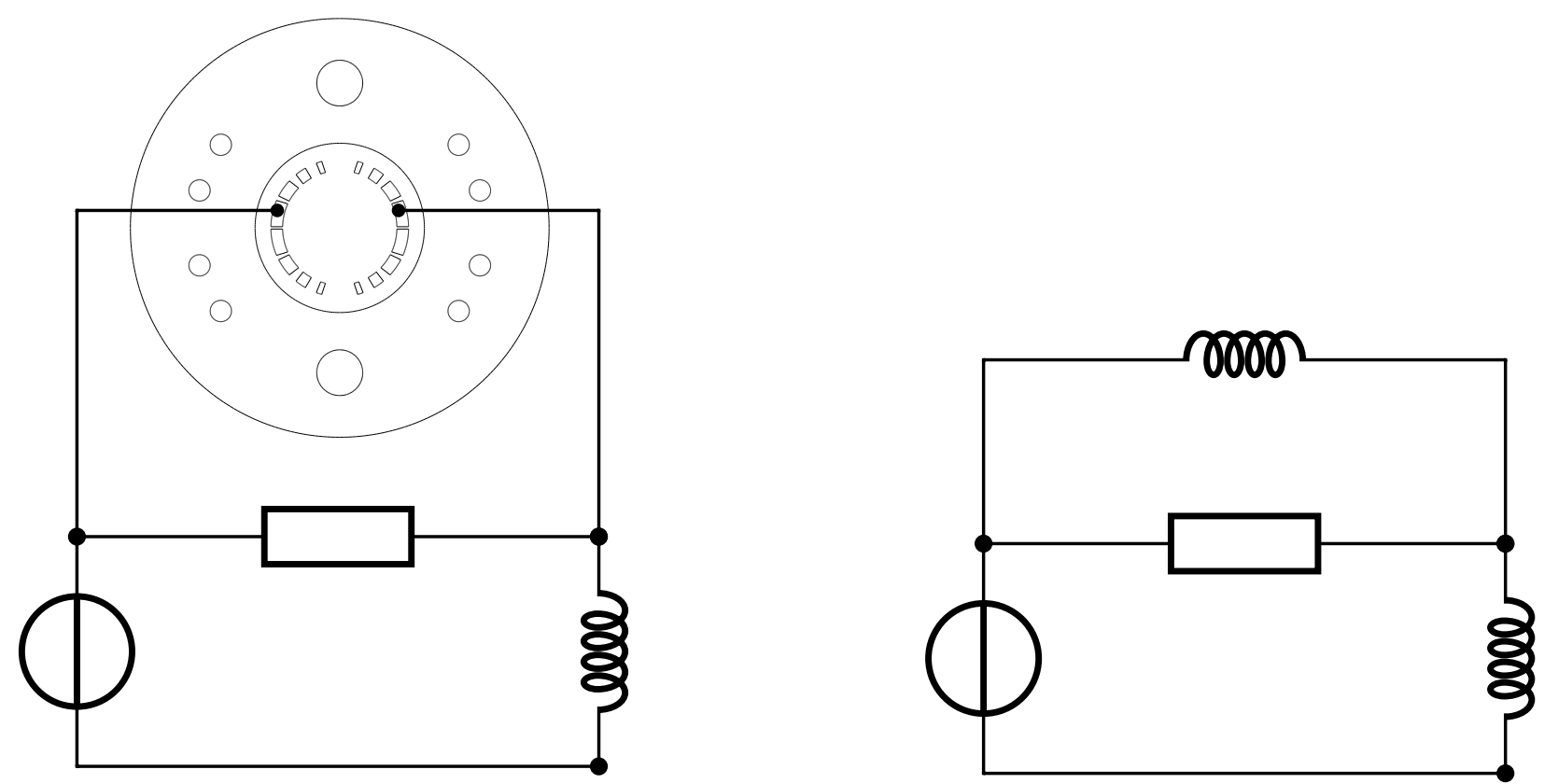

Figure 5.7: Sketch of fine (left) and coarse (right) models for parareal applied to a field-circuit coupled problem example.

The prolongation is obtained by solving the magnetostatic approximation of the original problem, that is, we compute $\mathbf{a}_{\text {init }}$ such that

$$
\mathbf{K}_{\nu} \mathbf{a}_{\text {init }}=\mathbf{X}_{\mathrm{s}} \mathbf{i}_{\mathrm{c}}
$$

Then, the operator returns

$$
\mathcal{L}\left(\mathbf{x}_{\mathrm{c}}\right):=\left(\mathbf{a}_{\text {init }}^{\top}, \mathbf{i}_{\mathrm{c}}^{\top}, \mathbf{v}_{\mathrm{c}}^{\top}, \mathbf{x}^{\top}, \mathbf{i}_{\mathrm{c}}^{\top}, \mathbf{v}_{\mathrm{c}}^{\top}\right)^{\top}
$$

The update is performed as in Algorithm 5. Note that using this matching update and with the prolongation and restriction operators as defined above, after the $k$-th Parareal iteration, the solution at the first $k$ intervals is exact (proof follows analogous to [139, Section 4.3.1]). This property is fulfilled by the original Parareal algorithm and also in our micro-macro like field-circuit Parareal version.

Remark 33. Note that the prolongation operator neglects the eddy current effects. This does not only affect the voltage-to-current relation on the circuit level (in time), but also disregards the skin effect of the magnetic vector potential on the field side (in space). If this effect is very prominent, this may affect the speed of convergence of the Parareal algorithm. Therefore, for problems with large eddy current effects, the prolongation might have to be improved to be able to capture the different field distribution due to the skin effect.

\subsection{Conclusions}

In this chapter, two iterative methods for time domain simulation have been presented. Their study is mainly inspired by systems arising from field-circuit coupling, however they can be generalised to other type of coupled systems. 
The first part has presented co-simulation techniques for multiphysical system by means of the waveform relaxation algorithm. It has focused on field-circuit coupled systems for quench simulation, whose co-simulation has been interpreted by means of optimised Schwarz methods. This has allowed the computation of an optimal information exchange in the WR iterations that leads to faster convergence. By means of Neumann series, the transmission condition could be approximated for small frequencies. The optimised transmission condition has been physically interpreted as plugging in the magnetoquasistatic field as an inductance with a correction term into the circuit, which was already proposed in [113]. Furthermore, in contrast to previous work, not using numerical optimisation to find the optimised information exchange, allows taking higher order terms of the series to further improve convergence speed in a future work. The Schur-complement procedure and later series approximation can easily be extended to other systems. For example, later in [153], the same methodology is used to extract equivalent circuit approximations for high termperature superconducting magnets described with mixed formulations.

The second part has discussed the Parareal algorithm for computation time speed up by means of time parallelisation. The algorithm has been studied in the context of DAEs by discussing possible inconveniences that may arise when applying it to higher index DAEs. A possible extension of the algorithm to circumvent the difficulties has been presented and, for special structured DAEs such as for example the ones arising from flux-charge MNA, it has been shown that implicit Euler can correct the complications that Parareal can have when applied to DAEs.

In the last part, waveform relaxation and Parareal have been combined with the special focus on simulating field-circuit coupled systems. One already known combination of both algorithms has been presented and a special approach has been taken that yields a micro-macro-like Parareal algorithm. In the micro-macro version, the coarse model considered the eddy current model only as an inductance inside the circuit, whereas the fine models solved the entire coupled system by means of waveform relaxation. Therefore, behaviour arising from eddy currents such as the skin effect could not be described by the coarse simplification. Models with large eddy current effects might require modifications of the presented algorithm. This leaves room for a future study on how to capture the required behaviour without increasing the computational cost on the coarse level too much. 


\section{Numerical Examples}

In this chapter the theoretical results presented in Chapters 4 and 5 are verified by means of numerical test examples.

Section 6.1 presents the time domain simulation of two field models that are classified with the generalised circuit elements defined in Chapter 4. The first one corresponds to an inductance-like and the second one to a capacitance-like element. Both are monolithically coupled to two different circuits, one yielding an index 1 and the other one an index 2 DAE, according to the Theorem presented in Section 4.2, to analyse their sensitivity towards small, high frequent perturbations. The simulation of the inductance-like element is taken from [55].

Section 6.2 applies the waveform relaxation method to an index 2 circuit containing LI-cutsets coupled to an eddy current equation solved on a transformer model. Two coupling approaches are made which, according to the theory of [150] yield a convergent and a divergent iteration scheme. The simulation results arise from [151].

In Section 6.3 the optimised waveform relaxation algorithm of Section 5.1 is applied to a field-circuit coupled system consisting of an accelerator magnet with its surrounding circuitry. The simulation results are taken from [56].

Section 6.4 shows the evolution of the Parareal algorithm applied to two nonlinear index 2 circuits described with flux-charge MNA. Here, the effectiveness of performing the numerical integration with implicit Euler inside Parareal as the information propagator for the differential components of the DAE is tested (the theoretical results of Section 5.2).

Finally, Section 6.5 applies the two algorithms combining Parareal and waveform relaxation of Section 5.3 to a linear two dimensional transformer model surrounded by a nonlinear circuit. These simulation results are taken from [24].

\subsection{DAE Index of Refined Models}

To illustrate the effects of the higher index of DAE arising from refined models in circuits and hereby verify the theoretical results of Chapter 4, two different field models described with their corresponding approximation of Maxwell's equations are simulated. The first model, corresponding to an inductance-like element arises from an eddy current formulation, whereas the second one, classified as a capacitance-like element, is described by an electroquasistatic setting. As it has been proven in Theorem 1, given the correct classification of the refined element, the index of the coupled problem only depends on the topology of the surrounding circuit. Therefore, for each one of the examples we consider two different circuit topologies that yield DAEs with different index. All variants are then first simulated with a given excitation and 


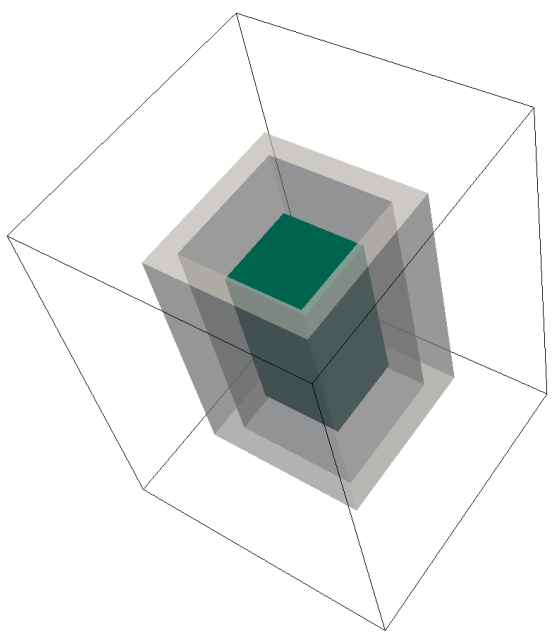

(a) Squared coil in transparent grey with aluminium core in cyan.

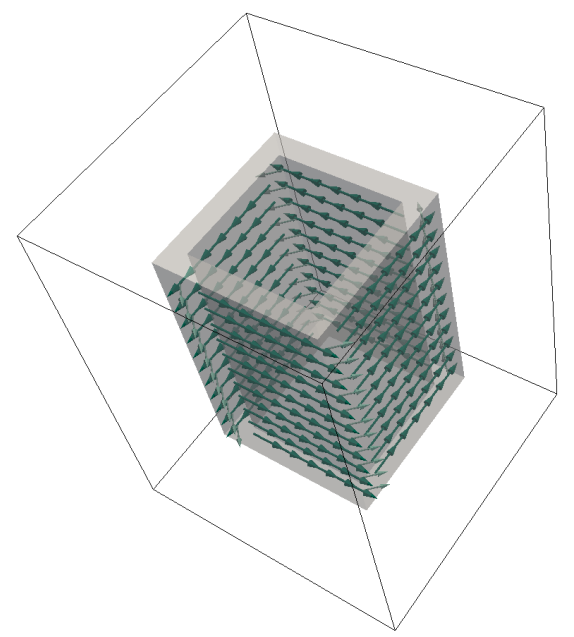

(b) Semidiscrete source current density $\mathbf{j}_{\mathrm{s}}=\mathbf{C} \mathbf{Y}_{\mathrm{s}}$.

Figure 6.1: Inductance-like element example's geometry [55], [58].

then with a small, high frequent perturbation to see how in the perturbation-sensitive index 2 cases this is magnified.

\subsubsection{Inductance-like element}

In Section 4.3.2, two different semidiscrete magnetoquasistatic field formulations are classified as inductancelike elements. The first one corresponds to an $\vec{A}^{*}$ formulation (see Proposition 4), whose topological index interpretation when coupled to circuits confirms already known results. A numerical test example to verify the theoretical results for this type of refined model is already given in [22] and therefore no further numerical models are presented here.

The second inductance-like element arises from the $\vec{T}-\Omega$ formulation. This new result is corroborated by means of a model example consisting of a square coil with an aluminium core (see Figure 6.1), which is coupled to a surrounding circuit [55], [58]. For a more detailed description of the dimensions and properties of the model see $[58]^{1}$. The spatial discretisation of the $\vec{T}-\Omega$ PDE on the coil is performed with the finite integration technique and, for regularisation, a tree-cotree gauging is applied to the semidiscrete electric vector potential $t$. This is carried out with an in-house code and yields a system of DAEs with 4442 unknowns that, due to Proposition 7 is classified as an inductance-like element.

For the numerical test, the next step is to couple the element to a surrounding circuit. Following Theorem 1, an index $2 \mathrm{DAE}$ is obtained if the element is located in a cutset consisting of only current sources and inductance-like elements ( $\mathrm{I} \mu$-cutset). If no such cutset is given (and no loops of capacitance-like elements with voltage sources are present), the field-circuit coupled system has index 1 . Thus we build two different circuits around the square coil and in one of them the coil is located in a cutset with a current source (I $\mu$ cutset). Let us consider the simplest possible circuits that fulfil the requirements. For the first example, the inductance-like element is coupled to a voltage source $v_{\mathrm{S}}(t)$ (see Figure 6.2a). Clearly, this circuit does not

\footnotetext{
${ }^{1}$ The code containing the system matrices of the square coil model can be found in https ://github.com/temf/daes_in_cem
} 


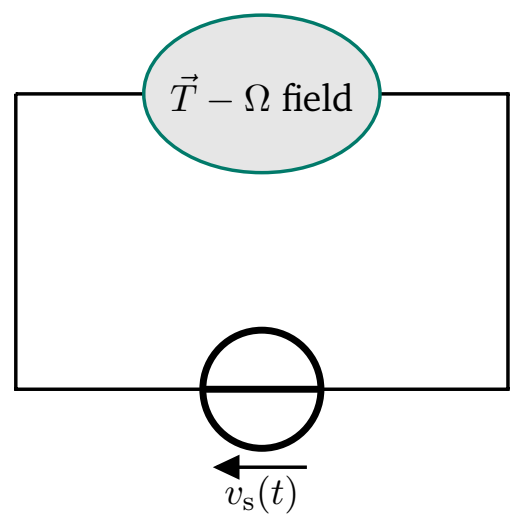

(a) Index 1 circuit with voltage source $v_{\mathrm{s}}(t)$.

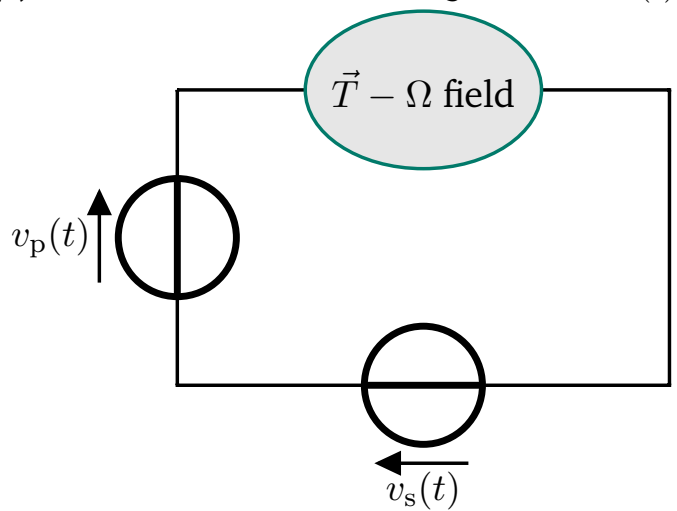

(c) Index 1 circuit with voltage source $v_{\mathrm{s}}(t)$ and perturbation $v_{\mathrm{p}}(t)$.

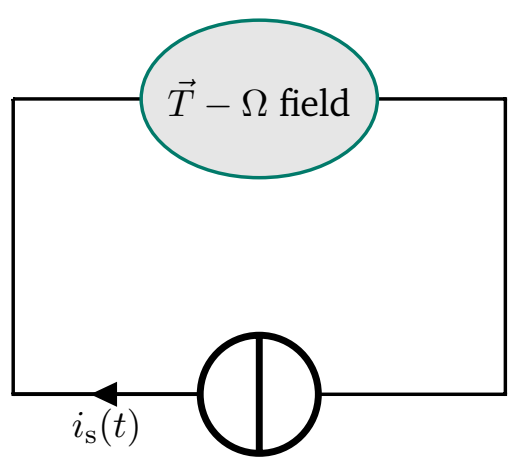

(b) Index 2 circuit with current source $i_{\mathrm{s}}(t)$.

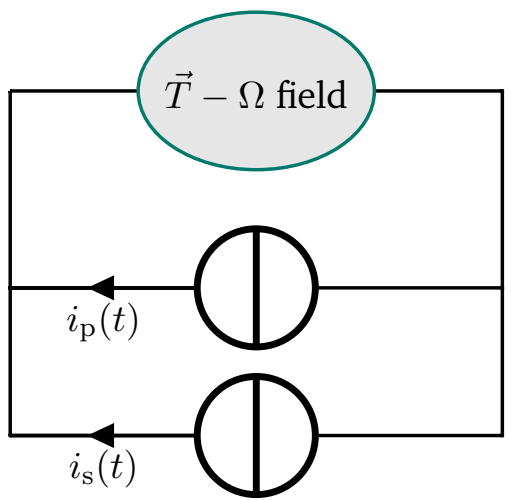

(d) Index 2 circuit with current source $i_{\mathrm{s}}(t)$ and perturbation $i_{\mathrm{p}}(t)$.

Figure 6.2: The considered index 1 and index 2 circuit examples [55].

contain any I $\mu$-cutset and therefore yields and index 1 DAE. In the second example, the element is excited by a current source $i_{\mathrm{S}}(t)$ as depicted in Figure 6.2b. Here, an $\mathrm{I} \mu$-cutset is generated, which results in an index 2 DAE.

Based on the perturbation index concept of Definition 16, we study the sensitivity towards perturbations of the systems by exciting both examples first with the sinusoidal sources

$$
v_{\mathrm{S}}(t)=\sin \left(2 \pi f_{\mathrm{s}} t\right) \quad i_{\mathrm{S}}(t)=\sin \left(2 \pi f_{\mathrm{s}} t\right),
$$

with frequency $f_{\mathrm{s}}=2 \pi$. In the second case of simulations, small but high frequent perturbed signals are added to the sources

$$
\tilde{v}_{\mathrm{s}}(t)=\sin \left(2 \pi f_{\mathrm{s}} t\right)+\varepsilon_{\mathrm{p}} \sin \left(2 \pi f_{\mathrm{p}} t\right) \quad \tilde{i}_{\mathrm{s}}(t)=\sin \left(2 \pi f_{\mathrm{s}} t\right)+\varepsilon_{\mathrm{p}} \sin \left(2 \pi f_{\mathrm{p}} t\right),
$$

with $\varepsilon_{\mathrm{p}}=10^{-4}$ and $f_{\mathrm{p}}=10^{9} f_{\mathrm{s}}$.

Remark 34. Note that, as the considered example is a linear, smooth and uniquely solvable DAE, the differentiation and perturbation index coincide [130], which motivates the sensitivity towards perturbations analysis carried out in the example. 


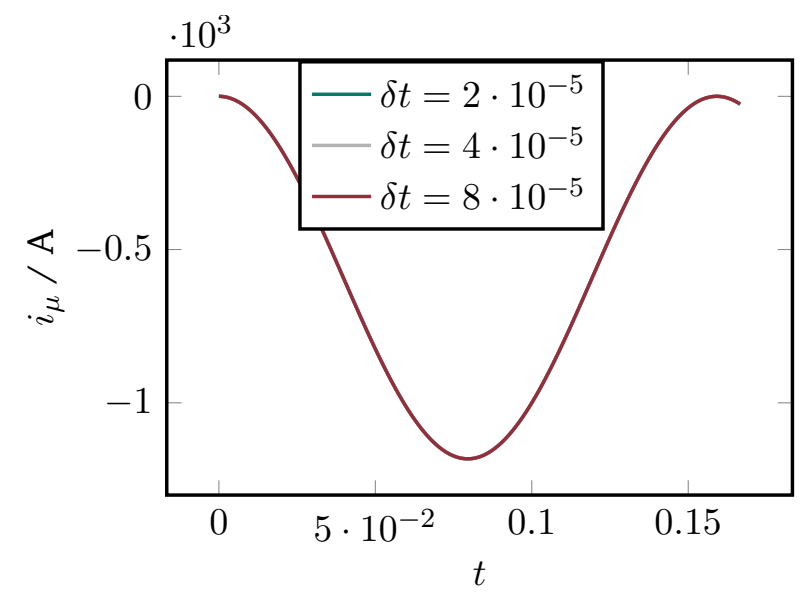

(a) Solution of index 1 circuit with voltage source (Figure 6.2a).

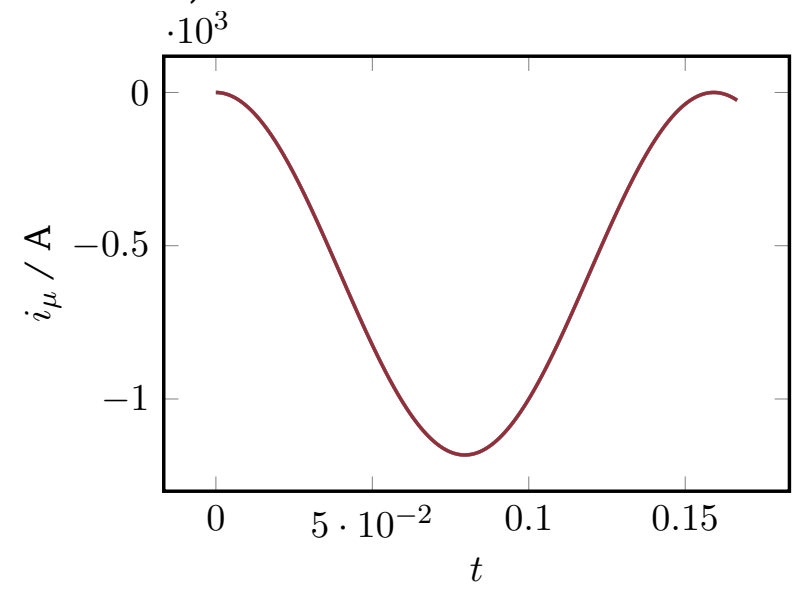

(c) Solution of perturbed index 1 circuit with voltage source (Figure 6.2c).

Figure 6.3: Simulation results for index 1 and index 2 perturbed and non perturbed circuits [55].

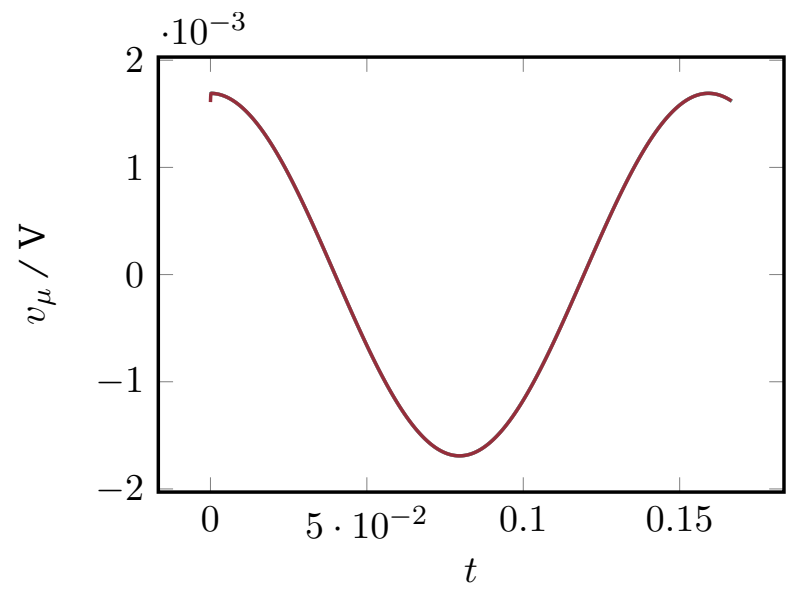

(b) Solution of index 2 circuit with current source (Figure 6.2b).

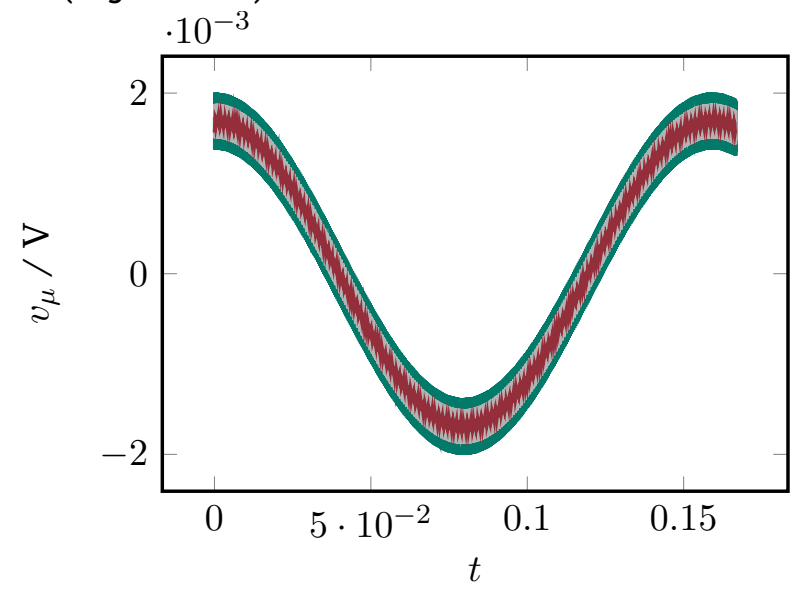

(d) Solution of perturbed index 2 circuit with current source (Figure 6.2d). The legend in (a) holds for all the plots. 
The implicit Euler method is applied on a time interval $\mathcal{I}=\left[\begin{array}{ll}0 & 0.17\end{array}\right]$ with step sizes $\delta t=\left\{8 \cdot 10^{-5}, 4 \cdot 10^{-5}\right.$, $\left.2 \cdot 10^{-5}\right\}$. For the index 1 simulations, consistent zero initial conditions are set at $t_{0}=0$. As it has been shown in the example presented in Section 3.3.2, consistent initialisation of index 2 DAEs is not a trivial issue. Instead of computing the hidden constraints manually and finding such conditions analytically, we exploit the already known property of the implicit Euler scheme (see [129], [132]) that is confirmed in Proposition 19 and set zero initial conditions at time $t=-8 \cdot 10^{-5}$. After at most two implicit Euler steps, the values that are obtained are consistent and therefore, at $t_{0}=0$ all simulations start with a consistent solution.

The simulation results are given in Figure 6.3, where the circuit's lumped quantities that are not given by the corresponding source (voltage or current) are plotted. It can be seen that whereas the small perturbation does not affect the solution in the index 1 DAE, the solution of the index 2 system exhibits oscillations whose amplitude increases the smaller the time-step size of the implicit Euler is set. For time step size $\delta t=2 \cdot 10^{-5}$, the maximal relative error between the perturbed and the non-perturbed simulation result is

$$
\max _{t} \frac{\left|v_{\mu}-\widehat{v}_{\mu}\right|}{\left|v_{\mu}\right|}=3.5511 \cdot 10^{3}
$$

where $v_{\mu}$ and $\widehat{v}_{\mu}$ are the voltage across the inductance-like element of the non-perturbed and perturbed simulations, respectively. This is an expected behaviour for higher index DAEs (see Definition 16) and present only in the circuit with the I $\mu$-cutset. Therefore, the predictions made by the theory (Proposition 7 and Theorem 1) are confirmed.

\subsubsection{Capacitance-like element}

The next simulation consists of an electroquasistatic field approximation in terms of the electric scalar potential (see Section 2.5.2) solved on a cable termination model (see Figure 6.4). As the setting is axisymmetric, only the right-half plane on Figure 6.4 is simulated. The conducting plate $\Omega_{\mathrm{c}}$ and the cable termination $\Omega_{\mathrm{cbl}}$ are modelled through boundary conditions and are therefore also not part of the computational domain. For more details about the dimensions and properties of the model see $[58]^{2}$. The remaining domain is meshed and the PDE is spatially discretised with an in-house code applying the finite element method (linear nodal elements), which yields a semidiscrete system of ODEs with 2708 unknowns. Due to Proposition 11, this system is classified as a capacitance-like element.

Following the results obtained from Theorem 1, a circuit containing a capacitance-like element (and without inductance-like elements) has index 2 if the generalised element is located in a loop together with at least one voltage source ( $\varepsilon \mathrm{V}$-loop). Otherwise, the system has at most index 1. Again we build the simplest circuit cases to obtain both an index 1 and an index 2 DAE. Thus, the element is either coupled to a voltage source $v_{\mathrm{S}}(t)$ (see Figure 6.5b) hereby obtaining a circuit with an $\varepsilon \mathrm{V}$-loop, or with a current source $i_{\mathrm{s}}(t)$ (see Figure $6.5 \mathrm{a}$ ). The sensitivity of the resulting systems towards perturbations is studied analogously to the previous example, as again the system of DAEs is linear and therefore the differential and perturbation index coincide [130]. First the plain source functions (see Figures 6.5a and 6.5b)

$$
v_{\mathrm{s}}(t)=\sin \left(2 \pi f_{\mathrm{s}} t\right) \quad i_{\mathrm{s}}(t)=\sin \left(2 \pi f_{\mathrm{s}} t\right),
$$

\footnotetext{
${ }^{2}$ The code containing the system matrices of the cable termination model can be found in https://github.com/temf/daes_ in_cem
} 


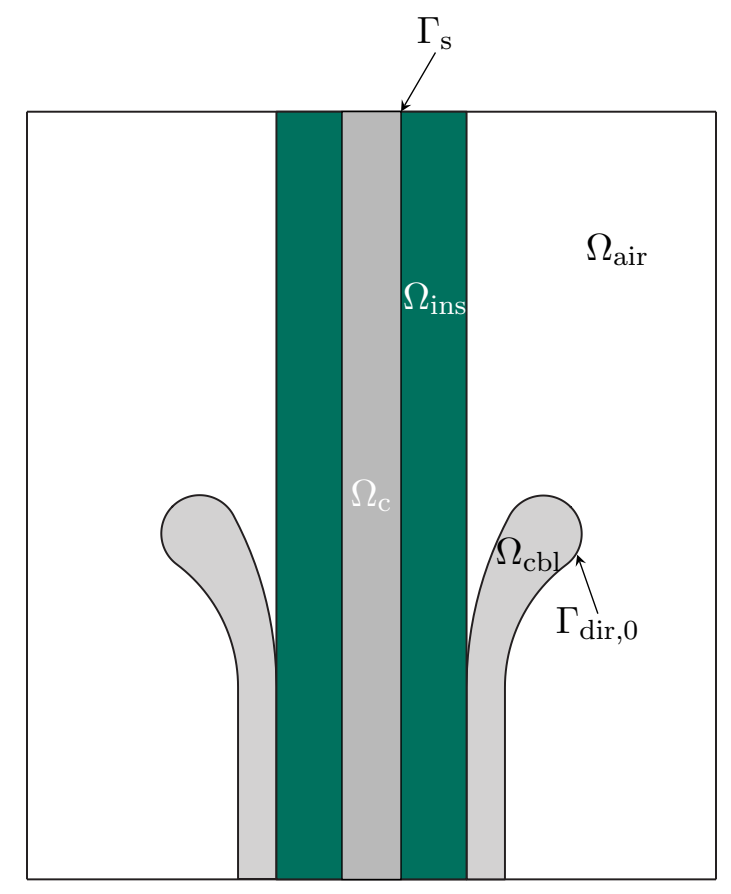

Figure 6.4: Sketch of cable termination model [58].

with frequency $f_{\mathrm{s}}=2 \pi$ are set and then small but high frequent perturbed signals (see Figures $6.5 \mathrm{c}$ and $6.5 \mathrm{~d}$ )

$$
\tilde{v}_{\mathrm{s}}(t)=\sin \left(2 \pi f_{\mathrm{s}} t\right)+\varepsilon_{\mathrm{p}} \sin \left(2 \pi f_{\mathrm{p}} t\right) \quad \tilde{i}_{\mathrm{s}}(t)=\sin \left(2 \pi f_{\mathrm{s}} t\right)+\varepsilon_{\mathrm{p}} \sin \left(2 \pi f_{\mathrm{p}} t\right),
$$

with $\varepsilon_{\mathrm{p}}=10^{-12}$ and $f_{\mathrm{p}}=10^{20} f_{\mathrm{s}}$ are added. The implicit Euler method is applied on the time interval $\mathcal{I}=\left[\begin{array}{ll}0 & 10^{-11}\end{array}\right]$ and with step sizes $\delta t=\left\{10^{-12}, 10^{-13}, 5 \cdot 10^{-14}\right\}$. For the computation of the consistent initial conditions the same approach is taken as in the inductance-like example. The initial conditions for the index 1 simulation are chosen consistently to be zero at $t_{0}=0$. The index 2 case is again handled by starting the simulation at time $t=-2 \cdot 10^{-12}$, such that after at most two implicit Euler iterations at $t_{0}=0$ the solution is consistent.

For a plot of the simulation results see Figure 6.6. The sensitivity towards the small perturbation again affects only one of the two circuit systems, which exhibits qualitatively the same behaviour as the inductance-like element. Here, for time step size $\delta t=5 \cdot 10^{-14}$, the maximal relative error between the perturbed and the non-perturbed simulation result is

$$
\max _{t} \frac{\left|i_{\varepsilon}-\widehat{i}_{\varepsilon}\right|}{\left|i_{\varepsilon}\right|}=9.9615 \cdot 10^{-1}
$$

where $i_{\varepsilon}$ and $\widehat{i}_{\varepsilon}$ are the currents through the capacitance-like element of the non-perturbed and perturbed simulations, respectively. This happens on the circuit with the $\varepsilon$ C-loop, which confirms Proposition 11 and Theorem 1.

\subsubsection{Conclusions}

The section has presented two simulation examples, one of an inductance-like element with a $\vec{T}-\Omega$ magnetoquasistatic system solved on a coil with aluminium core and a capacitance-like element of an electroquasistatic 


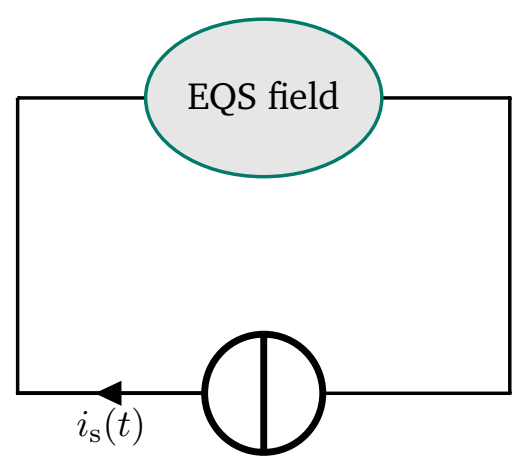

(a) Index 1 circuit with current source $i_{\mathrm{s}}(t)$.

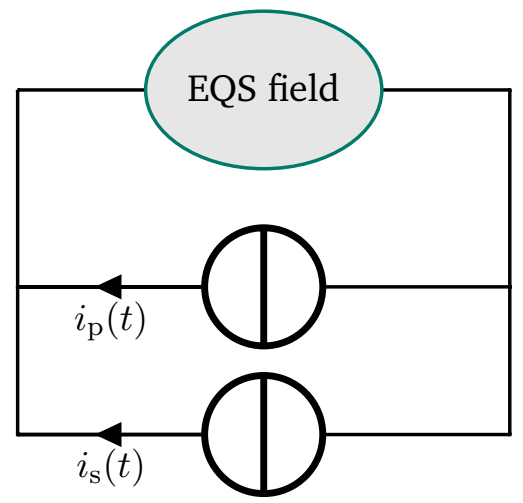

(c) Index 1 circuit with current source $i_{\mathrm{s}}(t)$ and perturbation $i_{\mathrm{p}}(t)$.

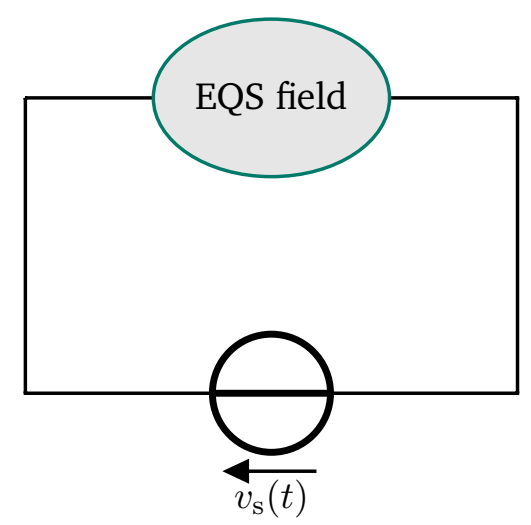

(b) Index 2 circuit with voltage source $v_{\mathrm{s}}(t)$.

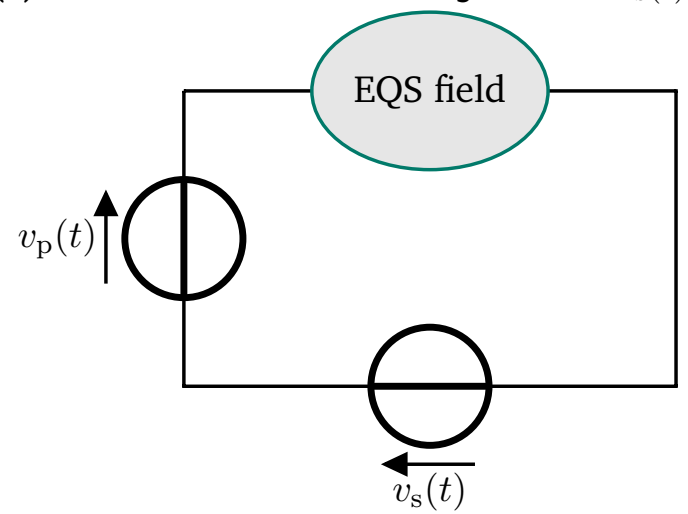

(d) Index 2 circuit with voltage source $v_{\mathrm{s}}(t)$ and perturbation $v_{\mathrm{p}}(t)$.

Figure 6.5: The considered index 1 and index 2 circuit examples.

simulation on a cable termination model. Both have been coupled to circuits that yield index 1 and index 2 DAEs according to the results of Chapter 4. The simulations have backed-up the theoretical results, as in both cases the index 2 circuits built according to Theorem 1 exhibited the typical behaviour of higher index DAEs by showing high sensitivity towards very small high frequent perturbations.

The next section presents simulation results of the waveform relaxation algorithm applied to an index 2 circuit coupled to an eddy current equation.

\subsection{Waveform relaxation for index 2 circuit}

The literature on waveform relaxation typically assumes that the subproblems exchange information only via differential or index-1 variables [28], [29]. It has been shown in [150], [151], that the convergence of the iteration of an index 2 circuit and an inductor model (ODE) depends on the topological properties of the circuit. In the following, the relevance of a (sufficient) convergence criterion is illustrated through one simulation example of a circuit coupled to a transformer described by the eddy current equation (2.20). Here, we focus on the simulation results. More details are presented in [150], [151]. 


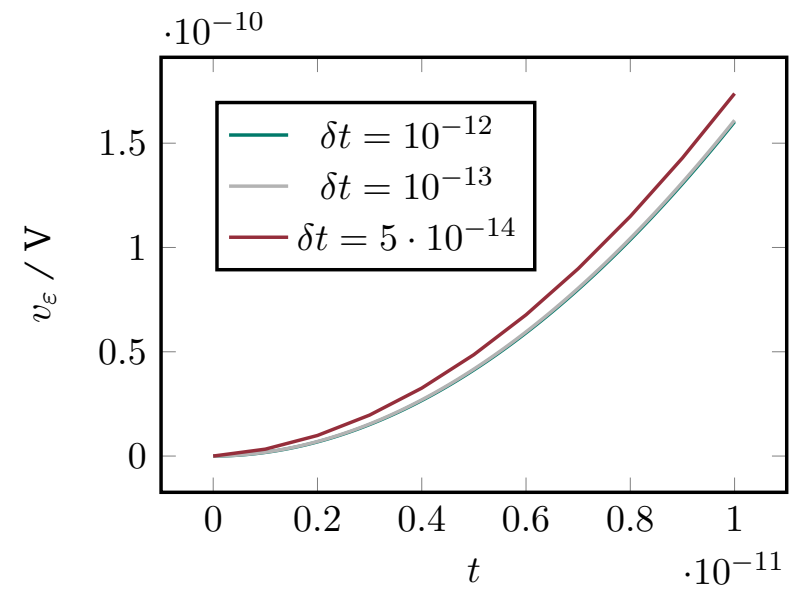

(a) Solution of index 1 circuit with current source (Figure 6.5a).

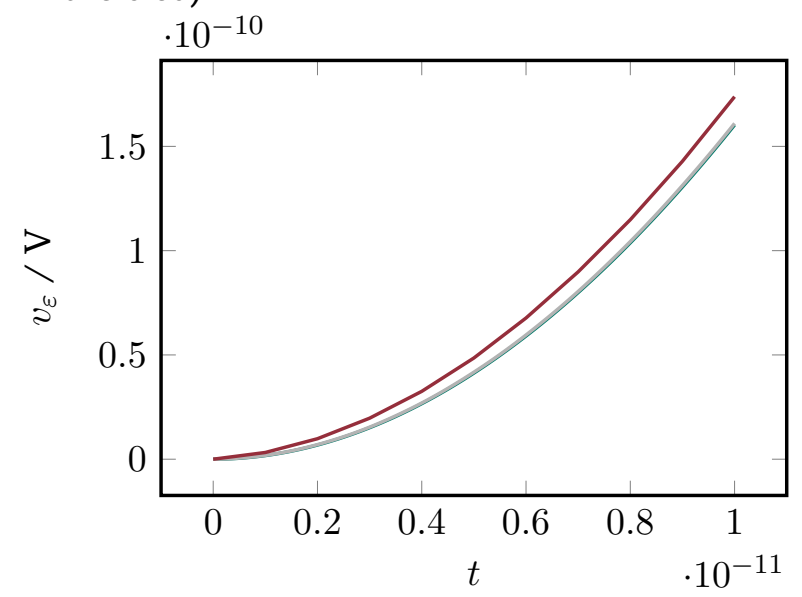

(c) Solution of perturbed index 1 circuit with current source (Figure 6.5c).

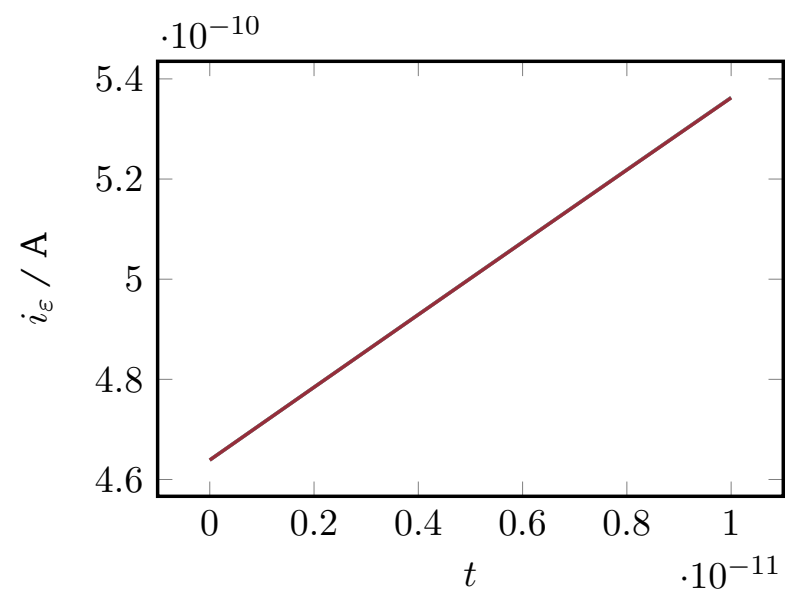

(b) Solution of index 2 circuit with voltage source (Figure $6.5 b)$.

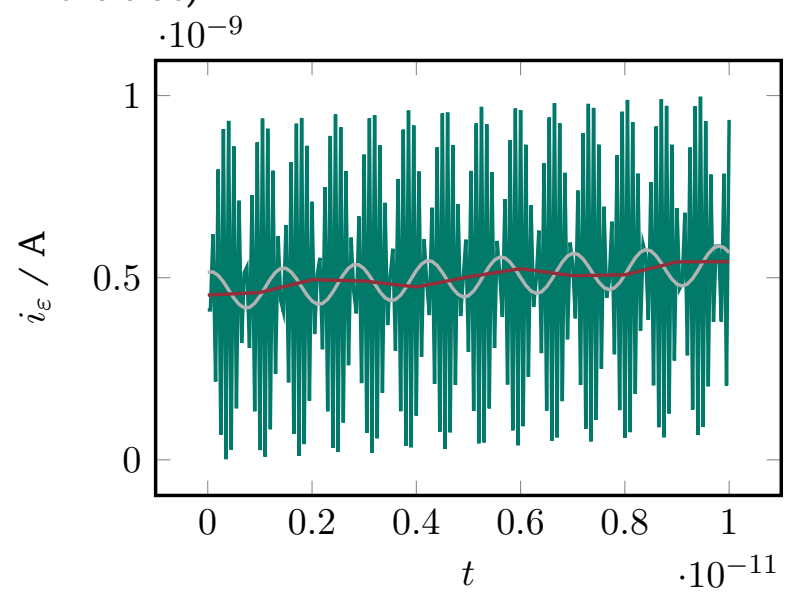

(d) Solution of perturbed index 2 circuit with voltage source (Figure 6.5d).

Figure 6.6: Electroquasistatic simulation results for index 1 and index 2 perturbed and non perturbed circuits. The legend in (a) holds for all the plots. 

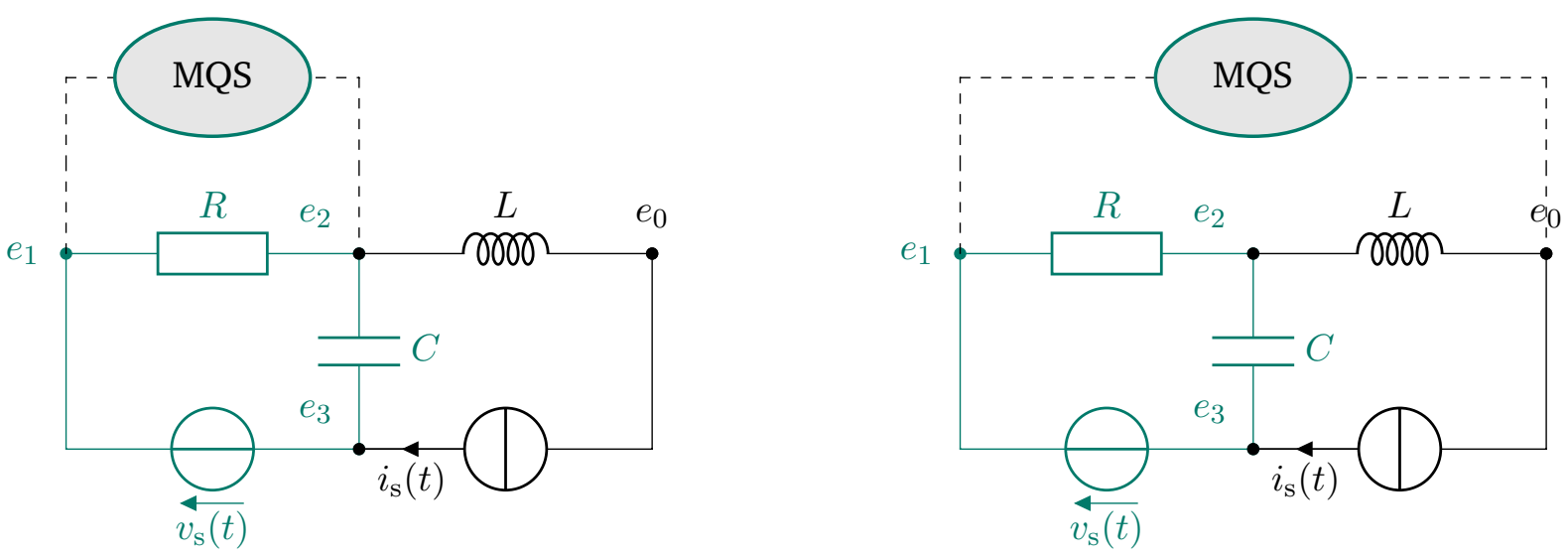

Figure 6.7: Index 2 circuits coupled to magnetoquasistatic field model (dashed) with ground node potential $e_{0}=0$. The CRV components (neglecting the ground node) are highlighted in cyan. Example taken of [151]

Definition 23 (CVR component [150]). Given a graph $G=(V, E)$, we define a CVR component of $G$ as a maximal connected subgraph $G_{\mathrm{CVR}}$ that consists only of capacitances, voltage sources, resistances and their incident nodes.

Let us consider an ODE e.g. describing an inductor, which is coupled to a circuit with the Gauss Seidel scheme

\section{System 1 (element)}

$$
\frac{\mathrm{d}}{\mathrm{d} t} \mathbf{u}^{k+1}+\mathbf{b}\left(\mathbf{u}^{k+1}, t\right)=\mathbf{c}_{1}\left(\mathbf{e}_{\mathrm{c}}^{k}, \mathbf{i}_{\mathrm{c}}^{k}\right),
$$

System 2 (circuit)

$$
\mathbf{A}_{\mathrm{c}}\left(\mathbf{x}^{(k+1)}\right) \frac{\mathrm{d}}{\mathrm{d} t} \mathbf{x}^{(k+1)}+\mathbf{B}_{\mathrm{c}}\left(\mathbf{x}^{(k+1)}, t\right)=\mathbf{c}_{2}\left(\mathbf{u}^{k+1}\right)
$$

where $k$ is the WR iteration counter, $\mathbf{u}$ are the degrees of freedom of the ODE and $\mathbf{x}^{\top}=\left(\mathbf{e}_{\mathrm{c}}^{\top} \mathbf{i}_{\mathrm{c}}^{\top}\right)$ the degrees of freedom of the circuit with $\mathbf{e}_{\mathrm{c}}$ its vector of node potentials and $\mathbf{i}_{\mathrm{c}}$ its vector of currents. Then, the Gauss-Seidel scheme converges if

$$
\sum_{e_{j} \in S_{k}, k \geq 1}\left(\frac{\mathrm{d}}{\mathrm{d} \mathbf{e}_{\mathrm{c}}} \mathbf{c}_{1}\left(\mathbf{e}_{\mathrm{c}}, \mathbf{i}_{\mathrm{c}}\right)\right)_{i j}=0, \forall i,
$$

for $S_{0}, \ldots, S_{n_{\mathrm{CVR}}}$ the $n_{\mathrm{CVR}}+1$ CVR components of the circuit with $S_{0}$ being the ground node [150].

We consider the toy example circuit of Figure 6.7 described by classic modified nodal analysis and with the (arbitrary) parameters for the elements $R=1 \Omega, L=5 \mathrm{H}, C=1 \mathrm{~F}, i_{\mathrm{s}}(t)=\sin (2 t)+5 \sin (20 t)$ and $v_{\mathrm{s}}(t)=$ $\sin (t)+\sin (20 t)$. For the WR scheme, the simulation time window is chosen as $\mathcal{I}=\left[\begin{array}{ll}0 & 0.8\end{array}\right] \mathrm{s}$ and the implicit Euler time step size is $\delta t=10^{-2} \mathrm{~s}$. No windowing is performed, that is, the iterations are applied on the entire simulation time window $\mathcal{I}$. The implementation is performed in Octave.

In contrast to the simulations of [150], the circuit is not coupled to a classic inductance described by an ODE, but to an entire magnetoquasistatic field model (see [151]). Here, the semidiscretised eddy current PDE with circuit coupling equation (see $(2.20),(2.29)$ ) of the transformer in Figure 6.8 is co-simulated with a surrounding circuitry. For simplicity, only the primary coil (light orange in Figure 6.8) is connected to the circuit, which is equivalent to imposing a zero current on the secondary coil. Even though in this setting, the 


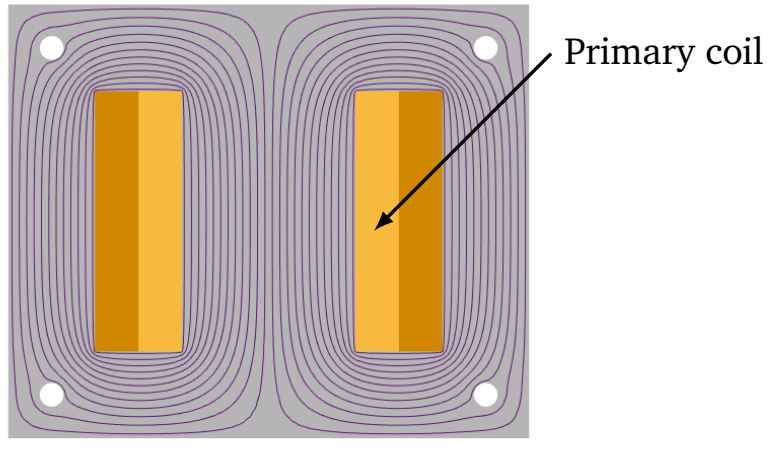

Figure 6.8: Single phase isolation transformer in FEMM (see [162]). ${ }^{3}$

index 2 DAE describing the circuit is coupled to an index 1 DAE instead of an ODE, in [151] it is shown that the theory of [150] still holds.

The information exchange for the eddy current equation is achieved through the coupling equation (2.29) as

$$
\mathbf{X}_{\mathrm{s}}^{\top} \frac{\mathrm{d}}{\mathrm{d} t} \mathbf{a}^{k+1}=\mathbf{c}_{1}\left(\mathbf{e}_{\mathrm{c}}^{k}, \mathbf{i}_{\mathrm{c}}^{k}\right),
$$

where

$$
\mathbf{c}_{1}\left(\mathbf{e}_{\mathrm{c}}^{k}, \mathbf{i}_{\mathrm{c}}^{k}\right)=e_{1}^{k}-e_{2}^{k}
$$

for the first coupling topology (Figure 6.7 left) and

$$
\mathbf{c}_{1}\left(\mathbf{e}_{\mathrm{c}}^{k}, \mathbf{i}_{\mathrm{c}}^{k}\right)=e_{1}^{k}
$$

for the second coupling topology (Figure 6.7 right). Thus, analogously to the ODE case in [150], for node potentials $\mathbf{e}_{\mathrm{c}}=\left[e_{1} e_{2} e_{3}\right]^{\top}$

$$
\sum_{e_{j} \in S_{k}, k \geq 1}\left(\frac{\mathrm{d}}{\mathrm{d} \mathbf{e}_{\mathrm{c}}} \mathbf{c}_{1}\left(\mathbf{e}_{\mathrm{c}}, \mathbf{i}_{\mathrm{c}}\right)\right)_{i j}=1-1=0
$$

in the first coupling approach and thus the WR scheme converges, whereas in the second case

$$
\sum_{e_{j} \in S_{k}, k \geq 1}\left(\frac{\mathrm{d}}{\mathrm{d} \mathbf{e}_{\mathrm{c}}} \mathbf{c}_{1}\left(\mathbf{e}_{\mathrm{c}}, \mathbf{i}_{\mathrm{c}}\right)\right)_{i j}=1 \neq 0
$$

and thus the convergence criterion is not fulfilled.

The simulation results for both approaches are illustrated in Figure 6.9. It can be seen that the WR scheme converges for the first coupling approach, as predicted by theory, whereas the second one diverges. This example demonstrates that the index analysis is important when using involved algorithms such as the waveform relaxation method. For higher index DAEs, slight changes, such as different coupling topologies, can yield divergent iteration schemes even for simple, linear problems. Therefore, when applying the WR algorithm, it is important to have a solid understanding of the structural properties of the underlying subsystems to ensure the iteration scheme behaves as expected.

The next section presents simulation results for the optimised waveform relaxation algorithm of Section 5.1 applied to index 1 systems.

\footnotetext{
${ }^{3}$ The data of the transformer model can be found in http : / /www. femm. info/wiki/MyTransformer.
} 


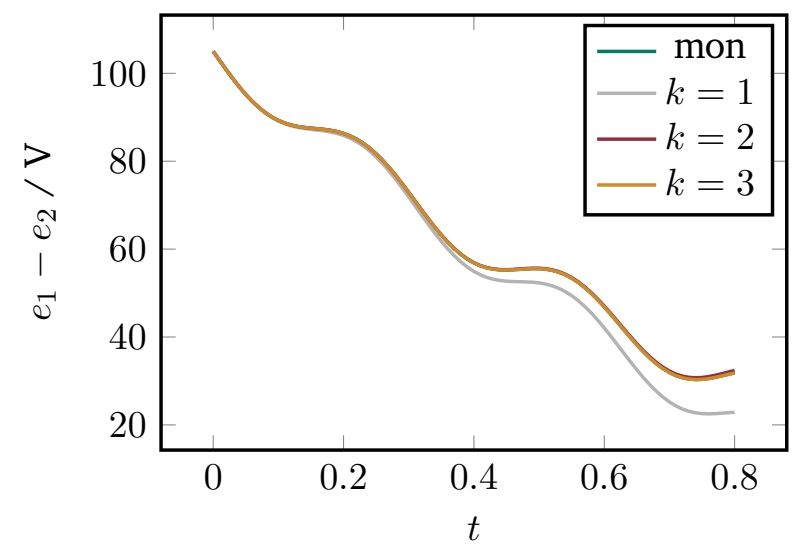

(a) Convergent coupling approach (left setting in Figure 6.7).

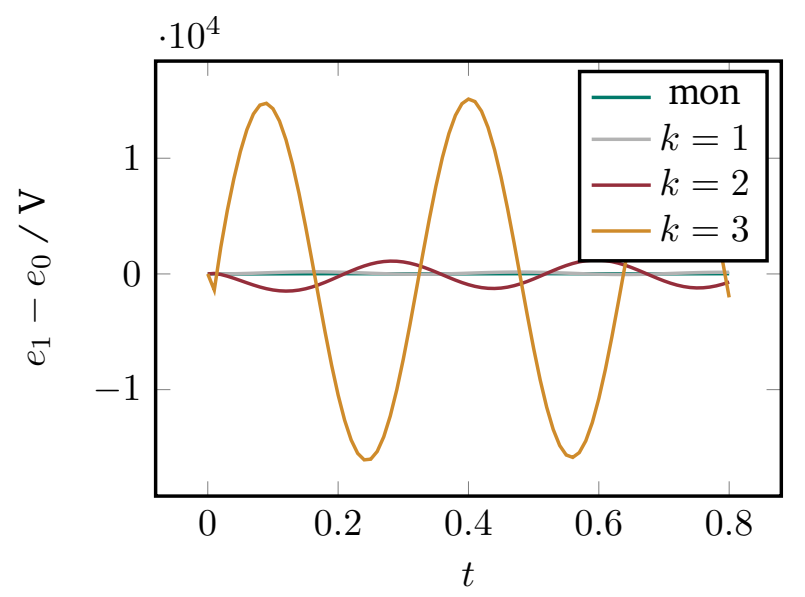

(b) Divergent coupling approach (right setting in Figure 6.7).

Figure 6.9: Voltage across transformer for monolithic solution "mon" and $k$-th WR iteration.

\subsection{Optimised Co-Simulation of Field-Circuit Systems}

In the following, the optimised waveform relaxation algorithm for field-circuit coupled systems presented in Section 5.1 is applied to a numerical example of an accelerator magnet, that is, a resistance-like element (see Section 4.3.4), coupled to a surrounding circuit. The field element's geometry is the single aperture D1 dipole magnet, which is designed for the high luminosity upgrade of the Large Hadron Collider (LHC). For more details about its properties and geometry see [163]. In [8], only the magnethothermal PDE is solved on the D1 dipole magnet. The structure and content of this section follows [56], where the co-simulation algorithm proposed in Section 5.1 is applied and evaluated for the given setting. Later, in [65] the same algorithm is applied to a superconducting quadrupole magnet with, in addition, inter-strand coupling currents.

Let us start by describing how the two subsystems are discretised and solved on their corresponding software in the co-simulation framework.

The computational domain of the magnetothermal PDE is the two dimensional cross-section of the magnet. Furthermore, the symmetry of the domain is exploited and only one quarter of the magnet is simulated (see Figure 6.10a). Homogeneous Dirichlet boundary conditions are set on the outer boundary and homogeneous Neumann BC are set at the symmetry axis (see (2.21)). The rest of the properties such as for example the nonlinear material laws $\left(\nu, \tau_{\text {eq }}\right)$ are given in [163], [164]. For the spatial discretisation of the magnetothermal PDE (5.2) the finite element method is applied with nodal elements of first order for the thermal and second order for the magnetic equations. The model is implemented and solved with COMSOL Multiphysics ${ }^{\circledR}$ [165], which yields a total amount of 9871 degrees of freedom on the magnetothermal side. A backward differentation formula of variable order and maximum step size $\delta t=10^{-3} \mathrm{~s}$ is used for time integration.

The surrounding protection circuit is given in Figure 6.10b. It is simulated with ORCAD PSPICE ${ }^{\circledR}$ [166] and for the time integration the trapezoidal rule is used with maximum step size $\delta t=10^{-6} \mathrm{~s}$. 


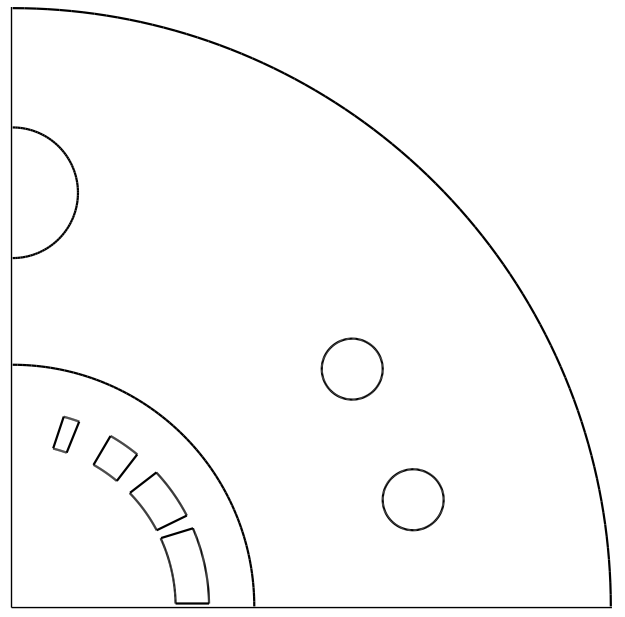

(a) Quarter of the cross section of the single aperture D1 dipole magnet.

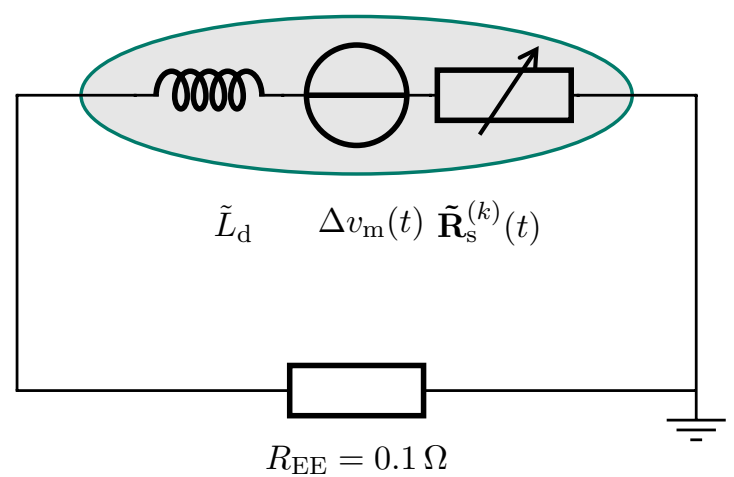

(b) Surrounding protection circuit and lumped model of the optimised transmission condition (ellipse).

Figure 6.10: Superconducting magnet and protection circuit.

Remark 35. Note that the maximum time step size of the field problem $\left(\delta t=10^{-3}\right)$ is much larger than the one of the circuit $\left(\delta t=10^{-6}\right)$. Therefore, in this example the multirate behaviour of the two systems can be exploited within the WR scheme. The magnetothermal problem, which has considerably larger system matrices, is solved with significant lower amount of time steps.

The initial conditions of the coupled problem $\mathbf{a}_{0}$ (magnetic), $\mathbf{T}_{0}$ (thermal) and $\mathbf{x}_{0}$ are computed by first initialising them to zero and then solving the linearised systems separately with a current source excitation that is ramped up from 0 to $5 \cdot 10^{3} \mathrm{~A}$.

For the waveform relaxation, Algorithm 3 is applied on time window $\mathcal{I}=(0 \mathrm{~s}, 0.75 \mathrm{~s}]$ within CERN's inhouse co-simulation framework STEAM [167]. The interval $\mathcal{I}$ is split into 38 subwindows $\mathcal{I}_{j}^{\mathrm{WR}}$ of size $\Delta T_{\mathrm{WR}}=210^{-3} \mathrm{~s}$ and the WR iterations on the $j$-th interval are performed until the stopping criterion

$$
\frac{\int_{T_{j-1}^{\mathrm{WR}}}^{T_{j}^{\mathrm{WR}}}\left|i_{\mathrm{m}}^{(k)}(t)-i_{\mathrm{m}}^{(k-1)}(t)\right| \mathrm{d} t}{\int_{T_{j-1}^{\mathrm{WR}}}^{T_{j}^{\mathrm{WR}}}\left|i_{\mathrm{m}}^{(k)}(t)\right| \mathrm{d} t} \leq \mathrm{tol}
$$

is fulfilled, with $k$ being the WR iteration counter and tol $=10^{-3}$. That is, the iterations are repeated until the difference between two subsequent currents on the magnetothermal system is considered small enough.

\subsubsection{Transmission condition study}

The next step is to study how the waveform relaxation's convergence speed is influenced by the value of the linearised differential inductance in the optimised transmission condition (5.16). To do so, the computed differential inductance, which corresponds to the optimised value derived in Section 5.1.2, is multiplied by a 


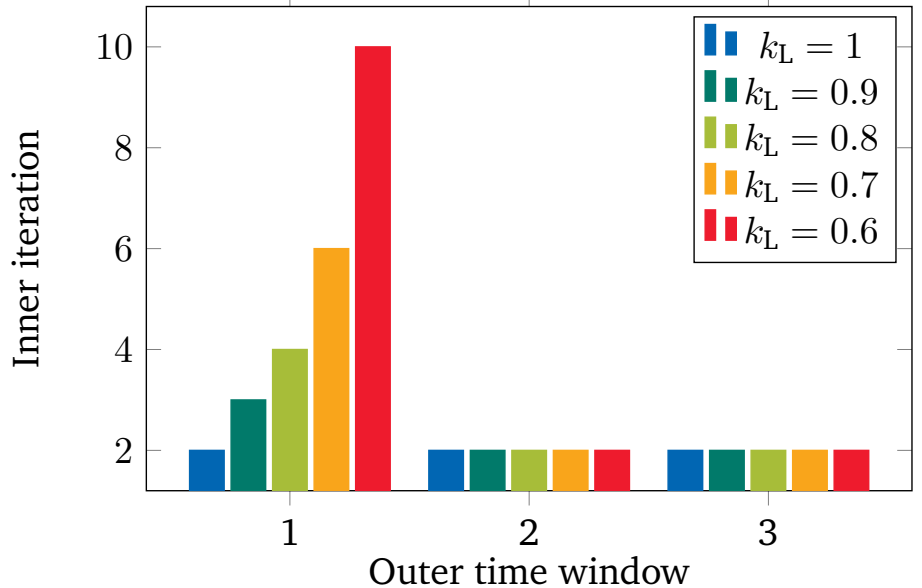

(a) Waveform relaxation iteration numbers for first three windows.

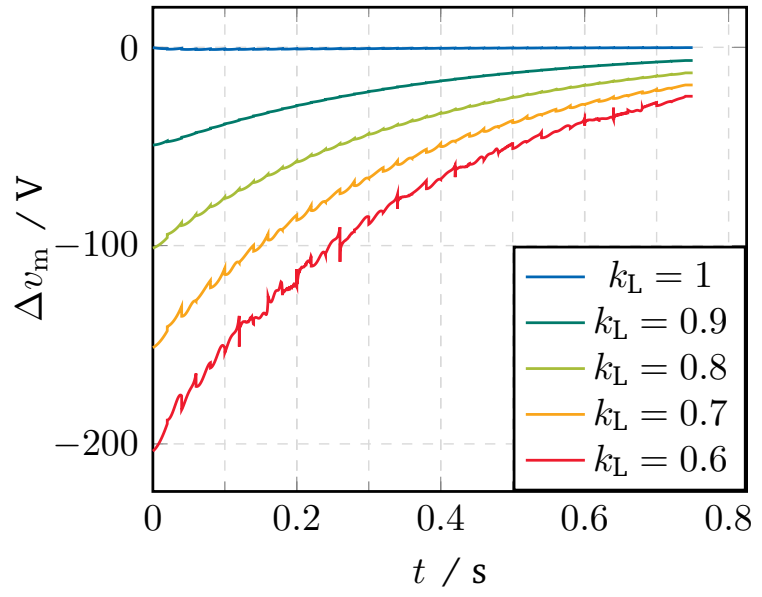

(b) Correction term $\Delta v_{\mathrm{m}}$ between circuit and field solutions.

Figure 6.11: Magnetostatic simulation results.

coefficient $k_{\mathrm{L}}$, that changes its value from 0.5 to 1 . That is, the transmission condition (5.16) is changed to

$$
v_{\mathrm{c}}^{(k+1)}(t)=\tilde{L}_{\mathrm{d}} \frac{\mathrm{d}}{\mathrm{d} t} i_{\mathrm{c}}^{(k+1)}(t)+\Delta v_{\mathrm{m}}^{(k)}(t)
$$

with

$$
\begin{aligned}
\Delta v_{\mathrm{m}}^{(k)}(t) & :=v_{\mathrm{m}}^{(k)}(t)-\tilde{L}_{\mathrm{d}} \frac{\mathrm{d}}{\mathrm{d} t} i_{\mathrm{m}}^{(k)}(t) \\
\tilde{L}_{\mathrm{d}} & :=k_{\mathrm{L}} \mathbf{X}_{\mathrm{s}}^{\top} \mathbf{K}_{\nu, \mathrm{d}}^{-1}\left(\mathbf{a}_{0}\right) \mathbf{X}_{\mathrm{s}} .
\end{aligned}
$$

For $k_{\mathrm{L}}=1$ the optimised transmission condition of Section 5.1.2 is used, and the smaller $k_{\mathrm{L}}$ becomes, the more the information exchange differs from the optimised choice derived in Section 5.1.2. To compute the correction term $\Delta \mathbf{v}_{\mathrm{m}}^{(k)}(t)$ on the circuit side, the waveforms obtained from the magnetothermal solution are linearly interpolated.

The same study is repeated for two different field models. First, a magnetostatic approximation is considered, that is, without the eddy current term, and then the full magnetoquasistatic PDE with nonzero equivalent time constant $\tau_{\text {eq }}$, is used. In both cases the magnetic reluctivity $\nu$ on the iron yoke is nonlinear. The coefficient $k_{\mathrm{L}}$ is varied

$$
k_{\mathrm{L}}=\{1,0.9,0.8,0.7,0.6,0.5\},
$$

hereby reducing the (real) differential inductance at the initial point.

\section{Magnetostatic model}

In the magnetostatic simulation where $\tau_{\text {eq }}=0$ and consequently $\mathbf{K}_{\mathrm{s}}=0$ in (5.2), the iron yoke does not reach saturation within the considered simulation parameters and the reluctivity behaves constantly. Often these simplified models are used as a first test approximation for the simulation of the quench protection systems [168]. 


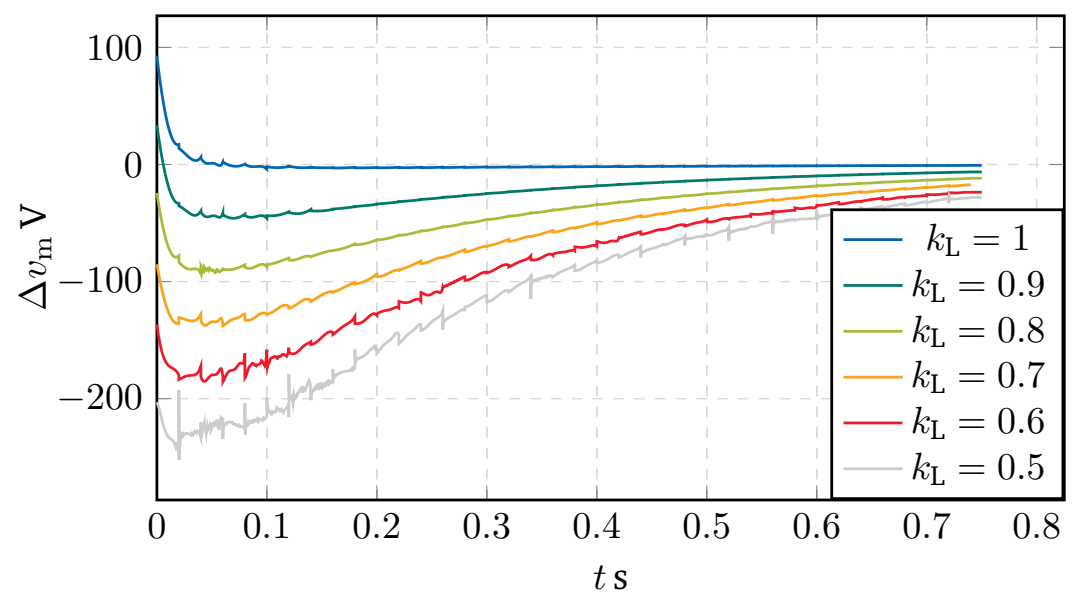

Figure 6.12: Correction term $\Delta v_{\mathrm{m}}$ between circuit and field solutions for eddy current simulation.

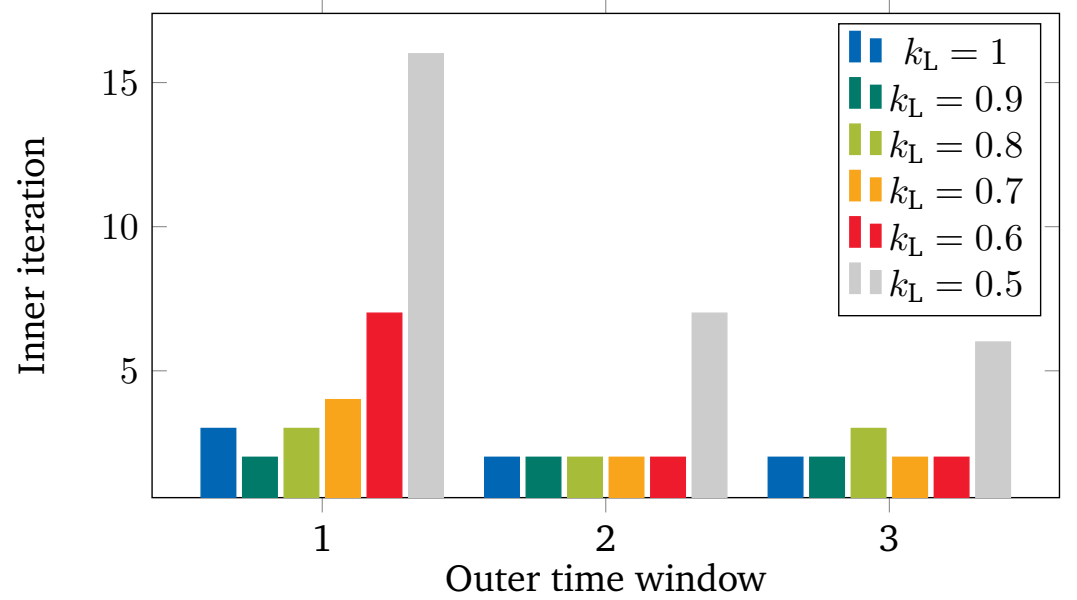

Figure 6.13: Waveform relaxation iteration numbers in the first three windows of the eddy current simulation.

The results of this simulation can be seen in Figure 6.11. Here, the amount of WR iterations required to reach the chosen tolerance are given in Figure 6.11a. Figure 6.11b shows the evolution of the correction voltage over time. As expected, for $k_{\mathrm{L}}=1$ the WR scheme converges immediately in two iterations. As in this setting, the linear static approximation made to compute $L_{\mathrm{d}}$ actually describes the exact solution of the field system, for $k_{\mathrm{L}}=1$ an exact optimal contraction factor $\rho\left(L_{\mathrm{d}}\right)=0$ is obtained. Decreasing $k_{\mathrm{L}}$ worsens the information exchange, as $\tilde{L}_{\mathrm{d}}$ does not describe the exact voltage-to-current relation of the magnetothermal model any more, and thus the amount of iterations required to converge increases. For $k_{\mathrm{L}}=0.5$ the algorithm does not converge.

\section{Magnetoquasistatic model}

In the second simulation, the full magnetothermal nonlinear system (5.2) is used. Again, Figure 6.12 shows the evolution of the correction term $\Delta v_{\mathrm{m}}(t)$ and Figure 6.13 the number of WR iterations required to converge according to criterion (6.1). For this scenario, however, the least amount of iterations are obtained for 


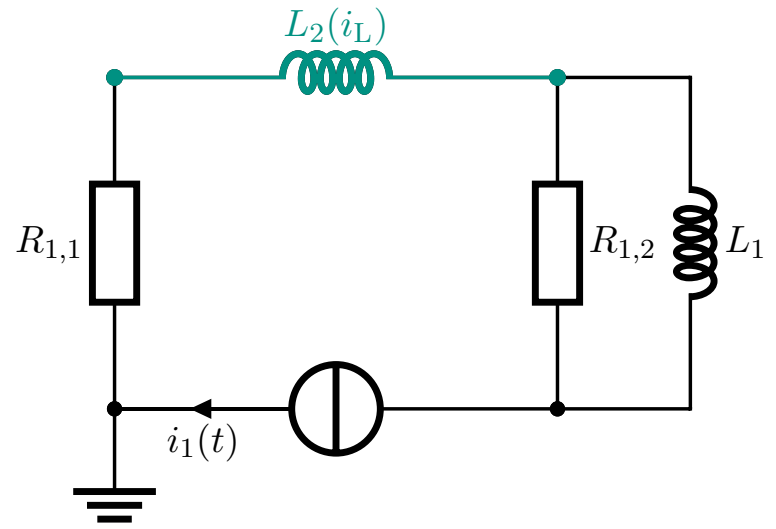

(a) Circuit with nonlinear inductance $L_{2}\left(i_{\mathrm{L}}\right)$ according to the model presented in [169].

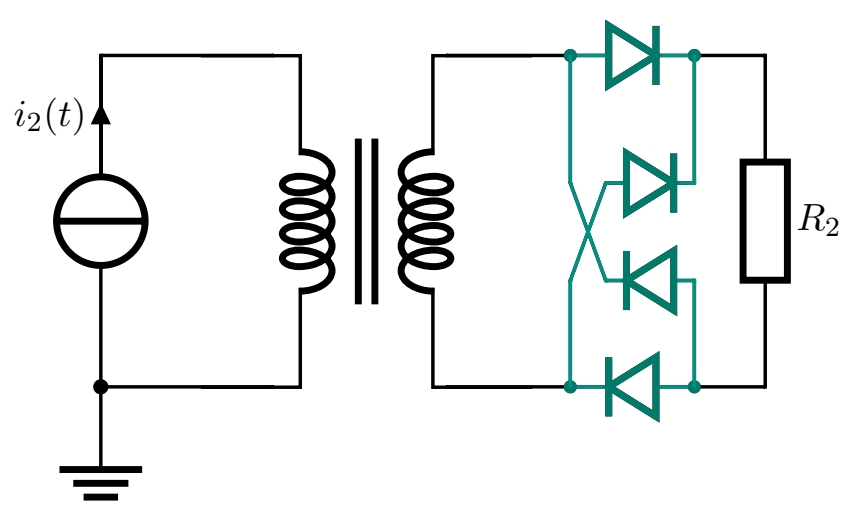

(b) Circuit with nonlinear Shockley diodes as implemented in OCS.

Figure 6.14: Index 2 circuits with LI-cutsets. Nonlinear elements are highlighted in cyan.

$k_{\mathrm{L}}=0.9$, instead of $k_{\mathrm{L}}=1$. This happens due to the losses that dissipate energy in the coil and thus the (time dependent) differential inductance decreases in time. As a consequence, a smaller $\tilde{L}_{\mathrm{d}}$ can yield a better approximation of the voltage-to-current relation of the field system. Note that, therefore, the computed transmission condition is not optimal, but optimised (see Section 5.1.2). This is not only a consequence of the Neumann series approximation of the impedance, but also due to the linearisation of the differential inductance in 6.2. Nevertheless, the amount of WR iterations for $k_{\mathrm{L}}=1$ is significantly lower than e.g. for $k_{\mathrm{L}}=0.5$. In this case, the iteration scheme still converges however takes $k=16$ iterations to do so.

\subsubsection{Conclusions}

This section has presented magnetothermal simulations of the D1 aperture dipole magnet coupled to a surrounding circuitry with the optimised waveform relaxation algorithm presented in Section 5.1. The convergence improvement obtained from the optimised transmission condition has been validated by applying the WR scheme with modifications in the transmission condition. The results show the importance of using suitable transmission conditions, as the total amount of iterations could be decreased from 16 in the worst to 2 in the best case.

The following section deals with Parareal applied to nonlinear index 2 DAEs to further speed up simulation.

\subsection{Parareal for DAEs with Implicit Euler}

To test the behaviour of Parareal when applied to index 2 DAEs with the structure presented in Section 5.2.2, we test the algorithm for two nonlinear circuits with LI-cutsets which yield index 2 DAEs according to Theorem 1. Due to Proposition 19, if the implicit Euler method is applied to nonlinear index 2 DAEs like the ones obtained from flux-charge MNA, consistent solutions are obtained after at most two time steps. Therefore, Parareal is able to converge without requiring a special handling, when applied to those type of systems with 
Index 2 component

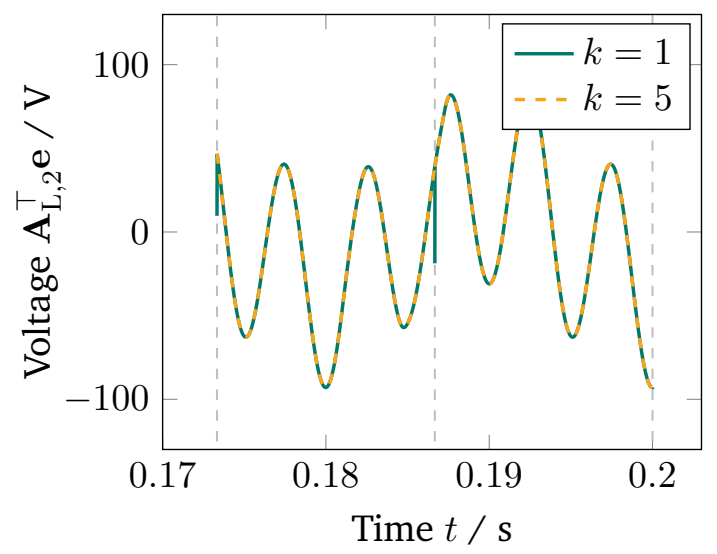

Index 0 component

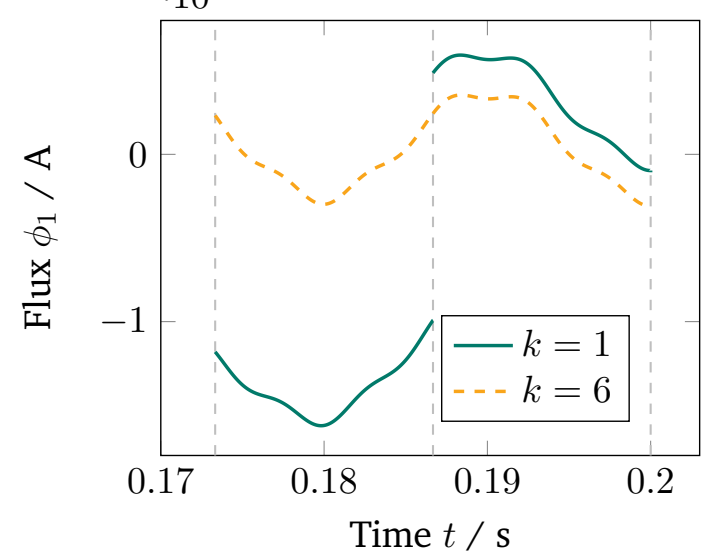

Figure 6.15: First circuit (Figure 6.14a) simulation results at the $k$-th Parareal iteration.

implicit Euler as time integration method. Here, the time integrator itself (Euler) ensures obtaining consistent values and propagating the information of the purely differential components. The circuits are simulated with OCS (Octave Circuit Simulator) ${ }^{4}$, which uses flux-charge MNA to describe their behaviour. To illustrate how the different variables behave during the algorithm, we plot the evolution of both index 2 components as well as the purely differential ones for different Parareal iterations.

The simulation time window $\mathcal{I}=[0,0.2)]$ is divided into $N_{\mathrm{PR}}=15$ subwindows. Both coarse and fine propagators use the implicit Euler however, the first one with one time step per window $(\delta t=0.2 / 15)$, whereas the second one with time step size $\delta t=10^{-5}$.

In the first simulation, the circuit in Figure 6.14a is solved. The linear parameters are chosen as $R_{1,1}=$ $R_{1,2}=10^{-3} \Omega, L_{1}=10^{-4} \mathrm{H}$ and the current source $i_{1}(t)=(100 \sin (100 \pi t)+50 \sin (400 \pi t)) \mathrm{A}$. The nonlinear inductance $L_{2}\left(i_{\mathrm{L}}\right)$ is computed according to the model in [169] with parameter values $L_{\text {nom }}=10^{-3} \mathrm{H}$, $L_{\text {deepsat }}=810^{-4} \mathrm{H}, \sigma=510^{-2}$ and $I_{\mathrm{L}}^{*}=90 \mathrm{~A}$. In Figure 6.15, the evolution on the last two time windows $\mathcal{I}_{14}^{\mathrm{PR}}$ and $\mathcal{I}_{15}^{\mathrm{PR}}$ is shown for the index 2 variable $v_{\mathrm{L}, 1}=\mathbf{A}_{\mathrm{L}, 1}^{\top} \mathbf{e}$, that is, the voltage across the branch of the nonlinear inductance $L_{2}\left(i_{\mathrm{L}}\right)$ and the index 0 variable (purely differential component) $\phi_{2}$, which corresponds to the flux across the linear inductance $L_{1}$. The differential component $\phi_{2}$ shows the typical behaviour of solutions obtained with Parareal. In the first iteration a big jump is visible at the interface which, on the 5th iteration is reduced to a sufficiently continuous solution (relative error of $10^{-5}$ ). The index 2 component $v_{\mathrm{L}, 1}$, however, progresses differently. The starting value in the 1st iteration is inconsistent and it can be observed that the implicit Euler method evolves to the consistent solution which, in this case, even is the exact solution in the sense of the fine propagator. After the 5th iteration, the algorithm has reached a relative $l^{2}$ error of $10^{-5}$ on all the jumps and the solution is sufficiently smooth again.

The second simulation corresponds to the circuit in Figure $6.14 \mathrm{~b}$. The linear resistance is set to $R_{2}=10 \Omega$, the mutual inductance

$$
\mathbf{L}=\left(\begin{array}{cc}
7.0211 & -4.0392 \\
-4.0392 & 2.3247
\end{array}\right)
$$

and the current source is $i_{2}(t)=100 \sin (200 \pi t)$. The reverse currents on the nonlinear Shockley diode models implemented in OCS have $I_{\mathrm{s}}=1.210^{-4}$. Again the evolution of the index 0 and index 2 variables can be

\footnotetext{
${ }^{4}$ https://wiki.octave.org/0cs_package
} 

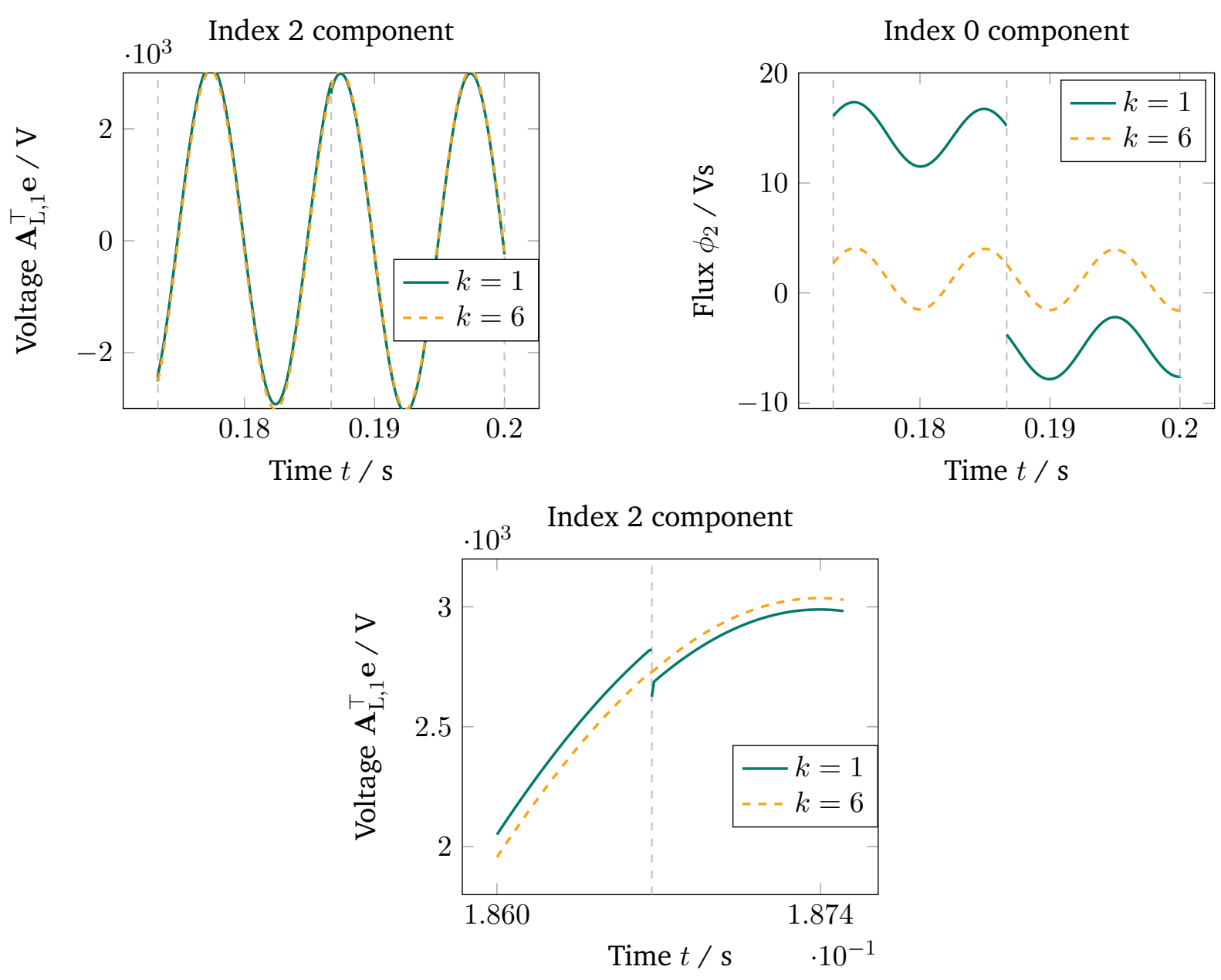

Figure 6.16: Second circuit (Figure 6.14b) simulation results at the $k$-th Parareal iteration. 


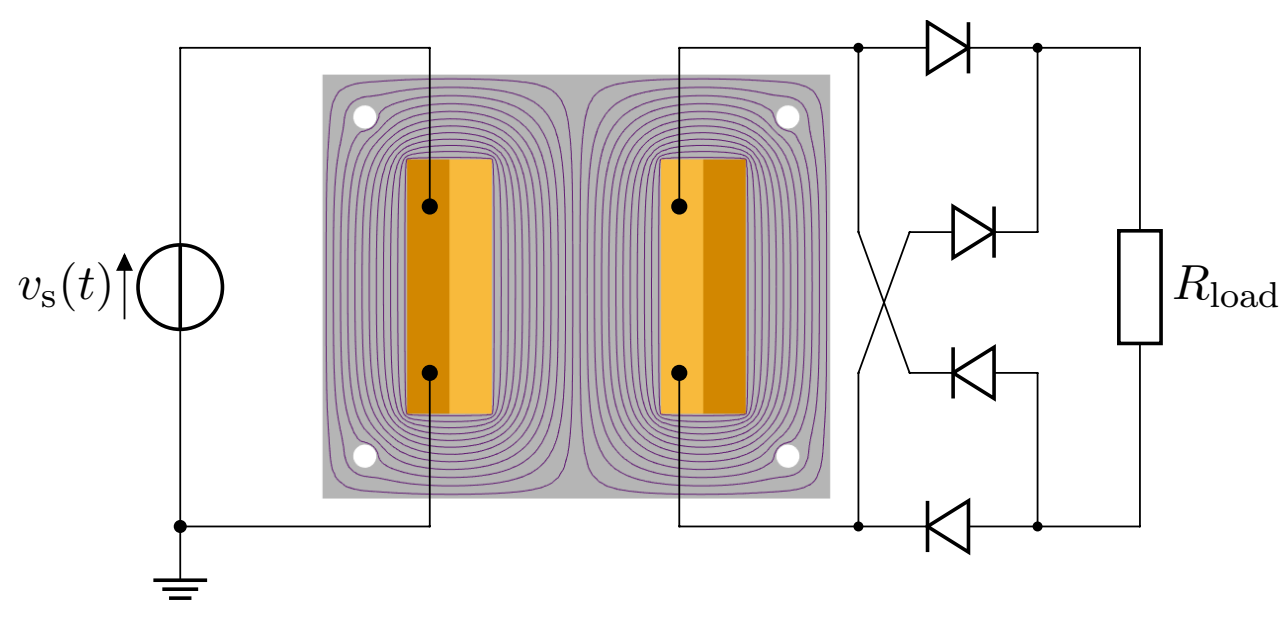

Figure 6.17: Single-phase isolation transformer of model example in FEMM [162] and surrounding rectifier circuit [113, Section 6.3]. Figure taken from [24]. @ [2020] IEEE.

visualised in Figure 6.16. Their behaviour is analogous to the previous example, however, this time Parareal took 6 iterations to reach the relative $l^{2}$ error of $10^{-5}$ on the jumps.

In all the examples, the Parareal algorithm converges to the required tolerance after at most 6 iterations. The results verify that using implicit Euler as time integration method inside the classic Parareal algorithm suffices for these type of nonlinear index 2 DAEs. This allows an easier handling of the Parareal algorithm applied to DAEs arising e.g. from MNA, as no special software is required to compute consistent initial conditions nor further knowledge of the particularities of DAEs systems. Furthermore, the algorithm does not have to be improved or changed.

In the following section the parallelised waveform relaxation algorithm is applied to a field-circuit coupled test example.

\subsection{Parallelised Co-Simulation of Field-Circuit Systems}

We consider the field-circuit coupled example used in [113, Fig. 6.6 (b)] for the numerical tests of the combination of Parareal and waveform relaxation presented in Section 5.3. It consists of a single-phase isolation transformer coupled to a rectifier circuit (see Figure 6.17). Two coupling approaches are applied to the test example. In the first one both the fine and coarse propagators perform a WR scheme. The second approach yields a micro-macro-like algorithm, where the coarse model only solves the circuit part. The example, structure and content of this section is based on [24].

The simulation parameters are as follows. The transformer is a model example of the finite element software $\mathrm{FEMM}^{5}$ with field-independent materials, whose semidiscrete system obtained from the eddy current model yields a linear DAE. The eddy currents are computed with the two dimensional lamination model of [161]. Here, the finite element mass matrix in the field part (5.37) accounting for the eddy currents reads

$$
\mathbf{M}_{i, j}=\int_{\Omega} \frac{1}{12} d^{2} \operatorname{grad} u_{i} \cdot \sigma \operatorname{grad} u_{j} \mathrm{~d} \Omega
$$

\footnotetext{
${ }^{5}$ http : / /www.femm.info/wiki/MyTransformer
} 
where $u_{i}, u_{j}$ are $\mathrm{H}^{1}$-conforming basis (and test) functions (see Section 3.1.2). The circuit's model parameters are given in [113, Section 6.3]. A slight modification is made in the voltage source, which is set to $v_{\mathrm{s}}(t)=$ $220 \sin (2 \pi f t) \mathrm{V}$ with frequency $f=200 \mathrm{~Hz}$. Zero initial conditions are set on all the unknowns.

The simulation time window $\mathcal{I}=[0,0.1)$ is partitioned into $N_{\mathrm{PR}}=N_{\mathrm{WR}}=N$ windows of size $\Delta T$ for both Parareal as well as the waveform relaxation algorithm. Implicit Euler is used for the time integration of the WR algorithm in the coarse and fine propagators. For the coarse solution the time step size is set to $\Delta T$, that is, one time step per window is performed $(\delta t=0.1 / N)$, and the fine solver uses a time step size of $\delta t=5 \cdot 10^{-5}$. The Parareal algorithm is stopped when a relative $l^{2}$ error of $10^{-5}$ is attained on all the jumps.

To evaluate the performance of the algorithm, the effective number of linear system solves (ELSS) is given, which counts how often linear systems are solved in a sequential manner. Here, the solves that are performed in parallel and communication costs are neglected.

\subsubsection{Parallelised waveform relaxation}

The first algorithm that is considered is the simple coupling approach of Parareal and WR explained in Section 5.3.1. Here, Parareal is applied with its classic version as given in Algorithm 4 and the propagators are implemented as follows.

- $\mathcal{G}\left(T_{i}, T_{i-1}, \mathbf{X}_{i-1}^{k+1}\right)$ performs the waveform relaxation algorithm with optimised transmission condition (Algorithm 3). The first system is chosen to be the magnetoquasistatic field with lamination eddy currents (5.37) and voltage transmission condition for the $k$-th iteration

$$
\mathbf{v}_{\mathrm{m}}^{k+1}=\mathbf{v}_{\mathrm{c}}^{k} .
$$

The second system corresponds to the circuit (5.38a)-(5.38b) with the optimised transmission condition of Section 5.1.2 for the $k$-th iteration

$$
\mathbf{v}_{\mathrm{c}}^{k+1}=\mathbf{L i}_{\mathrm{c}}^{k+1}-\mathbf{L i}_{\mathrm{m}}^{k+1}+\mathbf{v}_{\mathrm{m}}^{k+1} .
$$

Only a fixed amount of WR iterations $k_{\mathrm{c}}=1.5$ are carried out. This corresponds to one iteration where both systems are solved and then performing 'half' an iteration more, where only the field system is simulated.

- $\mathcal{F}\left(T_{i}, T_{i-1}, \mathbf{X}_{i-1}^{k+1}\right)$ applies the WR method with the same setting as the coarse propagator, however it executes the iterations until a relative $l^{2}$ error of $10^{-8}$ between two subsequent WR solutions of the coupling variables is reached.

This methodology is denoted by the acronym 'PRWR'.

\subsubsection{Field-circuit parallelised waveform relaxation}

For the second algorithm we use the micro-macro-like approach of Section 5.3.1. Here, the Parareal algorithm requires the extra matching update and thus follows Algorithm 5. The propagators are chosen as follows.

- $\mathcal{G}\left(T_{i}, T_{i-1}, \mathbf{X}_{i-1}^{k+1}\right)$ solves only the circuit system (5.38) (see Figure 6.18). 


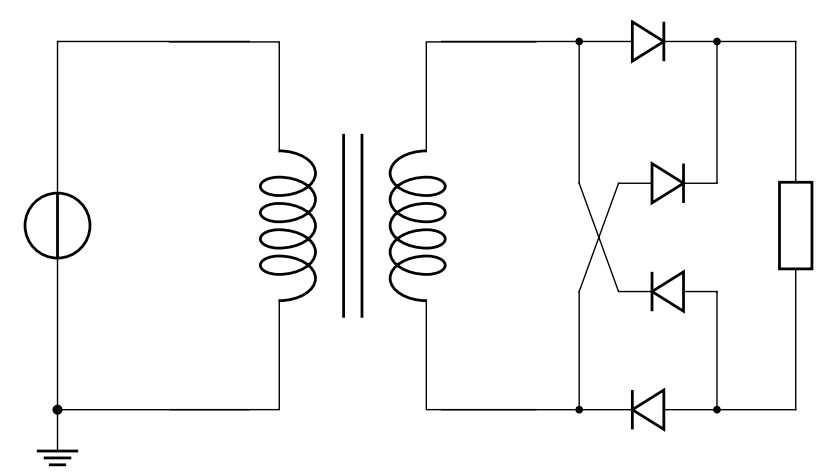

Figure 6.18: Field-Circuit parallelised waveform relaxation coarse system model [24]. ( ) [2020] IEEE.

- $\mathcal{F}\left(T_{i}, T_{i-1}, \mathbf{X}_{i-1}^{k+1}\right)$ is chosen the same as in 'PRWR' and thus WR is iterated to the relative error of $10^{-8}$. Notice that this algorithm requires the definition of a prolongation and restriction operation. This is performed as explained in Section 5.3.1. The algorithm is denoted as 'FCPRWR'.

\subsubsection{Simulation results}

The effective number of linear system solves for sequential WR, PRWR and FCPRWR can be seen in Fig. 6.19. Even though the first algorithm (PRWR) already significantly decreases the ELSS when comparing with the sequential simulation, it can be observed that the algorithm is slowed down by the sequential computations of the coarse solver already for $N=101$. However, the micro-macro-like algorithm benefits from the fact that on the coarse side no FE systems are solved and thus the ELSS for the field part is not affected by larger number of windows $N$.

Note that, the size of the systems on circuit side is significantly smaller than the one of the field, which results of a finite element discretisation of a distributed model. In the present example the field system has 716 degrees of freedom, whereas the circuit only consists of 9 . For real world examples this differences can increase even more, as finite element matrices easily can yield several hundreds of thousands of unknowns. On the second algorithm (FCPRWR), the field system is not solved on the sequential, coarse level, which potentially is beneficial, especially for large space discretisation matrices and would also reduce communication costs (if considered).

\section{Eddy currents without lamination}

The micro-macro-like algorithm has also been applied to the same transformer model with classic eddy currents. For the two dimensional setting, the mass matrix of (3.7) is

$$
\mathbf{M}_{i, j}=\int_{\Omega} u_{i} \sigma u_{j} \mathrm{~d} \Omega
$$

However, in this case, Parareal converges after the $N$-th iteration, which in practice does not give any speed up compared to the sequential simulation. This confirms the foreseen problem Remark 33 that for magnetoquasistatic systems with large eddy current effects, the neglection of the skin effect on the prolongation operation can lead to a too simplified coarse model, which does not describe accurately enough the spatial behaviour of the field. 

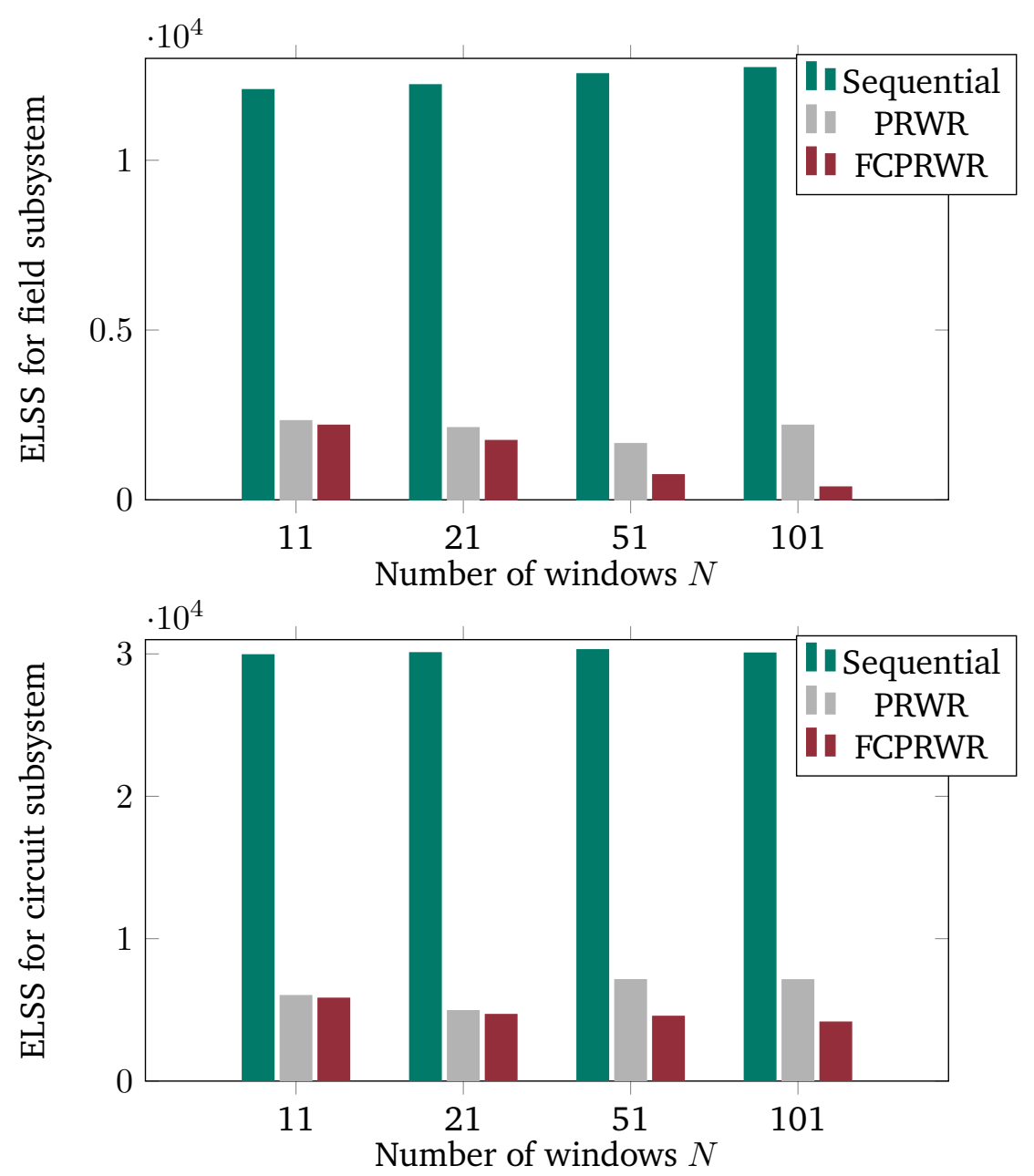

Figure 6.19: Effective number of linear system solves for field-circuit coupled systems with lamination model [24]. Comparison for Sequential waveform relaxation, PRWR and FCPRWR algorithms.

\subsubsection{Conclusion}

The section has presented two numerical tests of a single-phase isolation transformer with lamination eddy currents coupled to a surrounding circuitry simulated with Parareal and waveform relaxation. They correspond to the two algorithms proposed in Section 5.3.1, whose performances have been compared to the sequential simulation. The results have confirmed that both algorithms are able to reduce the computational cost of the co-simulation of field-circuit coupled systems. Furthermore, the micro-macro-like algorithms benefits from the reduced coarse system. For systems with large eddy currents, however, the magnetostatic approximation of the coarse model was not accurate enough and the algorithm has not yield a speed-up. Two possible improvement paths are left for future work. On one hand, more evolved lumped models to better describe the voltage-to-current relation of the field on coarse circuit simplification can be studied. On the other hand, a better prolongation operator that is able to capture the skin effect of the magnetic field is of high importance. 


\section{Summary and Outlook}

This work has dealt with the mathematical analysis and simulation of refined models in circuits. It has focused on the simulation of electromagnetic fields in devices and their surrounding circuitry with the special motivation of quench propagation in accelerator circuits. However, the analysis and idea behind the simulation techniques can be transferred to other type of coupled systems (see [153]).

The structural analysis of the coupled system has been performed in Chapter 4. For that, three generalised circuit elements have been defined that allow the classification of the refined models. Analogous to the classic (differential) DAE index analysis of the modified nodal analysis, a theorem has been derived that formulates the index of the entire coupled system by means of topological properties of the underlying network. In the last part of the chapter, four refined models arising from different formulations, models and approximations to Maxwell's equations have been classified. This has allowed confirming already known results, such that the classic magnetoquasistatic $\vec{A}^{*}$ formulated PDE behaves from the index point of view as an inductance. Furthermore, new index results for other type of field equations in circuits have been obtained. In particular, the $\vec{T}-\Omega$ formulated magnetoquasistatic PDE, the $\vec{A}^{*}$ formulated eddy current homogenisation model for inter-filament coupling currents in superconducting coils and the electroquasistatic system of equations have been classified.

Iterative algorithms for time domain simulations have been studied in Chapter 5 . The co-simulation of magnetoquasistatic field approximations and circuits has been investigated by means of optimised Schwarz methods. With the motivation of the quench simulation in superconducting magnets, the co-simulation of the magnetothermal eddy current model arising from inter-filament coupling currents inside electric networks has been analysed. Here, the transmission condition (information exchange) between the magnetothermal system and the circuit inside the waveform relaxation algorithm has been optimised. Hereby the amount of iterations in the WR scheme that are required to reach the needed tolerance are significantly reduced (from 16 to 2 ). To further speed up simulation time, a second iterative method called Parareal has been studied. The parallelin-time algorithm has first been investigated in the context of higher index DAEs. A generalisation of the algorithm has been presented to be consistently applied to DAEs. For systems with a special structure, such as the ones obtained from flux-charge MNA, it has been shown that the classic Parareal method can be applied if the implicit Euler is used as the time integrator. In the last part, both methods, i.e. waveform relaxation and Parareal, have been combined into one algorithm. Two different coupling strategies for the two methods have been proposed. In the second approach, which is set up for eddy current systems coupled to circuits, a micro-macro-like Parareal algorithm is obtained. Its idea, based on Schur-complements, however, can be expanded to other type of coupled systems.

Chapter 6 has presented several numerical studies to underline the theoretical results obtained previously. First, two different field models are coupled to two different circuits yielding index 1 and index 2 DAEs according to Theorem 1 of Chapter 4. The sensitivity towards perturbations of the index 2 simulations has confirmed the predictions obtained from theory. So as to test the optimised waveform relaxation algorithm, the co-simulation of a magnetothermal model of an accelerator magnet and its surrounding circuitry has been 
carried out. The waveform relaxaion algorithm has been applied with the optimised transmission condition derived in Chaper 5, as well as with modifications of it. The numerical tests have shown the importance of choosing an appropriate information exchange between the subsystems, as the number of iterations to reach the required tolerance could be considerably reduced (2 instead of 16 iterations). To test the Parareal algorithm together with the implicit Euler method as time integrator on nonlinear index 2 systems, the method is applied to two different circuits modelled with flux-charge MNA. The evolution of the solution for index 0 and index 2 variables through the Parareal algorithm has been shown. In the end, two combinations of Parareal and the waveform relaxation algorithm have been applied to a transformer coupled to a circuit. Both algorithms have been able to considerably reduce computational cost for the eddy current model with lamination. In the best case, the effective number of linear systems solves of the field's finite element matrices could be reduced by a factor of 33 .

\subsection{Outlook}

The theoretical and numerical results presented in this work raise new questions to be answered in future work.

The optimised transmission condition in the waveform relaxation algorithm for accelerator magnets in circuits has been approximated by means of a Neumann series. This leaves the possibility of adding more terms of the series to potentially further speed up the iteration convergence. Thus, testing this on numerical examples that require more WR iterations to reach the required tolerance is something that can is left for a continuation of this work. Furthermore, other series approximations could be investigated for alternative transmission conditions.

The next step to take regarding Parareal and DAEs is to consider systems with nonlinear index 2 components, or even higher index, that are not covered by the theoretical results obtained for the implicit Euler method as time integrator. Here, the modified Parareal algorithm for DAEs presented in Section 5.2 should be tested and compared to the classic algorithm.

An improved micro-macro like combination of Parareal and optimised waveform relaxation in field-circuit coupled systems is still to be mathematically analysed to ensure the algorithm can also be applied to field systems with strong eddy current effects. Here, more evolved lumped models can improve the voltage-tocurrent relation of the coarse system to better mimic the behaviour of the fine system. This could for example be obtained by already existing equivalent circuits or by considering more terms of the Neumann series of the optimised Schwarz transmission condition. Another, probably more prominent, source of error is the prolongation operator that is not able to capture the skin effect (in space). Thus, studying improved prolongation operators is another topic for study that is left for future research. 


\section{Bibliography}

[1] A. Bartel and R. Pulch, "A concept for classification of partial differential algebraic equations in nanoelectronics", in Progress in Industrial Mathematics at ECMI 2006, L. L. Bonilla, M. Moscoso, G. Platero, and J. M. Vega, Eds., ser. Mathematics in Industry, vol. 12, Berlin: Springer, Dec. 2007.

[2] I. A. Tsukerman, A. Konrad, G. Meunier, and J. C. Sabonnadière, "Coupled field-circuit problems: Trends and accomplishments", IEEE Trans. Magn., vol. 29, no. 2, pp. 1701-1704, Mar. 1993.

[3] S. Schöps, H. De Gersem, and A. Bartel, "A cosimulation framework for multirate time-integration of field/circuit coupled problems”, IEEE Trans. Magn., vol. 46, no. 8, pp. 3233-3236, Jul. 2010.

[4] P. Ferracin, G. Ambrosio, M. Anerella, et al., "The hl-lhc low- $\beta$ quadrupole magnet mqxf: From short models to long prototypes", IEEE Transactions on Applied Superconductivity, vol. 29, no. 5, pp. 1-9, Aug. 2019.

[5] L. Bortot, B. Auchmann, I. Cortes Garcia, et al., "STEAM: A hierarchical co-simulation framework for superconducting accelerator magnet circuits", IEEE Trans. Appl. Super., vol. 28, no. 3, Apr. 2018.

[6] K. Dahlerup-Petersen, R. Denz, J. L. Gomez-Costa, et al., "The protection system for the superconducting elements of the large hadron collider at CERN", in Proceedings of the 1999 Particle Accelerator Conference (Cat. No.99CH36366), vol. 5, 1999, pp. 3200-3202.

[7] E. Ravaioli, "CLIQ - a new quench protection technology for superconducting magnets", PhD thesis, University of Twente, 2015.

[8] L. Bortot, M. Maciejewski, M. Prioli, et al., "Simulation of electro-thermal transients in superconducting accelerator magnets with COMSOL multiphysics", in Proceedings of the European COMSOL Conference 2016, Munich, Oct. 2016.

[9] M. Maciejewski, P. Bayrasy, K. Wolf, et al., "Coupling of magnetothermal and mechanical superconducting magnet models by means of mesh-based interpolation", IEEE Trans. Appl. Super., vol. 28, no. 3, Apr. 2018.

[10] A. Y. Hannalla and D. C. MacDonald, "Numerical analysis of transient field problems in electrical machines", Proceedings of the Institution of Electrical Engineers, vol. 123, no. 9, pp. 893-898, Sep. 1976.

[11] P. G. Potter and G. K. Cambrell, "A combined finite element and loop analysis for nonlinearly interacting magnetic fields and circuits", IEEE Trans. Magn., vol. 19, no. 6, pp. 2352-2355, Nov. 1983.

[12] J. G. Rollins and J. Choma, "Mixed-mode PISCES-SPICE coupled circuit and device solver", vol. 7, no. 8, pp. 862-867, Aug. 1988.

[13] K. Mayaram and D. O. Pederson, "Coupling algorithms for mixed-level circuit and device simulation", vol. 11, no. 8, pp. 1003-1012, Aug. 1992.

[14] K.-T. Grasser and S. Selberherr, "Mixed-mode device simulation", vol. 31, no. 11, pp. 873-881, 2000. 
[15] M. Günther, "A joint DAE/PDE model for interconnected electrical networks", Math. Model. Syst., vol. 1, no. 1, pp. 000-111, 2000.

[16] M. C. Costa, S. I. Nabeta, and J. R. Cardoso, "Modified nodal analysis applied to electric circuits coupled with FEM in the simulation of a universal motor", IEEE Trans. Magn., vol. 36, no. 4, pp. 14311434, Jul. 2000.

[17] M. Günther and U. Feldmann, "The DAE-index in electric circuit simulation", Math. Comput. Simulat., vol. 39, pp. 573-582, Nov. 1995.

[18] D. Estévez Schwarz and C. Tischendorf, "Structural analysis of electric circuits and consequences for MNA”, Int. J. Circ. Theor. Appl., vol. 28, no. 2, pp. 131-162, 2000.

[19] A. Nicolet and F. Delincé, "Implicit Runge-Kutta methods for transient magnetic field computation", IEEE Trans. Magn., vol. 32, no. 3, pp. 1405-1408, May 1996.

[20] S. Baumanns, M. Clemens, and S. Schöps, "Structural aspects of regularized full Maxwell electrodynamic potential formulations using FIT", in Proceedings of 2013 URSI International Symposium on Electromagnetic Theory (EMTS), G. Manara, Ed., IEEE, May 2013, pp. 1007-1010.

[21] I. A. Tsukerman, "Finite element differential-algebraic systems for eddy current problems", Numer. Algorithm., vol. 31, no. 1, pp. 319-335, Dec. 2002.

[22] A. Bartel, S. Baumanns, and S. Schöps, "Structural analysis of electrical circuits including magnetoquasistatic devices”, APNUM, vol. 61, pp. 1257-1270, Sep. 2011.

[23] G. Alì, A. Bartel, M. Günther, and C. Tischendorf, "Elliptic partial differential-algebraic multiphysics models in electrical network design", M3AS, vol. 13, no. 9, pp. 1261-1278, 2003.

[24] I. Cortes Garcia, S. Schöps, C. Strohm, and C. Tischendorf, "Generalized circuit elements", 2019. arXiv: 1912.05199, Submitted.

[25] E. Lelarasmee, A. E. Ruehli, and A. L. Sangiovanni-Vincentelli, "The waveform relaxation method for time-domain analysis of large scale integrated circuits", IEEE Trans. Comput. Aided. Des. Integrated Circ. Syst., vol. 1, no. 3, pp. 131-145, 1982.

[26] K. Burrage, Parallel and sequential methods for ordinary differential equations. Oxford: Oxford University Press, 1995.

[27] Z. Jackiewicz and M. Kwapisz, "Convergence of waveform relaxation methods for differential-algebraic systems”, SIAM J. Numer. Anal., vol. 33, no. 6, pp. 2303-2317, Dec. 1996.

[28] M. Arnold and M. Günther, "Preconditioned dynamic iteration for coupled differential-algebraic systems", BIT, vol. 41, no. 1, pp. 1-25, 2001.

[29] A. Bartel, M. Brunk, M. Günther, and S. Schöps, "Dynamic iteration for coupled problems of electric circuits and distributed devices", SIAM J. Sci. Comput., vol. 35, no. 2, B315-B335, Mar. 2013.

[30] A. Bartel, M. Brunk, and S. Schöps, "On the convergence rate of dynamic iteration for coupled problems with multiple subsystems", J. Comput. Appl. Math., vol. 262, pp. 14-24, May 2014.

[31] G. Bedrosian, "A new method for coupling finite element field solutions with external circuits and kinematics", IEEE Trans. Magn., vol. 29, no. 2, pp. 1664-1668, 1993.

[32] P. Zhou, D. Lin, W. N. Fu, B. Ionescu, and Z. J. Cendes, "A general co-simulation approach for coupled field-circuit problems”, IEEE Trans. Magn., vol. 42, no. 4, pp. 1051-1054, Apr. 2006.

[33] S. Schöps, H. De Gersem, and A. Bartel, "Higher-order cosimulation of field/circuit coupled problems", IEEE Trans. Magn., vol. 48, no. 2, pp. 535-538, Feb. 2012. 
[34] M. J. Gander, "Overlapping schwarz for linear and nonlinear parabolic problems", in 9th International Conference on Domain Decomposition Methods. Ullensvang (Bergen, Norway), 1996, pp. 97104. eprint: https://archive-ouverte unige.ch/unige : 8288.

[35] M. J. Gander, L. Halpern, and F. Nataf, "Optimal convergence for overlapping and non-overlapping schwarz waveform relaxation", in 11th International Conference on Domain Decomposition Methods. Greenwich (Great Britain), 1999, pp. 27-36. eprint: https : / / archive-ouverte . unige . ch / unige : 8286.

[36] M. J. Gander, "Optimized schwarz methods", SIAM Journal on Numerical Analysis, vol. 44, no. 2, pp. 699-731, 2006. eprint: https://doi .org/10.1137/S0036142903425409.

[37] F. Nataf, "Recent developments on optimized Schwarz methods", in Domain Decomposition Methods in Science and Engineering XVI, O. B. Widlund and D. E. Keyes, Eds. Berlin, Heidelberg: Springer Berlin Heidelberg, 2007, pp. 115-125.

[38] M. J. Gander and A. E. Ruehli, "Optimized waveform relaxation methods for RC type circuits", IEEE Trans. Circ. Syst., vol. 51, no. 4, pp. 755-768, Apr. 2004.

[39] M. Al-Khaleel, M. J. Gander, and A. E. Ruehli, "Optimization of transmission conditions in waveform relaxation techniques for RC circuits", SIAM J. Numer. Anal., vol. 52, no. 2, pp. 1076-1101, 2014.

[40] J. d. D. Nshimiyimana, F. Plumier, P. Dular, and C. Geuzaine, "Co-simulation of of finite element and circuit solvers using optimized waveform relaxation", in IEEE International Energy Conference (ENERGYCON) 2016, 2016, pp. 1-6.

[41] J. Nievergelt, "Parallel methods for integrating ordinary differential equations", Comm ACM, vol. 7, pp. 731-733, 1964.

[42] J.-L. Lions, Y. Maday, and G. Turinici, "A parareal in time discretization of PDEs", Comptes Rendus de l'Académie des Sciences - Series I - Mathematics, vol. 332, no. 7, pp. 661-668, 2001.

[43] M. J. Gander and S. Vandewalle, "On the superlinear and linear convergence of the parareal algorithm", in Domain decomposition methods in science and engineering XVI, ser. Lecture Notes in Computational Science and Engineering, vol. 55, Berlin: Springer, 2007, pp. 291-298.

[44] _ _ "Analysis of the parareal time-parallel time-integration method", SIAM Journal on Scientific Computing, vol. 29, no. 2, pp. 556-578, 2007. eprint: https ://doi .org/10.1137/05064607X.

[45] M. J. Gander and E. Hairer, "Nonlinear convergence analysis for the parareal algorithm", in Domain Decomposition Methods in Science and Engineering XVII, U. Langer, M. Discacciati, D. E. Keyes, O. B. Widlund, and W. Zulehner, Eds. Berlin, Heidelberg: Springer Berlin Heidelberg, 2008, pp. 45-56.

[46] R. Lamour, "A shooting method for fully implicit index-2 differential-algebraic equations", Humboldt Universität Berlin, 1994.

[47] _ "A shooting method for fully implicit index-2 differential algebraic equations", SIAM J. Sci. Comput., vol. 18, no. 1, pp. 94-114, Jan. 1997.

[48] T. Cadeau and F. Magoules, "Coupling the parareal algorithm with the waveform relaxation method for the solution of differential algebraic equations", in 10th International Symposium on Distributed Computing and Applications to Business, Engineering and Science, Oct. 2011, pp. 15-19.

[49] S. Schöps, I. Niyonzima, and M. Clemens, "Parallel-in-time simulation of eddy current problems using parareal”, IEEE Trans. Magn., vol. 54, no. 3, pp. 1-4, Mar. 2018. arXiv: 1706.05750.

[50] M. J. Gander, I. Kulchytska-Ruchka, I. Niyonzima, and S. Schöps, "A new parareal algorithm for problems with discontinuous sources", SIAM J. Sci. Comput., vol. 41, no. 2, B375-B395, 2019. arXiv: 1803.05503. 
[51] D. Bast, I. Kulchytska-Ruchka, S. Schöps, and O. Rain, "Accelerated steady-state torque computation for induction machines using parallel-in-time algorithms", IEEE Transactions on Magnetics, vol. 56, no. 2, pp. 1-9, Feb. 2020.

[52] J. Liu and Y.-L. Jiang, "A parareal algorithm based on waveform relaxation", Mathematics and Computers in Simulation, vol. 82, no. 11, pp. 2167-2181, 2012.

[53] M. J. Gander, Y.-L. Jiang, and R.-J. Li, "Parareal schwarz waveform relaxation methods", in Domain Decomposition Methods in Science and Engineering XX, M. Bank Randolph and Holst, O. Widlund, and J. Xu, Eds., Berlin, Heidelberg: Springer Berlin Heidelberg, 2013, pp. 451-458.

[54] M. J. Gander, "50 years of time parallel time integration", in Multiple Shooting and Time Domain Decomposition Methods, T. Carraro, M. Geiger, S. Körkel, and R. Rannacher, Eds., ser. Contributions in Mathematical and Computational Sciences, vol. 9, Springer Berlin Heidelberg, 2015, pp. 69-113.

[55] I. Cortes Garcia, H. De Gersem, and S. Schöps, "A structural analysis of field/circuit coupled problems based on a generalised circuit element", Numer. Algorithm., vol. 83, pp. 373-394, Mar. 2019. arXiv: 1801.07081.

[56] I. Cortes Garcia, S. Schöps, L. Bortot, et al., "Optimized field/circuit coupling for the simulation of quenches in superconducting magnets", IEEE J. Multiscale Multiphys. Comput. Tech., vol. 2, no. 1, pp. 97-104, May 2017. arXiv: 1702.00958.

[57] I. Cortes Garcia, I. Kulchytska-Ruchka, and S. Schöps, "Efficient simulation of field/circuit coupled systems with parallelized waveform relaxation", IEEE Trans. Magn., vol. 56, no. 2, pp. 1-4, 2 Feb. 2020.

[58] I. Cortes Garcia, S. Schöps, H. De Gersem, and S. Baumanns, "Systems of differential algebraic equations in computational electromagnetics", in Applications of Differential-Algebraic Equations: Examples and Benchmarks, A. Campbell Stephenand Ilchmann, V. Mehrmann, and T. Reis, Eds., ser. DifferentialAlgebraic Equations Forum. Heidelberg: Springer, Nov. 2019, pp. 123-169.

[59] J. C. Maxwell, "A dynamical theory of the electromagnetic field", Royal Society Transactions, vol. CLV, pp. 459-512, 1864.

[60] J. D. Jackson, Classical Electrodynamics, 3rd. New York: Wiley \& Sons, 1998.

[61] D. F. Griffiths, Introduction to Electrodynamics. Upper Saddle River, NJ, USA: Prentice-Hall, Sep. 1999.

[62] R. Hiptmair, "Maxwell's equations: Continuous and discrete", in Computational Electromagnetism: Cetraro, Italy 2014, A. Bermúdez de Castro and A. Valli, Eds. Cham: Springer International Publishing, 2015, pp. 1-58.

[63] F. Assous, P. Ciarlet, and S. Labrunie, Mathematical foundations of computational electromagnetism. Springer, 2018.

[64] A. Bossavit, Computational Electromagnetism: Variational Formulations, Complementarity, Edge Elements. San Diego: Academic Press, 1998.

[65] L. Bortot, B. Auchmann, M. Maciejewski, et al., "A 2-d finite-element model for electrothermal transients in accelerator magnets", IEEE Trans. Magn., vol. 54, no. 3, pp. 1-4, Mar. 2018. arXiv: 1710. 01187.

[66] S. Schöps, H. De Gersem, and T. Weiland, "Winding functions in transient magnetoquasistatic fieldcircuit coupled simulations", COMPEL, vol. 32, no. 6, pp. 2063-2083, Sep. 2013.

[67] H. De Gersem, I. Munteanu, and T. Weiland, "Construction of differential material matrices for the orthogonal finite-integration technique with nonlinear materials", IEEE Trans. Magn., vol. 44, no. 6, pp. 710-713, Jun. 2008. 
[68] C. Pechstein and B. Jüttler, "Monotonicity-preserving interproximation of b-h-curves", J. Comput. Appl. Math., vol. 196, no. 1, pp. 45-57, 2006.

[69] C. Pechstein, "Multigrid-Newton-methods for nonlinear-magnetostatic problems", Master's thesis, Universität Linz, Linz, Austria, 2004.

[70] U. Römer, "Numerical approximation of the magnetoquasistatic model with uncertainties and its application to magnet design", Dissertation, Technische Universität Darmstadt, 2015.

[71] Y. Späck-Leigsnering, M. G. Ruppert, E. Gjonaj, H. De Gersem, and V. Hinrichsen, "Thermal instability analysis of station class arresters based on electrothermal finite element simulation", in 20th Proceedings of the International Symposium on High Voltage Engineering (ISH), Budapest, Aug. 2019.

[72] F. Henrotte, B. Meys, H. Hedia, P. Dular, and W. Legros, "Finite element modelling with transformation techniques", IEEE Trans. Magn., vol. 35, no. 3, pp. 1434-1437, May 1999.

[73] H. K. Dirks, "Quasi-stationary fields for microelectronic applications", Electr. Eng., vol. 79, no. 2, pp. 145-155, 1996.

[74] H. A. Haus and J. R. Melcher, Electromagnetic Fields and Energy. Prentice-Hall, 1989.

[75] K. Schmidt, O. Sterz, and R. Hiptmair, "Estimating the eddy-current modeling error", IEEE Trans. Magn., vol. 44, no. 6, pp. 686-689, Jun. 2008.

[76] T. Steinmetz, S. Kurz, and M. Clemens, "Domains of validity of quasistatic and quasistationary field approximations", COMPEL, vol. 30, no. 4, pp. 1237-1247, 2011.

[77] J. Larsson, "Electromagnetics from a quasistatic perspective", Am. J. Phys., vol. 75, no. 3, pp. 230239, 2007.

[78] S. Koch and T. Weiland, "Different types of quasistationary formulations for time domain simulations", Radio Science, vol. 46, no. 5, 2011.

[79] M. Merkel, I. Niyonzima, and S. Schöps, "Paraexp using leapfrog as integrator for high-frequency electromagnetic simulations", Radio Science, vol. 52, no. 12, pp. 1558-1569, Dec. 2017. arXiv: 1705. 08019.

[80] A. Kameari, "Calculation of transient 3D eddy-current using edge elements", IEEE Trans. Magn., vol. 26, no. 5, pp. 466-469, Mar. 1990.

[81] O. Bíró and K. Preis, "On the use of the magnetic vector potential in the finite-element analysis of three-dimensional eddy currents", IEEE Trans. Magn., vol. 25, no. 4, pp. 3145-3159, Jul. 1989.

[82] J. B. Manges and Z. J. Cendes, "Tree-cotree decompositions for first-order complete tangential vector finite elements", Int. J. Numer. Meth. Eng., vol. 40, no. 9, pp. 1667-1685, 1997.

[83] M. Clemens and T. Weiland, "Regularization of eddy-current formulations using discrete grad-div operators", IEEE Trans. Magn., vol. 38, no. 2, pp. 569-572, Mar. 2002.

[84] Q. Chen, W. Schoenmaker, P. Meuris, and N. Wong, "An effective formulation of coupled electromagnetic-TCAD simulation for extremely high frequency onward", IEEE Trans. Circ. Syst., vol. 30, no. 6, pp. 866-876, Jun. 2011.

[85] C. R. I. Emson and C. W. Trowbridge, "Transient 3d eddy currents using modified magnetic vector potentials and magnetic scalar potentials", IEEE Trans. Magn., vol. 24, no. 1, pp. 86-89, Jan. 1988.

[86] C. J. Carpenter, "Comparison of alternative formulations of 3-dimensional magnetic-field and eddycurrent problems at power frequencies", IEE Proceedings B Electric Power Applications, vol. 127, no. 5, p. 332, Sep. 1980. 
[87] J. P. Webb and B. Forghani, "The low-frequency performance of $h-\phi$ and $t-\Omega$ methods using edge elements for 3d eddy current problems", IEEE Trans. Magn., vol. 29, no. 6, pp. 2461-2463, Nov. 1993.

[88] O. Bíró, K. Preis, and K. R. Richter, "Various FEM formulations for the calculation of transient 3d eddy currents in nonlinear media", IEEE Trans. Magn., vol. 31, no. 3, pp. 1307-1312, May 1995.

[89] A. Bossavit, "Differential geometry for the student of numerical methods in electromagnetism", Électricité de France, Tech. Rep., Aug. 1991.

[90] _ _ "On the geometry of electromagnetism. (4): 'Maxwell's house"', JSAEM, vol. 6, no. 4, pp. 318326, 1999.

[91] E. Tonti, "On the formal structure of physical theories", Politecnico di Milano, Milano, Italy, Tech. Rep., 1975.

[92] R. Albanese, E. Coccorese, R. Martone, G. Miano, and G. Rubinacci, "On the numerical solution of the nonlinear three-dimensional eddy current problem", IEEE Trans. Magn., vol. 27, no. 5, pp. 39903995, Sep. 1991.

[93] H. De Gersem and K. Hameyer, "A finite element model for foil winding simulation", IEEE Trans. Magn., vol. 37, no. 5, pp. 3472-3432, Sep. 2001.

[94] S. Schöps, A. Bartel, H. De Gersem, and M. Günther, "DAE-index and convergence analysis of lumped electric circuits refined by 3-d MQS conductor models", in Scientific Computing in Electrical Engineering SCEE 2008, J. Roos and L. R. J. Costa, Eds., ser. Mathematics in Industry, vol. 14, Berlin: Springer, Jun. 2010, pp. 341-350.

[95] H. De Gersem, K. Hameyer, and T. Weiland, "Field-circuit coupled models in electromagnetic simulation", J. Comput. Appl. Math., vol. 168, no. 1-2, pp. 125-133, 2004.

[96] H. De Gersem and T. Weiland, "Field-circuit coupling for time-harmonic models discretized by the finite integration technique", IEEE Trans. Magn., vol. 40, no. 2, pp. 1334-1337, Mar. 2004.

[97] D. N. Dyck and J. P. Webb, "Solenoidal current flows for filamentary conductors", IEEE Trans. Magn., vol. 40, no. 2, pp. 810-813, Mar. 2004.

[98] P. Zhou, Z. Badics, D. Lin, and Z. Cendes, "Nonlinear t- $\Omega$ formulation including motion for multiply connected 3-d problems", IEEE Trans. Magn., vol. 44, no. 6, Jun. 2008.

[99] A. P. Verweij, "Electrodynamics of superconducting cables in accelerator magnets", PhD thesis, Universiteit Twente, Twente, The Netherlands, 1995.

[100] H. De Gersem and T. Weiland, "Finite-element models for superconductive cables with finite interwire resistance", IEEE Trans. Magn., vol. 40, no. 2, pp. 667-670, Mar. 2004.

[101] L. W. Nagel, "SPICE2: A computer program to simulate semiconductor circuits", University of Berkeley, Tech. Rep., 1975.

[102] T. Reis, "Mathematical modeling and analysis of nonlinear time-invariant rlc circuits", in Large-Scale Networks in Engineering and Life Sciences, P. Benner, R. Findeisen, D. Flockerzi, U. Reichl, and K. Sundmacher, Eds. Cham: Springer International Publishing, 2014, pp. 125-198.

[103] C.-W. Ho, A. E. Ruehli, and P. A. Brennan, "The modified nodal approach to network analysis", IEEE Trans. Circ. Syst., vol. 22, no. 6, pp. 504-509, Jun. 1975.

[104] M. Günther, U. Feldmann, and E. J. W. ter Maten, "Modelling and discretization of circuit problems", in Numerical Methods in Electromagnetics, W. H. A. Schilders and E. J. W. ter Maten, Eds., ser. Handbook of Numerical Analysis. Amsterdam, Netherlands: Elsevier BV, 2005, vol. 13, pp. 523-659. 
[105] C. Tischendorf, "Coupled systems of differential algebraic and partial differential equations in circuit and device simulation", Habilitation, Humboldt Universität Berlin, Berlin, Jun. 2003.

[106] P. Monk, Finite Element Methods for Maxwell's Equations. Oxford: Oxford University Press, 2003.

[107] T. Weiland, "A discretization method for the solution of Maxwell's equations for six-component fields", AË̈, vol. 31, pp. 116-120, Mar. 1977.

[108] P. Alotto, A. De Cian, and G. Molinari, "A time-domain 3-D full-Maxwell solver based on the cell method", IEEE Trans. Magn., vol. 42, no. 4, pp. 799-802, Apr. 2006.

[109] A. Bondeson, T. Rylander, and P. Ingelström, Computational Electromagnetics, ser. Texts in Applied Mathematics. Springer, 2005.

[110] K. S. Yee, "Numerical solution of initial boundary value problems involving Maxwell's equations in isotropic media", IEEE Trans. Antenn. Propag., vol. 14, no. 3, pp. 302-307, May 1966.

[111] A. Bossavit, "Computational electromagnetism and geometry: Building a finite-dimensional 'Maxwell's house'. (1): Network equations", JSAEM, vol. 7, no. 2, pp. 150-159, 1999.

[112] T. Weiland, "Time domain electromagnetic field computation with finite difference methods", Int. J. Numer. Model. Electron. Network. Dev. Field, vol. 9, no. 4, pp. 295-319, 1996.

[113] S. Schöps, "Multiscale modeling and multirate time-integration of field/circuit coupled problems", VDI Verlag. Fortschritt-Berichte VDI, Reihe 21, Dissertation, Bergische Universität Wuppertal \& Katholieke Universiteit Leuven, Düsseldorf, Germany, May 2011.

[114] M. Clemens and T. Weiland, "Discrete electromagnetism with the finite integration technique", PIER, vol. 32, pp. 65-87, 2001.

[115] T. Casper, D. Duque, S. Schöps, and H. De Gersem, "Automated netlist generation for 3D electrothermal and electromagnetic field problems", J. Comput. Electron., 2019. arXiv: 1809.08588.

[116] T. Casper, H. De Gersem, R. Gillon, et al., "Electrothermal simulation of bonding wire degradation under uncertain geometries", in Proceedings of the 2016 Design, Automation \& Test in Europe Conference \& Exhibition (DATE), L. Fanucci and J. Teich, Eds., IEEE, Apr. 2016, pp. 1297-1302. arXiv: 1610.04303.

[117] A. Alonso Rodríguez and A. Valli, Eddy Current Approximation of Maxwell Equations, ser. Modeling, Simulation and Applications. Heidelberg: Springer, 2010, vol. 4.

[118] R. Schuhmann and T. Weiland, "Conservation of discrete energy and related laws in the finite integration technique", PIER, vol. 32, pp. 301-316, 2001.

[119] S. Baumanns, "Coupled electromagnetic field/circuit simulation: Modeling and numerical analysis", $\mathrm{PhD}$ thesis, Universität zu Köln, Köln, Sep. 2012.

[120] E. Hairer, S. P. Nørsett, and G. Wanner, Solving Ordinary Differential Equations I: Nonstiff Problems, 2nd ed., ser. Springer Series in Computational Mathematics. Berlin, Germany: Springer, 2000.

[121] — Solving Ordinary Differential Equations II: Stiff and Differential-Algebraic Problems, 2nd ed., ser. Springer Series in Computational Mathematics. Berlin, Germany: Springer, 2002.

[122] R. Mattheij and J. Molenaar, Ordinary Differential Equations in Theory and Practice. Society for Industrial and Applied Mathematics, 2002.

[123] G. G. Dahlquist, "A special stability problem for linear multistep methods", BIT Numerical Mathematics, vol. 3, no. 1, pp. 27-43, Mar. 1963. 
[124] K. E. Brenan, S. L. Campbell, and L. R. Petzold, Numerical Solution of Initial-Value Problems in Differential-Algebraic Equations. Philadelphia, PA, USA: Society for Industrial and Applied Mathematics, 1995.

[125] R. Lamour, R. März, and C. Tischendorf, Differential-Algebraic Equations: A Projector Based Analysis, A. Ilchmann and T. Reis, Eds., ser. Differential-Algebraic Equations Forum. Heidelberg: Springer, 2013.

[126] P. Deuflhard, Newton methods for nonlinear problems: affine invariance and adaptive algorithms. Berlin: Springer, 2004.

[127] T. A. Davis, Direct Methods for Sparse Linear Systems. Society for Industrial and Applied Mathematics, 2006. eprint: https://epubs .siam.org/doi/pdf/10.1137/1.9780898718881.

[128] L. R. Petzold, "Differential/algebraic equations are not ODE's", SIAM J. Sci. Stat. Comput., vol. 3, no. 3, pp. 367-384, Sep. 1982.

[129] D. Estévez Schwarz, "Consistent initialization for index-2 differential algebraic equations and its application to circuit simulation", $\mathrm{PhD}$ thesis, Humboldt-Universität zu Berlin, Mathematisch-Naturwissenschaftliche Fakultät II, 2000.

[130] V. Mehrmann, "Index concepts for differential-algebraic equations", in Encyclopedia of Applied and Computational Mathematics, B. Engquist, Ed. Berlin, Heidelberg: Springer Berlin Heidelberg, 2015, pp. 676-681.

[131] E. Hairer, C. Lubich, and M. Roche, The Numerical Solution of Differential-Algebraic Systems by RungeKutta Methods, ser. Lecture Notes in Mathematics. Berlin: Springer, 1989.

[132] S. Baumanns, M. Selva Soto, and C. Tischendorf, "Consistent initialization for coupled circuit-device simulation", in Scientific Computing in Electrical Engineering SCEE 2008, J. Roos and L. R. J. Costa, Eds., ser. Mathematics in Industry, vol. 14, Berlin: Springer, Jun. 2010, pp. 297-304.

[133] M. Clemens, "Large systems of equations in a discrete electromagnetism: Formulations and numerical algorithms", IEE. Proc. Sci. Meas. Tech., vol. 152, no. 2, pp. 50-72, Mar. 2005.

[134] M. Clemens and T. Weiland, "Transient eddy-current calculation with the FI-method", IEEE Trans. Magn., vol. 35, no. 3, pp. 1163-1166, May 1999.

[135] R. Albanese and G. Rubinacci, "Integral formulation for 3d eddy-current computation using edge elements", IEE. Proc. Sci. Meas. Tech., vol. 135, no. 7, pp. 457-462, Sep. 1988.

[136] I. Munteanu, "Tree-cotree condensation properties", ICS Newsletter (International Compumag Society), vol. 9, pp. 10-14, 2002.

[137] F. Bichet, P. Chombart, and B. Relovsky, "Functional mock-up interface for co-simulation", MODELISAR consortium, Tech. Rep., 2010.

[138] M. J. Gander and L. Halpern, "Optimized schwarz waveform relaxation methods for advection reaction diffusion problems", SIAM J. Numer. Anal., vol. 45, no. 2, pp. 666-697, 2007. eprint: https : //doi.org/10.1137/050642137.

[139] F. Legoll, T. Lelièvre, and G. Samaey, "A micro-macro parareal algorithm: Application to singularly perturbed ordinary differential equations", SIAM J. Sci. Comput., vol. 35, no. 4, A1951-A1986, Aug. 2013.

[140] I. A. Tsukerman, "A stability paradox for time-stepping schemes in coupled field-circuit problems", IEEE Trans. Magn., vol. 31, no. 3, pp. 1857-1860, May 1995. 
[141] T. Dreher and G. Meunier, "3d line current model of coils and external circuits", IEEE Trans. Magn., vol. 31, no. 3, pp. 1853-1856, May 1995.

[142] E. Lange, F. Henrotte, and K. Hameyer, "An efficient field-circuit coupling based on a temporary linearization of FE electrical machine models", IEEE Trans. Magn., vol. 45, no. 3, pp. 1258-1261, 2009.

[143] E. Lelarasmee, "The waveform relaxation method for time domain analysis of large scale integrated circuits: Theory and applications", PhD thesis, EECS Department, University of California, Berkeley, 1982.

[144] M. Maciejewski, I. Cortes Garcia, S. Schöps, et al., "Application of the waveform relaxation technique to the co-simulation of power converter controller and electrical circuit models", in 2017 22nd International Conference on Methods and Models in Automation and Robotics (MMAR), IEEE, Sep. 2017, pp. 837-842. arXiv: 1704.02839.

[145] M. Gander and A. Ruehli, "Optimized waveform relaxation solution of electromagnetic and circuit problems", in 19th Conference on Electrical Performance of Electronic Packaging and Systems (EPEPS), Oct. 2010, pp. 65-68.

[146] M. Al-Khaleel, M. J. Gander, and A. E. Ruehli, "A mathematical analysis of optimized waveform relaxation for a small RC circuit", APNUM, vol. 75, pp. 61-76, 2014.

[147] T. Schierz, M. Arnold, and C. Clauß, "Co-simulation with communication step size control in an FMI compatible master algorithm", in Proceedings of the 9th International Modelica Conference, Munich, Germany, Sep. 2012.

[148] U. Miekkala and O. Nevanlinna, "Convergence of dynamic iteration methods for initial value problems”, SIAM J. Sci. Stat. Comput., vol. 8, pp. 459-482, Jul. 1987.

[149] J. K. White, F. Odeh, A. L. Sangiovanni-Vincentelli, and A. E. Ruehli, "Waveform relaxation: Theory and practice", Transactions of the Society for Computer Simulation, vol. 2, no. 1, pp. 95-133, 1985.

[150] J. Pade and C. Tischendorf, "Waveform relaxation: A convergence criterion for differential-algebraic equations", Numer. Algorithm., Nov. 2018.

[151] J. Pade, I. Cortes Garcia, S. Schöps, and C. Tischendorf, "Waveform relaxation for low frequency coupled field/circuit differential-algebraic models of index 2", in 13th International Conference on Scientific Computing in Electrical Engineering, 2020, to be submitted.

[152] M. J. Gander and T. Vanzan, "Heterogeneous optimized schwarz methods for second order elliptic pdes", SIAM Journal on Scientific Computing, vol. 41, no. 4, A2329-A2354, 2019. eprint: https : //doi.org/10.1137/18M122114X.

[153] L. Bortot, B. Auchmann, I. Cortes Garcia, et al., "A coupled A-H formulation for magneto-thermal transients in high-temperature superconducting magnets", vol. 30, pp. 1-11, 5 2020. eprint: 1909 . 03312.

[154] C. Tischendorf, "Topological index calculation of differential-algebraic equations in circuit simulation", Surv. Math. Ind., vol. 8, no. 3 - 4, pp. 187-199, 1999.

[155] H. Amann and J. Escher, Analysis 2, 2nd ed. Berlin: Springer, 2006.

[156] A. Quarteroni and A. Valli, Domain Decomposition Methods for Partial Differential Equations, ser. Numerical Mathematics and Scientific Computation. Oxford: Oxford University Press, 1999. 
[157] M. J. Gander, I. Kulchytska-Ruchka, and S. Schöps, "Domain decomposition methods in science and engineering XXV", ser. Lecture Notes in Computational Science and Engineering, Springer, 2019, ch. A New Parareal Algorithm for Time-Periodic Problems with Discontinuous Inputs. arXiv: 1810. 12372, Accepted.

[158] Y. Maday, J. Salomon, and G. Turinici, "Monotonic parareal control for quantum systems", SIAM J. Math. Anal., vol. 45, no. 6, pp. 2468-2482, 2007.

[159] D. Estévez Schwarz and R. Lamour, "A new approach for computing consistent initial values and taylor coefficients for daes using projector-based constrained optimization", Numerical Algorithms, vol. 78, no. 2, pp. 355-377, Jun. 2018.

[160] B. Song and Y.-L. Jiang, "Analysis of a new parareal algorithm based on waveform relaxation method for time-periodic problems", Numer. Algorithm., vol. 67, no. 3, pp. 599-622, 2014.

[161] J. Gyselinck, L. Vandevelde, J. Melkebeek, et al., "Calculation of eddy currents and associated losses in electrical steel laminations", IEEE Trans. Magn., vol. 35, no. 3, pp. 1191-1194, May 1999.

[162] D. Meeker, Finite element method magnetics, Version 4.2 (25Feb2018 Build), User's Manual, 2018.

[163] T. Nakamoto, M. Sugano, Q. Xu, et al., "Model magnet development of d1 beam separation dipole for the HL-LHC upgrade", IEEE Trans. Appl. Super., vol. 25, no. 3, pp. 1-5, Jun. 2015.

[164] O. S. Brüning, P. Collier, P. Lebrun, et al., "LHC design report", CERN, Geneva, Report CERN-2004003-V-1, 2004.

[165] COMSOL, COMSOL multiphysics reference manual, 5.2a, 2016.

[166] OrCAD, PSpice user's guide, 17.2, User's Manual, 2016.

[167] L. Bortot, M. Maciejewski, A. M. Prioli Marco Fernandez Navarro, et al., "A consistent simulation of electro-thermal transients in accelerator circuits", IEEE Trans. Appl. Super., vol. 27, no. 4, Jun. 2016.

[168] L. Rossi and M. Sorbi, "Qlasa: A computer code for quench simulation in adiabatic multicoil superconducting windings", Istituto Nazionale di Fisica Nucleare, Milano, Italy, Report INFN/TC-04/13, Jul. 2004.

[169] G. Di Capua and N. Femia, "A novel method to predict the real operation of ferrite inductors with moderate saturation in switching power supply applications", IEEE Trans. Power Electron., vol. 31, no. 3, pp. 2456-2464, Mar. 2016.

[170] J. Roos and L. R. J. Costa, Eds., Scientific Computing in Electrical Engineering SCEE 2008, vol. 14, Mathematics in Industry, Berlin: Springer, Jun. 2010. 


\section{A Appendix}

\section{Abbreviations}

BC boundary condition

BDF backward differentiation formula

BVP boundary value problem

DAE differential algebraic equation

EQS electroquasistatic

ES electrostatic

FEM finite element method

FIT finite integration technique

IC initial condition

IRK implicit Runga Kutta

IVP intial value problem

KCL Kirchhoff's current law

KVL Kirchhoff's voltage law

LTE local truncation error

MNA modified nodal analysis

MQS magnetoquasistatic

MS magnetostatic

ODE ordinary differential equation

PDE partial differential equation

PR Parareal

WR waveform relaxation 


\section{Acknowledgements}

First of all, I would like to thank my supervisor Sebastian Schöps for giving me the opportunity of working in this thesis, helping and guiding me during these years. Also for creating a friendly working atmosphere and making it possible for me to participate in very interesting events during my stay such as the ECMI modelling week.

Secondly, I am also very grateful to Caren Tischendorf, both for the fruitful scientific discussions and collaborations as well as for co-examining this work.

I express all my gratitude to all those other people that collaborated in this work thanks to advices, conversations and help. From Darmstadt side I would like to thank Herbert De Gersem and Iryna Kulchytska-Ruchka for the very interesting scientific discussions, as well as the resulting publications. In addition, a very special thanks to the administrative staff both from the Graduate School as well as TEMF. Moreover, I am very grateful for the nice, important and helpful scientific support and advice from Berlin of Diana Estévez Schwarz, René Lamour, Jonas Pade and Christian Strohm. Last but not least, I thank all our colleagues from the STEAM team at CERN and PSI for this very nice project and interesting collaboration. A special thanks to Michał Maciejewski and Lorenzo Bortot not only for the discussions, but also for providing me with nice simulation results.

I would also like to thank all my Temflepuffers, CEM, Falafel Ladies or whatsoever strange name I can use to denote my colleagues. Their company made the work days, conferences, tea meetings and even weekends both crazy and fun! A special mention to the spanish/quark office for sticking together even in the hardest summer days. Thanks to Melina and Niklas for proofreading and correcting my declension in the German part of this work.

Also thanks to my former professor Susana Serna for motivating me to start a PhD by encouraging my scientific curiosity and sending me to all the interesting workshops I went to during my Bachelor and Master studies.

Finally, I want to thank the extracurricular support; my parents for their cheer and help both during my studies while living together, as well as in my journey abroad and Eric (and Lemmy) for motivating me from the beginning to take a scientific path as well as helping me escape mentally once in a while.

This work is supported by the Excellence Initiative of the German Federal and State Governments and the Graduate School of Computational Engineering at Technical University of Darmstadt and DFG Grant SCHO1562/1-2. 